\title{
Function of the INA complex in assembly of the mitochondrial oxidative phosphorylation system
}

\author{
DISSERTATION \\ for the award of the degree \\ "Doctor rerum naturalium" \\ of the Georg-August-Universität Göttingen \\ within the doctoral program "Molecular Biology" \\ of the Georg-August University School of Science (GAUSS)
}

submitted by

Nataliia Naumenko

from Chernihiv, Ukraine

Göttingen, May 2017 


\section{Members of the Thesis Committee:}

Prof. Dr. Peter Rehling

(Supervisor and first referee)

Prof. Dr. Blanche Schwappach

(Second referee)

Prof. Dr. Reinhard Lührmann
University Medical Center Göttingen

Institute for Cellular Biochemistry

Göttingen, Germany

University Medical Center Göttingen

Institute for Molecular Biology

Göttingen, Germany

Department of Cellular Biochemistry

Max Planck Institute for Biophysical Chemistry

Göttingen, Germany

\section{Additional Members of the Examination Board:}

Prof. Dr. Henning Urlaub

Prof. Dr. Stefan Jakobs

Prof. Dr. Detlef Doenecke
Bioanalytical Mass Spectrometry

Max Planck Institute for Biophysical Chemistry Göttingen, Germany

Structure and Dynamics of Mitochondria Max Planck Institute for Biophysical Chemistry Göttingen, Germany

University Medical Center Göttingen Institute for Molecular Biology

Göttingen, Germany

Date of oral examination: June 19, 2017 


\section{AFFIDAVIT}

Herewith I declare that I prepared my Doctoral Thesis "Function of the INA complex in assembly of the mitochondrial oxidative phosphorylation system" on my own and with no other sources and aids than quoted.

Göttingen, May 2017

Nataliia Naumenko 
Parts of this study are communicated in the following publications:

N. Naumenko, M. Morgenstern, B. Warscheid and P. Rehling. (2017). The INA complex liaises the $\mathrm{F}_{1} \mathrm{~F}_{\mathrm{o}}$-ATP synthase membrane motor modules. Under review.

O. Lytovchenko, N. Naumenko, S. Oeljeklaus, B. Schmidt, K. von der Malsburg, B. Warscheid, M. van der Laan, P. Rehling. (2014). The INA complex facilitates assembly of the peripheral stalk of the mitochondrial $\mathrm{F}_{1} \mathrm{~F}_{\mathrm{o}}$-ATP synthase. EMBO J., 33(15):1624-1638. 
"WORK HARD, PLAY HARD AND SQUEEZE IN TIME TO DO YOUR LAUNDRY."

RON VALE 


\section{Contents}

Table of Contents ix

List of Figures $\quad$ xi

List of Tables $\quad$ xii

Abbreviations xiii

$\begin{array}{ll}\text { Abstract } & 1\end{array}$

1 Introduction $\mathbf{2}$

1.1 Early evolution of eukaryotes . . . . . . . . . . . . . . . 2

1.2 Main features of eukaryotic cells . . . . . . . . . . . . . . . . 3

1.3 Mitochondria . . . . . . . . . . . . . . . 3

1.3.1 Origins of mitochondria . . . . . . . . . . . . 3

1.3.2 Mitochondria-like organelles . . . . . . . . . . . . . . 4

1.3.3 Mitochondrial structure and dynamics . . . . . . . . . . 5

1.3.4 Mitochondrial functions . . . . . . . . . . . . . 6

1.3.5 Import of nuclear-encoded proteins into mitochondria . . . . . 7

1.4 Expression of the mitochondrial genome . . . . . . . . . . . . . . . 9

1.4.1 The mitochondrial genome . . . . . . . . . . . . . . 9 9

1.4.2 Mitochondrial transcription and maturation of transcripts . . 10

1.4.3 Mitochondrial translation . . . . . . . . . . . . . 12

1.4.4 Co-translational membrane protein insertion . . . . . . . . . 14

1.5 Mitochondrial Oxidative Phosphorylation System . . . . . . . . . . 16

1.5.1 Structure and function of OXPHOS system . . . . . . . . 16

1.5.2 Biogenesis of complex III . . . . . . . . . . . . . . . 18

1.5.3 Structure and function of the yeast $\mathrm{F}_{1} \mathrm{~F}_{\mathrm{o}}$-ATP synthase . . . . 22

1.5.4 Mechanism of ATP synthesis by $\mathrm{F}_{1} \mathrm{~F}_{\mathrm{o}}$-ATP synthase . . . . . 24

1.5.5 Biogenesis of mitochondrial-encoded $\mathrm{F}_{1} \mathrm{~F}_{\mathrm{o}}$-ATP synthase subunits ............................. 25

1.5.6 Assembly of the yeast $\mathrm{F}_{1} \mathrm{~F}_{\mathrm{o}}$-ATP synthase . . . . . . . . 27 
1.5.7 Role of the INA complex in $\mathrm{F}_{1} \mathrm{~F}_{\mathrm{o}}$-ATP synthase assembly . . . 29

1.6 Aim of this study . . . . . . . . . . . . . . . . . . . . . 31

\section{Results}

2.1 Deletion of INA complex subunits affects biogenesis of mitochondrialencoded $\mathrm{F}_{1} \mathrm{~F}_{\mathrm{o}}$-ATP synthase components . . . . . . . . . . . . . . 32

2.1.1 Translation of Atp6 and Atp8 is upregulated in ina mutants, whereas Atp9 is destabilized . . . . . . . . . . . . 32

2.1.2 INA complex does not associate with mitochondrial ribosomes 34

2.1.3 Atp6 processing is impaired in ina22 $\ldots . . . . . . . .35$

2.1.4 Atp23 overexpression rescues the Atp6 processing defect in ina22 . . . . . . . . . . . . . . . . 37

2.1.5 Atp23 overexpression partially rescues $\mathrm{F}_{1} \mathrm{~F}_{\mathrm{o}}$-ATP synthase assembly defects in ina22 $\ldots \ldots \ldots . \ldots . \ldots . \ldots 37$

2.2 The C-terminus of Ina22 is highly unstable . . . . . . . . . . . . . . . 41

2.3 INAC interacts with the Atp6/Atp8 biogenesis machinery . . . . . . . 43

2.3.1 INAC associates with Atp23 . . . . . . . . . . . . 43

2.3.2 INAC interacts with Atp10 . . . . . . . . . . . . . 44

2.4 Stalling of $\mathrm{F}_{1} \mathrm{~F}_{\mathrm{o}}$-ATP synthase assembly in ina mutants . . . . . . . . 48

2.4.1 Atp10 accumulates with Atp23 upon INA22 deletion . . . . . 48

2.4.2 Atp23 association with the IMM is increased in ina22 $\Delta$. . . . 49

2.4.3 The Atp6/Atp8 module accumulates upon INA22 deletion . . 49

2.4.4 $\quad \mathrm{F}_{1}$ and the peripheral stalk accumulate with Atp10 in ina22 $\Delta 52$

2.4.5 $\quad \mathrm{F}_{1}$ and the peripheral stalk assemble with Atp6/Atp8 module. 53

2.4.6 $\quad \mathrm{F}_{1} \mathrm{~F}_{\mathrm{o}}$-ATP synthase assembly is stalled in ina22 $\Delta$ prior to the last step . . . . . . . . . . . . . . . . . . 5 5

2.5 Ina22 associates with two major $\mathrm{F}_{1} \mathrm{~F}_{\mathrm{o}}$-ATP synthase assembly intermediates to link them together . . . . . . . . . . . . . . . 57

2.5.1 Ina22 interacts with newly translated mitochondrial-encoded proteins . . . . . . . . . . . . . . . 57

2.5.2 Ina22 interaction with $\mathrm{F}_{1} \mathrm{~F}_{\mathrm{o}}$-ATP synthase is Ina17-independent 57

2.5.3 Ina22 interacts with the Atp9 ring and the Atp6/Atp8 module 60

2.5.4 Ina22 can be cross-linked to the Atp9 ring and the peripheral stalk . . . . . . . . . . . . . . . . 6 61

2.6 INAC function in assembly of cytochrome $b c_{1}$ complex . . . . . . . . 62

2.6.1 The INA complex interacts with Cbp proteins . . . . . . . . 62

2.6.2 INAC interacts with Cbps in a cytochrome $b$ - dependent manner ....................... 63

2.6.3 A C-terminal protein A tag does not alter the function of Cbp proteins ....................... 65 
2.6.4 INAC interacts with Cbps in a discrete protein complex . . . . 65

2.6.5 Newly translated cytochrome $b$ is present in INAC*CBP complex ............................ 67

2.6.6 The INAC*CBP complex is destabilized upon complex III assembly stalling . . . . . . . . . . . . . . . . 69

2.6.7 INAC does not associate with known cytochrome $b$ translational activators . . . . . . . . . . . . . . 70

2.6.8 Proof of INAC* ${ }^{*} \mathrm{CBP}$ interaction . . . . . . . . . . . . . 72

3 Discussion $\quad \mathbf{7 5}$

3.1 Altered expression of mitochondrial-encoded $\mathrm{F}_{1} \mathrm{~F}_{\mathrm{o}}$-ATP synthase components in ina mutants . . . . . . . . . . . . . . . . . 76

3.2 Atp6 processing defect as a marker for impaired $F_{o}$ biogenesis . . . . 77

3.3 INAC association with the Atp6/Atp8 biogenesis machinery . . . . . 79

$3.4 \quad \mathrm{~F}_{1} \mathrm{~F}_{\mathrm{o}}$-ATP synthase assembly is stalled before its final step in ina22 $\Delta \quad 80$

3.5 INAC mediates formation of the membrane proton channel in the last step of complex V formation . . . . . . . . . . . . . . . . 82

3.6 A novel model of $\mathrm{F}_{1} \mathrm{~F}_{\mathrm{o}}$-ATP synthase assembly . . . . . . . . . . . . . 84

3.7 Evolutionary conservation of the INA complex and the $\mathrm{F}_{1} \mathrm{~F}_{\mathrm{o}}$-ATP synthase assembly pathway. . . . . . . . . . . . . 86

3.8 C-terminal processing of Ina22 . . . . . . . . . . . . . . . . . 88

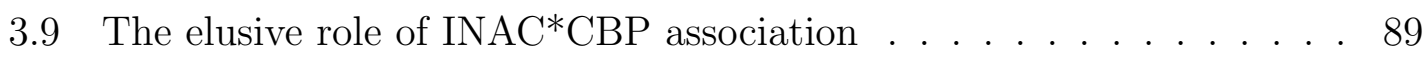

4 Conclusions and Perspectives $\quad 91$

5 Materials and Methods $\quad 93$

5.1 Materials .......................... 93

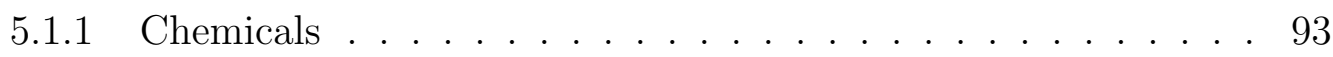

5.1 .2 Buffers and solutions . . . . . . . . . . . . . . . 95

5.1 .3 Kits and disposables . . . . . . . . . . . . . . . . . 97

5.1 .4 Equipment. . . . . . . . . . . . . . . 97

5.1 .5 Software . . . . . . . . . . . . . . . . . 99

5.1 .6 Antibodies ....................... 99

5.1 .7 Yeast strains used in this study . . . . . . . . . . . . . . 100

5.1 .8 Oligonucleotides used in this study . . . . . . . . . . . 102

5.1 .9 Plasmids used in this study . . . . . . . . . . . . . . . 102

5.1 .10 E. coli strains used in this study . . . . . . . . . . . . . 102

$5.2 \quad$ E. coli methods . . . . . . . . . . . . . . . . . . . . 106

$5.2 .1 \quad$ E. coli growth . . . . . . . . . . . . . . . 106

5.2 .2 E. coli transformation . . . . . . . . . . . . . 106

5.3 S. cerevisiae cultivation and preparation of mitochondria . . . . . 106 
5.3.1 Cultivation of S. cerevisiae . . . . . . . . . 106

5.3 .2 Growth test . . . . . . . . . . . . . . . . . . . 107

5.3 .3 Yeast transformation . . . . . . . . . . . . . 107

5.3.4 Preparation of mitochondria . . . . . . . . . . . . . 107

5.3.5 S. cerevisiae whole-cell extract preparation . . . . . . . . . 108

5.4 Methods for analysis of proteins and protein complexes . . . . . . . . 108

5.4.1 Determination of protein concentration . . . . . . . . . 108

5.4 .2 SDS-PAGE . . . . . . . . . . . . . . . . 109

5.4 .3 Urea SDS-PAGE . . . . . . . . . . . . . . . . . . 109

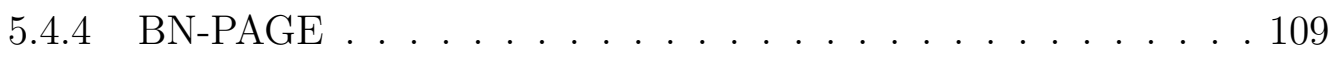

5.4.5 Western blotting and immunodecoration . . . . . . . . . . 110

5.4.6 Coomassie Brilliant Blue staining . . . . . . . . . . . . . . . 110

5.4.7 Colloidal Coomassie staining . . . . . . . . . . . . . . 110

5.4 .8 IgG affinity chromatography . . . . . . . . . . . . . . . . 111

5.4 .9 Protein immunoprecipitation . . . . . . . . . . . . . . 111

5.4.10 FLAG-affinity purification . . . . . . . . . . . . . . . 112

5.4 .11 In vivo labeling of mitochondrial-encoded proteins . . . . . . . 112

5.4 .12 TCA protein precipitation . . . . . . . . . . . 113

5.4.13 Digital autoradiography . . . . . . . . . . . . . . . 113

5.5 Molecular biology techniques . . . . . . . . . . . . . . . . 113

5.5.1 Preparation of genomic DNA from S. cerevisiae . . . . . . . 113

5.5.2 Plasmid isolation from E. coli . . . . . . . . . . . . 114

5.5.3 Polymerase chain reaction (PCR) . . . . . . . . . . . . . 114

5.5.4 Agarose gel electrophoresis . . . . . . . . . . . . . . . . . . . 114

5.5.5 Purification of PCR products . . . . . . . . . . . . . 115

5.5.6 Molecular cloning . . . . . . . . . . . . . . . . 115

5.5.7 In vitro mutagenesis . . . . . . . . . . . . . . . . . . . 115

5.5.8 In vitro mRNA synthesis . . . . . . . . . . . . . . . . . . . . 115

5.5.9 Synthesis of radiolabeled protein precursors . . . . . . . . 116

5.5.10 Determination of nucleic acid concentration . . . . . . . . 116

5.6 Assays with purified mitochondria . . . . . . . . . . . . 116

5.6.1 Solubilization of mitochondrial membranes . . . . . . . . . . 116

5.6.2 Import of radiolabeled protein precursors into mitochondria . 116

5.6.3 In organello protein labeling with $\left[{ }^{35} \mathrm{~S}\right]$ Met . . . . . . . . . . . 117

5.6.4 Proteinase K protection assay . . . . . . . . . . . . . . 117

5.6.5 Carbonate extraction of proteins . . . . . . . . . . 117

5.6.6 Determination of in-gel activity of $\mathrm{F}_{1} \mathrm{~F}_{\mathrm{o}}$-ATP synthase . . . . 118

5.6.7 ATPase activity measurement with and without oligomycin . . 118

5.6.8 Fractionation of mitochondrial lysate through a sucrose cushion 118

5.6.9 Chemical protein cross-linking in mitochondria . . . . . . . . 119 
5.6.10 Protein assembly into mitochondrial protein complexes . . . . 119

5.7 Miscellaneous . . . . . . . . . . . . . . . . . . . . . . . . . . 119

Bibliography

Acknowledgements 


\section{List of Figures}

1.1 A schematic representation of the mitochondrion . . . . . . . . . 5

1.2 Organisation of the S. cerevisiae mitochondrial genome. . . . . . . . . 10

1.3 Mitochondrial-encoded membrane proteins of S. cerevisiae. . . . . . . 14

1.4 Mitochondrial OXPHOS system. . . . . . . . . . . . . . . . . 17

1.5 A schematic representation of cytochrome $b$ assembly. . . . . . . . . . 21

1.6 Structure of the S. cerevisiae $\mathrm{F}_{1} \mathrm{~F}_{\mathrm{o}}$-ATP synthase complex. . . . . . . 24

1.7 Schematic representation of early Atp6/Atp8 biogenesis. . . . . . . . 27

1.8 Model of $\mathrm{F}_{1} \mathrm{~F}_{\mathrm{o}}$-ATP synthase assembly. . . . . . . . . . . . . . . 28

2.1 Atp6 translation is upregulated in ina22 $\Delta$ and ina17 $4 . . . . . . .33$

2.2 Atp8 and Atp9 levels are changed in ina mutants. . . . . . . . . . . . 34

2.3 INA complex does not associate with mitochondrial ribosomes. . . . . 35

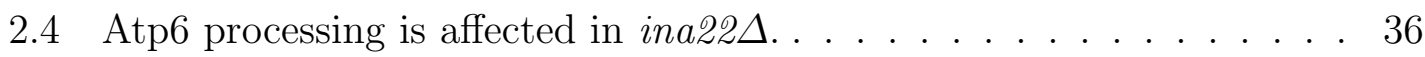

2.5 Atp23 overexpression rescues the Atp6 processing defect in ina224. . 38

2.6 Atp23 overexpression partially rescues $\mathrm{F}_{1} \mathrm{~F}_{\mathrm{o}}$-ATP synthase assembly

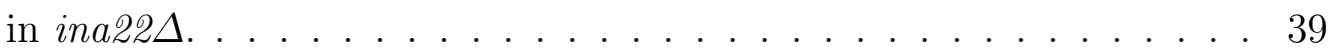

2.7 Atp23 ${ }^{\mathrm{H} 128 \mathrm{~A}}$ overexpression does not restore the $\mathrm{F}_{1} \mathrm{~F}_{\mathrm{o}}$-ATP synthase

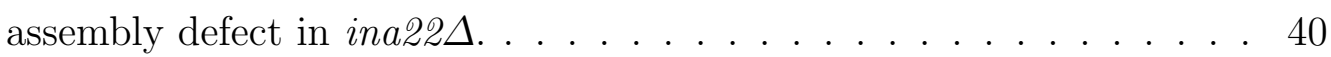

2.8 Ina22 undergoes C-terminal processing. . . . . . . . . . . . . . . . . 42

2.9 INAC interacts with Atp23. . . . . . . . . . . . . . . . . 44

2.10 Upregulation of mitochondrial translation increases INAC-Atp23 association. . . . . . . . . . . . . . . . . . 45

2.11 C-terminal tagging does not affect Atp10 function . . . . . . . . . . . 46

2.12 INAC associates with Atp10. . . . . . . . . . . . . . . . 47

2.13 Atp23 accumulates with Atp10 upon INA22 deletion. . . . . . . . . . 48

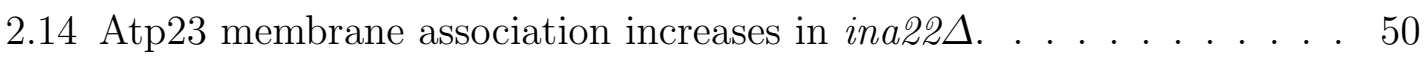

2.15 Atp10 interaction with Atp6 and Atp8 is increased in ina22 $\Delta$. . . . . 51

$2.16 \mathrm{~F}_{1}$ and the peripheral stalk accumulate with Atp10 in ina22. . . . . 52

$2.17 \mathrm{~F}_{1}$ and the peripheral stalk assemble on the Atp6/Atp8 module. . . . 54

$2.18 \mathrm{~F}_{1}$ and Atp23 associate with the Atp6/Atp8 module. . . . . . . . . . 55

$2.19 \mathrm{~F}_{1} \mathrm{~F}_{\mathrm{o}}$-ATP synthase assembly is stalled before the final step. . . . . . 56 
2.20 Ina22 interacts with newly translated mitochondrial-encoded proteins. 58

2.21 Ina22 interaction with $\mathrm{F}_{1} \mathrm{~F}_{\mathrm{o}}$-ATP synthase is Ina17-independent. . . . 59

2.22 Ina22 interacts with the Atp9 ring and the Atp6/Atp8 module. . . . . 60

2.23 Ina22 can be crosslinked to Atp9 ring and Atp4. . . . . . . . . . . . . 62

2.24 The INA complex interacts with Cbp proteins. . . . . . . . . . . . . 63

2.25 The Ina22 interaction with Cbps is cytb-dependent. . . . . . . . . . . 64

2.26 C-terminal protein A tag does not alter the function of Cbp proteins. 66

2.27 INAC-Cbps containing protein complex can be natively isolated. . . . 67

2.28 Newly translated cytochrome $b$ is present in the INAC ${ }^{*} \mathrm{CBP}$ complex. 68

2.29 The $\mathrm{INAC}^{*} \mathrm{CBP}$ complex is unstable upon $C B P 6$ deletion. . . . . . . 70

2.30 The INAC* CBP complex is destabilized upon complex III assembly stalling. . . . . . . . . . . . . . . . . 71

2.31 INAC does not associate with the cytochrome $b$ translational activators Cbs1 and Cbs2. . . . . . . . . . . . . . . . 72

2.32 INAC interaction with Cbps is not a post-lysis artifact. . . . . . . 73

3.1 A novel model for $\mathrm{F}_{1} \mathrm{~F}_{\mathrm{o}}$-ATP synthase assembly. . . . . . . . . . . . 85 


\section{List of Tables}

1.1 Factors involved in the expression of the $S$. cerevisiae genome . . . . 11

1.2 Subunits of the S. cerevisiae ATP synthase . . . . . . . . . . 23

5.1 List of chemicals . . . . . . . . . . . . . . . . . . . 93

5.2 Buffers and solutions . . . . . . . . . . . . . . . . . . 95

5.3 Kits and disposables . . . . . . . . . . . . . . . . . . . 97

5.4 Equipment . . . . . . . . . . . . . . . . . . . . . . . . 98

5.5 Software used in this study . . . . . . . . . . . . . . . . . . 99

5.6 Antibodies used in this study . . . . . . . . . . . . . . . . . . 99

5.7 Yeast strains . . . . . . . . . . . . . . . . . . . . . . 100

5.8 Plasmids . . . . . . . . . . . . . . . . . . . . . . 102

5.9 Bacterial strains . . . . . . . . . . . . . . . . . . . . . . . 102

5.10 Oligonucleotides . . . . . . . . . . . . . . . . . 103 


\section{Abbreviations}

ATP
BN-PAGE
BSA
COX
Cyt $b$
DNA
DDM
dNTP
DTT
EDTA
FeS
FMN
HEPES
IgG
IMM
IMS
INAC
kDa
MOPS
mRNA
NADH
OMM
OXPHOS
PAGE
PAM
P
PCR
PMSF
PVDF
RNA
rpm
S. cerevisiae
SAM
SEM bufer
SILAC
SDS
SDS-PAGE
TA
TAE
TCA
TIM22
TIM23
TOM
WT
w/v
$\psi$

adenosine triphosphate

blue native polyacrylamide gel electrophoresis

bovine serum albumine

cytochrome c oxidase

cytochrome $b$

deoxyribonucleic acid

dodecylmaltoside

2'-deoxynucleoside-5'-triphosphate

dithiothreitol

ethylenediaminetetraacetic acid

iron-sulfur

flavin mononucleotide

4-(2-hydroxyethyl)-1-piperazineethanesulfonic acid immunoglobulin $\mathrm{G}$

inner mitochondrial membrane

intermembrane space

Inner Membrane Assembly Complex

kilodalton

morpholinopropanesulfonic acid

messenger RNA

nicotinamide adenine dinucleotide (reduced)

outer mitochondrial membrane

oxidative phosphorylation

polyacrylamide gel electrophoresis

presequence translocase associated motor

inorganic phosphate

polymerase chain reaction

phenylmethylsulfonylfluoride

polyvinylidene fluoride

ribonucleic acid

rotations per minute

Saccharomyces cerevisiae

sorting and assembly machinery of the outer membrane sucrose/EDTA/MOPS buffer

Stable isotope labeling with amino acids in cell culture sodium dodecyl sulfate

sodium dodecyl sulfate-polyacrylamide gel elctrophoresis translation activation

Tris/Acetate/EDTA buffer

trichloroacetic acid

carrier translocase of the inner mitochondrial membrane

presequence translocase of the inner mitochondrial membrane

translocase of the outer mitochondrial membrane

wild type

weight/volume

membrane potential 


\section{Abstract}

The majority of cellular ATP is produced by the mitochondrial $\mathrm{F}_{1} \mathrm{~F}_{\mathrm{o}}$-ATP synthase, also called complex $\mathrm{V}$, which consists of nuclear- and mitochondrial-encoded subunits. In order to form a functional enzyme, its proteins must be expressed at appropriate levels, delivered to the correct destination within mitochondria and then properly assembled. Proteins called assembly factors assist in the assembly process and tightly regulate it on various levels.

The $\mathrm{F}_{1} \mathrm{~F}_{\mathrm{o}}$-ATP synthase is thought to assemble in a modular way, involving the formation of distinct assembly intermediates that associate with each other in a defined order. Importantly, formation of the proton-conducting membrane channel is the last step of complex $\mathrm{V}$ biogenesis. The channel-forming Atp6 and Atp9 subunits are retained within two different assembly intermediates and their association generates a mature $\mathrm{F}_{1} \mathrm{~F}_{\mathrm{o}}$-ATP synthase. How this last step of complex $\mathrm{V}$ biogenesis is regulated in order to prevent premature proton-channel formation and thus membrane potential dissipation is currently poorly understood.

Recently, the Inner Membrane Assembly Complex (INAC) was identified that participates in the assembly of yeast $\mathrm{F}_{1} \mathrm{~F}_{\mathrm{o}}$-ATP synthase. Its two members, Ina22 and Ina17, were shown to physically associate with nuclear-encoded $\mathrm{F}_{1} \mathrm{~F}_{\mathrm{o}}$-ATP synthase subunits and to facilitate assembly of the peripheral stalk of complex V. In this study, we revise the current knowledge of $\mathrm{F}_{1} \mathrm{~F}_{\mathrm{o}}$-ATP synthase biogenesis and describe a novel regulatory role of INAC. We found that INAC interacts not only with nuclear-encoded, but also with mitochondrial-encoded structural subunits, as well as other assembly factors of the $\mathrm{F}_{1} \mathrm{~F}_{\mathrm{o}}$-ATP synthase, within two distinct assembly intermediates. One of these intermediates contains Atp6 and the other one contains the Atp9 ring, that together form a proton-conducting channel. We found that, in contrast to what was known before, all nuclear-encoded subunits associate with the Atp6-containing assembly intermediate prior to their interaction with the Atp9 ring. Therefore, a novel $\mathrm{F}_{1} \mathrm{~F}_{\mathrm{o}}$-ATP synthase assembly intermediate was characterized in this study.

Our extensive analyses revealed that the INA complex is involved in the most critical step of complex $\mathrm{V}$ biogenesis, namely formation of the proton-conducting membrane channel. Therefore, INAC ensures the tight coupling of proton movement to ATP production. Moreover, our data provides novel insights into the composition of assembly intermediates of complex $\mathrm{V}$ and extends our understanding of its assembly pathway. 


\section{Introduction}

\subsection{Early evolution of eukaryotes}

Oxygen is one of the life-defining elements on Earth. Changes in the concentration of this element can be tracked throughout Earth's history and correspond to the thrive and decline of various life forms on our planet. Moreover, increased concentrations of atmospheric oxygen enabled the development of efficient energy production systems, such as oxidative phosphorylation, and a rapid expansion of eukaryotic forms of life and multicellular organisms.

Geologically, Earth was formed around 4.5 billion years ago and remained uninhabited for almost one billion years (Dalrymple, 2001). The atmosphere of our planet at that time contained very little free oxygen, if at all. It was saturated with gases like $\mathrm{CO}_{2}, \mathrm{~N}_{2}, \mathrm{H}_{2} \mathrm{~S}$, CO and was highly reducing (Kasting, 1993; Dismukes et al., 2001; Kump, 2008). It was shown already at the beginning of the last century that, when given an energy source, organic molecules can be spontaneously formed from a gas mixture that resembles the early atmosphere on Earth (Miller, 1953). Whether this complex chemistry has indeed created early life on our planet is still a matter of debate. However, it is known that the first bacterial forms of life appeared around 3.7 billion years ago and produced their energy by anoxygenic photosynthesis, using hydrogen and sulphur as electron donors (Olson, 2006).

It is generally accepted that cyanobacteria were the first organisms to produce oxygen and, therefore, their appearance on Earth around 2.5 billion years ago predated aerobic respiration (Planavsky et al., 2014). The Great Oxygenation Event, caused by a rapid increase in atmospheric oxygen concentration by photosynthetic cyanobacteria, induced the first known massive extinction on Earth that wiped out the majority of all existing obligate anaerobic species.

The increase in atmospheric oxygen concentration triggered the development of a principally novel aerobic metabolism and, consequently, the eukaryotic expansion. In the presence of oxygen, sugars became a significantly more efficient energy source, giving up to 18 times more ATP compared to anaerobic metabolism (Dismukes et al., 2001). Such an increase in energy supply made it possible for complex biochemical processes to evolve within the cell, eventually creating the biochemical complexity of eukaryotes. 


\subsection{Main features of eukaryotic cells}

Eukaryotic cells differ significantly from prokaryotes. First of all, eukaryotes enclose their genetic information within a separate compartment, the nucleus, and therefore strictly separate nuclear transcription from cytoplasmic translation. Second, they are almost ten times bigger in size and contain intracellular membraneenclosed compartments called organelles (Palade, 1964). Each organelle in the cell is surrounded by at least one membrane, built up from two layers of bi-polar phospholipids. Membranes create a hydrophobic barrier in the hydrophilic cytoplasm that do not allow molecules to freely diffuse within the cell. This, in turn, allows each organelle to create and to maintain a unique biochemical environment, including $\mathrm{pH}$, ion concentration and a specialized proteome. Therefore, eukaryotic cells evolved and sustained an enormous complexity. In fact, biochemically incompatible anabolic and catabolic reactions can be differentially regulated due to their spatial separation.

Despite all the advantages, eukaryotes faced new problems associated with increased energy demands for maintaining complex processes and regulatory networks. Interestingly, the most energy-requiring processes in the cell are associated with the quality control of transcription, mRNA splicing and maturation, protein synthesis, protein targeting and homeostasis (Liberek et al., 2008; Amma et al., 2008). Therefore, eukaryotic cells rely much more on aerobic energy production via oxidative phosphorylation than prokaryotes. Simply because aerobic oxidation of substrates is much more efficient than anaerobic, the development of the oxidationphosphorylation (OXPHOS) system allowed eukaryotes to thrive and multicellular organisms to evolve. Interestingly, all eukaryotes contain their OXPHOS machinery within a unique organelle, the mitochondrion.

\section{$1.3 \quad$ Mitochondria}

\subsubsection{Origins of mitochondria}

Mitochondria appeared as a result of a single endosymbiotic event between two prokaryotic organisms approximately 1.5 billion years ago. According to the most accepted hypothesis, the host cell, an obligate anaerobe, engulfed an endosymbiotic cell by active phagocytosis. The endosymbiont was an aerobe that used metabolites, produced by the host cell, and detoxified oxygen. Complete sequencing of numerous mitochondrial and prokaryotic genomes showed that mitochondria most likely originate from an $\alpha$-proteobacterial ancestor (Lang et al., 1999; Andersson et al., 2003). Interestingly, the genome of the bacterium Rickettsia prowazekii is the most "mitochondria-like" and resembles tremendously the most "bacteria-like" mitochondrial genome of Reclinomonas americana (Andersson et al., 1998). An 
extensive sequencing of various mitochondrial genomes showed that they are exclusively dedicated to mitochondrial translation, electron transport chain function and the production of ATP (Gray et al., 1999; Lang et al., 1999). Moreover, sequencing has revealed that most mitochondrial genomes underwent a "reductive evolution", resulting in smaller genomes encoding less proteins in higher eukaryotes, compared to the large ancestral-like mitochondrial genomes of protists (Andersson and Kurland, 1998). The reduction of the genome capacity happened mostly due to the transfer of mitochondrial-encoded genes to the nucleus, which is still an ongoing process in many flowering plants (Martin and Herrmann, 1998). But, in some cases, the function of mitochondrial gene products was taken over by unrelated nuclear-encoded proteins. One such example is the substitution of a bacteria derived multisubunit RNA-polymerase to a single-subunit bacteriophage T3/T7-like polymerase in mitochondria of almost all eukaryotes (Gray and Lang, 1998). In some cases, however, mitochondrial genes were simply lost and never functionally replaced. This happened, for example, with the $S$. cerevisiae respiratory chain complex I, which is no longer present in this species (Kurland and Andersson, 2000).

\subsubsection{Mitochondria-like organelles}

"Mitochondria", in a broader sense, is a family of related organelles present in various species of the eukaryotic phylum. They can be divided into four major groups based on whether they produce ATP and on their mechanism for ATP production.

Mitochondria. Mitochondria, such as those present in mammalian cells, use molecular oxygen $\left(\mathrm{O}_{2}\right)$ as a final electron acceptor to produce up to $36 \mathrm{~mol}$ of ATP per mol of glucose. Water and carbon dioxide are their by-products. Mitochondria contain their own DNA, translation machinery and an electron-transport chain. These organelles are typical for higher eukaryotes, plants and many protists.

Anaerobic mitochondria. Many invertebrate species adapted to life in anaerobic conditions. Their mitochondria do not use $\mathrm{O}_{2}$ as a final electron acceptor and they therefore excrete end products like carbon dioxide, acetate, propionate and succinate. Although these organelles retained their electron-transport chain, they are less efficient than aerobic mitochondria and produce only 5 mol of ATP per mol of glucose (Mentel and Martin, 2008).

Hydrogenosomes. Hydrogenosomes were discovered in 1973 in the unicellular eukaryotes, Trychomonads, and since then in many more unrelated species (Müller and Fox, 1984; Müller, 1993). These organelles produce ATP by a simple fermentation of pyruvate and produce molecular hydrogen as a final endproduct. Aside for few exceptions, hydrogenosomes do not have their own genome or electron-transport chain (Boxma et al., 2005). Two different hypotheses exist on their origin. According to the first, hydrogenosomes evolved from aerobic mitochondria after the acquisition of anaerobic enzymes (Embley et al., 1997). The second hypothesis claims that 
mitochondria and hydrogenosomes evolved simultaneously from a common ancestor and specialized later to aerobic and anaerobic conditions, respectively (Martin et al., 2001).

Mitosomes. This last group of mitochondria-like organelles is not involved in ATP synthesis, as all necessary enzymes were transferred to the cytoplasm. Mitosomes have neither a genome, nor the respiratory chain and protein translation machinery (Mai et al., 1999; Tovar et al., 1999; von der Giezen and Tovar, 2005). The only function which they fulfill is the production of FeS clusters as enzymatic co-factors, a process that, with one known, exception can not be done anywhere else in the eukaryotic cell (Müller, 1993; Karnkowska et al., 2016).

\subsubsection{Mitochondrial structure and dynamics}

As a result of their endosymbiotic origin, mitochondria are surrounded by two membranes. The outer mitochondrial membrane (OMM) originates from the plasma membrane of the host cell, whereas the inner mitochondrial membrane (IMM) once belonged to the engulfed $\alpha$-proteobacterium (Fig. 1.1). Therefore, two soluble compartments are separated in the mitochondria - the matrix and the inter membrane space (IMS).

The OMM does not create a barrier for small molecules due to the presence of porins. Therefore, ion concentrations in the cytoplasm and the IMS do not differ significantly (Benz, 1994). On the other hand, the IMM is highly impermeable to small molecules allowing mitochondria to establish an electrochemical gradient across the IMM, which drives protein import into the organelle (discussed in 1.3.5) and ATP production. The IMM has a much larger surface area compared to the

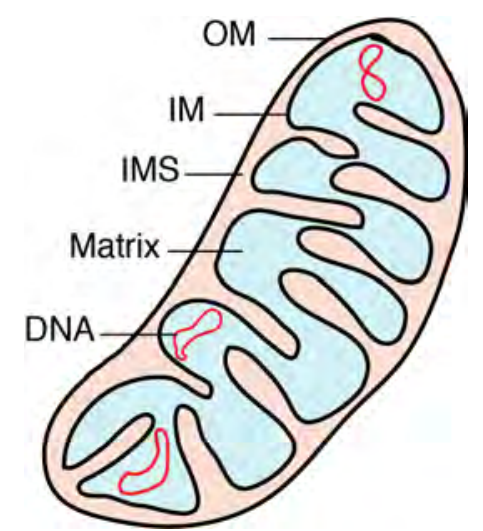

Figure 1.1 A schematic representation of the mitochondrion. The mitochondrion is an organelle with two membranes and two soluble compartments. OMM, outer mitochondrial membrane, IMM, inner mitochondrial membrane, IMS, inter membrane space.

OMM and forms invaginations towards the matrix, called cristae. The IMM can be divided into structurally and functionally distinct domains. The inner boundary membrane is located close to the OMM and houses protein import machinery, as here 
the translocases of the inner and the outer membranes come into contact. Cristae junctions are sites where the inner boundary membrane bends to form cristae. A highly specialized protein machinery has evolved to maintain cristae junctions the MICOS complex (Mitochondrial Contact site and Cristae Organization System) (van der Laan et al., 2012). Moreover, cristae are additionally stabilized by dimers of $\mathrm{F}_{1} \mathrm{~F}_{\mathrm{o}}$-ATP synthase which, together with MICOS, maintain the unique ultastructure of the IMM (Arnold et al., 1998; Paumard et al., 2002).

Mitochondria are very dynamic organelles that form an interconnected network in the cell. The size of this network is controlled by the opposing processes of fusion and fission. In human, fission is performed by Dynamin-related protein 1 (DRP1) and results in fragmentation of the mitochondrial network that facilitates autophagic removal of damaged mitochondria (Taguchi et al., 2007). Fusion, on the other hand, is performed by Mitofusin 1 and 2 (MFN1, MFN2), two GTP-binding proteins of the OMM (Chen et al., 2003) and Optic Atrophy 1 (OPA1) (Ishihara et al., 2006). Balance between fusion and fission events is necessary for proper mitochondrial function and quality control. The mitochondrial network responds greatly to external signals, such as nutrient availability, stress, diseases, and varies significantly between organs and tissues (Wai and Langer, 2016). Therefore, the regulation of mitochondrial dynamics is key to efficient mitochondrial adaptation and is one of the main topics of current mitochondrial research.

\subsubsection{Mitochondrial functions}

Mitochondria fulfill various functions in the cell, including energy production, fatty acid and lipid biosynthesis, biosynthesis of heme and iron-sulphur (FeS) clusters (Lill and Mühlenhoff, 2008, Tovar et al., 2003), $\mathrm{Ca}^{2+}$-signaling (Clapham, 2007), programmed cell death (Green and Reed, 1998) and ROS (reactive oxygen species) signaling (Shadel and Horvath, 2015). They have been implicated in aging and cancer and their disfunction is associated with many severe disorders. Recent discoveries have shown that mitochondria maintain physical contacts with other cellular organelles, like the ER (Mishra and Chan, 2017). Moreover, they are important for intracellular communication and maintenance of cytoplasmic protein homeostasis (Ruan et al., 2017). Surprisingly, mitochondria have recently been suggested to be involved in the biogenesis of peroxisomes (Sugiura et al., 2017).

Though it is generally assumed that the main function of mitochondria is ATP synthesis, there are organisms for which this process is dispensable. Yeast $S$. cerevisiae can switch between aerobic and anaerobic metabolism, implying that energy production by mitochondria is not essential. However, yeast can not survive without mitochondria, meaning that the organelle is essential per se. The understanding of this phenomena came when mitosomes were discovered. Mitosomes do not have an electron transport chain and do not produce ATP. However, they house essential 
steps in the production of enzymatic cofactors, such as FeS clusters. Until recently it was generally accepted that there is no other organelle in the cell that can take over this function. However, a discovery of oxymonad Monocercomonoides sp., a eukaryotic organism without mitochondria, has changed this dogma. In this organism, the enzymes of the FeS cluster biosynthesis pathway were translocated to the cytoplasm and the mitochondria were consequently lost (Karnkowska et al., 2016).

\subsubsection{Import of nuclear-encoded proteins into mitochondria}

Yeast mitochondria contain approximately 1000 different proteins within four distinct compartments - OMM, IMS, IMM and the matrix (Sickmann, 2003; Schmidt et al., 2010). 99\% of the mitochondrial proteome is encoded in the nuclear genome and only a handful of proteins (8 in S. cerevisiae) are produced "in house" (Ott and Herrmann, 2010). Thus, mitochondria depend greatly on cytosolic translation and the correct targeting and import of nuclear-encoded proteins (Chacinska et al., 2009). In most cases, mitochondrial proteins are targeted to the organelle by a presequence, an amphipatic $\alpha$-helix that varies greatly in length and amino acid composition (Schatz and Dobberstein, 1996; Vögtle et al., 2009; Schulz et al., 2015), or by internal hydrophobic recognition sites, common for multispanning membrane and soluble IMS proteins (Chacinska et al., 2009). Independent of how the proteins are targeted, they are imported into the organelle in an unfolded state and therefore guided by cytoplasmic chaperones to prevent their aggregation (Young et al., 2003). Although it is generally accepted that proteins are imported into the mitochondria post-translationally, recent data suggests that co-translational protein import might also be possible (Wiliams et al., 2014). Five major import pathways exist that deliver proteins to mitochondria.

Translocase of the Outer Membrane, TOM. The TOM complex is the main entry gate to the mitochondria that delivers substrate proteins to at least four different downstream import machineries (Ryan et al., 2000). Tom20 and Tom22 are protein-receptors that recognize N-terminal presequences, whereas Tom70 recognizes the internal hydrophobic signals of metabolite carriers. However, Tom70 was recently shown to interact with the presequence of Mdl1 and therefore to act as a presequence-receptor (Melin et al., 2015). Receptor proteins pass on their substrates to Tom40, a $\beta$-barrel channel in the OMM. The inner side of Tom40 has both negatively charged and hydrophobic residues, which interact with positively charged presequences and hydrophobic signals of imported substrates, respectively (Shiota et al., 2015).

Translocase of the Inner Membrane, TIM23. The central core of the TIM23 complex, Tim23, forms a channel in the IMM (Truscott et al., 2001) that mediates both protein insertion into the IMM and translocation to the matrix. Whereas membrane protein insertion requires only the membrane potential (van der 
Laan et al., 2007), additional energy from ATP hydrolysis is needed for matrix translocation. mtHsp70 assists ATP-driven protein translocation through the Tim23 channel (Lang et al., 1999) and functions together with the Presequence translocase - Associated import Motor (PAM), which consists of the Pam18, Pam16, Pam17, Mge1 and Tim44 proteins.

TIM22. The TIM22 complex inserts proteins with internal targeting signals, such as members of the mitochondrial metabolite-carrier family, into the IMM (Rehling et al., 2004). The substrates first pass through the Tom40 channel and are guided to TIM22 by small chaperones of the IMS that prevent substrate aggregation (Koehler et al., 1998). The chaperones dock their substrates onto TIM22 (Wagner et al., 2008), a pore-forming dimer, which then opens in a membrane potentialdependent manner to accommodate $\alpha$-helices and mediate membrane protein insertion (Rehling et al., 2003). Recently, in yeast, subunit 3 (Sdh3) of the complex II of the respiratory chain was shown to cooperate with TIM22 in the biogenesis and assembly of membrane proteins, implying that subunits of the respiratory chain can have dual functions (Gebert et al., 2011).

Mitochondrial IMS Assembly (MIA) pathway Proteins destined for the IMS contain multiple disulfide bridges and a characteristic motif, called the IMS sorting signal (MISS) (Sideris et al., 2009). These proteins are properly folded in the IMS with the help of MIA machinery, consisting of Mia40 together with the sulfhydril oxidase Erv1 (Mesecke et al., 2005).

Sorting and Assembly machinery of the outer Membrane, SAM. The outer mitochondrial membrane contains a unique type of protein that originates from a prokaryotic ancestor - integral $\beta$-barrels. These proteins are initially imported via the TOM complex to the mitochondrial IMS, where they associate with small chaperones to prevent their aggregation. The final step of membrane insertion is mediated by the SAM complex, consisting of a channel-forming Sam50, together with Sam35 and Sam37 (Wiedemann et al., 2003).

The Mitochondrial import complex, MIM. Mim1 was found to assist the insertion of OMM proteins anchored to the membrane by a single N-terminal $\alpha$-helix, like Tom20 and Tom70, and multispanning membrane proteins, like Ugo1 (Becker et al., 2008; Papic et al., 2011). Interestingly, the biogenesis of the C-tail anchored proteins of the OMM is not understood and the protein machinery for their insertion has not yet been identified. However, it is known that the lipid composition of the membrane is essential for their insertion (Kemper et al., 2008).

Non-canonical pathways for protein import. Evidence has emerged that some proteins do not use any of the 5 known canonical import machineries for their import, but rather a combination of several. One example is the outer membrane protein, Om45, that contains an N-terminal membrane anchor and a large IMSexposed domain. Om45 is first imported into the mitochondrion in a membrane- 
potential-dependent manner via the TOM and TIM23 complexes. However, at some point Om45 escapes these translocases and is inserted into the OMM by MIM (Song et al., 2014; Wenz et al., 2014).

\subsection{Expression of the mitochondrial genome}

\subsubsection{The mitochondrial genome}

In the course of evolution, eukaryotic organisms have retained a mitochondrial genome (mtDNA). The reason for this is still a matter of debate. Replication and transcription of mtDNA, as well as the translation of the produced mRNAs requires the import of hundreds of proteins from the cytoplasm and consumes enormous cellular resources. Two major theories dominate in the field that explain why not all mitochondrial genes were transferred to the nucleus. The hydrophobicity theory suggests that mitochondrial proteins, if translated in the cytoplasm, would not be efficiently imported into the mitochondria because of their high hydrophobicity (von Heijne, 1986). The co-location for redox regulation hypothesis claims that retaining a genome inside of the organelle allows it to regulate gene expression according to its redox state (Allen, 1993).

The mitochondrial genomes among eukaryotes are extremely diverse and encode for different protein-components of the OXPHOS machinery. The yeast S.cerevisiae is an important model organism for which manipulation of the mtDNA is possible and, therefore, its gene-expression system is relatively well-characterized. In the following section, maintenance of the $S$. cerevisiae genome, as well as the biogenesis of mitochondrial-encoded proteins, will be discussed in more detail.

mtDNA is packed together with associated proteins into mitochondrial nucleoids that vary in size from 0.2 to $0.9 \mu \mathrm{m}$. Depending on whether cells were cultured aerobically or anaerobically, there can be as many as 60 to 8 nucleoids present in one cell, respectively, with each nucleoid containing from 1 to 20 copies of mtDNA (Miyakawa et al., 1987; Shiiba et al., 1997). mtDNA is packed by positively charged non-histone proteins of High Mobility Group family (HMG) (Caron et al., 1979; Diffley and Stillman, 1991). Abf2 is the best studied HMG protein in yeast. It was estimated that 1 molecule of Abf2 is present per 15-30 bp of mtDNA (Brewer et al., 2003). The absence of Abf2 leads to yeast mitochondrial genome instability, but not to the complete loss of mitochondrial nucleoids (Newman et al., 1996), meaning that there are more proteins involved in nucleoid packaging.

Yeast mtDNA encodes for 2 rRNAs (15S rRNA and 21S rRNA), 24 tRNAs, 7 mRNAs, and the 9S RNA component of RNase P (Foury et al., 1998) (Fig. 1.2). 8 proteins are encoded by mtDNA in total, 7 of them are components of the respiratory chain and one is a structural component of a small ribosomal subunit (Var1). Depending on the laboratory strain, yeast mtDNA contains up to 13 introns (9 of 


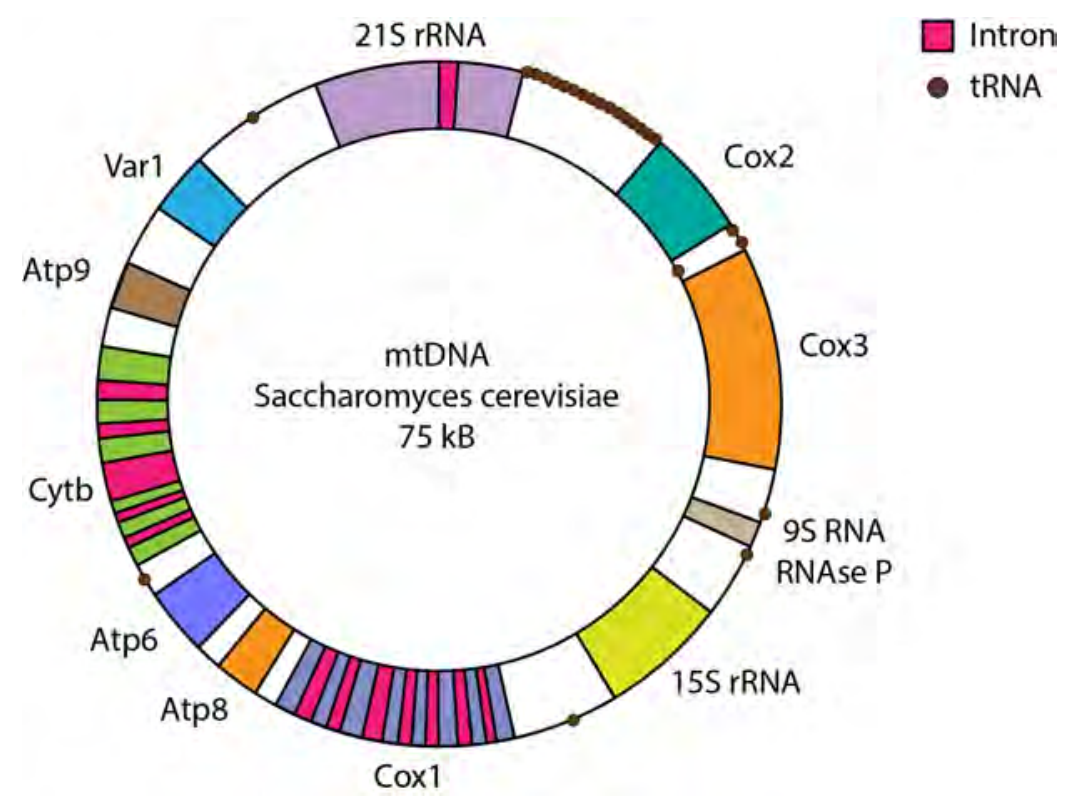

Figure 1.2 Organisation of the S. cerevisiae mitochondrial genome. The yeast mitochondrial genome encodes for 2 rRNAs, 24 tRNAs, 7 mRNAs and 9S RNA component of RNase P. Genes encoding for Cox1, Cytb and 21S rRNA contain introns. rRNA, ribosmal RNA; tRNA, transfer RNA. Adapted from Jacobs, 2001.

group I and 4 of group II) within the COX1, COB and $21 S$ rRNA genes (Pel and Grivell, 1993). Intronic ORFs encode endonucleases, maturases and reverse transcriptases required for splicing and transcript maturation. Due to long noncoding regions, the yeast mitochondrial genome is $75 \mathrm{kB}$ in length and is almost five times bigger than the human mitochondrial genome.

\subsubsection{Mitochondrial transcription and maturation of transcripts}

Transcription in mitochondria is performed by a nuclear-encoded mitochondrial RNA polymerase (mtRNAP), consisting of the catalytic subunit Rpo41p and the transcription factor Mtf1p (Greenleaf et al., 1986; Jang and Jaehning, 1991). Rpo41p is related to bacteriophage polymerases and therefore originated from neither the endosymbiont nor the host cell (Shutt and Gray, 2006). mtRNAP binds to simple nonanucleotide promoter sequences to initiate the transcription of 13 different polycistronic primary transcripts (Osinga et al., 1982; Christianson and Rabinowitz, 1983). The mechanism of transcriptional termination in yeast mitochondria remains unknown.

In $S$. cerevisiae, transcription in mitochondria is controlled by ATP levels as all transcripts start with an adenine nucleotide (Amiott and Jaehning, 1983). However, the regulation of mitochondrial gene expression happens mainly at the posttranscriptional level, during transcript processing and translation, and involves 
Table 1.1 Factors involved in the expression of the $S$. cerevisiae genome

\begin{tabular}{|c|c|c|}
\hline Gene & Protein function & References \\
\hline$A E P 1$ & Stability and expression of $A T P 9$ mRNA & $\begin{array}{l}\text { Payne et al., 1993; Ziaja et al., } \\
1993\end{array}$ \\
\hline$A E P \mathscr{2}$ & Stability and translation of $A T P 9$ mRNA & Finnegan et al., 1995 \\
\hline AEP3 & Stability and expression of $A T P 6 / 8$ mRNA & Ellis et al., 2004 \\
\hline АТР22 & Translation of Atp6 & Zeng et al., 2007a \\
\hline ATP25 & Stability of ATP9 mRNA and Atp9 ring assembly & Zeng et al., 2008 \\
\hline$C B P 1$ & 5' end processing of $C O B$ mRNA, together with Pet127 & $\begin{array}{l}\text { Dieckmann et al., 1982; Dieck- } \\
\text { mann et al., } 1984\end{array}$ \\
\hline$C B P 2$ & $\begin{array}{l}\text { Splicing of the bi5 and bi } 2 \text { group I introns of } C O B \text { mRNA } \\
\text { precursor }\end{array}$ & Gampel et al., 1989 \\
\hline$C B P 3$ & Expression and assembly of Cytb & Gruschke et al., 2011; \\
\hline CBP6 & Expression and assembly of Cytb & $\begin{array}{l}\text { Gruschke et al., 2011; Dieck- } \\
\text { mann and Tzagoloff, } 1985\end{array}$ \\
\hline$C B S 1$ & Translation of the $C O B$ mRNA & Rödel, 1986 \\
\hline$C B S 2$ & Translation of the $C O B$ mRNA & Rödel, 1986 \\
\hline$C B T 1$ & 5 ' end processing of the $C O B$ mRNA & Rieger et al., 1997 \\
\hline COA3 & $\begin{array}{l}\text { Translation of COX1 mRNA and assembly of complex } \\
\text { IV }\end{array}$ & Mick et al., 2010 \\
\hline COX14 & $\begin{array}{l}\text { Translation of the COX1 mRNA and assembly of Com- } \\
\text { plex IV }\end{array}$ & Barrientos et al., 2004 \\
\hline МАМЗ3 & Translational activator for $C O X 1 \mathrm{mRNA}$ & $\begin{array}{l}\text { Seytter et al., 1998; Roloff and } \\
\text { Henry, } 2015\end{array}$ \\
\hline$M R S 1$ & $\begin{array}{l}\text { Splicing of the bi } 3 \text { intron of } C O B \text { mRNA and the ai } 5 \beta \\
\text { intron of } C O X 1 \mathrm{mRNA}\end{array}$ & Bousquet et al., 1990 \\
\hline MSS18 & Splicing of the ai $5 \beta$ intron of $C O X 1$ mRNA & \\
\hline$M S S 51$ & Translation of $C O X 1 \mathrm{mRNA}$ & $\begin{array}{l}\text { Barrientos et al., 2004; Perez- } \\
\text { Martinez et al., } 2003\end{array}$ \\
\hline$M S S 116$ & $\begin{array}{l}\text { Splicing of group I and II introns from } C O X 1 \text { and } C O B \\
\text { mRNAs }\end{array}$ & Huang et al., 2004 \\
\hline NCA2 & Processing and expression of the $A T P 6 / 8 \mathrm{mRNA}$ & Camougrand et al., 1995 \\
\hline$N C A 3$ & Processing and expression of the $A T P 6 / 8 \mathrm{mRNA}$ & Pelissier et al., 1995 \\
\hline PET111 & Translation of the COX2 mRNA & Green-Willms et al., 2001 \\
\hline PET122 & Translation of the $C O X 3 \mathrm{mRNA}$ & Naithani et al., 2003 \\
\hline PET309 & $\begin{array}{l}\text { Stability of intron-containing transcripts and translation } \\
\text { of the } C O X 1 \mathrm{mRNA}\end{array}$ & Manthey and McEwen, 1995 \\
\hline PET494 & Translation of the $C O X 3 \mathrm{mRNA}$ & $\begin{array}{l}\text { Müller and Fox, 1984; Naithani } \\
\text { et al., } 2003\end{array}$ \\
\hline PET54 & $\begin{array}{l}\text { Translation of the } C O X 3 \mathrm{mRNA} \text { and splicing of the ai5 } \beta \\
\text { intron of } C O X 1 \mathrm{mRNA}\end{array}$ & $\begin{array}{l}\text { Costanzo et al., 1989; Valen- } \\
\text { cik et al., 1989; Naithani et al., } \\
\text { 2003; Mayorga et al., } 2016\end{array}$ \\
\hline QRI5 & $\begin{array}{l}\text { Processing of intron-containing transcripts and transla- } \\
\text { tion of the COX } 1 \mathrm{mRNA}\end{array}$ & Barros et al., 2006 \\
\hline$R M D 9$ & $\begin{array}{l}\text { Mitochondrial protein with role in delivering mRNAs to } \\
\text { ribosomes }\end{array}$ & Williams et al., 2007 \\
\hline
\end{tabular}

Continued on the next page 
Table 1.1 - Continued from previous page

\begin{tabular}{l|l|l}
\hline Gene & Protein function & References \\
\hline RSM28 & Mitochondrial ribosomal protein of the small subunit; & Williams et al., 2005; Williams \\
& possibly promotes translation initiation & et al., 2007 \\
SMT1 & Negative regulator of ATP6/8 mRNA translation & Rak et al., 2016 \\
SOV1 & Var1 expression & Sanchirico, 1998 \\
\hline \hline
\end{tabular}

Modified from Lipinski et al., 2010

almost exclusively nuclear-encoded proteins (summarized in Table 1.1). In brief, introns are spliced-out with the help of intron-encoded maturases and nuclear-encoded splicing factors, such as Mss18, Cbp2 and Mrs1. Afterwards, the mature 3' ends of the transcripts are formed by processing at a conserved dodecamer sequence (Osinga et al., 1984).

This cleavage, in some cases, physically separates mRNAs from the initial polycistronic transcripts. In other cases, open reading frames remain associated, as in the case of the bicistronic ATP6/ATP8 mRNA. The exact mechanism and machinery required for 3 ' end processing is not yet fully characterized. Interestingly, yeast mitochondria lack the poly $(\mathrm{A})$ polymerase and therefore do not polyadenylate mRNA precursors (Gagliardi et al., 2004). However, all yeast mitochondrial mRNAs contain long 5' untranslated regions (5' UTRs), which provide binding sites for nuclear-encoded, gene-specific, translational activators and proteins that regulate mRNA stability (Bonnefoy et al., 2001; Naithani et al., 2003; Green-Willms et al., 2001; Costanzo et al., 1989). tRNAs are excised from the polycistronic precursors and are further processed at their 3' and 5' ends (Chen and Martin, 1988; Stribinskis et al., 1996).

In yeast, the expression of the mitochondrial genome is balanced between transcription and RNA degradation. The degradation of mtRNAs is performed by the mitochondrial degradosome complex, consisting of RNA helicase Suv3 and exoribonuclease Dss1 (Dziembowski et al., 2003). Knockout of either protein leads to a similar phenotype - an accumulation of excised intronic sequences, non-processed transcripts and therefore a loss of mitochondrial translation.

\subsubsection{Mitochondrial translation}

It was initially believed that, due to its origin, the mitochondrial translation system should not differ significantly from that of bacteria. However, recent discoveries clearly showed that there is very little conservation between the two in regard to codon usage, mRNAs, tRNAs and ribosomal structure. In this section, the $S$. cerevisiae translation system will be discussed in more detail.

Mitochondrial tRNAs and mRNAs. Here, several major features of the mitochondrial translation systems will be mentioned: 
(I) The yeast mitochondrial genome encodes a full set of tRNAs, however, one nuclear-encoded lysine tRNA is imported into the mitochondrial matrix from the cytoplasm. Most likely, this tRNA has a regulatory function as it is not essential for mitochondrial protein translation (Schneider, 2011). Overall, mitochondria contain only 24 tRNAs (in comparison to more than 40 in bacteria) that correspond to 20 different amino acids, meaning that each tRNA needs to recognize additional codons (Wesolowski and Fukuhara, 1979).

(II) The genetic code, used by mitochondria, varies significantly from the canonical one (for example, leucine codons CUA, CUC, CUG and CUU are used for threonine).

(III) Mitochondrial mRNAs contain long 5' and 3' untranslated regions, important for translational regulation. Moreover, they lack a classical Shine-Dalgarno region for translation initiation and it is not really understood how the start codon is recognized (Dunstan et al., 1997; Christian and Spremulli, 2010). As has already been mentioned, yeast mitochondrial mRNAs are not polyadenylated at their 3' ends.

Mitochondrial ribosomes. Recently, high-resolution cryo-EM structures of a large ribosomal subunit, as well as of a complete mitochondrial ribosome, were published (Amunts et al., 2014; Desai et al., 2017). The large subunit of a mitochondrial ribosome $(54 \mathrm{~S})$ contains a $21 \mathrm{~S}$ rRNA and 39 proteins, 13 of which are unique to mitochondria. The small subunit (37S) consists of a $15 \mathrm{~S}$ rRNA and 34 proteins, including 14 without homologs in the evolutionarily-related bacterial ribosome. Therefore, mitochondrial ribosomes have an overall increased protein content compared to bacterial ribosomes, as well as a higher protein:RNA ratio (2:1 in mitochondria compared to 1:2 in prokaryotes). The most significant difference is the loss of the 5S rRNA in mitochondrial ribosomes. Normally, 5S rRNA forms a central protuberance on the large subunit (Dontsova and Dinman, 2005), but in yeast, the central protuberance is formed around the $21 \mathrm{~S}$ rRNA expansion segment. Despite all the structural differences, the catalytic centers contain conserved nucleotides, implying that the overall protein synthesis mechanism is conserved.

The translation cycle in mitochondria. Translation in mitochondria proceeds through conserved stages, called initiation, elongation and termination. Initiation is usually the rate-limiting and the most regulated step. Yeast mitochondrial initiation factor 2 (IF2) is encoded by the nuclear IFM1 gene. IF2 is the most evolutionary-conserved factor present in both cytoplasmic and mitochondrial translation systems in various species. IF2 binds initiator tRNA ${ }^{\mathrm{Met}}$ and positions it on a start-codon in a GTP-dependent manner. In yeast, mtDNA encodes for two different tRNA ${ }^{\mathrm{Met}}$, one for the initiation and another one for the elongation cycle. Despite the usage of a specialized initiator tRNA, its formylation in yeast mitochondria is not required (Garofalo et al., 2003). The yeast mitochondrial translation system dif- 
fers significantly from the cytosolic, as initiation of translation requires additional translational activators (TA). Each mRNA requires a specific nuclear-encoded TA (Table 1.1) that interacts with the 5' UTR of the corresponding mRNA and, usually, with the mitochondrial ribosome. Despite a significant amount of data about the role of TAs, the molecular details of their function remain unknown.

Two factors are involved in the yeast mitochondrial elongation cycle, Mef1 and Mef2, homologous to the prokaryotic elongation factors EF-G and EF-Tu, respectively (Vambutas et al., 1991). A bacterial homolog of EF-Ts is not present in $S$. cerevisiae, however, it was found in Schizosaccharomyces pombe and human mitochondria (Towpik, 2005). EF-Tu delivers the aminoacyl-tRNAs to the mitochondrial ribosome in complex with GTP, whereas EF-G catalyzes the coordinated movement of tRNA and mRNA during the elongation cycle.

Normally, termination occurs when a translating ribosome encounters one of the three stop codons - UGA, UAA, UAG. However, mitochondria have reduced the number of termination codons to only two, with UGA coding for tryptophan (Fox, 1979). The termination step is mediated by two factors, mitochondrial release factor $\mathrm{mRF} 1$ and ribosomal recycling factor RRF. mRF1 decodes the stop signal on mRNA and is a homolog of prokaryotic RF1 and RF2 (Pel et al., 1992).

\subsubsection{Co-translational membrane protein insertion}

The mitochondrial genome encodes seven membrane proteins of OXPHOS system. Most of them are polytopic membrane proteins that span the IMM up to 12 times (Cox1), with only Atp8 having a single transmembrane domain (Fig. 1.3). During insertion, proteins follow the positive-inside rule, similar to bacterial membrane proteins. This means that protein loops and termini in the matrix carry a net positive charge, whereas the soluble IMS domains are charged negatively (Ott and Herrmann, 2010).

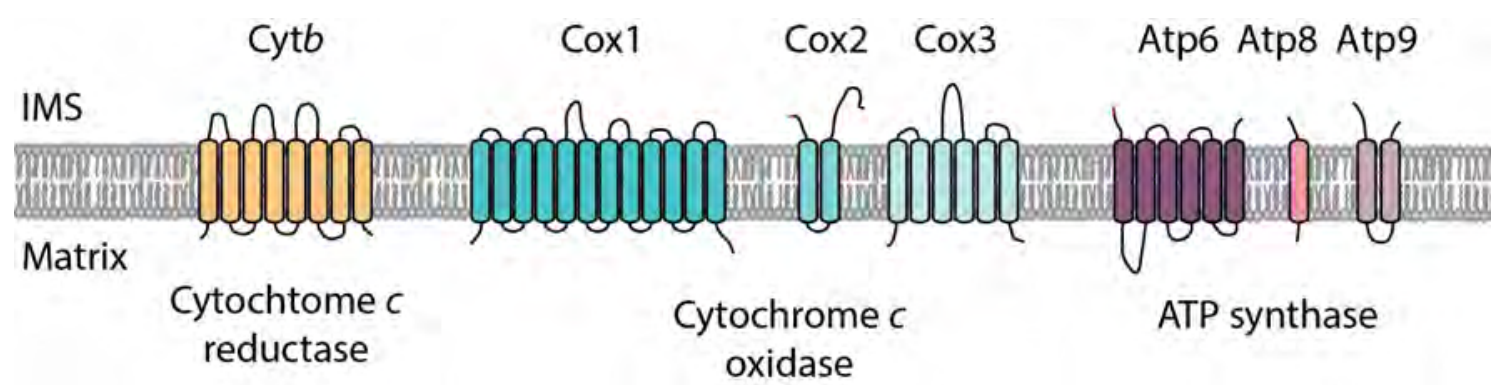

Figure 1.3 Mitochondrial-encoded membrane proteins of $\boldsymbol{S}$. cerevisiae. The yeast mitochondrial genome encodes for 7 protein components of the OXPHOS system. IMS, inter membrane space. Adapted from Ott and Herrmann, 2010.

The mitochondrial membrane protein insertion system is homologous to the bacterial machinery. The bacterial YidC complex usually assembles small substrates 
that lack highly charged domains, whereas the membrane insertion of larger proteins requires the SecYEG translocon (Nagamori et al., 2004; Driessen and Nouwen, 2008). The mitochondrial insertion system was simplified over the course of evolution and only the YidC homolog, Oxa1, is present in S. cerevisiae (Hell et al., 2001). Oxa1 is a member of the YidC/Alb3/Oxa1 family that represents proteins of bacterial membranes, chloroplast and inner mitochondrial membranes, respectively. Oxa1 contains a catalytic core composed of 5 transmembrane segments, similar to YidC (Kumazaki et al., 2014a; Kumazaki et al., 2014b). The protein was shown to dimerize (Kohler et al., 2009). An Oxa1 dimer, containing 10 transmembrane spans, resembles the bacterial SecY translocon and most likely is the actively translocating form of the channel, as dimerization allows the formation of a membrane-conducting channel, as well as the release of proteins into the membrane.

In yeast, Oxa1 directly binds to translating ribosomes via its C-terminus and therefore engages with nascent chains (Jia et al., 2003). Apart from mediating protein membrane insertion, Oxa1 is believed to facilitate the folding and assembly of membrane proteins. This suggestion came from an observation that in E. coli, subunit $c$ of the $\mathrm{F}_{1} \mathrm{~F}_{\mathrm{o}}$-ATP synthase (homolog of the mitochondrial Atp9 protein) is assembled into the mature enzyme with the help of Oxa1 homolog YidC (van der Laan et al., 2004) and was later proven to be the case in yeast (Jia et al., 2007). Surprisingly, only membrane insertion of the Cox2 protein strictly depends on Oxa1, whereas all other mitochondrial-encoded products are integrated into the membrane even when Oxa1 is absent (Hell et al., 2001; Bauer et al., 1994). Therefore, multiple genetic screens were performed in order to identify additional components of the mitochondrial insertion machinery.

Cox18 is one more member of the YidC/Alb3/Oxa1 family, found in yeast mitochondria. It contains the conserved insertase domain but, unlike Oxa1, does not have the C-terminus required for the interaction with ribosomes (Souza et al., 2000; Saracco and Fox, 2002; van Bloois et al., 2007). Cox18 was proposed to play an important role in the assembly of the C-terminus of Cox 2 and, therefore, to be involved in cytochrome $c$ oxidase biogenesis.

To decrease the probability of nascent chain aggregation, translation in mitochondria is coupled to membrane protein insertion. As a result, actively translating mitochondrial ribosomes are associated with the IMM. In yeast, a nuclear-encoded Mba1 protein mediates ribosomal attachment to the membrane insertion machinery (Preuss et al., 2001; Ott et al., 2006). Cryo-electron tomography studies have shown that Mba1 is aligned with the tunnel exit to provide an uninterrupted route for the nascent chain from the ribosome to the membrane (Pfeffer et al., 2015). Although Mba1 is not an essential protein, a simultaneous deletion of MBA1 and the C-terminus of Oxa1 leads to a significant impairment of co-translational protein insertion. 
The second mitochondrial ribosomal tether is Mdm38, a $65 \mathrm{kDa}$ protein that was identified in a systematic genome-wide screen for genes important for mitochondrial distribution and morphology (MDM) (Dimmer et al., 2002). Mdm38 was found to associate with mitochondrial ribosomes (Frazier et al., 2006). Deletion of MDM38 leads to a severe respiration defect and altered mitochondrial morphology. The observation that Mdm38 associates with newly-translated mitochondrially encoded proteins, together with impaired Atp6 and Cyt $b$ insertion into the inner mitochondrial membrane upon MDM38 deletion, makes it a likely candidate to facilitate ribosomal attachment to the IMM (Frazier et al., 2006). Moreover, it was shown that simultaneous deletion of MBA1 and MDM38 results in yeast synthetic lethality on non-fermentable carbon sources, suggesting overlapping functions of Mba1 and Mdm38 for biogenesis of the respiratory chain (Bauerschmitt et al., 2010).

\subsection{Mitochondrial Oxidative Phosphorylation System}

\subsubsection{Structure and function of OXPHOS system}

The mitochondrion is the main source of energy in the eukaryotic cell. It converts energy from reducing equivalents $\left(\mathrm{NADH}, \mathrm{FADH}_{2}\right)$ into the form of highenergy ATP bonds. Five different enzyme complexes located in the IMM drive this process (Fig. 1.4). Complexes I, III and IV transfer electrons to acceptor molecules and pump protons from the matrix into the IMS, generating a proton gradient. The last enzyme complex, complex $\mathrm{V}$ (or $\mathrm{F}_{1} \mathrm{~F}_{\mathrm{o}}$-ATP synthase), uses this proton gradient to generate ATP from ADP and $\mathrm{P}_{\mathrm{i}}$. All enzymes involved are composed of multiple subunits of different genetic origin. Assembly of these complexes is a multistep process that requires numerous assembly factors and a sophisticated regulation mechanism.

There are two ways for electrons to enter the electron transport chain. The first is to be transferred from NADH, through complex I (NADH:ubiquinone oxidoreductase), to ubiquinone. The second is a transfer from succinate to complex II (succinate:ubiquinone oxidoreductase) and then to ubiquinone. Electrons travel from ubiquinone, through complex III (ubiquinol:ferricytochrome $c$ oxidoreductase), to cytochrome $c$ and, finally, through complex IV (cytochrome $c$ oxidase) to oxygen. Only electron transfer through complex II is not coupled with proton pumping to the IMS.

Complex I (NADH:ubiquinone oxidoreductase or NADH dehydrogenase) is the first proton-pumping complex of the respiratory chain. It transfers 2 electrons from $\mathrm{NADH}$ and pumps 4 protons from the matrix to the IMS. One molecule of flavin mononucleotide and several iron-sulfur clusters participate in the redox reactions catalyzed by NADH dehydrogenase (Weiss et al., 1991). S. cerevisiae does not have complex I. Instead, it uses an alternative enzyme, FAD rotenone-insensitive 


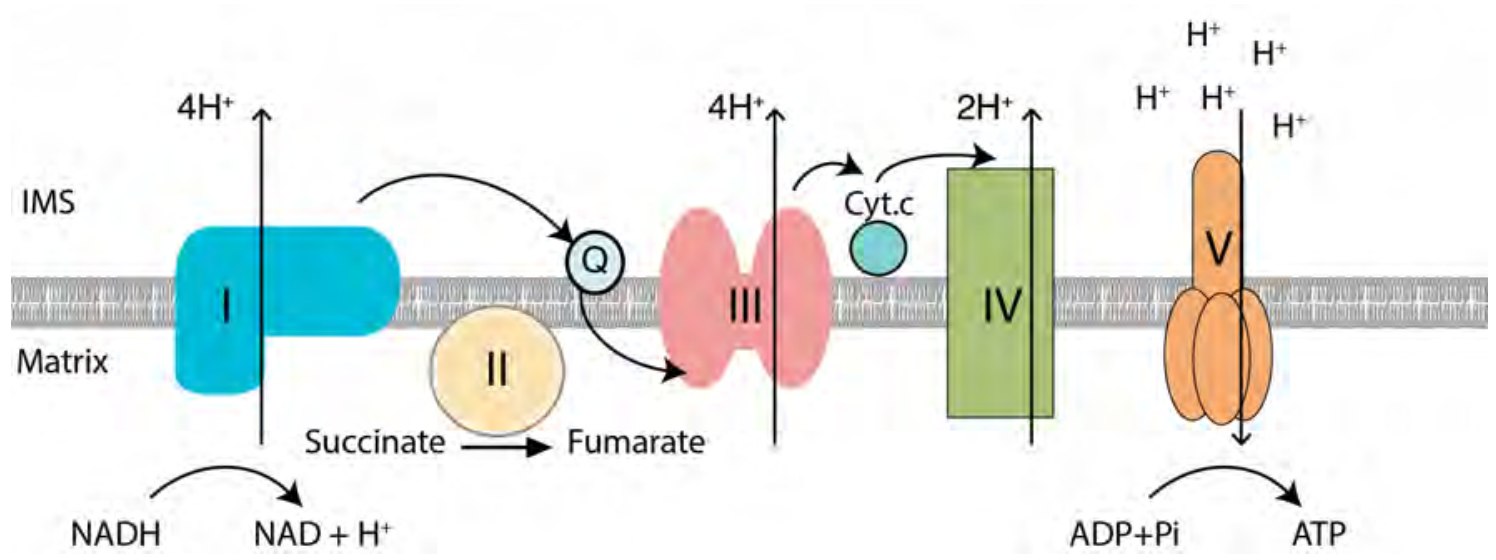

Figure 1.4 Mitochondrial OXPHOS system. The oxidation-phosphorylation system consists of four complexes of the respiratory chain (I-IV) and complex V, or $\mathrm{F}_{1} \mathrm{~F}_{\mathrm{o}}-\mathrm{ATP}$ synthase. Electrons are passed from NADH and $\mathrm{FADH}_{2}$ to molecular oxygen in a series of reactions to produce ATP. Q, ubiquinone.

dehydrogenase that has a single nuclear-encoded subunit and does not possess a proton-pumping function (de Vries and Grievell, 1988; Lenaz and Genova, 2010). Neurospora crassa was initially used as a eukaryotic model to study complex I structure. It was shown that the complex consists of two parts orientated perpendicular to each other in an overall L-shaped structure (Guenebaut et al., 1997). Moreover, the recently published crystal structure of Yarrowia lipolitica complex I provided valuable insights into the structure and mechanism of complex I function (Zickermann et al., 2015).

Succinate:ubiquinone oxidoreductase, or succinate dehydrogenase (SDH, complex II), is highly conserved among all aerobic organisms and is a membrane-bound enzyme of the Krebs cycle that catalyzes the oxidation of succinate to fumarate and transfers electrons to ubiquinone. In contrast to other enzymes of the respiratory chain, complex II does not contain structural subunits encoded in the mitochondrial genome. SDH consists of 4 polypeptides, a FAD moiety and four iron-sulfur clusters in the redox center. SDH transfers 2 electrons to ubiquinone and is considered to be the smallest enzyme of the respiratory chain (Hatefi, 1985, Lenaz and Genova, 2010).

Ubiquinone is a small hydrophobic mobile electron carrier, reduced by complexes I and II, and oxidized by complex III of the respiratory chain during electron transfer (Genova and Lenaz, 2011). Ubiquinone can exist in three redox states: fully oxidized (ubiquinone), semiquinone (ubisemiquinone) and fully reduced (ubiquinol). Complex III, or ubiquinol-cytochrome $c$ oxidoreductase ( $b c_{1}$ complex), accepts electrons from ubiquinol (Hatefi, 1985). In S. cerevisiae, this complex consists of 10 different subunits: three respiratory subunits (cytochrome $b$, cytochrome $c$ and Rieske iron-sulfur protein); 2 core proteins (Cor1 and Qcr1) and 5 additional lowmolecular weight proteins. Only cytochrome $b$ is encoded by the mitochondrial $c o b$ 
gene (Tzagoloff, 1995). Complex III passes electrons to another mobile electron carrier, called cytochrome $c$.

Cytochrome $c$ belongs to the cytochrome protein family but, in contrast to other members of this family, it is hydrophilic and loosely associated with the inner mitochondrial membrane. The main function of cytochrome $c$ is to shuttle electrons between complex III and complex IV of the respiratory chain (Volkov and van Nuland, 2012).

The heme-copper oxygen reductase - cytochrome $c$ oxidase (COX), or complex $\mathrm{IV}$, is the last enzyme in the respiratory chain. It transfers electrons to molecular oxygen, thereby generating water, oxidizing cytochrome $c$ and pumping protons across the IMM (Capaldi, 1990). Yeast complex IV contains 11 different subunits. The three largest subunits (Cox1, Cox2 and Cox3) are mitochondrial-encoded. The other 8 are synthesized in the cytoplasm and imported by the TOM/TIM23 import machineries (Mick et al., 2011). Genetic studies suggest that mitochondrial-encoded proteins form the catalytic core of the enzyme, while nuclear-encoded proteins either participate in complex assembly or modulate its catalysis and stability. Studies in $S$. cerevisiae have revealed that more than 41 specific genes are additionally required for the assembly and maturation of cytochrome $c$ oxidase (Poynton et al., 1995; Carr and Winge, 2003; Mick et al., 2011).

Respiratory chain complexes from S. cerevisiae, and other higher eukaryotes, form higher-order structures called supercomplexes or respirasomes, whose structure and assembly mechanisms were recently revealed (Mileykovskaya et al., 2012; Letts et al., 2016; Cogliati et al., 2016; Wu et al., 2016). Respirasomes are advantageous to the cell by increasing the efficiency of electron transfer and reducing the production of reactive oxygen species (ROS) (Lenaz et al., 2010; Schägger, 2001). Supercomplexes can be easily visualized using Blue Native PAGE protein separation after mitochondrial solubilization using a mild detergent (Schägger and Jagow, 1991).

\subsubsection{Biogenesis of complex III}

Complexes of the respiratory chain assemble from subunits of two different genetic origins. Therefore, the coordinated expression of nuclear and mitochondrial genomes is required for successful OXPHOS system biogenesis. Yeast cells have developed a sophisticated mechanism for the assembly of complex III and IV, which includes a feedback-loop communication between mitochondrial translation and assembly.

Ubiquinol-cytochrome $c$ oxidoreductase is the first complex of the respiratory chain in yeast that couples electron transfer to proton translocation across the inner mitochondrial membrane. It consists of ten proteins, nine being encoded in the nucleus and one, cytochrome $b$, being mitochondrial-encoded. In this section, biogenesis of the yeast cytochrome $c$ reductase will be discussed; particular attention 
will be paid to the role of $\mathrm{Cbp} 3 / \mathrm{Cbp} 6$ and $\mathrm{Cbp} 4$ proteins in its biogenesis.

The cytochrome $b$ mRNA precursor is synthesized with 3 introns (bl2, bl3 and bl4) that belong to groups I and II. The latter means that the introns themselves possess catalytic activity required for their self-excision in vitro. In vivo, however, additional proteins are needed to stabilize RNA structure and to promote efficient splicing. Such proteins are Mss116, a protein with an RNA chaperone function that belongs to the DEAD-box protein family, Cbp2 and Mrs1 (Huang et al., 2004; Gampel et al., 1989; Bousquet et al., 1990). The mature COB mRNA is stabilized by the Cbp1 protein. It was shown that in the absence of Cbp1, COB mRNA levels are drastically decreased and, therefore, cytochrome $b$ is not translated (Dieckmann et al., 1982). An additional function for Cbp1 was proposed, namely the processing of the 5' UTR of $C O B$ mRNA during its maturation (Dieckmann et al., 1984).

Expression of cytochrome $b$ requires several factors. Cbs1 and Cbs2 were shown to interact with the 5' UTR of $C O B$ mRNA to activate its translation (Rödel, 1986). Interestingly, when the COB 5' UTR was exchanged to the 5' UTR of ATP9 mRNA, Cbs1 and Cbs2 were no longer required for efficient cytochrome $b$ translation. In line with this, when the 5' UTR of $C O X 3 \mathrm{mRNA}$, encoding cytochrome $c$ oxidase subunit 3, was exchanged to the 5' UTR of $C O B$ transcript, Cox3 expression became strictly Cbs1-dependent (Rödel and Fox, 1987). Both observations imply that untranslated regions of mitochondrial mRNAs contain specific recognition elements, required for binding of gene-specific translational activators (Rödel et al., 1985; Rödel, 1986). Additionally, Cbs1 and Cbs2 interact with mitochondrial ribosomes, but not with the newly translated cytochrome $b$, most probably to bringe the ribosome and the mRNA together (Kehrein et al., 2015).

Two more factors are required for efficient cytochrome $b$ biogenesis. Cbp3 and Cbp6 are involved in a feedback loop mechanism required for the coordination of cytochrome $b$ synthesis and assembly (Dieckmann and Tzagoloff, 1985; Wu et al., 2016; Gruschke et al., 2011; Gruschke et al., 2012). Deletion of CBP3 and CBP6 results in impaired yeast respiration due to the lack of mature complex III. Interestingly, upon $C B P 3 / C B P 6$ deletion, cytochrome $b$ is still translated, although at a reduced rate, but its stability is significantly decreased, most probably due to the impaired assembly with the nuclear-encoded subunits. Translation and stability of other mitochondrial-encoded OXPHOS machinery subunits is not affected in $c b p$ mutants (Gruschke et al., 2011).

Cbp3 and Cbp6 form a stable dimer that localizes to the mitochondrial matrix. The dimer associates peripherally with the inner mitochondrial membrane and with a mitochondrial ribosome near the peptide exit tunnel. Therefore, the Cbp3/Cbp6 dimer associates with newly translated cytochrome $b$ to prevent it from degradation and to assist it during further assembly steps. Before any structural subunits join the assembly pipeline, cytochrome $b$ binds Cbp3, Cbp6 and a membrane-bound assembly 
factor Cbp4 (Fig. 1.5). This first assembly intermediate, called intermediate I, accumulates in wild type cells to levels easily detectable by Western blotting and most likely represents a pool of cytochrome $b$ that is ready to assemble into complex III.

One of the crucial steps in cytochrome $b$ biogenesis is hemylation. Cytochrome $b$ contains two hemes; $b_{H}$, located closer to the matrix side, and $b_{L}$, incorporated into the IMS side of the IMM membrane (Yun et al., 1991). The two hemes are coordinated by histidine residues in a four-helix bundle (Yun et al., 1991; Hunte et al., 2000). Their incorporation into cytochrome $b$ is an essential step and an important check-point in the biogenesis process. Recently, the hemylation of cytochrome $b$ during its assembly was described (Hildenbeutel et al., 2014). It was shown that $b_{L}$ is the first site to be hemylated. Heme incorporation happens when cytochrome $b$ is associated with Cbp3 and Cbp6 before the formation of intermediate I. An efficient first hemylation event allows Cbp4 to join the assembly and intermediate I to be formed. The authors proposed that insertion of $b_{L}$ heme induces conformational changes in cytochrome $b$ that promote Cbp4 association. Cbp4 binding, in turn, induces changes in cytochrome $b$ structure, necessary for the second hemylation event. The hemylation of the $b_{H}$ site happens when cytochrome $b$ is a part of intermediate I. Importantly, successful second hemylation triggers association of cytochrome $b$ with the first structural subunits, Qcr7 and Qcr8, and releases the Cbp3/Cbp6 dimer. Cbp3/Cbp6 release is an important trigger that allows complex III assembly to proceed further. Moreover, it is essential for the reactivation of cytochrome $b$ translation as the $\mathrm{Cbp} 3 / \mathrm{Cbp} 6$ dimer relocates to the ribosome to activate cytochrome $b$ synthesis. In case hemylation fails, Cbp3 and Cbp6 remain trapped in intermediate I, ensuring that no more cytochrome $b$ is synthesized. Such a feedback loop is also activated if delivery of nuclear-encoded complex III subunits, namely Qcr7, Qcr8, Cor1 and Cor2, fails. This mechanism couples the translation of cytochrome $b$ with its assembly to ensure their coordination.

After Qcr7 and Qcr8 are assembled and the Cbp3/Cbp6 dimer is released, intermediate II is formed (Fig. 1.5). Importantly, Cbp4 remains associated with it until Cor1 and Cor2 are added to give rise to intermediate III. In intermediate III, no more assembly factors are present as cytochrome $b$ is already efficiently stabilized by structural subunits. Next, the second catalytically active subunit, Cyt $c_{1}$, is added together with Qcr6, to produce intermediate IV. Intermediate IV, as well as intermediate I, accumulate to detectable amounts in wild type cells and was previously described as a $500 \mathrm{kDa}$ complex (Zara et al., 2007). 


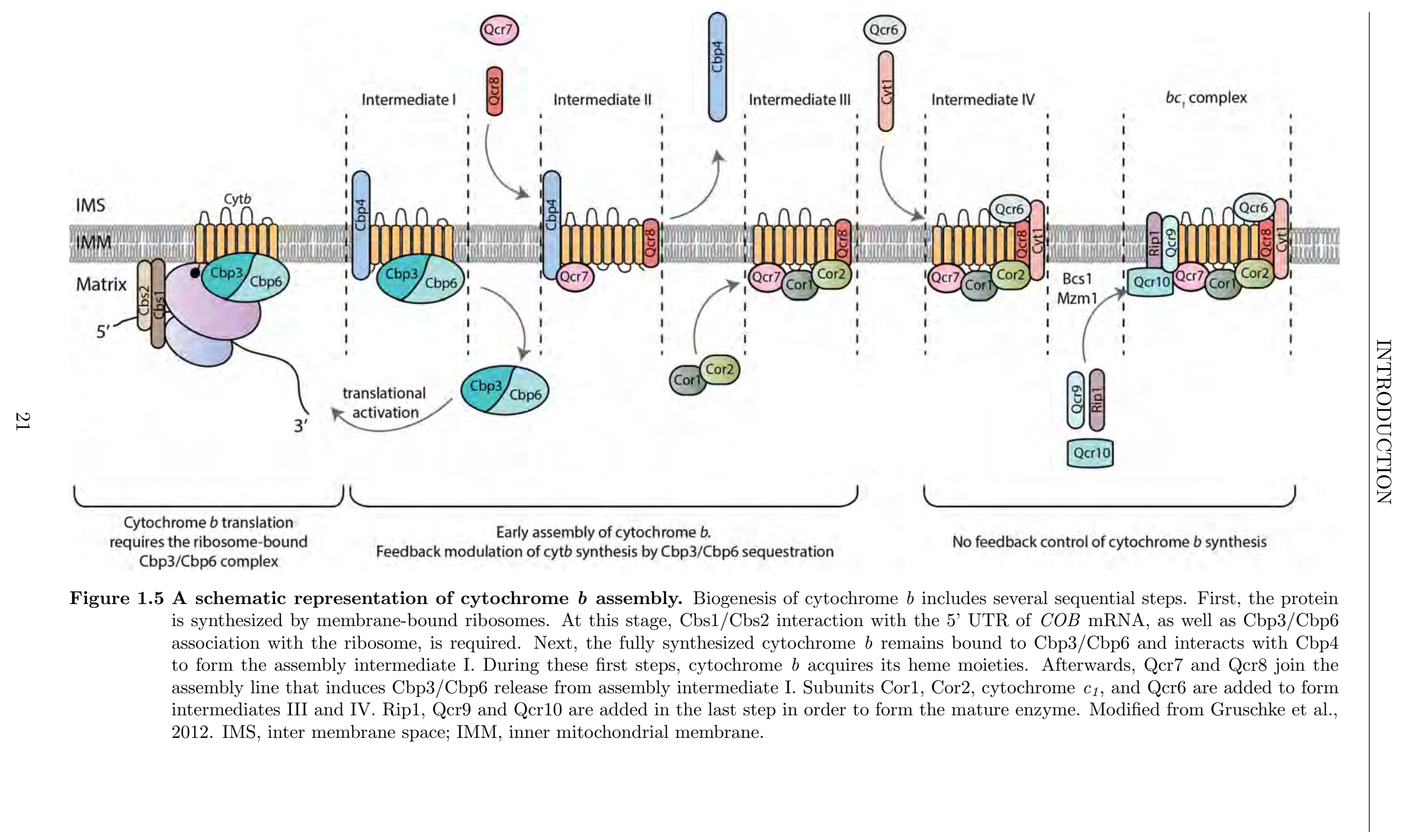


The last catalytic subunit of complex III, Rip1, is added together with two nonessential subunits, Qcr9 and Qcr10, to form the mature enzyme. Interestingly, Rip1 biogenesis requires specialized factors. First, Mzm1 interacts with Rip1 to stabilize its FeS clusters and, most likely, to facilitate its association with Bcs1 (Atkinson et al., 2011). Bcs1 is a AAA-protease that binds Rip1 and transports it across the IMM, which represents a unique pathway of mitochondrial protein translocation (Wagener et al., 2011).

The fully assembled complex III homodimerizes in the inner mitochondrial membrane (Xia et al., 1997; Hunte et al., 2000) and is never present in wild type cells in a monomeric form. It is not clear when exactly the dimerization occurs and whether there are additional factors involved in this process. Complex III dimers associate with either one or two copies of mature cytochrome $c$ oxidase (complex IV) to form supercomplexes of the respiratory chain (Cruciat et al., 1999; Heinemeyer et al., 2007; Vukotic et al., 2012). This presumably facilitates catalytic reactions performed by the complexes and additionally stabilizes them in the IMM (Vonck and Schäfer, 2009).

\subsubsection{Structure and function of the yeast $F_{1} F_{o}-A T P$ synthase}

$\mathrm{F}_{1} \mathrm{~F}_{\mathrm{o}}$-ATP synthases (in short, ATP synthases or complex V) is a group of enzymes that couples ATP synthesis/hydrolysis to the electrochemical membrane potential, which is built up by either protons or sodium ions across biological membranes. These complexes are present in bacteria, mitochondria and plant chloroplasts and share a common structure. The mitochondrial ATP synthase subunit composition and the mechanism of ATP synthesis are very much conserved among various eukaryotic species. Organisms differ only in their accessory subunits that either modify the enzyme's structure or have a regulatory function. Yeast ATP synthase consists of approximately 20 proteins, 7 of them are nonessential as they only regulate the oligomeric state of the enzyme or modulate its function (Table 1.2) (Arnold et al., 1998; Wittig and Schägger, 2008). Among the 13 essential proteins, 10 are encoded on the nuclear genome and 3 (Atp6, Atp8 and Atp9) are mitochondrial-encoded.

The mitochondrial ATP synthase can be subdivided into two major parts - a soluble $\mathrm{F}_{1}$, which has catalytic activity, and a hydrophobic membrane-bound $\mathrm{F}_{\mathrm{o}}$ part, which translocates protons across the IMM and transmits the energy from this translocation to $\mathrm{F}_{1}$. The $\mathrm{F}_{1}$ module consists of a catalytic head and a central stalk (Fig. 1.6). The catalytic head is a hexamer composed of a protein dimer occurring in three copies. Each dimer contains Atp1 $\left(F_{1} \alpha\right)$ and Atp2 $\left(F_{1} \beta\right)$ and possesses a nucleotide binding pocket (Stock et al., 1999). The $\mathrm{F}_{\mathrm{o}}$ part consists of a hydrophobic ring made from 10 molecules of Atp9 (subunit $c$ ) together with one copy of Atp6 (subunit $a$ ). Additionally, the yeast $\mathrm{F}_{\mathrm{o}}$ part of $\mathrm{F}_{1} \mathrm{~F}_{\mathrm{o}}$-ATP synthase contains Atp17 
and Atp8, both essential for proper assembly and function (Devenish et al., 2000). The $\mathrm{F}_{\mathrm{o}}$ part couples proton movement down the electrochemical gradient to the rotation of the Atp9 ring (Weber and Senior, 2003). The central stalk, built from $\operatorname{Atp} 3\left(\mathrm{~F}_{1} \gamma\right), \operatorname{Atp} 15\left(\mathrm{~F}_{1} \varepsilon\right)$ and $\operatorname{Atp} 16\left(\mathrm{~F}_{1} \delta\right)$, transmits these rotational movements to the catalytically active region by elastic power transmission (Noji et al., 1997). The peripheral (or lateral) stalk interacts with both $\mathrm{F}_{1}$ and $\mathrm{F}_{\mathrm{o}}$ and keeps the catalytically active domain static, relative to the rotary element of the enzyme. Atp4 (subunit b), Atp5 (OSCP), Atp7 (subunit d) and Atp14 (subunit h) form the peripheral stalk (Walker and Dikson 2006).

Table 1.2 Subunits of the S. cerevisiae ATP synthase

\begin{tabular}{|c|c|c|c|c|c|}
\hline Subunit & Synonym & Gene & Genome & Module & Essentiality \\
\hline$\alpha$ & Atp1 & ATP1 & $\mathrm{N}$ & $\mathrm{F}_{1}$, catalytic head & Essential \\
\hline$\beta$ & Atp2 & ATP2 & $\mathrm{N}$ & $\mathrm{F}_{1}$, catalytic head & Essential \\
\hline$\gamma$ & Atp3 & ATP3 & $\mathrm{N}$ & $\mathrm{F}_{1}$, central stalk & Essential \\
\hline$\delta$ & Atp16 & ATP16 & $\mathrm{N}$ & $\mathrm{F}_{1}$, central stalk & Essential \\
\hline$\varepsilon$ & Atp15 & ATP15 & $\mathrm{N}$ & $\mathrm{F}_{1}$, central stalk & Essential \\
\hline$b$ & Atp4 & ATP4 & $\mathrm{N}$ & $\mathrm{F}_{\mathrm{o}}$, lateral stalk & Essential \\
\hline$d$ & Atp7 & ATP7 & $\mathrm{N}$ & $\mathrm{F}_{\mathrm{o}}$, lateral stalk & Essential \\
\hline$h$ & Atp14 & ATP14 & $\mathrm{N}$ & $\mathrm{F}_{\mathrm{o}}$, lateral stalk & Essential \\
\hline OSCP & Atp5 & ATP5 & $\mathrm{N}$ & $\mathrm{F}_{\mathrm{o}}$, lateral stalk & Essential \\
\hline$f$ & Atp17 & ATP17 & $\mathrm{N}$ & $\mathrm{F}_{\mathrm{o}}$, lateral stalk & Essential \\
\hline$a$ & Atp6 & ATP6 & M & $\mathrm{F}_{\mathrm{o}}$, rotor & Essential \\
\hline 8 & Atp8 & ATP8 & M & $\mathrm{F}_{\mathrm{o}}$, rotor & Essential \\
\hline$c$ & Atp9 & ATP9 & M & $\mathrm{F}_{\mathrm{o}}$, rotor & Essential \\
\hline$j / i$ & Atp18 & ATP18 & $\mathrm{N}$ & Dimer-specific & Dispensable \\
\hline$e$ & Atp21 & ATP21/TIM11 & $\mathrm{N}$ & Dimer-specific & Dispensable \\
\hline$g$ & Atp20 & ATP20 & $\mathrm{N}$ & Dimer-specific & Dispensable \\
\hline$k$ & Atp19 & ATP19 & $\mathrm{N}$ & Dimer-specific & Dispensable \\
\hline Inh1 & Inh1 & INH1 & $\mathrm{N}$ & Regulatory & Dispensable \\
\hline Stf1 & Stf1 & STF1 & $\mathrm{N}$ & Regulatory & Dispensable \\
\hline Stf2 & Stf2 & STF2 & $\mathrm{N}$ & Regulatory & Dispensable \\
\hline
\end{tabular}

N, nuclear genome; M, mitochondrial genome. Modified from Wittig and Schägger, 2008 and Rühle and Leister, 2015.

The complete $\mathrm{F}_{1} \mathrm{~F}_{\mathrm{o}}$-ATP synthase is known to form dimers and oligomers (Arnold et al., 1998). Such oligomerization is needed for maintaining the cristae structure and local increase in membrane potential. Recently yeast dimer specific subunits were found: Atp18 (subunit i/j), Atp19 (subunit k), Atp20 (subunit g) and Atp21 (subunit e). Loss of these subunits does not affect enzyme activity but prevents it dimerization. 


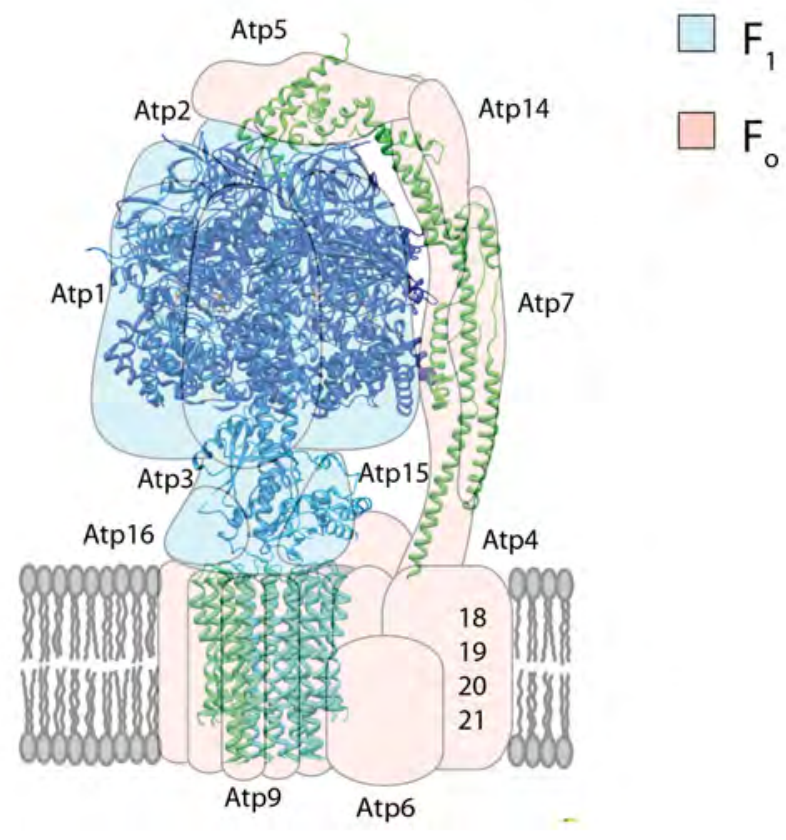

Figure 1.6 Structure of the $S$. cerevisiae $\mathbf{F}_{1} \mathbf{F}_{\mathrm{o}}$-ATP synthase complex. The catalytical "head" and central stalk form the $\mathrm{F}_{1}$ subunit. $10 c$ subunits form an oligomeric ring, through which protons traverse the membrane. Subunit a remains membrane-bound and forms contacts with the lateral stalk to prevent rotation of the catalytic domain. The OSCP subunit links the peripheral stalk to the $\mathrm{F}_{1}$. PDB accession number $4 \mathrm{~b} 2 \mathrm{q}$, Davies et al., 2012.

\subsubsection{Mechanism of ATP synthesis by $F_{1} F_{0}$-ATP synthase}

Energy produced by proton translocation across the membrane is used by the $\mathrm{F}_{1} \mathrm{~F}_{\mathrm{o}}$-ATP synthase to catalyze ATP production from ADP and inorganic phosphate (Weber and Senior, 2003). It is important to understand that proton translocation is performed by the membrane rotor, whereas ATP synthesis happens on the catalytic head. The central stalk directly links these two modules to each other and transmits the energy between them by elastic power transmission. The peripheral (lateral) stalk keeps the catalytic head static, which is absolutely necessary for the coupling of proton translocation and ATP production.

Atp6 interacts directly with Atp9 subunits in the rotor. The C-terminal domain of each Atp9 subunit contains negatively charged carboxy groups of either Gln or Asn residues exposed on the outside of the ring. Protons enter a channel formed by Atp6 and Atp9 from the IMS side and neutralize the negative charge of the abovementioned residues. Once the residue is neutralized, it changes its conformation in order to move more to the hydrophobic lipid environment that induces rotation of the Atp9 ring. This translocation brings another negatively charged residue to the channel to keep the rotation going. While moving around the rotational axis, the neutralized residue reaches another site on the Atp6/Atp9 interface which has a local environment that promotes its reionization and subsequent proton release from the 
IMS side of the channel (Nakamoto et al., 2008; Symersky et al., 2012). Movement of the rotor, in turn, induces conformational changes in the Atp3 subunit which passes this structural transformation to the Atp1/Atp2 dimers. The asymmetry of Atp3 promotes each of the three Atp2 subunits within a hexamer to adopt different conformations that differentiate their nucleotide-binding and catalytic properties. Therefore, conformational changes in Atp3 driven by Atp9 ring rotation drive the catalytic cycle of ATP synthesis (Senior et al., 2002).

\subsubsection{Biogenesis of mitochondrial-encoded $\mathrm{F}_{1} \mathrm{~F}_{\mathrm{o}}$-ATP synthase subunits}

Biogenesis of Atp9. Translation of ATP9 mRNA, as well as its stability, depends on Aep1 (acts as a translation activator), Aep2 (regulates either mRNA stability or its translation) and Atp25 (has a dual role in translation of Atp9, as well as in ATP synthase assembly) (Ackerman et al., 1991; Finnegan et al., 1991; Finnegan et al., 1995; Payne et al., 1993; Ellis et al., 1999; Zeng et al., 2008). The dual role of Atp25 can be explained by its unusual biogenesis. After synthesis, Atp25 is cleaved in two fragments that both get imported into mitochondria and have distinct functions. The C-terminal fragment of $25 \mathrm{kDa}$ stabilizes ATP9 mRNA and promotes its translation. The N-terminal part of Atp25 promotes Atp9 oligomerization and, therefore, ATP synthase assembly (Zeng et al., 2008). Oligomerization of Atp9 is an important step in complex $\mathrm{V}$ biogenesis and happens independently from all other subunits. Interestingly, Atp9 membrane insertion is Oxa1-independent, implying that either Atp9 does not need a membrane channel for insertion, or most likely the required protein is not yet identified. However, Oxa1 is required for Atp9 ring assembly with Atp6 and Atp8 (Jia et al., 2007).

Biogenesis of Atp6 and Atp8. Atp6 and Atp8 are encoded on a bicistronic mRNA but it seems that they are translated independently. Atp22 was characterized as an Atp6-specific translational activator (Helfenbein et al., 2003; Zeng et al., 2007a). In the absence of ATP22, Atp6 is no longer produced, however, Atp8 is still translated. Atp 8 translation is activated by another, most likely not yet identified protein. Overall, much more is known about early Atp6 biogenesis, whereas our knowledge about Atp8 is significantly limited (see Fig. 1.7).

An interesting mechanism exists to coordinate expression of Atp6 and Atp8 with nuclear-encoded ATP synthase subunits. It was shown that mutants devoid of a fully-assembled $F_{1}$ fail to express Atp6 and Atp8, but translation of both proteins could be rescued by the overexpression of Atp22 (Rak and Tzagoloff, 2009). Recently, a novel protein was characterized to be involved in the regulation of $\mathrm{F}_{1}$ dependent Atp6/Atp8 expression, called Smt1 (Rak et al., 2016). It was shown that in smt1 $\Delta$, Atp6 and Atp8 translation is no longer dependent on a fully-assembled $\mathrm{F}_{1}$. Smt1 is present in ATP6/ATP 8 mRNA containing complexes and probably interacts with the ATP $6 / A T P 8$ mRNA. The authors proposed that Smt1 acts as an 
inhibitor of Atp6 and Atp8 translation by competing with Atp22 for mRNA binding. The fully assembled $\mathrm{F}_{1}$ must therefore interact with Smt1 to trigger ATP6/ATP8 mRNA release and to enable Atp22 activation of Atp6 translation. Thus, in the absence of Smt1, ATP6/ATP8 mRNA is not repressed and $\mathrm{F}_{1}$-dependent activation of translation is not required.

Another observation that linked Atp6 expression to that of the nuclear-encoded structural subunits came from the analysis of mitochondria that were depleted of Atp14 (Goyon et al., 2008). Atp14 is a small subunit of the peripheral stalk and is not directly associated with the IMM. Cells, in which Atp14 levels were reduced to $3 \%$, showed a significant reduction of Atp6, whereas Atp8 and Atp9 were not affected. It was shown that Atp6 synthesis, as well as stability, were severely affected. Moreover, mutant mitochondria showed a significant reduction of mature complex $\mathrm{V}$ and accumulation of the unassembled $F_{1}$ module. This data indicates that not only $\mathrm{F}_{1}$, but also the peripheral stalk, influences translation of mitochondrial-encoded Atp6.

S. cerevisiae Atp6 is synthesized with a 10 amino acid N-terminal extension that is processed after the protein is inserted into the inner mitochondrial membrane and the N-terminus is exposed to the IMS (Michon et al., 1988). The cleavage is performed by Atp23, a highly conserved protease of the IMS, which is peripherally associated to the inner mitochondrial membrane (Osman et al., 2007; Zeng et al., 2007c). It was shown that Atp23 is imported and folded by Mia40, however, the Cys residues of Atp23 are dispensable for its import (Weckbecker et al., 2012). Deletion of Atp23 results in accumulation of unprocessed Atp6 and absence of a mature complex $\mathrm{V}$.

Atp23 is a metalloprotease with the catalytic center formed by Histidine residues. Surprisingly, the catalytic activity of Atp23 and therefore the processing of Atp6 is not required for ATP synthase biogenesis (Osman et al., 2007; Zeng et al., 2007c). However, the protease is essential, implying that apart from Atp6 processing it is required for Atp6 assembly. This assumption is supported by the observation that Atp6 devoid of a leader peptide assembles less efficiently into complex V compared to wild type Atp6 (Zeng et al., 2007b). Though the steady-state amounts of leaderless Atp6 are not affected, amounts of mature ATP synthase are reduced almost two-fold. Thus, the leader peptide carries information required for Atp6 assembly. Therefore, it is tempting to speculate that this peptide is required for Atp6-Atp23 interaction, and is needed for Atp23 to facilitate Atp6 assembly.

Atp10 is another protein involved in the early biogenesis of the $\mathrm{F}_{1} \mathrm{~F}_{\mathrm{o}}$-ATP synthase (Ackerman and Tzagoloff, 1990a; Tzagoloff et al., 2004). It is a 30-kDa inner membrane protein that does not co-fractionate with mature complex V and, therefore, is not a structural subunit. Deletion of ATP10 does not affect Atp6 translation, but significantly increases its turnover. Atp10 can be cross-linked to Atp6 (Tzagoloff 


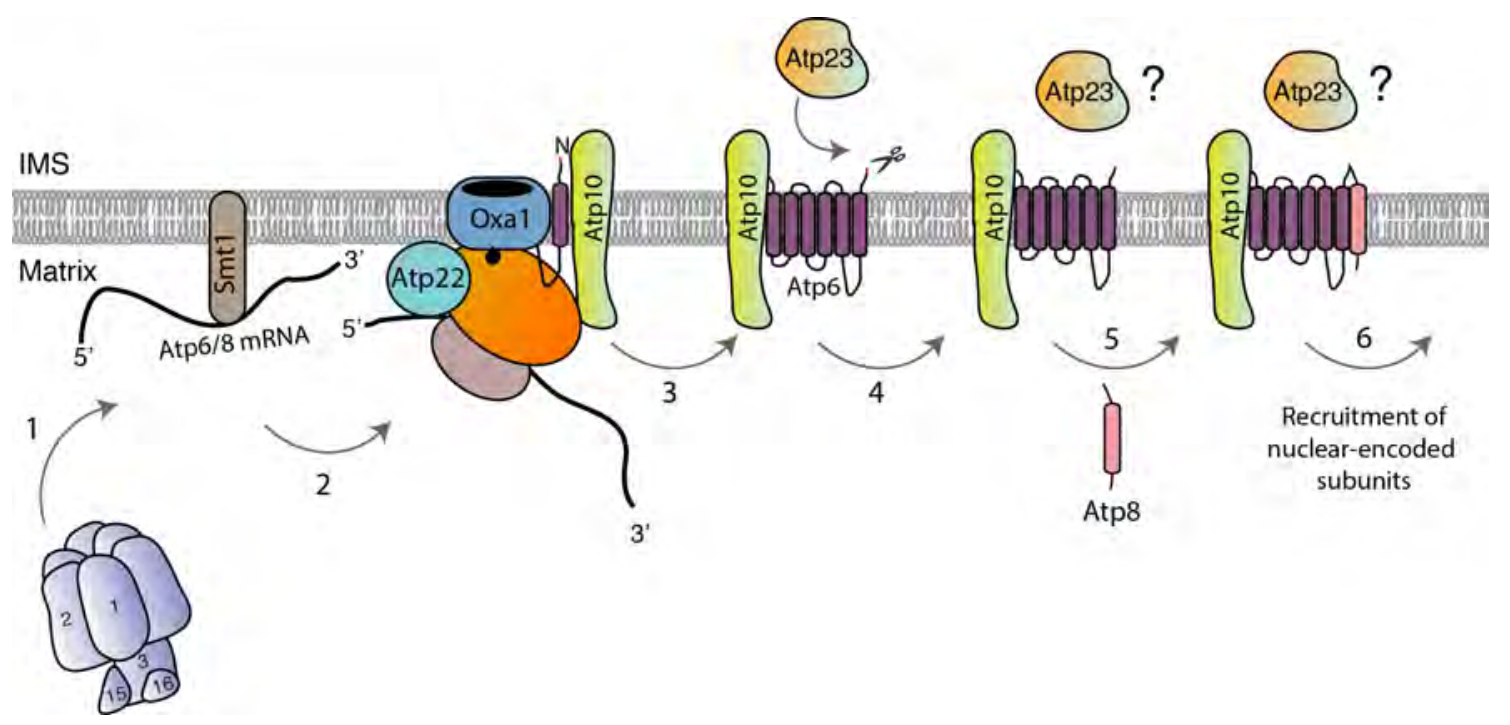

Figure 1.7 Schematic representation of early Atp6/Atp8 biogenesis. Atp6 and Atp8 are encoded on a bicistronic mRNA. Smt1 binds ATP6/ATP8 transcript to repress its translation. 1. Assembled $F_{1}$ module presumably interacts with Smt1 and triggers mRNA release. 2. Atp22 interacts with the 5' UTR of the released mRNA to activate its translation. Pre-Atp6 is inserted into the IMM co-translationally by Oxa1 and associates with Atp10 for stabilization. 3. Atp23 cleaves the N-terminal signal sequence of Atp6. It is not known whether it associates stably with Atp10. 4. The processed Atp6 remains associated with Atp10 and is already competent to assemble with Atp8. It is likely that Atp23 remains associated with this intermediate, but experimental evidence for this is missing. 5. Atp8 assembles with Atp6 and Atp10 to form an Atp6/Atp8 module, capable of interaction with the nuclear-encoded subunits (6.). IMM, inner mitochondrial membrane; IMS, inter membrane space; UTR, untranslated region.

et al., 2004), suggesting that this direct interaction is required for Atp6 stabilization. Interestingly, apart from its chaperone function, Atp10 might act as an $\mathrm{F}_{\mathrm{o}}$ assembly factor. Overexpression of Atp23 suppresses the Atp10 deletion phenotype, most probably because Atp10 and Atp23 have partly overlapping functions in Atp6 stabilization and assembly (Zeng et al., 2007c). However, a physical interaction between Atp10 and Atp23 has not yet been shown. Recently, it was reported that Atp10 remains associated with Atp6 as long as it binds Atp8 and peripheral stalk subunits to form a larger assembly intermediate (Rak et al., 2011).

\subsubsection{Assembly of the yeast $\mathrm{F}_{1} \mathrm{~F}_{\mathrm{o}}$-ATP synthase}

Yeast mitochondrial $\mathrm{F}_{1} \mathrm{~F}_{\mathrm{o}}$-ATP synthase assembles in a modular way from preassembled intermediates with $\mathrm{F}_{1}$ assembling independently from $\mathrm{F}_{\mathrm{o}}$ (Schatz, 1968; Tzagoloff, 1969; Rak et al., 2011). According to Rak et al., 2011, two major assembly intermediates are formed from both nuclear and mitochondrial encoded subunits and are combined together by a not yet clearly defined mechanism (Fig. 1.8). 




Figure 1.8 Model of $\mathbf{F}_{\mathbf{1}} \mathbf{F}_{\mathbf{o}}$-ATP synthase assembly. Assembly of $\mathrm{F}_{1} \mathrm{~F}_{\mathrm{o}}$-ATP synthase starts from Atp9 oligomerization. The Atp9 ring interacts with a preassembled $\mathrm{F}_{1}$ part to form a distinct assembly intermediate. On the other hand, Atp6 and Atp8, together with Atp10, associate with the peripheral stalk to form the Atp6/Atp8 module. It is currently unknown how Atp5 is assembled, however, it is thought not to be a part of the Atp6/Atp8 module. Atp9 ring/ $\mathrm{F}_{1}$ joins with the Atp6/Atp8 assembly module in the last assembly step to form the mature enzyme. It is not known how Atp10 is released from the assembly intermediate. IMS, inter membrane space; UTR, untranslated region. Adapted from Rak et al., 2011. 
The soluble $\mathrm{F}_{1}$ module is formed independently from all other intermediates (Schatz, 1968; Tzagoloff, 1969). Atp1 and Atp2 subunits oligomerize in a hexamer with the help of Atp12 and Atp11 chaperones, respectively (Ackerman and Tzagoloff, 1990b; Ackerman and Tzagoloff, 2002). In the absence of either protein, Atp1 and Atp2 form insoluble aggregates in the mitochondrial matrix and fail to assemble.

Both chaperones bind their client proteins to form Atp1-Atp12 and Atp2-Atp11 heterodimers in order to prevent formation of Atp1-Atp1 and Atp2-Atp2 homodimers (Wang, 2000; Wang et al., 2000). The reported 3D structure of Atp12 from Paracoccus denitrificants revealed that the C-terminus of Atp12 is similar to the coiled-coil tail of Atp3. Therefore, binding of Atp3 might trigger Atp12 release and promote Atp1-Atp2 interaction (Ludlam, 2009). Two more proteins are required for efficient $\mathrm{F}_{1}$ assembly under heat stress conditions - Fmc1 and Hsp90 (Lefebvre-Legendre, 2001; Francis and Thorsness, 2011). Fmc1 is most likely an Atp12-specific chaperone, as Fmc1 deletion can be compensated by overexpression of Atp12 (Lefebvre-Legendre, 2001). Once assembled, $\mathrm{F}_{1}$ binds to the Atp9 ring in order to form the first large assembly intermediate (Rak et al., 2011).

Another intermediate is formed by Atp6/Atp8 and components of the peripheral (lateral) stalk. According to Rak et al., 2011, Atp6 interacts with Atp8 and Atp10, but not Atp23, in order to form an Atp6/Atp8 module. This module further associates with Atp4, Atp7 and Atp14 lateral stalk subunits. Assembly of Atp5 is not clearly understood and likely happens independently.

According to the described assembly model, the pore-forming subunits, Atp9 and Atp6, assemble only in the last step after the nuclear-encoded subunits are already positioned. Such a mechanism ensures that when the Atp9 ring interacts with Atp6, the proton translocation across the membrane will be definitely coupled to ATP synthesis and a futile membrane potential dissipation will be avoided. However, it is not known whether additional factors are involved in preventing a premature Atp6/Atp9 interaction.

The fully assembled $\mathrm{F}_{1} \mathrm{~F}_{\mathrm{o}}$-ATP synthase dimerizes via Atp4 transmembrane helixes (Davies et al., 2012; Davies et al., 2014). Moreover, dimerization requires the incorporation of Atp20 and Atp21 (Davies et al., 2012), which is facilitated by subunits Atp18 and Atp19 (Wagner et al., 2010). Moreover, complex V forms higher oligomeric structures, but little is known about the mechanism and regulation of their formation (Strauss et al., 2008; Davies et al., 2012).

\subsubsection{Role of the INA complex in $\mathrm{F}_{1} \mathrm{~F}_{\mathrm{o}}$-ATP synthase assembly}

Recently, a novel Inner Membrane Assembly Complex (INAC) was identified that facilitates $\mathrm{F}_{1} \mathrm{~F}_{\mathrm{o}}$-ATP synthase assembly (Lytovchenko et al., 2014). The complex consists of two inner membrane proteins, Ina22 and Ina17. Ina22 and Ina17 are highly conserved in the fungal kingdom, but homologs have so far not been identified 
in higher eukaryotes. Both proteins interact with each other via coiled-coil domains, located in the mitochondrial IMS. Interestingly, Ina22 becomes highly unstable upon INA17 deletion, implying that Ina17 might fulfill a chaperone-like function.

Absence of either protein leads to a respiration defect, which is more pronounced at high temperatures. Deletion of the INA complex components reduces the amounts of mature $\mathrm{F}_{1} \mathrm{~F}_{\mathrm{o}}$-ATP synthase and leads to a significant accumulation of unassembled $F_{1}$. As the steady-state levels of individual structural subunits of complex $\mathrm{V}$ were not affected in ina mutants, the authors assumed that the assembly process is impaired. However, INAC is obviously not essential for $\mathrm{F}_{1} \mathrm{~F}_{\mathrm{o}}$-ATP synthase biogenesis as the mature complex is still present in mutant mitochondria, albeit at lower levels. Importantly, a detailed analysis of respiratory chain complexes in ina mutants did not reveal their deficiency.

Quantitative mass spectrometry analysis of Ina $22^{\text {ProtA }}$-containing protein complexes revealed that INAC physically associates with the nuclear-encoded $\mathrm{F}_{1} \mathrm{~F}_{\mathrm{o}}$-ATP synthase subunits and with the Cbp3, Cbp6 and Cbp4 - proteins involved in biogenesis of cytochrome $b$. This finding pointed not only to a direct association of INAC with ATP synthase, but also to a possible role of the INA complex in biogenesis of complex III. However, the latter was not investigated further.

Surprisingly, when incorporation of nuclear-encoded radiolabeled $\mathrm{F}_{1} \mathrm{~F}_{\mathrm{o}}$-ATP synthase subunits into the mature enzyme was analyzed, it appeared that assembly of the peripheral stalk was affected the most, whereas assembly of the $\mathrm{F}_{1}$ components was only mildly reduced. This observation, together with other data, led to the conclusion that INAC facilitates assembly of the peripheral stalk. However, the exact mechanism of how INAC facilitates peripheral stalk biogenesis in context of Atp6/Atp8 module assembly and formation of Atp9 ring/ $F_{1}$ intermediate was not investigated in detail. Moreover, it is not clear and therefore tempting to speculate whether INAC could possibly link assembly of nuclear- and mitochondrial-encoded complex V subunits. 


\subsection{Aim of this study}

The $\mathrm{F}_{1} \mathrm{~F}_{\mathrm{o}}$-ATP synthase is one of the most fascinating molecular machines present in the cell. It has been preserved by virtually all cells in the course of evolution. Although structural differences exist among $\mathrm{F}_{1} \mathrm{~F}_{\mathrm{o}}$-ATP synthases of different origin, the mechanism of ATP synthesis is conserved.

During the past decades a lot has been done to unravel the structural composition of mitochondrial $\mathrm{F}_{1} \mathrm{~F}_{\mathrm{o}}$-ATP synthases and the mechanism of ATP production. However, we start to understand only now how these complexes of dual genetic origin come into life. Still, much information is missing about how the individual assembly intermediates of the $\mathrm{F}_{1} \mathrm{~F}_{\mathrm{o}}$-ATP synthase are formed and linked together. Moreover, very little is known about the regulatory mechanisms that keep complex $\mathrm{V}$ biogenesis under control.

Recently, the INA complex was discovered that facilitates assembly of the peripheral stalk of the $\mathrm{F}_{1} \mathrm{~F}_{\mathrm{o}}$-ATP synthase. The physical interaction between INAC and nuclear-encoded subunits of complex $\mathrm{V}$ was characterized, however, it was not put into context of the whole $\mathrm{F}_{1} \mathrm{~F}_{\mathrm{o}}$-ATP synthase assembly pathway. Therefore, a possible interaction of the INA complex with other proteins of the analyzed $\mathrm{F}_{1} \mathrm{~F}_{\mathrm{o}^{-}}$ ATP synthase assembly machinery is investigated in this study. Moreover, the order of sequential steps in complex $\mathrm{V}$ assembly is examined in more detail to create a comprehensive picture of the $\mathrm{F}_{1} \mathrm{~F}_{\mathrm{o}}$-synthase biogenesis. Most importantly, it is addressed whether the INA complex participates in the biogenesis of Atp6, Atp8 and Atp9 and liaises nuclear and mitochondrial encoded subunits during their assembly.

The current model of $\mathrm{F}_{1} \mathrm{~F}_{\mathrm{o}}$-ATP synthase assembly postulates the formation of two distinct assembly intermediates, each containing one half of the $\mathrm{F}_{\mathrm{o}}$ membrane proton channel. Although formation of this channel is, without a doubt, the most crucial step in complex $\mathrm{V}$ biogenesis, surprisingly little is known about the machinery that mediates it. Therefore, I set out to unravel the role of INAC in the final assembly step, namely the connection of the above mentioned intermediates and formation of the membrane proton path.

Finally, a possible function of INAC in the biogenesis of complex III will be investigated, as well a possible connection for complex V and III assembly. 


\section{Results}

\subsection{Deletion of INA complex subunits affects biogenesis of mitochondrial-encoded $F_{1} F_{0}-A T P$ synthase components}

\subsubsection{Translation of Atp6 and Atp8 is upregulated in ina mutants, whereas Atp9 is destabilized}

Loss of the INA complex, consisting of Ina17 and Ina22, leads to impaired respiration and therefore slower yeast growth on non-fermentable carbon sources (Lytovchenko et al., 2014). The observed phenotype is caused by an impaired assembly of the main energy-producing protein complex in the cell, the $\mathrm{F}_{1} \mathrm{~F}_{\mathrm{o}}$-ATP synthase. Whereas steady-state levels of the nuclear-encoded subunits of the $\mathrm{F}_{1} \mathrm{~F}_{\mathrm{o}}$-ATP synthase are not affected in ina mutants, their assembly rate is severely reduced, resulting in the accumulation of free unassembled $\mathrm{F}_{1}$-module. Interestingly, assembly of the peripheral stalk in ina mutant mitochondria was affected the most. According to a recently proposed $\mathrm{F}_{1} \mathrm{~F}_{\mathrm{o}}$-ATP synthase assembly model, the nuclear-encoded peripheral stalk associates with the mitochondrial-encoded Atp6/Atp8 module in order to form a single assembly intermediate (Rak et al., 2011). Therefore, we asked whether impaired peripheral stalk assembly in ina mutants affects Atp6/Atp8 biogenesis or, alternatively, if impaired Atp6/Atp8 biogenesis is a prerequisite for the observed $\mathrm{F}_{1} \mathrm{~F}_{\mathrm{o}}$-ATP synthase assembly defect. To address this, we performed pulse-labeling of mitochondrial-encoded proteins with $\left[{ }^{35} \mathrm{~S}\right]$ methionine and analyzed translation products by Urea SDS-PAGE and digital autoradiography. Interestingly, a drastic increase of Atp6 amounts, compared to wild type, was observed in ina22 $\Delta$ and ina17 4 , whereas translation of other subunits (e. g. Cox1) was not affected (Fig. 2.1A). To distinguish whether the increased Atp6 abundance in the cell was caused by an increased translation rate or increased stability, we chased newly-translated mitochondrial gene products in wild type and mutant strains. Clearly, Atp6 stability in ina mutants was not increased sufficient enough to explain its significantly higher abundance (Fig. 2.1B). Therefore it was concluded that translation of Atp6 is upregulated in ina22 $\Delta$ and ina17 $\Delta$. Next we asked whether the translation of two other mitochondrial-encoded subunits of $\mathrm{F}_{1} \mathrm{~F}_{\mathrm{o}}$-ATP synthase, namely Atp 8 and Atp9, is also affected in ina mutants. Therefore, mitochondrial translation products 


\section{A}
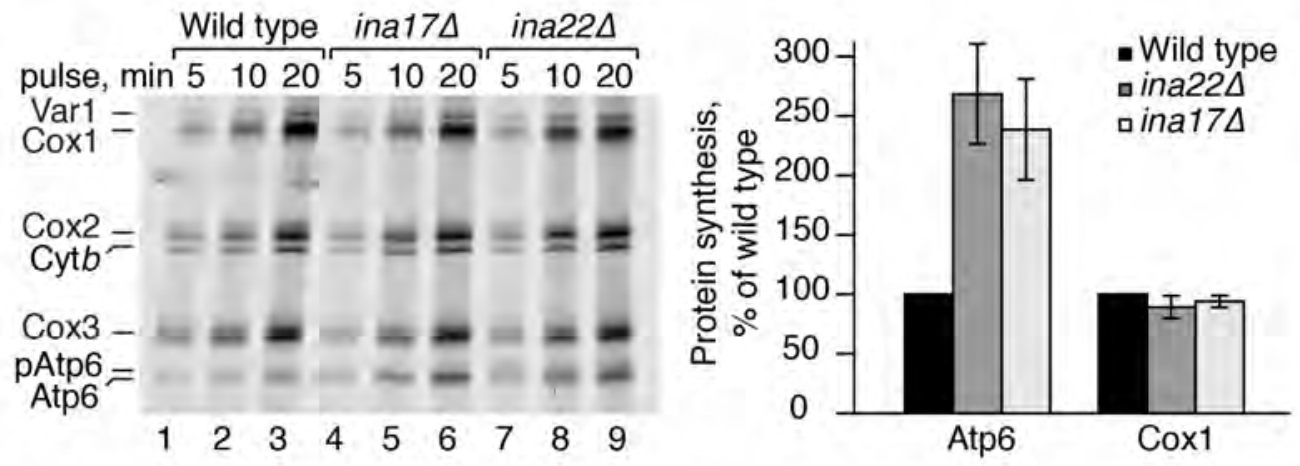

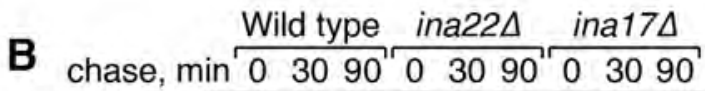
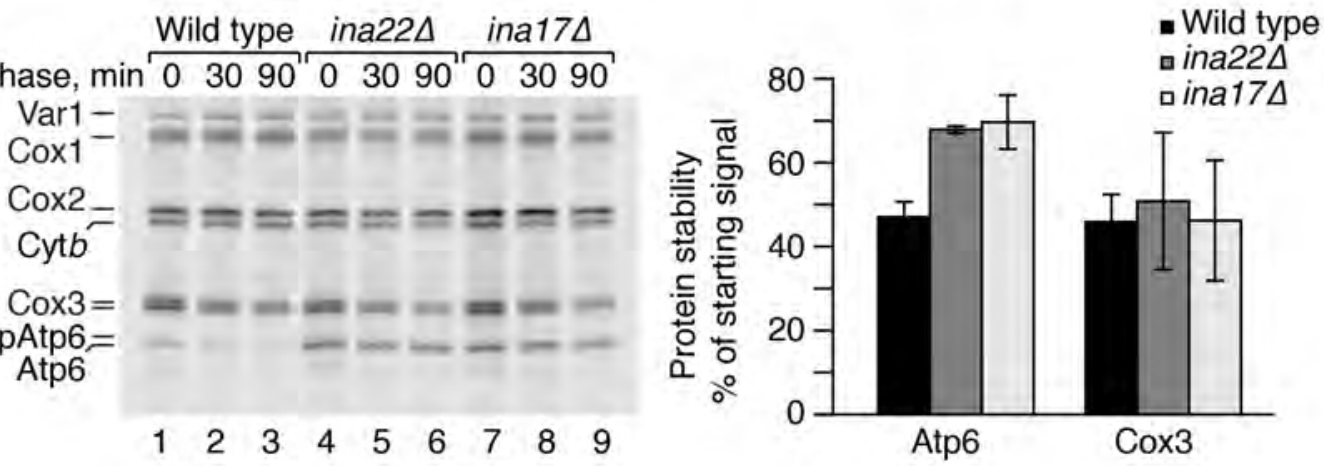

Figure 2.1 Atp6 translation is upregulated in ina22 $\Delta$ and ina17 $\Delta$. A. Mitochondrialencoded proteins were radiolabeled in vivo and analyzed by Urea SDS-PAGE and digital autoradiography. Atp6 and Cox1 signals after a 20 min pulse were normalized to Cox3 signal and protein amounts in mutant mitochondria were quantified (wild type $=100 \%) .(\mathrm{n}=3, \pm \mathrm{SEM})$. B. Mitochondrial-encoded proteins were radiolabeled in vivo for $10 \mathrm{~min}$. Labeling was stopped with an excess of cold methionine, samples were incubated for an additional 30 or 90 min and analyzed as in A. Atp6 and Cox3 signals after a 90 min chase were quantified as the percentage of their starting signals. $(\mathrm{n}=3, \pm \mathrm{SEM})$.

were labeled in vivo as in Figure 2.1A and analyzed by Tris-Tricine SDS-PAGE in order to separate Atp8 and Atp9. Clearly, Atp8 amounts were drastically increased in mutant cells (Fig. 2.2A), similar to the increased abundance of Atp6. However, we did not detect any significant changes for Atp9 or other mitochondrial-encoded components of the respiratory chain. Surprisingly, when a similar analysis of mitochondrial translation was performed in organello, a significant decrease in Atp9 translation as well as amounts of formed Atp9 ring was detected in ina mutants (Fig. 2.2B). Most likely, Atp9 stability depends on imported nuclear-encoded proteins with a high turn-over rate. In purified mitochondria these factors are not delivered to the organelle and this causes a decrease of Atp9 amounts.

To summarize, it was established that Atp6 and Atp8 are significantly upregulated in ina mutants, whereas Atp9 production is not changed. However, we speculate that Atp9 stability is decreased in ina mutants. 
A

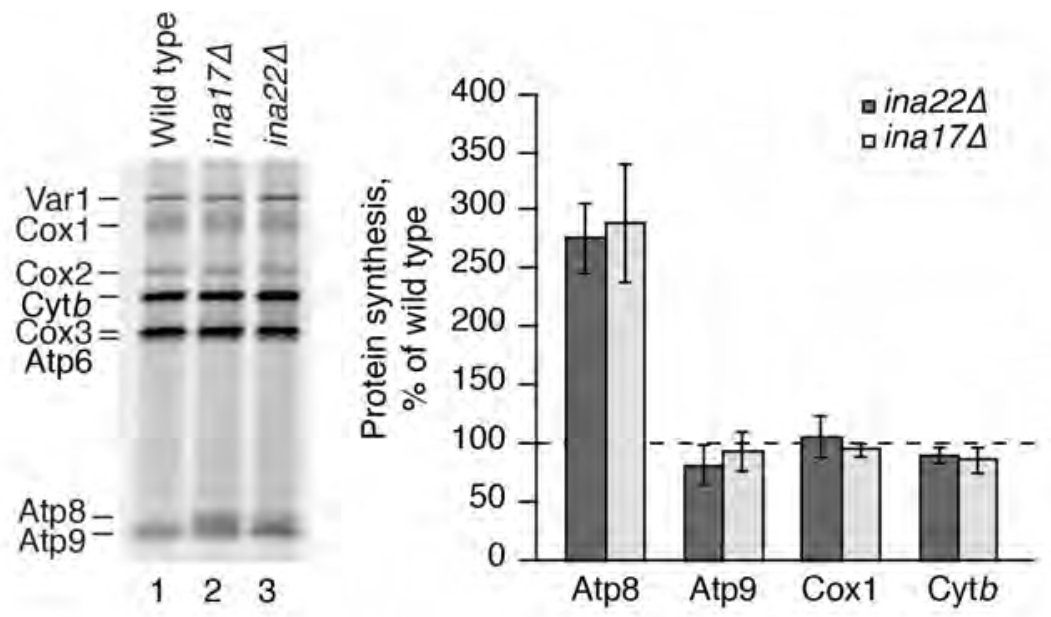

B

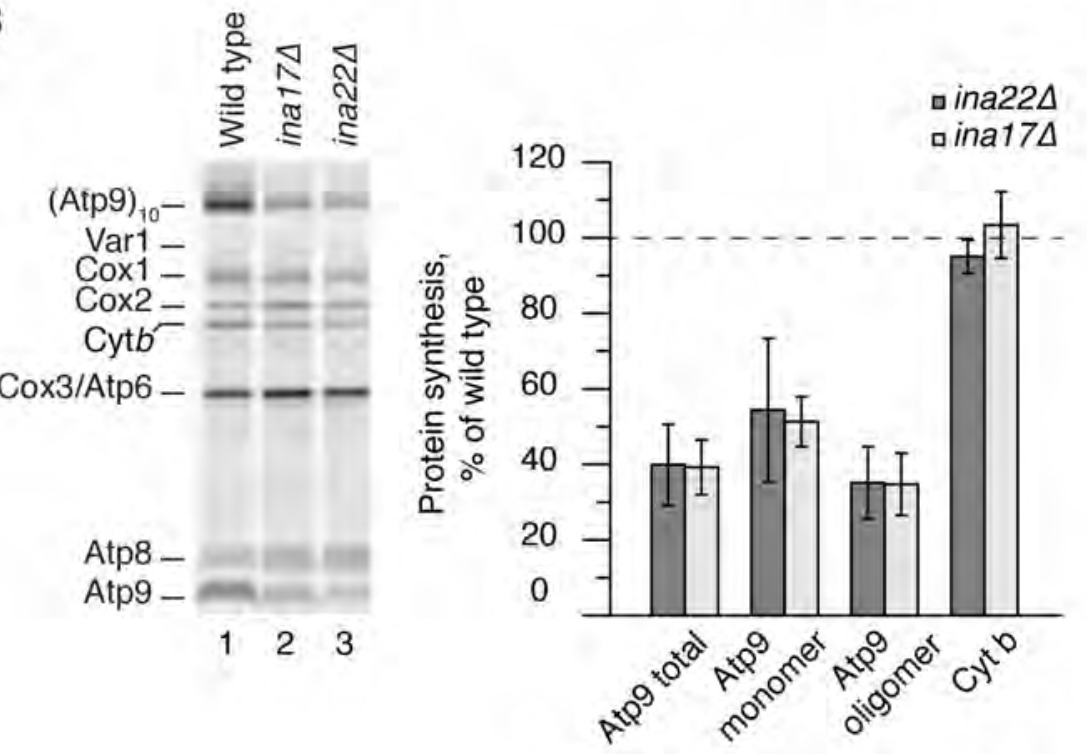

Figure 2.2 Atp8 and Atp9 levels are changed in ina mutants. A. Mitochondrial translation products were radiolabeled in vivo and analyzed by Tris-Tricine PAGE and autoradiography. Signals in ina22 $\Delta$ and ina17 $\Delta$ were normalized to Var1 and quantified as the percentage of wild type. $(n=5, \pm S E M)$. B. Mitochondrial translation products were radiolabeled in organello for $20 \mathrm{~min}$ and analyzed by SDS-PAGE and autoradiography. After normalization to Cox1, Atp9 and cytochrome b (Cytb) signals in ina22 $\Delta$ and ina17 $\Delta$ mitochondria were quantified as the percentage of wild type. $(\mathrm{n}=3, \pm \mathrm{SEM})$.

\subsubsection{INA complex does not associate with mitochondrial ribosomes}

Translation of mitochondrial-encoded proteins in yeast is under the strict control of translational regulators that associate with mitochondrial ribosomes in order to coordinate expression of mitochondrial and nuclear-encoded subunits of the respiratory chain (Richter-Dennerlein et al., 2015). Taking into account the significant changes in expression of mitochondrial-encoded $\mathrm{F}_{1} \mathrm{~F}_{\mathrm{o}}$-ATP synthase components in ina mutants, we did not exclude the possibility that INAC associates with mitochondrial ribosomes in order to regulate expression of mitochondrial-encoded proteins. 
Therefore, we centrifuged mitochondrial lysates through a sucrose cushion in order to pellet mitochondrial ribosomes and the proteins associated with them, and analyzed the ribosome-containing pellet and supernatant fraction by SDS-PAGE and Western blotting. As expected, Cbp3, a cytochrome $b$ translational activator, was sedimented with ribosomes (see Mrpl4 and Mrpl36) in a salt-dependent manner (Gruschke et al., 2011) (Fig. 2.3). Importantly, neither Ina17 nor Ina22 were recovered from the ribosome-containing pellet, implying that the INA complex does not associate with mitochondrial ribosomes and therefore upregulation of Atp6 and Atp8 translation might be an indirect effect of INAC knockout.

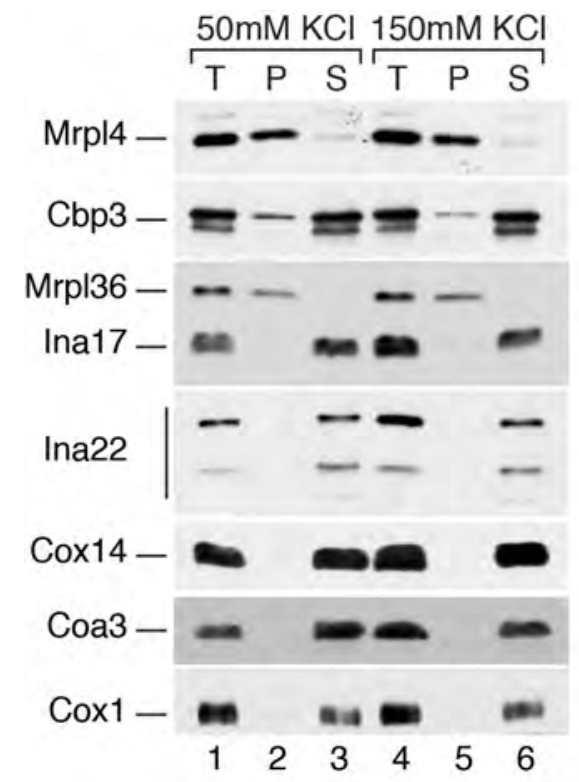

Figure 2.3 INA complex does not associate with mitochondrial ribosomes. Wild type mitochondria were lysed in a buffer containing 1\% Triton-X100 and either 50 or 150 $\mathrm{mM} \mathrm{KCl}$ and centrifuged through a sucrose cushion. Total $(\mathrm{T})$, pellet $(\mathrm{P})$ and supernatant $(\mathrm{S})$ fractions were precipitated with $12 \%$ TCA and analyzed by SDS-PAGE and Western blotting with the indicated antibodies.

\subsubsection{Atp6 processing is impaired in ina22 $\Delta$}

In yeast mitochondria, Atp6 is synthesized as a precursor with a 10 amino acid N-terminal extension, which is processed by Atp23 on the intermembrane space side of the inner mitochondrial membrane (Osman et al., 2007; Zeng et al., 2007c). During the initial analysis of Atp6 translation in ina mutants, we noticed the accumulation of an additional radiolabeled product migrating above Atp6 in ina22 $\Delta$ (Fig. 2.1A, lanes 7-9). To investigate this further, a radiolabeling of mitochondrialencoded proteins was performed in wild type and mutant mitochondria, followed by a separation of precursor and mature form of Atp6 by Urea SDS-PAGE. While Atp6 processing appeared unaffected in ina17 1 , a significant amount of preAtp6 accumulated upon INA22 deletion (Fig. 2.4A, lanes 7-9). To exclude that Atp23 levels 

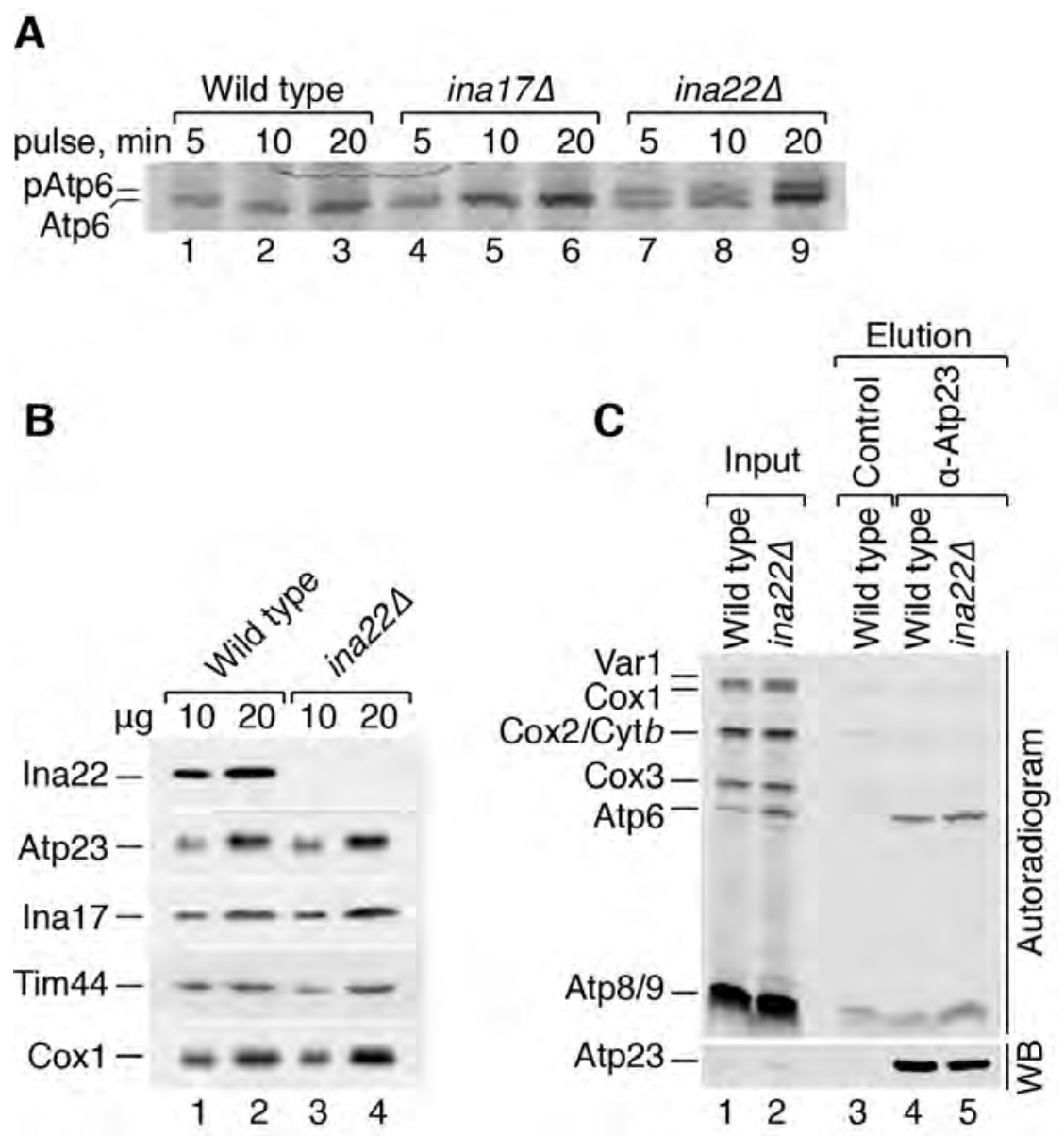

Figure 2.4 Atp6 processing is affected in ina22 $\Delta$. A. Mitochondrial-encoded proteins were radiolabeled in vivo and analyzed by Urea SDS-PAGE and digital autoradiography. B. Wild type and ina22 $\Delta$ mitochondria were solubilized in SDS-sample buffer, subjected to SDS-PAGE and Western blotting with the indicated antibodies. C. Wild type and ina22 $\Delta$ mitochondria were subjected to in organello labeling for $20 \mathrm{~min}$ and proteins were co-immunoprecipitated with either control or anti-Atp23 antibodies. Input (1\%) and elution (100\%) were analyzed by Urea SDS-PAGE, digital autoradiography and Western blotting (WB) with anti-Atp23 antibodies.

were decreased in ina22 $\Delta$ and caused the observed processing defect, we analyzed proteins in wild type and ina22 $\Delta$ mutant mitochondria by SDS-PAGE and Western blotting. Surprisingly, the amount of Atp23 in ina22 $\Delta$ mitochondria was similar to the wild type control (Fig. 2.4B) and therefore Atp23 deficiency could not be the reason for Atp6 precursor accumulation. Another possible explanation for the observed Atp6 processing defect could be an impaired interaction between Atp6 and Atp23. Thus, we performed Atp23 immunoprecipitation from wild type and ina22 $\Delta$ mutant mitochondria in which mitochondrial-encoded proteins were labeled with $\left.{ }^{35} \mathrm{~S}\right]$ methionine. Similar amounts of Atp6 were co-immunoprecipitated with Atp23 in ina22 $\Delta$ and wild type mitochondria (Fig. 2.4C), implying that INA22 deletion does not affect Atp6-Atp23 interaction. Therefore, we concluded that neither Atp23 levels, nor Atp23 association with Atp6, is affected upon INA22 deletion and that 
preAtp6 accumulation requires another explanation.

\subsubsection{Atp23 overexpression rescues the Atp6 processing defect in ina22 $\Delta$}

Recent proteomics studies suggest that only 96 molecules of Atp23 are present in the cell - a very low number compared to 20100 molecules of Yme1, another mitochondrial protease (Kulak et al., 2014). Taking into consideration more than a two-fold upregulation of Atp6 expression in ina22 $\Delta$ mutant cells, we wondered whether the detected Atp6 processing defect was due to limited Atp23 amounts in mitochondria. If this were the case, an overexpression of Atp23 should restore Atp6 processing in ina22 $\Delta$. Therefore, a glyceraldehyde 3-phosphate dehydrogenase promoter was inserted upstream of the ATP23 gene in wild type and ina22 $\Delta$ to upregulate protein expression. A steady-state analysis of mitochondrial protein levels in wild type and mutant strains confirmed that this led to a significant increase of Atp23 amounts (Fig. 2.5A). Moreover, this drastic overexpression did not cause protein aggregation in the cytoplasm, as Atp23 was still efficiently imported into mitochondria. Next, we assessed Atp6 processing by pulse-labeling of mitochondrialencoded proteins and analysis by Urea SDS-PAGE and autoradiography. Strikingly, Atp23 overexpression completely restored Atp6 processing in ina22 4 (Fig. 2.5B). However, it only partially rescued the increased Atp6 production rate in ina22 $\Delta$ cells, as still $50 \%$ more protein was synthesized in mutant compared to wild type (Fig. 2.5B, right panel). Therefore, it was concluded that accumulation of preAtp6 in ina22 $\Delta$ was caused by rate limited Atp23 levels and Atp23 overexpression completely restores the Atp6 processing defect, but not the translational upregulation.

\subsubsection{Atp23 overexpression partially rescues $\mathrm{F}_{1} \mathrm{~F}_{\mathrm{o}}$-ATP synthase assem- bly defects in ina22 $\Delta$}

According to Rak et al., 2011, the peripheral stalk of the $\mathrm{F}_{1} \mathrm{~F}_{\mathrm{o}}$-ATP synthase associates with Atp6 and Atp8 in order to form a discrete assembly intermediate. As changes in Atp6 and Atp8 translation, as well as Atp6 processing, were detected in ina mutants, we wondered whether the affected biogenesis of Atp6/Atp8 was contributing to the impaired peripheral stalk assembly in ina mutants. Having seen that an overexpression of Atp23 completely restored Atp6 processing and partially decreased Atp6 upregulation in ina22 $\Delta$, we asked if it would also have an effect on $\mathrm{F}_{1} \mathrm{~F}_{\mathrm{o}}$-ATP synthase assembly. Therefore, we assessed $\mathrm{F}_{1} \mathrm{~F}_{\mathrm{o}}$-ATP synthase levels in wild type, ina22 $\Delta$ and Atp23个ina22 $\Delta$ mutant mitochondria by BN-PAGE and subsequent Western blotting or in-gel activity staining. Western blot analysis with anti-Atp4 antibodies clearly showed that Atp23 overexpression restored $\mathrm{F}_{1} \mathrm{~F}_{\mathrm{o}}$-ATP synthase levels in ina22 $\Delta$ (Fig. 2.6A, lanes 1-4). Moreover, in-gel activity staining 
A

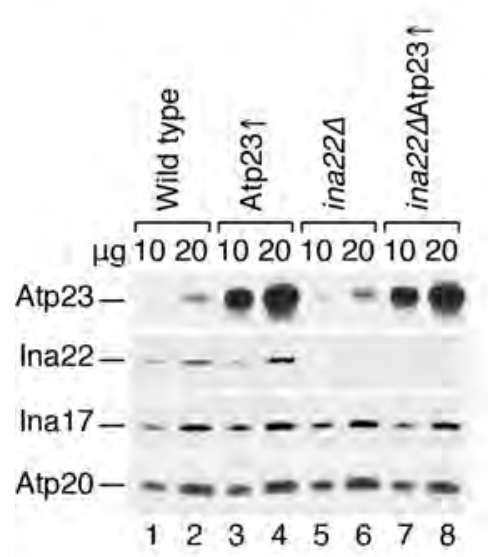

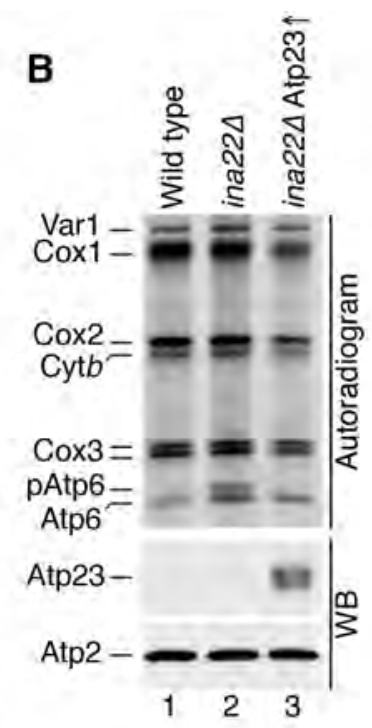

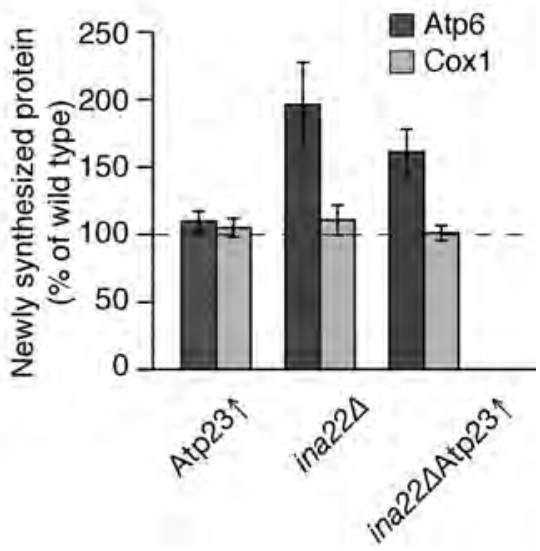

Figure 2.5 Atp23 overexpression rescues the Atp6 processing defect in ina22 $\Delta$. A. Steady-state protein levels in wild type and mutant mitochondria were analyzed by SDS-PAGE and Western blotting with the indicated antibodies. B. Mitochondrialencoded proteins were radiolabeled in vivo for $10 \mathrm{~min}$ and analyzed by Urea SDSPAGE and digital autoradiography, or by Western blotting (WB) with the indicated antibodies. Quantification was performed as in Fig. 2.1A. ( $\mathrm{n}=3, \pm \mathrm{SEM})$.

confirmed reduced amounts of unassembled $\mathrm{F}_{1}$ in Atp23个ina22 $\Delta$ (Fig. 2.6A, lanes 58). To check whether this improvement was due to recovered assembly of $\mathrm{F}_{1} \mathrm{~F}_{\mathrm{o}}$-ATP synthase, the Atp4 precursor protein was synthesized in vitro in the presence of $\left.{ }^{35} \mathrm{~S}\right]$ methionine, imported into wild type and mutant mitochondria and its incorporation into complex $\mathrm{V}$ was analyzed by BN-PAGE and autoradiography. As can be seen from the result, Atp4 assembly is severely compromised in ina22 $\Delta$ mitochondria, while Atp23 overexpression leads to its partial recovery, as more complex V could be detected in Atp23个ina22 (Fig. 2.6B).

Another prominent feature of ina mutants is decreased $\mathrm{F}_{1} \mathrm{~F}_{\mathrm{o}}$-ATP synthase oligomycin sensitivity due to increased levels of free $F_{1}$. To address whether improved $\mathrm{F}_{1} \mathrm{~F}_{\mathrm{o}}$-ATP synthase assembly and decreased amounts of unassembled $\mathrm{F}_{1}$ in Atp23个ina22 $\Delta$ correspond to restored oligomycin sensitivity, we performed ATPase activity measurements in wild type and mutant strains. Strikingly, oligomycin insensitivity of ina22 $\Delta$ was reduced almost two-fold upon Atp23 overexpression (Fig. 2.6C). Taken together, these data imply that Atp23 overexpression partially rescues $\mathrm{F}_{1} \mathrm{~F}_{\mathrm{o}}$-ATP synthase assembly in ina22 $\Delta$ mutant.

Atp23 has a dual function in Atp6 processing and assembly (Osman et al., 2007). It was shown that in yeast expressing catalytically-inactive Atp23 the mature $\mathrm{F}_{1} \mathrm{~F}_{\mathrm{o}}$-ATP synthase is still assembled, implying that the proteolytic activity of 
A

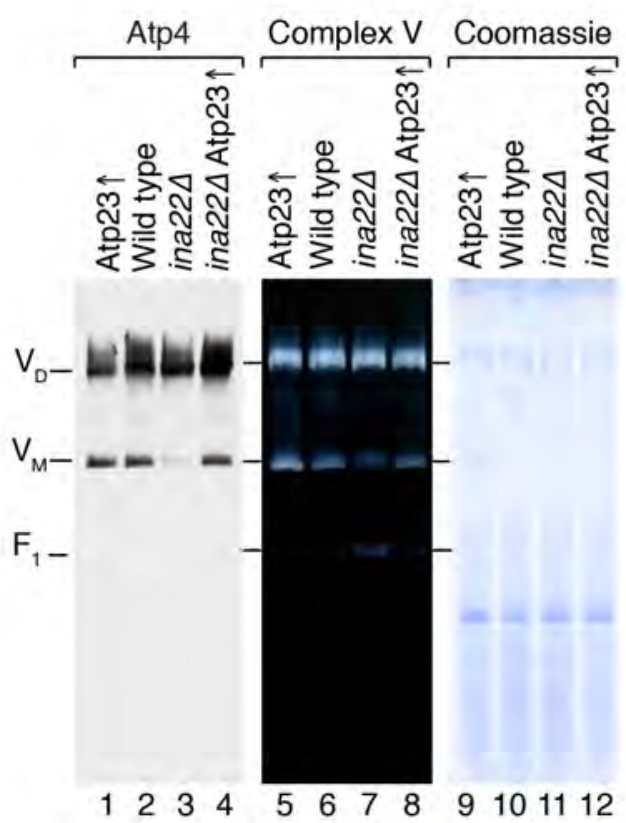

B

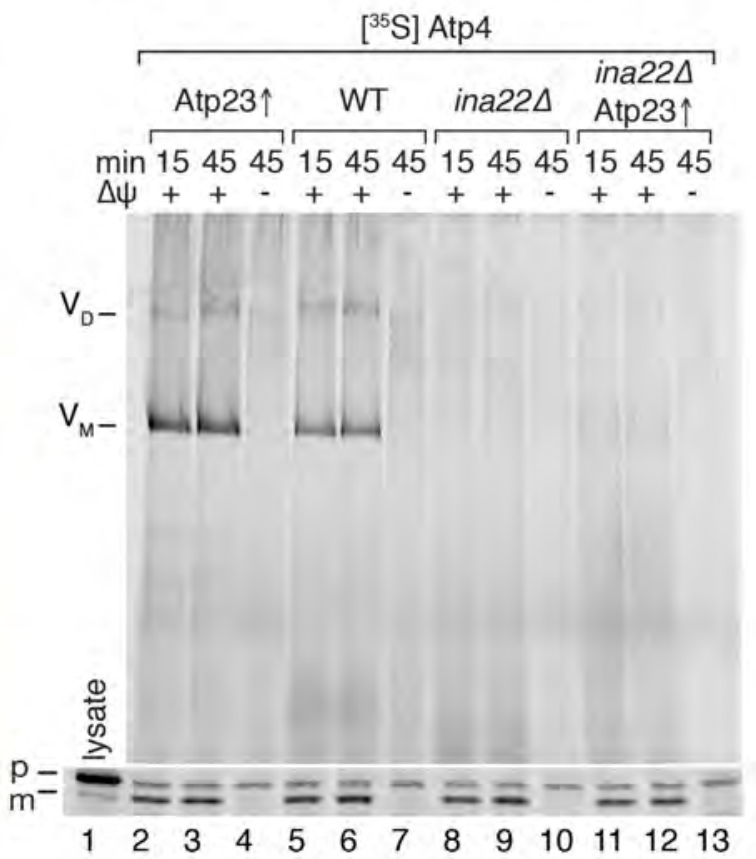

C

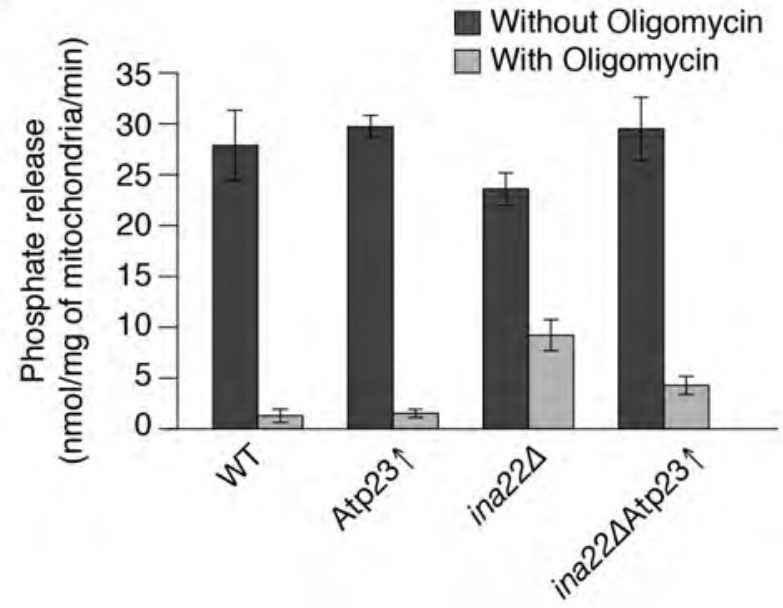

Figure 2.6 Atp23 overexpression partially rescues $F_{1} F_{o}$-ATP synthase assembly in ina22 $\Delta$. A. Mitochondria were analyzed by Blue Native (BN)-PAGE, followed by Western blotting with anti-Atp4 antibodies (lanes 1-4), in-gel ATPase activity staining (Complex V, lanes 5-8), or Coomassie staining (lanes 9-12). B. Radiolabeled Atp4 precursor protein was imported into wild type and mutant mitochondria with or without membrane potential. Samples were analyzed by BN-PAGE (top panel) or SDS-PAGE (bottom panel) and digital autoradiography. p, precursor; m, mature; WT, wild type C. In organello ATPase activity measurement was performed as described in Materials and Methods in wild-type and mutant mitochondria, with or without addition of $50 \mu \mathrm{M}$ oligomycin. Activity is depicted as nmoles of released phosphate per min per $\mathrm{mg}$ of mitochondrial proteins $(\mathrm{n}=5, \pm$ SEM $)$.

Atp23 is not essential (Osman et al., 2007). However, Atp23 function in assembly of complex $\mathrm{V}$ is vital. We have observed both Atp6 processing rescue, as well as an im- 
A

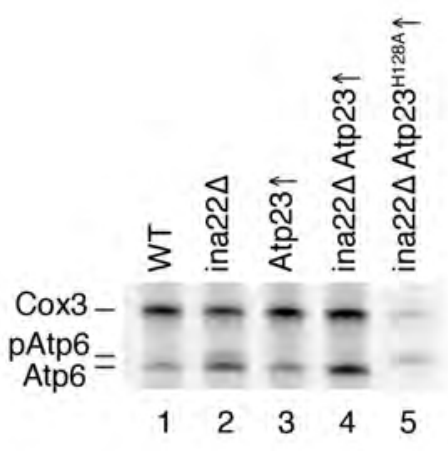

C

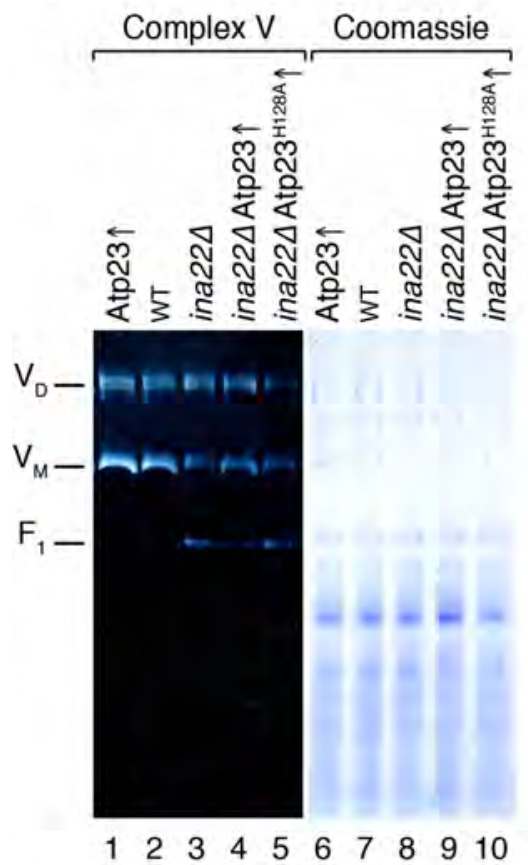

B
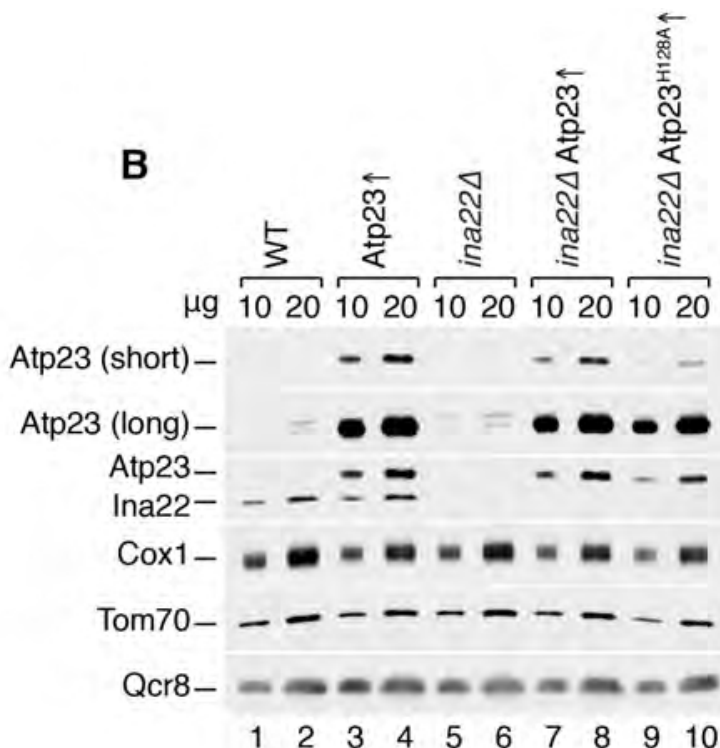

D

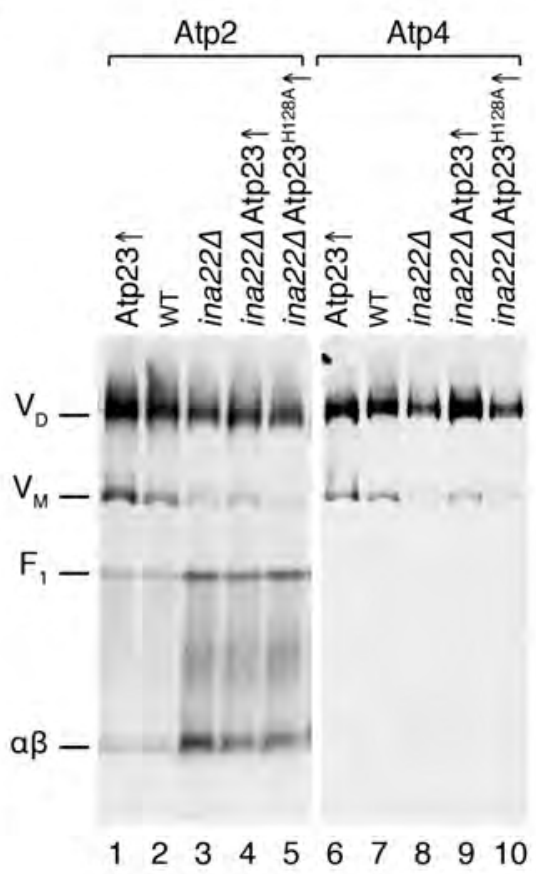

Figure 2.7 Atp23 ${ }^{\mathrm{H128A}}$ overexpression does not restore the $\mathrm{F}_{1} \mathrm{~F}_{\mathrm{o}}$-ATP synthase assembly defect in ina22 $\Delta$. A. Mitochondrial-encoded proteins in wild type and mutant mitochondria were radiolabeled for 10 minutes and analyzed by Urea SDSPAGE and autoradiography. B. Steady-state protein levels in wild type and mutant mitochondria were analyzed by SDS-PAGE and Western blotting with the indicated antibodies. C. Wild type and mutant mitochondria were solubilized, analysed by BNPAGE and either in-gel activity staining (Complex V) or Coomassie staining. D. Wild type and mutant mitochondria were analyzed by BN-PAGE and Western blotting with indicated antibodies.

provement of $\mathrm{F}_{1} \mathrm{~F}_{\mathrm{o}}$-ATP synthase assembly in ina22 $\Delta$ upon Atp23 overexpression. To assess whether overexpression of catalytically-inactive Atp23 would still result 
in ina22 $\Delta$ phenotype rescue, we generated a strain overexpressing Atp23 with a point mutation in its catalytic site $\left(\operatorname{Atp} 23^{\mathrm{H} 128 \mathrm{~A} \uparrow}\right)$ in the ina22 $\Delta$ background. In vivo pulse-labeling analysis of mitochondrial-encoded proteins shows that preAtp6 is synthesized in this strain, however, it is not processed to mature Atp6 (Fig. 2.7A, lane 5). Surprisingly, a Western blot analysis of mitochondrial steady-state protein levels showed that Atp23 $3^{\mathrm{H} 128 \mathrm{~A}}$ is rather unstable, as its amounts were reduced compared to the amounts of overexpressed wild type Atp23 (Fig. 2.7B). Further BNPAGE and in-gel activity staining analysis of wild type and mutant mitochondria revealed that overexpression of Atp2 $3^{\mathrm{H} 128 \mathrm{~A}}$ reduced neither the levels of unassembled $\mathrm{F}_{1}$ (Fig. 2.7C), nor restored the steady-state levels of mature $\mathrm{F}_{1} \mathrm{~F}_{\mathrm{o}}$-ATP synthase (Fig. 2.7D) in ina22 $\Delta$ mitochondria. Therefore we conclude that overexpression of Atp23 ${ }^{\mathrm{H} 128 \mathrm{~A}}$ does not rescue the ina22 $\Delta$ phenotype. However, we could not distinguish whether it was due to the impaired Atp6 processing or increased Atp23 ${ }^{\mathrm{H} 128 \mathrm{~A}}$ instability.

\subsection{The C-terminus of Ina22 is highly unstable}

In order to avoid using tagged variants of Ina17 and Ina22, we generated antibodies against the IMS domain of Ina22 and the matrix domain of Ina17. Interestingly, during initial immunoprecipitations two distinct Ina22 fragments, which represent either products of protein processing or degradation, were co-isolated along with the full-length Ina22 from wild type mitochondria (Fig. 2.8A). Notably, these fragments were also isolated from ina17 $\Delta$ mitochondria, implying that the observed processing is not Ina17-dependent (Fig. 2.8A, lane 7). Moreover, the fragments were co-immunoprecipitated with Ina17, meaning that they still contain the coiled-coil region required for Ina17-Ina22 interaction (Lytovchenko et al., 2014). Importantly, the same fragments were not reproducibly detected in intact mitochondria and were usually highly enriched after affinity purification. The latter implies that Ina22 is much more unstable during isolation conditions, which might be due to increased protein instability or protease processing. In order to identify the protease(s) responsible for Ina22 processing, we screened yeast mutants, in which known IMS proteases were deleted, for Ina22 processing defects. Unfortunately, no potential candidates were identified (data not shown; performed in collaboration with Dr. Nora Vögtle).

To distinguish between $\mathrm{C}$ - and N-terminal processing, Ina22 was immunoprecipited from WT, ina22 $\Delta$ and Ina22 ${ }^{\mathrm{HA}}$ mitochondria containing a C-terminal HA tag. Only full-length Ina22 could be detected with anti-HA antibodies in both the input and elution fractions (Fig 2.8B, upper panel), whereas decoration with antiIna22 antibodies confirmed the presence of two additional fragments in the elution (Fig. 2.8B, lower panel). Thus, it was clarified that Ina22 undergoes C-terminal pro- 
A

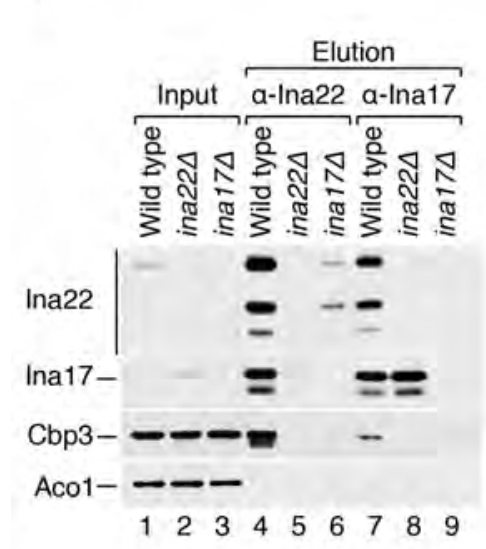

D

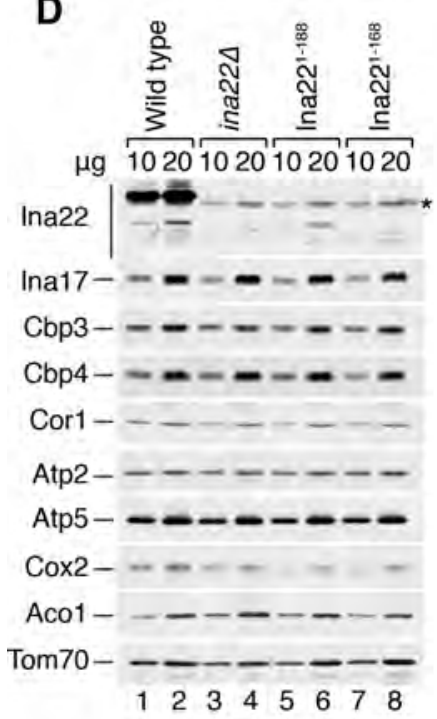

B

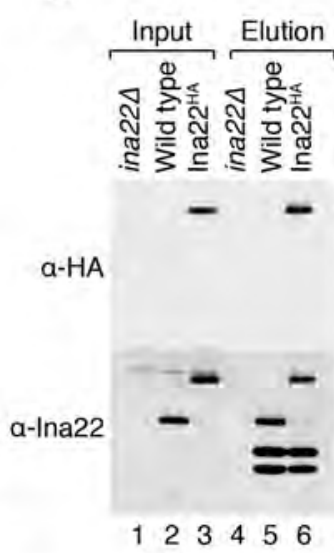

C

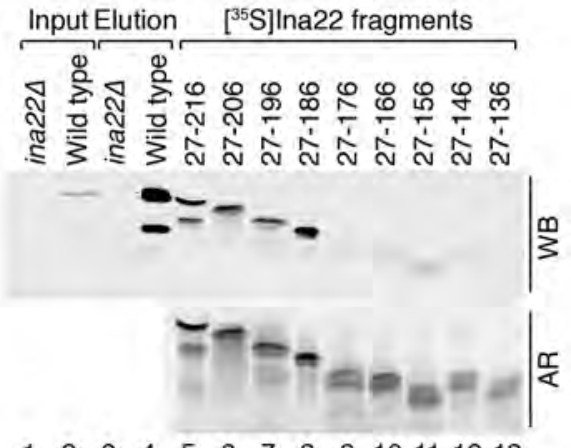

$\begin{array}{llllllllllllll}1 & 2 & 3 & 4 & 5 & 6 & 7 & 8 & 9 & 10 & 11 & 12 & 13\end{array}$

E

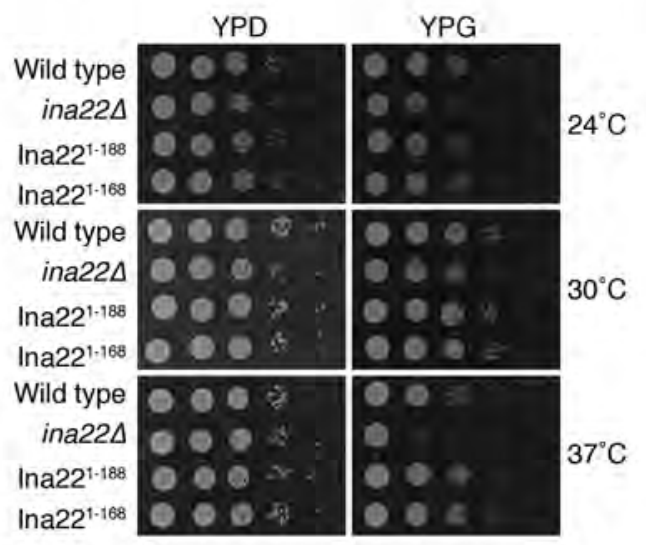

Figure 2.8 Ina22 undergoes C-terminal processing. A. Wild type, ina22 $\Delta$ and ina17 $\Delta$ mitochondria were solubilized and protein complexes were co-immunoprecipitated with anti-Ina22 and anti-Ina17 antibodies. Input and elution fractions were analyzed by SDS-PAGE and Western blotting with the indicated antibodies. Input = $1 \%$ of elution. B. ina22 $\Delta$, wild type and Ina $22^{\mathrm{HA}}$ mitochondria were subjected to co-immunoprecipitation with anti-Ina22 antibodies. Input and elution fractions were analyzed by SDS-PAGE and Western blotting with either anti-HA or anti-Ina22 antibodies. Input $=1 \%$ of elution. C. Ina 22 was immunoprecipitated from wild type and ina22 $\Delta$ mitochondria with anti-Ina22 antibodies and the molecular weight of its fragments was assessed by comparing their electrophoretic mobility (lanes 1-4) to the mobility of in vitro generated radiolabeled Ina22 fragments (lanes 5-13). Samples were analyzed by SDS-PAGE, digital autoradiography (AR) and Western blotting (WB). D. Mitochondrial proteins in wild type and mutant mitochondria were analyzed by SDS-PAGE and Western blotting with the indicated antibodies. The asterisk $(*)$ indicates an unspecific band. E. A serial dilution of yeast cells was spotted on YPD or YPG plates. Plates were grown at the indicated temperatures for 2-5 days.

cessing. In order to define the length of the fragments, we generated in vitro a set of C-terminally truncated radiolabeled Ina22 fragments (devoid of a predicted import signal) and compared their electrophoretic mobility to the mobility of immunopre- 
cipitated Ina22 fragments (Fig. 2.8C). We identified that the processing takes place approximately at amino acids 168 and 188 of Ina22. To further investigate the potential function of the processing, we generated yeast mutants expressing truncated Ina22, either 168 or 188 amino acids in length, and analyzed the steady-state levels of selected mitochondrial proteins by SDS-PAGE and Western blotting. No alteration of protein levels was observed in the mutant strains (Fig. 2.8D) and their growth on non-fermentable media was not compromised (Fig. 2.8E). Therefore, we conclude that the extreme C-terminus of Ina22 does not contribute to its function and that the biological importance of the C-terminal processing/degradation remains to be identified.

\subsection{INAC interacts with the Atp6/Atp8 biogenesis machin- ery}

\subsubsection{INAC associates with Atp23}

After we identified that the early biogenesis of Atp6 and Atp8 was altered in ina mutants, we asked whether Ina17 and Ina22 are directly involved in this process. To address this, we assessed if Ina proteins associate with known components of the Atp6/Atp8 assembly machinery, namely Atp23 and Atp10. First, we performed immunoprecipitation of Ina22 and Ina17 with antibodies targeting the endogenous proteins and analyzed elution fractions for the presence of Atp23. Clearly, Atp23 co-isolated with INAC, confirming their physical association (Fig. 2.9A).

To assess whether this interaction depends on mitochondrial-encoded proteins, we generated a yeast stain devoid of Atp6 by deleting ATP22, gene encoding Atp6specific translational activator (Zeng et al., 2007a). Mitochondria from atp22 $\Delta$ and control wild type yeast strains were isolated and subjected to immunoprecipitation with anti-Ina22 and anti-Ina17 antibodies. ATP22 deletion and the resulting absence of Atp6 significantly reduced the INAC-Atp23 interaction, but did not abolish it (Fig. 2.9B). Nevertheless, the obtained results were hard to interpret as steadystate levels of mitochondrial proteins in atp22 $\Delta$ were significantly altered, most probably due to the genetic instability of its mitochondrial genome. Therefore, to confirm that the INAC-Atp23 association depends on mitochondrial-encoded proteins, we asked whether upregulation of mitochondrial translation, and the subsequent accumulation of early assembly intermediates of complex $\mathrm{V}$, would increase INAC interaction with Atp23. Chloramphenicol treatment prior to mitochondrial isolation was shown to result in accumulation of nuclear gene products within the organelle. The subsequent removal of chloramphenicol and release of the translational block enhances mitochondrial gene expression (Tzagoloff, 1971; Osman et al., 2007; Rak et al., 2011). Therefore, wild type yeast cells were grown with or without a 2-h 
A

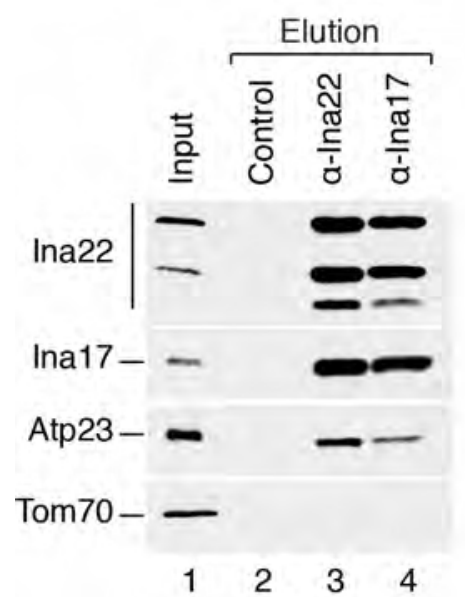

B
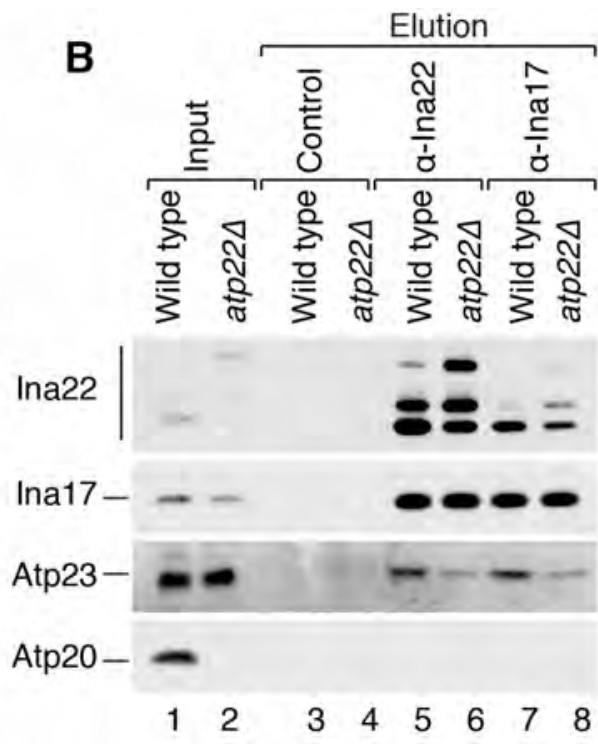

Figure 2.9 INAC interacts with Atp23. A. Proteins from wild type mitochondria were coimmunoprecipitated with control, anti-Ina22 or anti-Ina17 antibodies. Input and elution fractions were analyzed by SDS-PAGE and Western blotting with the indicated antibodies. Input $=1 \%$ of elution. B. Proteins from wild type and atp22 $\Delta$ mitochondria were co-immunoprecipitated with control, anti-Ina22 and anti-Ina17 antibodies and analyzed as in A. Input $=1 \%$ of elution.

preincubation with chloramphenicol. Mitochondria from these cells were isolated and the translation of mitochondrial-encoded proteins was analyzed via in organello labeling, SDS-PAGE and autoradiography. Mitochondrial translation was clearly enhanced in chloramphenicol-pretreated samples compared to the non-treated control (Fig. 2.10A, lanes 1-3 versus 4-6). Importantly, when chloramphenicol was not washed away during mitochondrial preparation, mitochondrial translation was completely inhibited, confirming the specificity of treatment (Fig. 2.10A, lanes 79 ). Next, the control and chloramphenicol-treated mitochondria were subjected to immunoprecipitation with anti-Ina22 and anti-Ina17 antibodies. As can be seen, more Atp23 co-isolated with INAC upon upregulation of mitochondrial translation (Fig. 2.10B). This implies that their interaction is mediated by newly translated, mitochondrial-encoded, $\mathrm{F}_{1} \mathrm{~F}_{\mathrm{o}}$-ATP synthase subunits.

\subsubsection{INAC interacts with Atp10}

Atp10 was previously shown to associate with newly translated Atp6, to stabilize it and to facilitate its assembly (Tzagoloff et al., 2004; Rak et al., 2011). For verification of a possible Atp10-INAC interaction, we genomically fused protein A from $S$. aureus to the C-terminus of Atp10. To address whether the protein remained functional, we analyzed Atp $10^{\text {ProtA }}$ growth on a non-fermentable carbon source and did not observe any differences compared to wild type (Fig. 2.11A). Moreover, a BN-PAGE analysis of respiratory chain complexes in wild type and Atp10 ${ }^{\text {ProtA }}$ mito- 


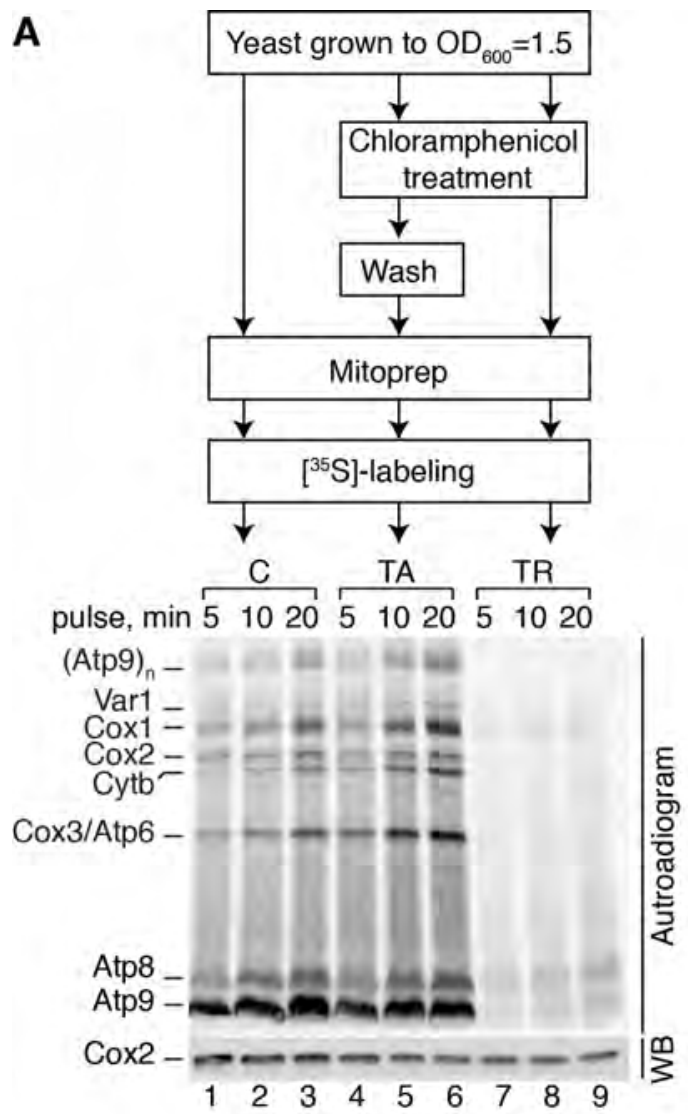

\section{B}

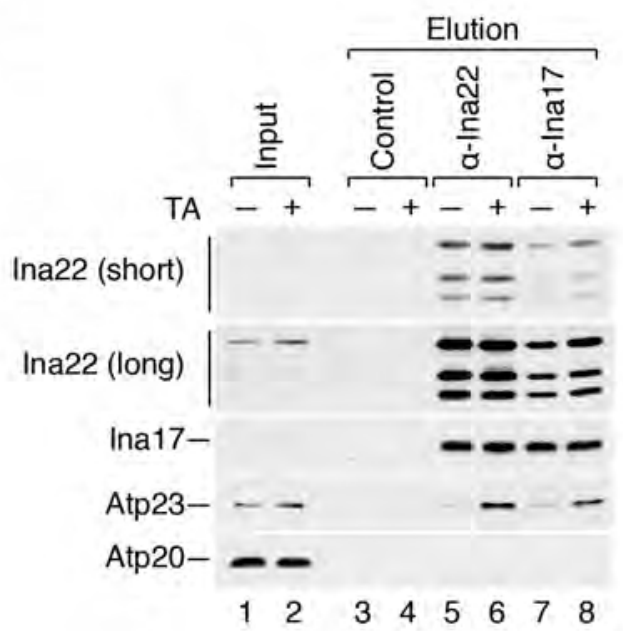

Figure 2.10 Upregulation of mitochondrial translation increase INAC-Atp23 association. A. Yeast cells were treated for 3 hours with $2 \mathrm{mg} / \mathrm{ml}$ chloramphenicol or left untreated $(\mathrm{C}$, control). Then, chloramphenicol was washed away and cells were split in two parts for mitochondrial isolation. Mitochondria from the first part were isolated according to the standard protocol (TA, translationally-activated). For the mitochondria isolation from the second part, all buffers were supplemented with 2 $\mathrm{mg} / \mathrm{ml}$ chloramphenicol (TR, translationally-repressed). Isolated mitochondria were subjected to in organello radiolabeling and samples were analyzed by SDS-PAGE and autoradiography. B. Mitochondrial-encoded products in control and translationallyactivated (TA) mitochondria were labeled in organello. Mitochondria were solubilized and subjected to immunoprecpitation with control, anti-Ina22 and anti-Ina17 antibodies. Samples were analyzed by SDS-PAGE and autoradiography. Input $=1 \%$ of elution. "short" and "long" refers to different exposure time.

chondria confirmed their comparable steady-state amounts (Fig. 2.11B). Therefore, we concluded that tagged Atp10 is functional. To assess the INAC-Atp10 interaction and whether it is mediated by mitochondrial translation products, we isolated Atp10 ${ }^{\text {ProtA }}$-associated protein complexes from control and chloramphenicol-treated mitochondria by IgG-affinity chromatography and analyzed them by SDS-PAGE and Western blotting. As a result, Ina17 and Ina22 co-isolated with Atp10 ${ }^{\text {ProtA }}$, confirming INAC association with the Atp6/Atp8 biogenesis machinery (Fig. 2.12A). Importantly, a significant increase in their interaction was observed upon mitochondrial translational upregulation, implying again that the INAC interaction with Atp10, as well as with Atp23, involves mitochondrial gene products. Moreover, newly im- 
A

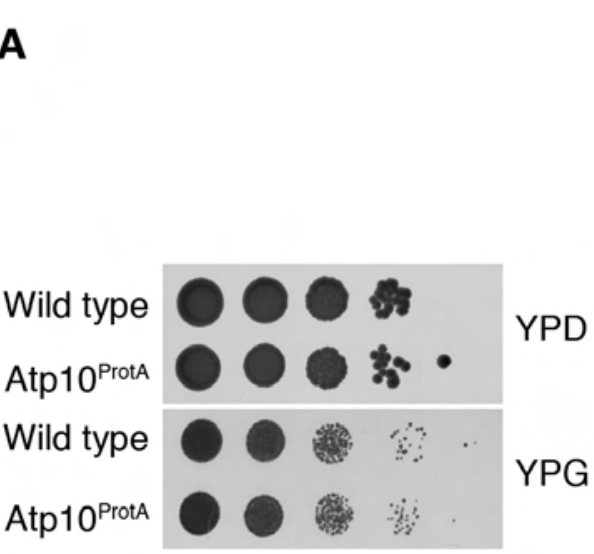

B
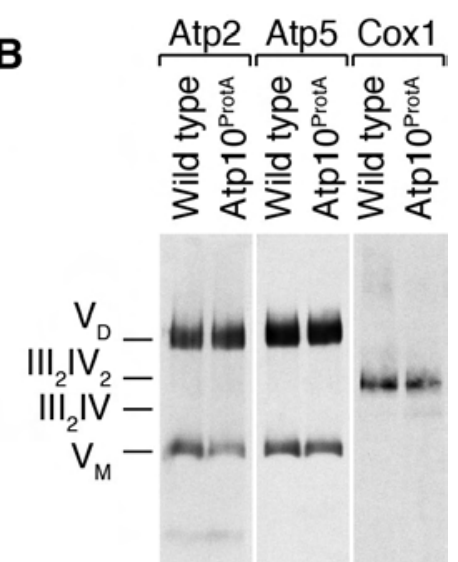

$123 \quad 3 \quad 4 \quad 56$

Figure 2.11 C-terminal tagging does not affect Atp10 function. A. A serial dilution of yeast cells was spotted on YPD or YPG plates. Plates were grown at $37^{\circ} \mathrm{C}$ for 5 days. B. Mitochondria isolated from wild type and Atp $10^{\text {ProtA }}$ strains were analyzed by BN-PAGE and Western blotting with the indicated antibodies.

ported radiolabeled Atp10 was co-isolated with Ina22 ${ }^{\mathrm{FLAG}}$, confirming again their interaction (Fig. 2.12B).

Surprisingly, Atp23 was co-isolated with Atp10 ${ }^{\text {ProtA }}$, in contrast to previous findings (Rak et al., 2011) (Fig. 2.12A). Our result proves a previously proposed Atp23 function in the assembly of Atp6 (Osman et al., 2007), as it remains associated with the Atp10/Atp6 complex during later assembly steps. Nonetheless, the Atp10Atp23 interaction was not changed upon mitochondrial translational upregulation, implying that the Atp10-Atp23 association is more stable and that these two proteins might interact with each other even in absence of Atp6.

To address whether INAC interacts with Atp10 and Atp23 in a distinct protein complex, we purified Atp10 ${ }^{\text {ProtA }}$ complexes from chloramphenicol-pretreated mitochondria via IgG affinity chromatography. Bound protein complexes were eluted natively via TEV-protease cleavage and analyzed by BN-PAGE and Western blotting. Strikingly, an Atp23, Ina22 and Ina17-containing complex of 400 kDa was detected, in which all three proteins co-migrated (Fig. 2.12C). However, protein complexes in which only Atp23 was present, were much more abundant, implying again that the Atp10-Atp23 interaction is much more robust, whereas the Atp10-INAC interaction is transient in nature.

Thus, we conclude that INAC interacts with Atp23 and Atp10. Moreover, as we did not know the exact nature of the isolated complex, we speculated that this is one of the $\mathrm{F}_{1} \mathrm{~F}_{\mathrm{o}}$-ATP synthase assembly intermediates, most probably the Atp6/Atp8 assembly module. 
A

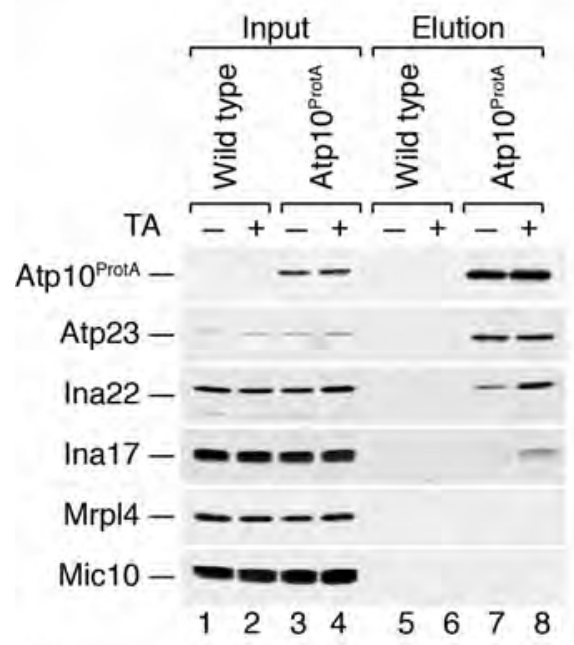

B

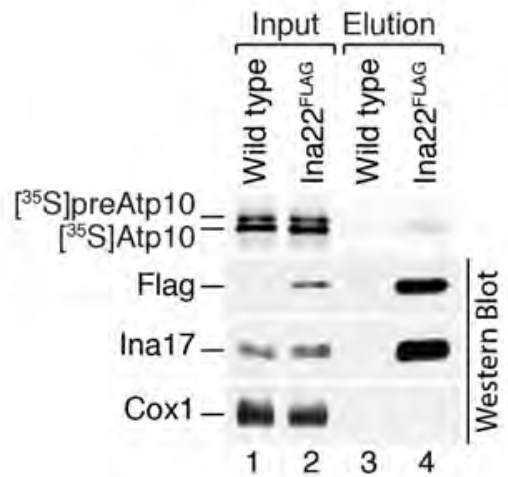

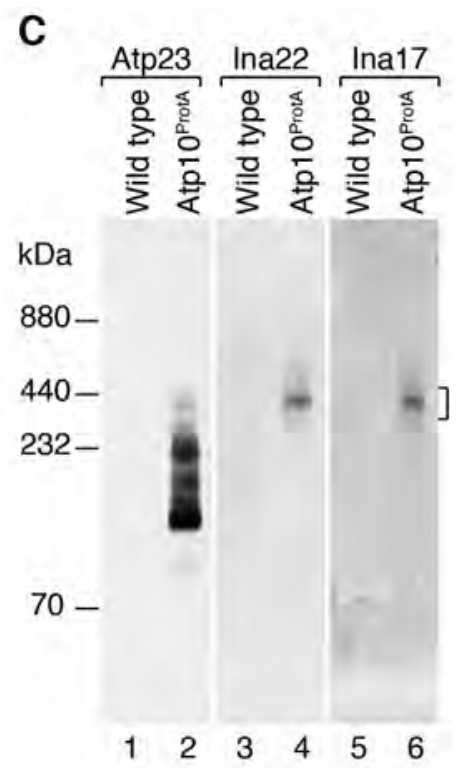

Figure 2.12 INAC associates with Atp10. A. Control and chloramphenicol-treated wild type and Atp $10^{\operatorname{ProtA}}$ mitochondria were subjected to IgG affinity chromatography and analyzed by SDS-PAGE and Western blotting with the indicated antibodies. Input $=1 \%$ of elution. TA, translational activation. B. Atp10 precursor (preAtp10) was synthesized in vitro in the presence of $\left[{ }^{35} \mathrm{~S}\right]$ Met and imported into wild type or Ina $22^{\text {FLAG }}$ mitochondria. Mitochondria were then subjected to anti-FLAG affinity chromatography. Input and elution fractions were analyzed by SDS-PAGE and digital autoradiography (upper panel) or Western blotting with the indicated antibodies. Input $=1 \%$ of elution. C. Protein complexes from Atp $10^{\text {ProtA }}$ and wild type mitochondria were purified via IgG affinity chromatography, eluted natively via TEV-cleavage and analyzed by BN-PAGE and Western blotting with the indicated antibodies. Chloramphenicol-treated mitochondria were used. 


\subsection{Stalling of $\mathrm{F}_{1} \mathrm{~F}_{\mathrm{o}}$-ATP synthase assembly in ina mutants}

\subsubsection{Atp10 accumulates with Atp23 upon INA22 deletion}

According to Rak et al., 2011, the peripheral stalk, Atp6, Atp8 and Atp10 together form a preassembled module that does not contain Atp23. However, our data clearly show that Atp10 stably associates with Atp23 as part of multiple protein complexes, some of which contain Ina proteins, as well as some that do not (Fig. $2.12 \mathrm{C}$ ). According to our initial assumption, INAC associates with the Atp6/Atp8 module in order to facilitate peripheral stalk assembly.

A

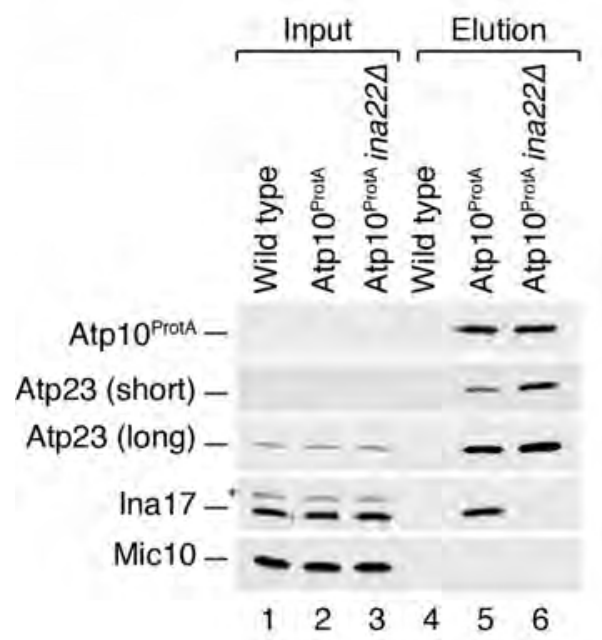

B

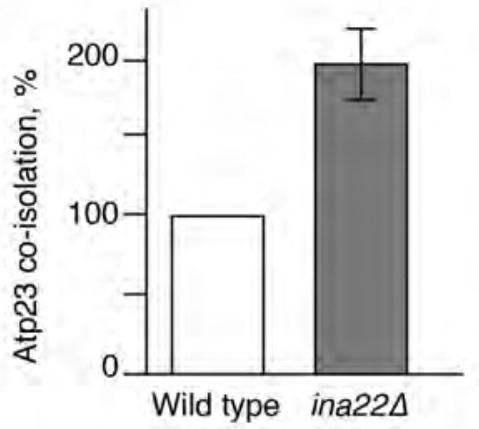

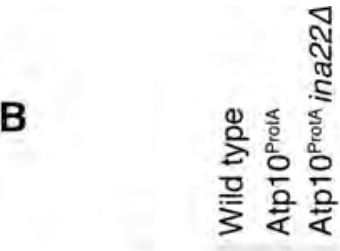

$\mathrm{kDa}$

$880-$

$440-$

$232-$

123

Figure 2.13 Atp23 accumulates with Atp10 upon INA22 deletion. A. Atp10 ProtA containing protein complexes were purified via IgG affinity chromatography from Atp $10^{\text {ProtA }}$, Atp10 ${ }^{\text {ProtA }}$ ina22 $\Delta$ and wild type mitochondria. Input and elution fractions were analyzed by SDS-PAGE and Western blotting with the indicated antibodies (left panel). The amount of Atp23 that was isolated from Atp10 ${ }^{\text {ProtA }}$ mitochondria was set as $100 \%$ and Atp23 co-isolated from Atp10 ${ }^{\text {ProtA }}$ ina22 $\Delta$ mitochondria was quantified (right panel). Signals were normalized to the amount of isolated Atp $10^{\text {ProtA }}$. Input $=1 \%$ of elution. $\mathrm{n}=3, \pm$ SEM. B. The experiment was performed as in panel A, protein complexes were eluted via TEV protease cleavage and elution fractions were analyzed by BN-PAGE and Western blotting with anti-Atp23 antibodies.

Thus, we wondered whether deletion of the INA complex would lead to a stall of assembly and the accumulation of Atp10 and Atp23-containing assembly intermediates. Therefore, Atp $10^{\text {ProtA }}$ complexes were isolated from wild type and ina22 $\Delta$ mitochondria and analyzed by SDS-PAGE and Western blotting. As expected, Atp23 accumulated with Atp10 in ina22 $\Delta$, strongly suggesting that they are part of the same assembly intermediate prior to interaction with INAC (Fig. 2.13A). More- 
over, a BN-PAGE analysis of the elution fractions, followed by Western blotting with anti-Atp23 antibodies clearly showed a prominent accumulation of the Atp10and Atp23-containing protein complex at around $230 \mathrm{kDa}$ in ina22 $\Delta$ mitochondria (Fig. 2.13B). Thus, in contrast to previously published results, Atp10 and Atp23 interact with each other. Moreover, these two proteins are components of the same $\mathrm{F}_{1} \mathrm{~F}_{\mathrm{o}}$-ATP synthase assembly intermediate that accumulates upon INA22 deletion.

\subsubsection{Atp23 association with the IMM is increased in ina22}

Atp23 is a protease of the intermembrane space that peripherally associates with the inner mitochondrial membrane (Osman et al., 2007). Taking the known Atp23 function in Atp6 processing and assembly into consideration, we wondered if Atp6 helps to anchor Atp23 to the membrane. Keeping in mind that Atp6 translation in ina mutants is increased more than two-fold, it was interesting to assess whether this leads to increased Atp23 association with the IMM. Therefore, extraction of mitochondrial proteins using alkaline conditions was performed in wild type and ina22 $\Delta$ mitochondria. Upon sonication, Atp23 remained exclusively in the membrane pellet fraction, similar to two other peripheral membrane proteins, Tim44 and Cbp3 (Fig. 2.14A, lanes 1-3, 7-9). However, upon high pH treatment, Atp23 was extracted from the membrane and therefore detected in the soluble fraction (Fig. 2.14A). Remarkably, in ina22 $\Delta$ mitochondria, more Atp23 remained membrane associated compared to wild type (Fig. 2.14A, lanes 5-6 versus lanes 11-12), whereas association of Cbp3 and Tim44 was unchanged. To verify whether increased Atp23 membrane association was due to the increased Atp6 abundance, the same experiment was repeated in non-treated and chloramphenicol-treated mitochondria that have elevated expression of all mitochondrial-encoded genes (Fig. 2.10A). Surprisingly, a similar result was obtained, namely more Atp23 remained membrane-associated upon increased mitochondrial translation (Fig. 2.14B). To find out whether this effect is Atp6-specific, carbonate extraction was performed from wild type and atp22 4 mitochondria, devoid of Atp6. As expected, ATP22 deletion, and subsequent Atp6 absence, decreased Atp23 association with the IMM. Therefore, we concluded that Atp23 is anchored to the IMM via Atp6 and it accumulates on the membrane upon INA22 deletion due to increased Atp6 translation. Taking into consideration previous results, we assumed that Atp23 accumulates together with Atp10 in an Atp6/Atp8-containing assembly intermediate.

\subsubsection{The Atp6/Atp8 module accumulates upon INA22 deletion}

All previously obtained results point to the fact that Atp10 and Atp23 associate together in an assembly intermediate that will later assemble with the peripheral stalk. Moreover, our data indicate that Atp10 accumulates with Atp23 in ina mu- 
A

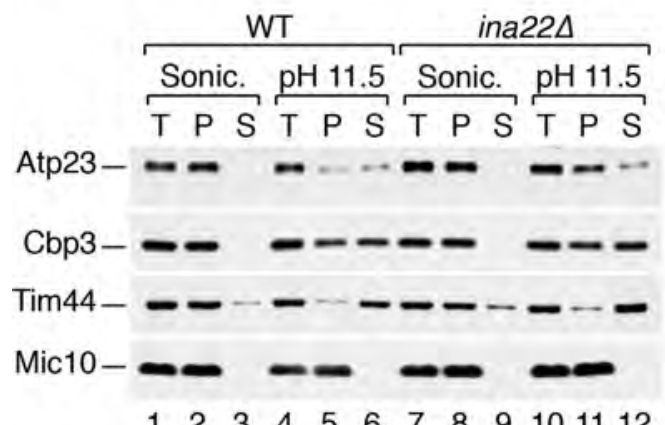

B

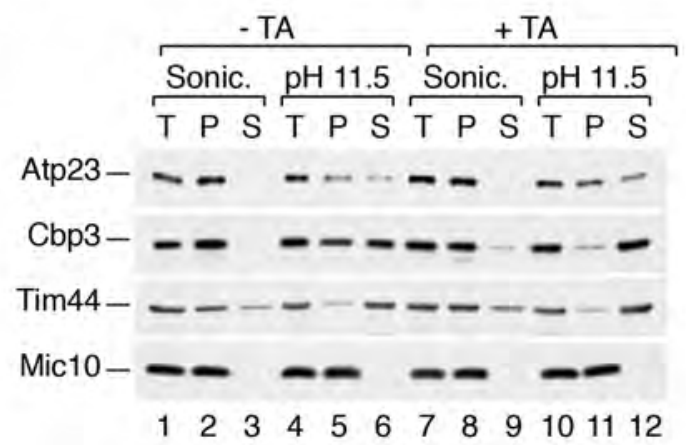

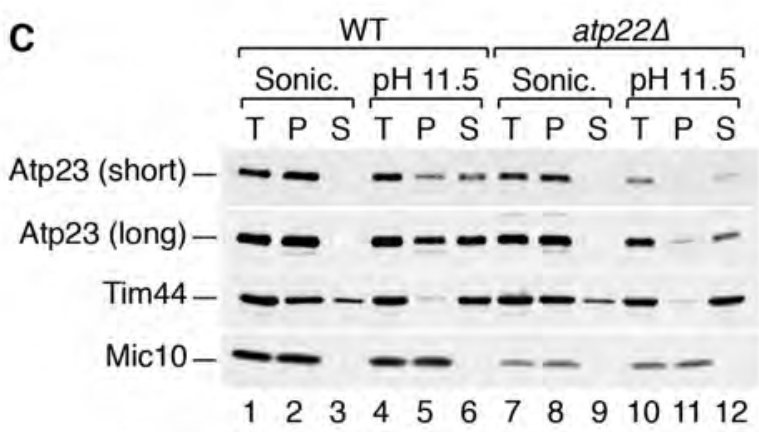

Figure 2.14 Atp23 membrane association increases in ina224. A. Wild type and ina22 mitochondria were either sonicated or treated with carbonate buffer with $\mathrm{pH}$ 11.5. Samples were centrifuged to separate pellet from soluble fraction. Proteins were precipitated with $12 \%$ TCA and analyzed by SDS-PAGE and Western blotting with the indicated antibodies. B. The experiment was performed and analyzed as in panel A with control (-TA) and chloramphenicol-treated mitochondria (+TA). TA, translational activation. C. The experiment was performed and analyzed as in panel A with wild type and atp22 $\Delta$ mitochondria. T, total; $\mathrm{P}$, pellet; S, supernatant.

tants, implying that the assembly process is partially stalled at this point. If so, newly-translated mitochondrial-encoded Atp6 and Atp8 should accumulate in the same complex with Atp10 and Atp23 in ina22 $\Delta$.

To address whether this was the case, we radiolabeled mitochondrial-encoded proteins in wild type, Atp10 ${ }^{\text {ProtA }}$ and Atp10 ${ }^{\text {ProtA }}$ ina22 $\Delta$ chloramphenicol-treated mitochondria and performed an isolation of Atp $10^{\text {ProtA }}$-containing protein complexes. As a result, double the amount of Atp6 and Atp8 were co-isolated with Atp10 from ina22 $\Delta$ mitochondria compared to wild type (Fig. 2.15A), similar to the increase of the Atp10-Atp23 interaction (Fig. 2.13A). This confirmed our initial assumption about the accumulation of an Atp6/Atp8-containing assembly intermediate in ina mutants. Moreover, to visualize accumulated Atp6 and Atp8-containing protein complexes, we analyzed part of the Atp10 ${ }^{\text {ProtA }}$ elution by BN-PAGE and digital autoradiography. Interestingly, one prominent assembly intermediate of $400 \mathrm{kDa}$ was co-isolated from wild type and ina22 $\Delta$ mitochondria, whereas several additional smaller-size assembly intermediates accumulated in ina22 $\Delta$ (Fig. 2.15B). Further 
A
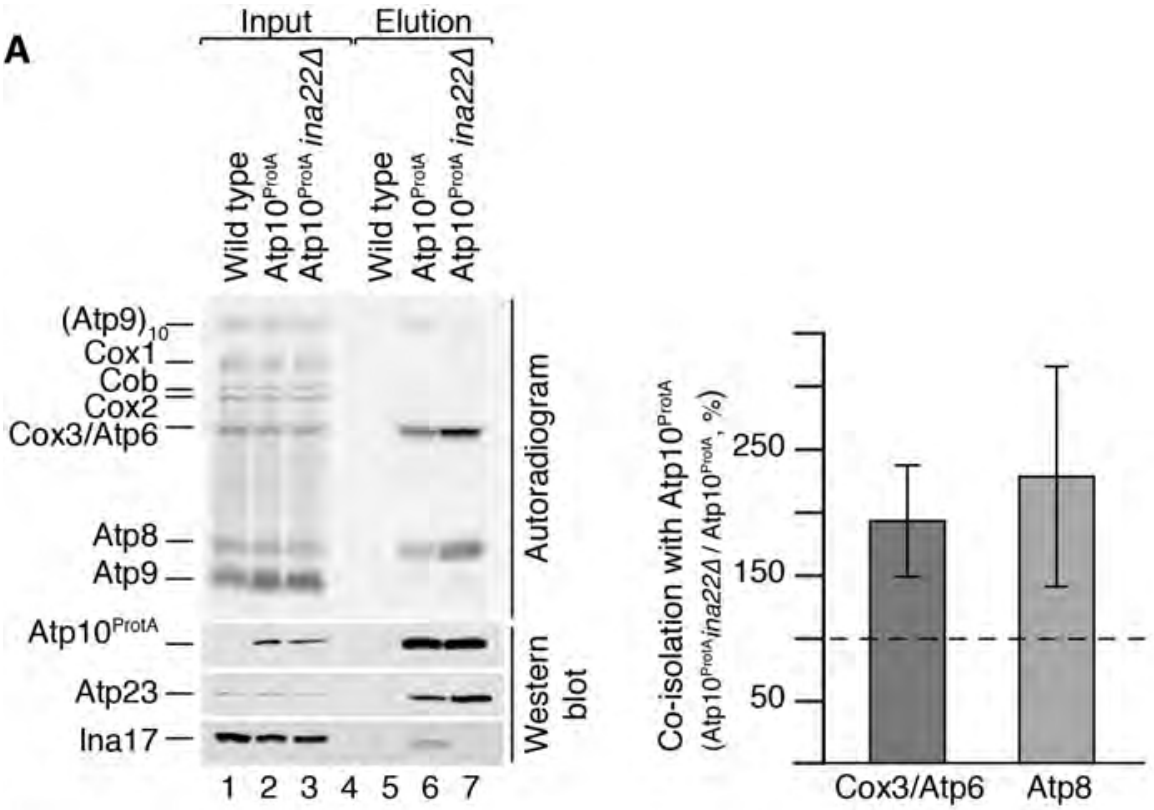

B
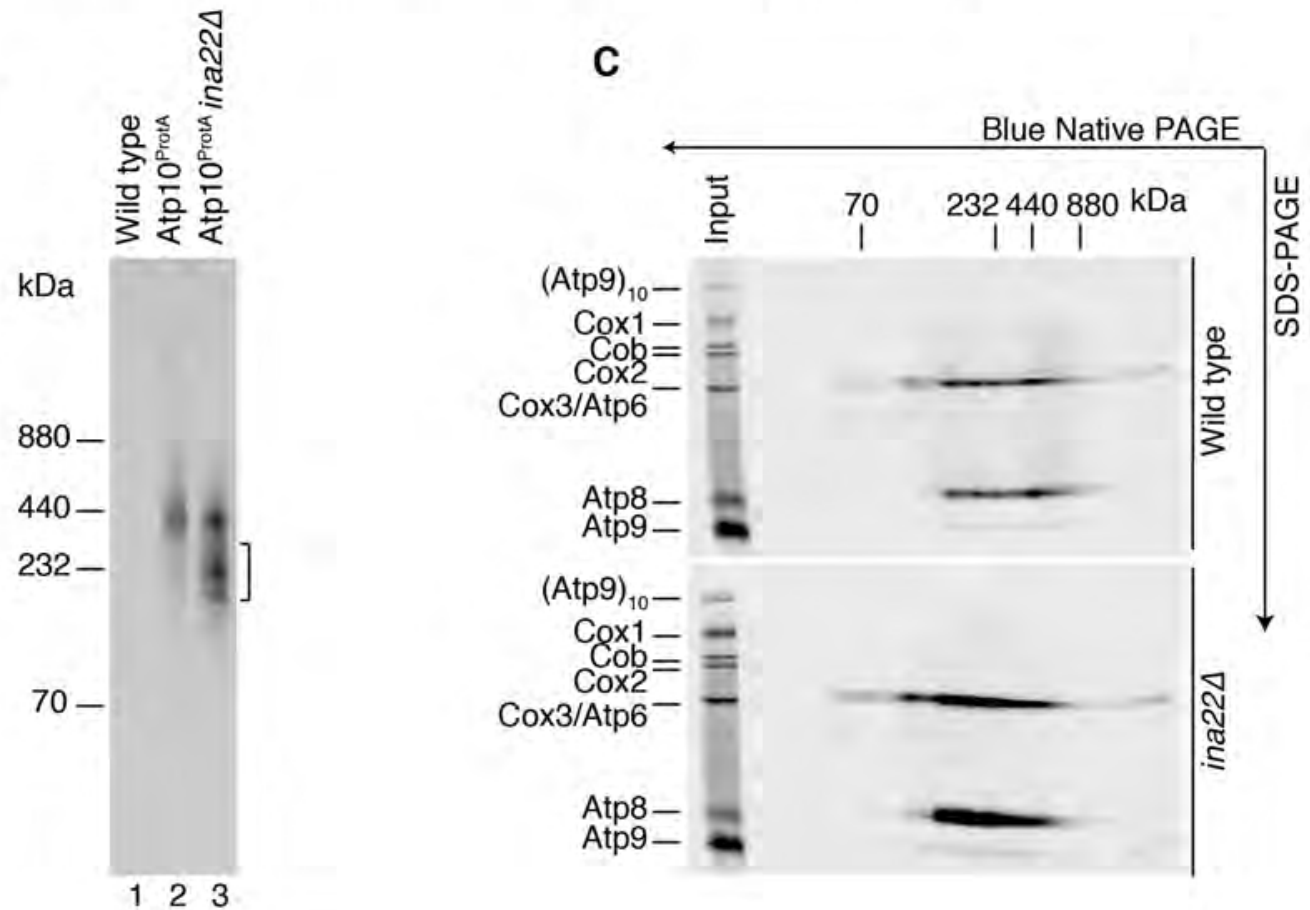

Figure 2.15 Atp10 interaction with Atp6 and Atp8 is increased in ina22 $\Delta$. A. Chloramphenicol-treated wild type, Atp10 $10^{\text {ProtA }}$ and Atp $10^{\text {ProtA }}$ ina22 $\Delta$ mitochondria were subjected to in organello labeling for $20 \mathrm{~min}$ and IgG-affinity chromatography. Input and elution fractions were analyzed by SDS-PAGE and digital autoradiography or Western blotting. The amounts of Cox3/Atp6 and Atp8, isolated from Atp $10^{\text {ProtA }}$ mitochondria, were set to $100 \%$. The levels of these proteins, isolated from Atp $10^{\text {ProtA }}$ ina22 $\Delta$ mitochondria, were then quantified (right panel). Signals in the elution fraction were normalized to the amount of isolated Atp $10^{\text {ProtA }}$ and to the input fraction. Input $=1 \%$ of elution. $\mathrm{n}=3, \pm$ SEM. B. Experiment was performed as in A, bound protein complexes were eluted via TEV-protease cleavage and analyzed by BN-PAGE and digital autoradiography. C. Part of the elution fraction of the experiment presented in panel B was analyzed by BN-PAGE, second dimension SDS-PAGE and digital autoradiography. 
A

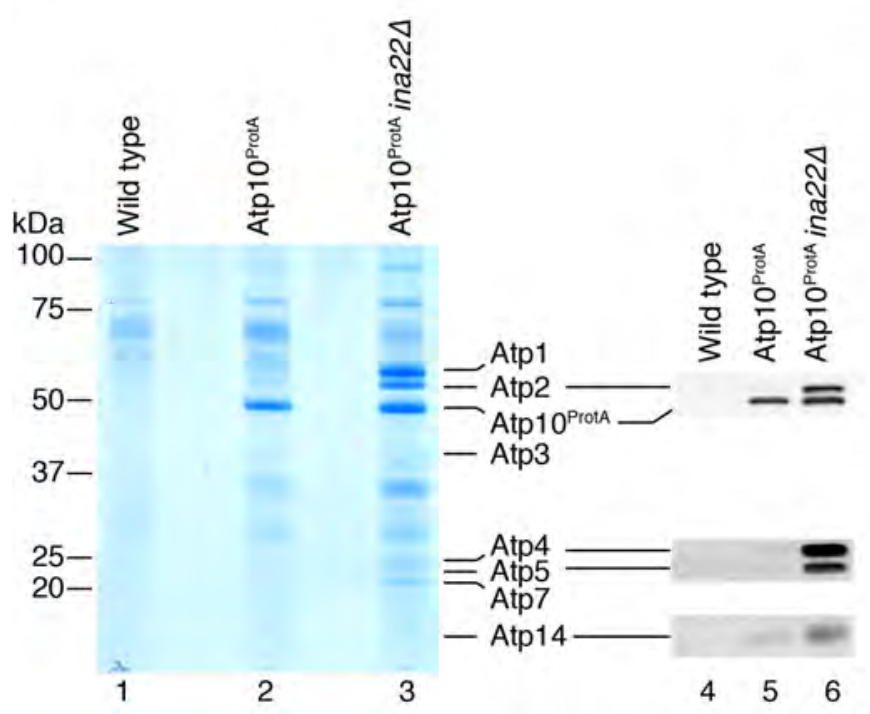

B

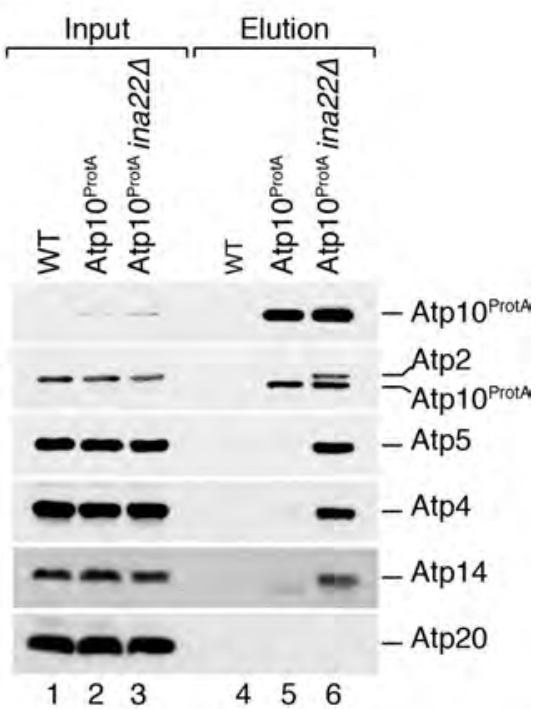

Figure 2.16 $\mathrm{F}_{1}$ and the peripheral stalk accumulate with Atp10 in ina22 $\Delta$. A. Wild type, Atp $10^{\text {ProtA }}$ and Atp $10^{\text {ProtA }}$ ina22 $\Delta$ mitochondria were subjected to IgG affinity chromatography. Ninety percent of the elution fraction was analyzed by SDS-PAGE and colloidal Coomassie staining (lanes 1-3). The remaining fraction was analyzed by SDS-PAGE and Western blotting with the indicated antibodies (lanes 4-6). B. Atp $10^{\text {ProtA }}$-containing protein complexes were purified via IgG affinity chromatography from Atp $10^{\text {ProtA }}$, Atp $10^{\text {ProtA }}$ ina22 $\Delta$ and wild type mitochondria. Input and elution fractions were analyzed by SDS-PAGE and Western blotting with the indicated antibodies.

analysis of these complexes by second dimension SDS-PAGE confirmed that they contained Atp6 and Atp8, but not Atp9 (Fig. 2.15C). Therefore, we confirmed that Atp6, Atp8, Atp23 and Atp10 form a complex together prior to association with the INA complex. Moreover, accumulation of this assembly intermediate in ina mutants points to an assembly stall.

\subsection{4 $\quad F_{1}$ and the peripheral stalk accumulate with Atp10 in ina22 $\Delta$}

Once the Atp6/Atp8 assembly intermediate is formed, it is competent to assemble with the peripheral stalk in order to form a large assembly module (Rak et al., 2011). As the INA complex physically interacts with peripheral stalk components and assembly of the peripheral stalk is severely impaired in ina mutants, it is the most likely candidate to mediate peripheral stalk assembly. The observed accumulation of the Atp10/Atp23/Atp6/Atp8-containing intermediate in ina22 $\Delta$ supports this hypothesis. Therefore, if the assumption is correct, peripheral stalk association with the Atp6/Atp8 intermediate should be reduced in ina22 $\Delta$.

To test whether this was the case, Atp10 interaction with nuclear-encoded complex $\mathrm{V}$ subunits was analyzed in wild type and ina22 $\Delta$ mitochondria. For this, Atp $10^{\text {ProtA }}$-containing protein complexes were isolated via IgG affinity chromatog- 
raphy and analyzed by SDS-PAGE and Western blotting. Surprisingly, already on a Coomassie stained SDS-PAGE, a prominent accumulation of various Atp10specific interaction partners was observed in ina22 2 (Fig. 2.16A, lanes 1-3). Further Western blot analysis confirmed an unexpected significant accumulation of nuclearencoded $\mathrm{F}_{1}$ and peripheral stalk subunits with Atp10 upon INA22 deletion (Fig. $2.16 \mathrm{~A}$, lanes $4-6)$. To verify that this prominent accumulation was not caused by altered steady-state levels of nuclear-encoded ATP synthase components, we performed a similar experiment to analyze elution fractions together with the input. Clearly, steady-state levels of $F_{1}$ and peripheral stalk components in ina22 $\Delta$ mitochondria were comparable to wild type, indicating that the observed findings are meaningful.

In conclusion, the data clearly indicate that $\mathrm{F}_{1}$ associates first with the Atp6/Atp8 module rather than with the Atp9 ring, as was assumed before.

\subsection{5 $\quad F_{1}$ and the peripheral stalk assemble with Atp6/Atp8 module.}

Previous data indicated that the Atp6/Atp8 assembly module accumulates with $\mathrm{F}_{1}$ and the peripheral stalk in ina22 $\Delta$. Thus, we asked whether they form a stable protein complex that could be isolated and analyzed. Therefore, Atp $10^{\text {ProtA }}$ containing complexes were isolated from WT and ina22 $\Delta$ mitochondria. Complexes were eluted via TEV-cleavage and analyzed by BN-PAGE and Western blotting with antibodies raised against Atp2, a component of $\mathrm{F}_{1}$, and Atp4, a component of the peripheral stalk. The obtained results indicate an extensive accumulation of Atp4containing protein complexes, as well as the Atp1-Atp2 dimer, a fully assembled $\mathrm{F}_{1}$ module and an Atp2-containing complex of around $232 \mathrm{kDa}$ upon INA22 deletion (Fig. 2.17A). Most likely, the $232 \mathrm{kDa}$ Atp2-containing complex represents a not fully assembled $F_{1}$, making it tempting to speculate that the Atp6/Atp8 module might serve as an assembly platform for $F_{1}$ and the peripheral stalk. However, the Atp2 and Atp4 containing complexes did not co-migrate on BN-PAGE. This implies that either two distinct Atp10-containing assembly intermediates exist, associated with the peripheral stalk or with $\mathrm{F}_{1}$, or that a single intermediate containing the peripheral stalk and $\mathrm{F}_{1}$ dissociates to smaller subcomplexes under BN-PAGE conditions.

To distinguish between these two scenarios, we performed a two-step affinity purification of these complexes. Firstly, Atp $10^{\text {ProtA }}$-containing assembly intermediates were natively isolated. Secondly, the obtained elution fractions were used for immunoprecipitation with either anti-Atp2 antibodies or a control pre-immune serum. The Western blot analysis of all elution fractions clearly showed that Atp2 remained associated with Atp4 and Atp5, two constituents of the peripheral stalk, within a single Atp10-associated protein complex (Fig. 2.17B).

Therefore, we concluded that a large assembly intermediate accumulates in ina 
A

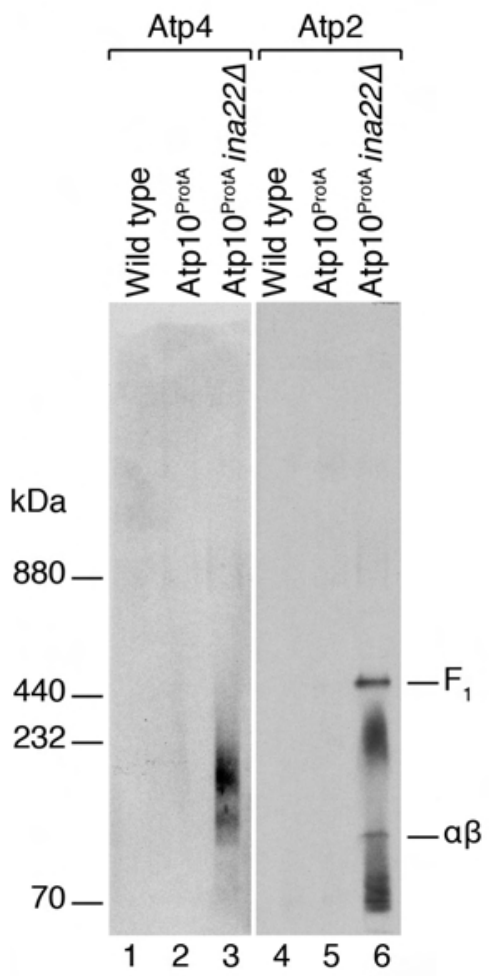

B

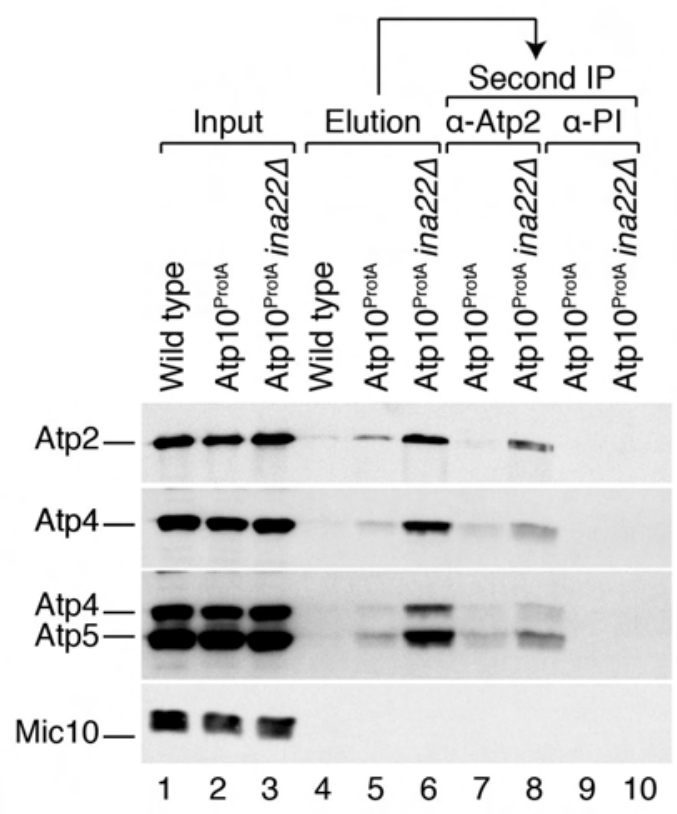

Figure 2.17 $\mathbf{F}_{1}$ and peripheral stalk assemble on Atp6/Atp8 module. A. Atp10 $0^{\text {ProtA }}$ containing protein complexes were purified via IgG affinity chromatography from Atp10 ${ }^{\text {ProtA }}$, Atp10 ${ }^{\text {ProtA }}$ ina22 $\Delta$ and wild type mitochondria. Purified complexes were eluted via TEV-protease cleavage and analyzed by BN-PAGE and Western blotting with the indicated antibodies. B. Wild type, Atp $10^{\operatorname{ProtA}}$ and $\operatorname{Atp} 10^{\operatorname{ProtA}}$ ina22 $\Delta$ mitochondria were subjected to IgG affinity chromatography. The obtained elution fractions were further subjected to immunoprecipitation (IP) with either anti-Atp2 antibodies or a control preimmune serum (PI). 1\% of the input fraction and all elution fractions were analyzed by SDS-PAGE and Western blotting with the indicated antibodies. $\alpha \beta$, a dimer of Atp1 and Atp2.

mutants, which contains an Atp6/Atp8 module, associated with Atp23 and Atp10, the peripheral stalk, and $F_{1}$. This intermediate is transient in wild type mitochondria and unstable during BN-PAGE analysis.

The currently proposed model of $\mathrm{F}_{1} \mathrm{~F}_{\mathrm{o}}$-ATP synthase assembly postulates that $\mathrm{F}_{1}$ associates with the Atp9 ring prior to association with the Atp6/Atp8/peripheral stalk module (Rak et al., 2011). However, our data clearly indicates that $\mathrm{F}_{1}$ assembles on the Atp6/Atp8 module, interacts with the peripheral stalk and therefore this association must take place prior to Atp9 ring binding. We noticed that for analyses of the Atp6/Atp8 module composition, Rak et al. (2011) used conditions, different from ours. In contrast to our analyses, much higher salt concentration and a higher $\mathrm{pH}$ were used by Rak et al. To assess if the high stringency buffer affected the integrity of the Atp6/Atp8 module, we purified Atp10 ${ }^{\text {ProtA }}$-containing complexes by IgG affinity chromatography under high-stringent and low-stringent conditions (see Materials and Methods), and analyzed them by SDS-PAGE and Western blot- 
A

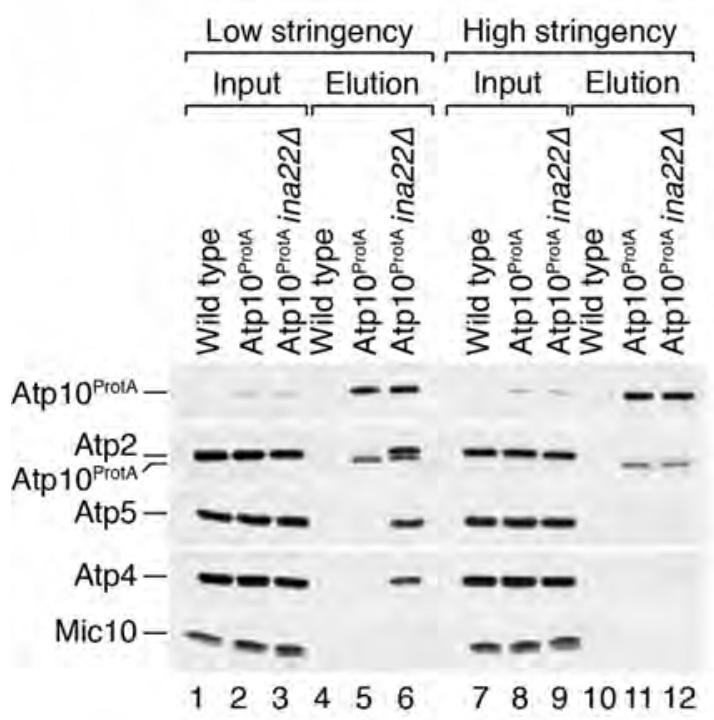

B
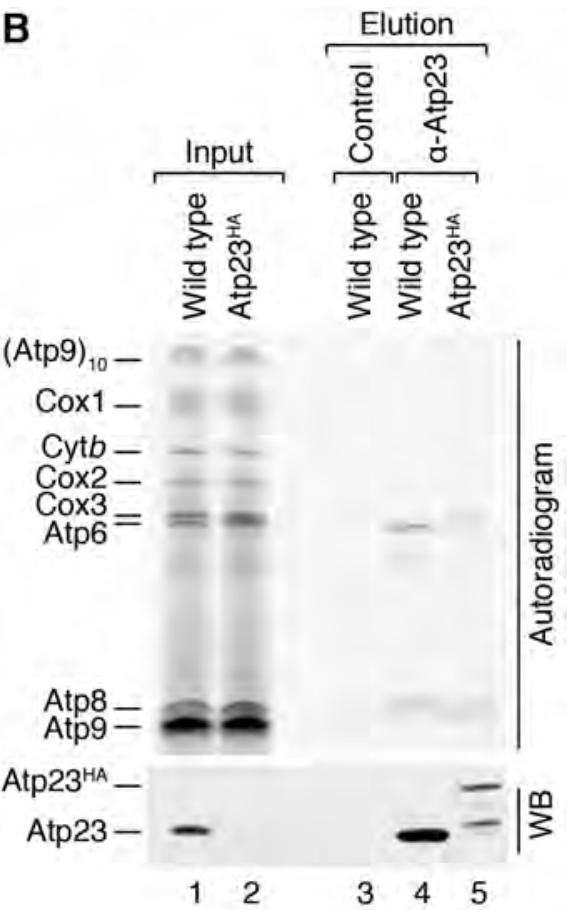

Figure 2.18 $\mathrm{F}_{1}$ and Atp23 associate with the Atp6/Atp8 module. A. Wild type, Atp $10^{\text {ProtA }}$ and Atp10 ${ }^{\operatorname{ProtA}}$ ina22 $\Delta$ mitochondria were solubilized under low- and high-stringent conditions and protein complexes were isolated via IgG affinity chromatography. The input and elution fractions were analyzed by SDS-PAGE and Western blotting with the indicated antibodies. Input $=1 \%$ of elution. B. Wild type and Atp $23^{\mathrm{HA}}$ mitochondria were subjected to in organello labeling and immunoprecipitation with control or anti-Atp23 antibodies, followed by SDS-PAGE and digital autoradiography analysis. Input $=2 \%$ of elution. WB, Western blotting.

ting (2.18A). As expected, $\mathrm{F}_{1}$ and peripheral stalk association with the Atp6/Atp8 module was drastically decreased under high-stringent conditions.

Our results indicate that Atp23 is a true constituent of the Atp6/Atp8 module, in contrast to what has been previously published (Rak et al., 2011). We noticed that for their experiments, Rak et al. used a C-terminally HA-tagged Atp23 variant. Therefore, to address whether a C-terminal tag interfered with the protein function, we performed an Atp23 immunoprecipitation after in organello labeling of mitochondrial gene products in wild type and Atp2 $3^{\mathrm{HA}}$ mitochondria. To our surprise, the C-terminal HA-tagging of Atp23 reduced protein stability and, subsequently, its association with newly-synthesized Atp6 and Atp8 (2.18B). Therefore, we conclude that Atp23 tagging interferes with Atp23 function.

\subsection{6 $\quad \mathrm{F}_{1} \mathrm{~F}_{\mathrm{o}}$-ATP synthase assembly is stalled in ina22 $\Delta$ prior to the last step}

To better understand how $\mathrm{F}_{1} \mathrm{~F}_{\mathrm{o}}$-ATP synthase assembly proceeds upon INA22 deletion, a second dimension analysis (2D) of the mitochondrial protein complexes was performed. For this, chloramphenicol-treated wild type and ina22 $\Delta$ mitochon- 
A

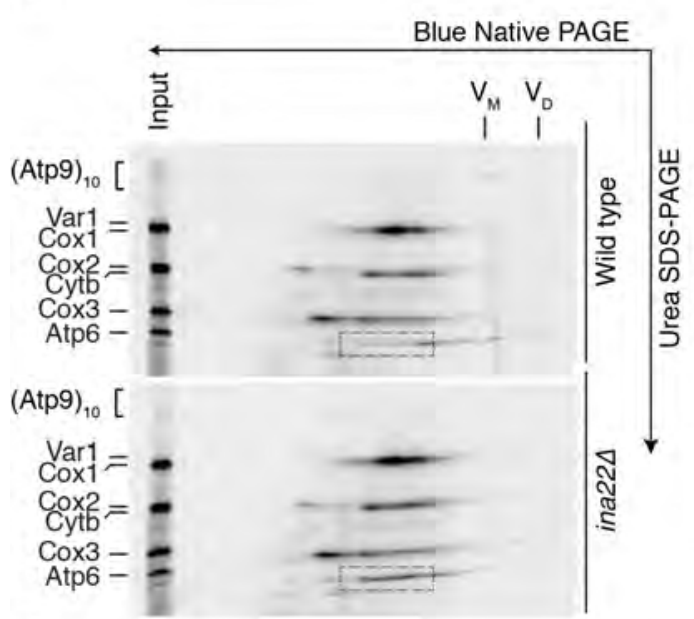

C

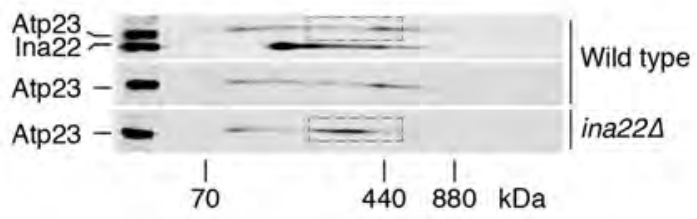

B

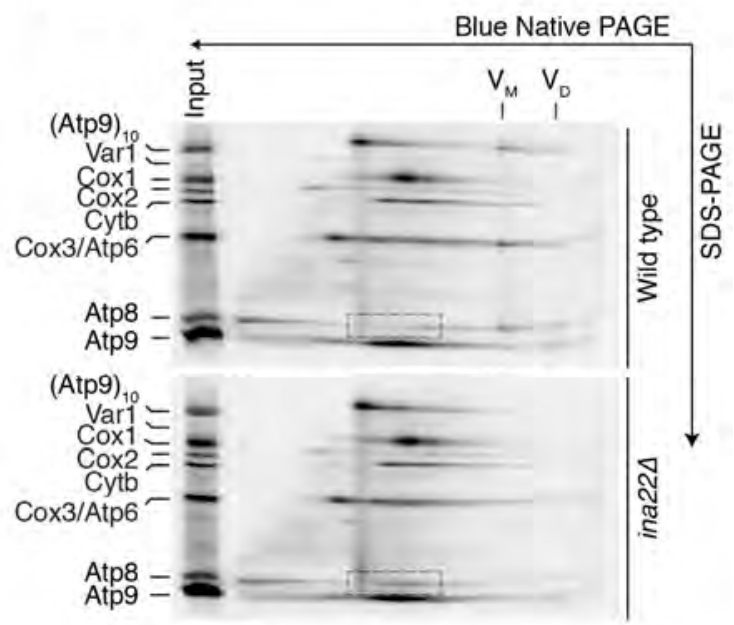

Figure 2.19 $\mathrm{F}_{1} \mathrm{~F}_{\mathrm{o}}$-ATP synthase assembly is stalled before the final step. A,B. Chloramphenicol-treated wild type and ina22 $\Delta$ mitochondria were subjected to in organello labeling for $15 \mathrm{~min}$. Protein complexes were analyzed by BN-PAGE and second dimension Urea SDS-PAGE (A) or SDS-PAGE (B) followed by digital autoradiography. Dashed boxes indicate accumulated Atp6/Atp8 assembly intermediates. B. Chloramphenicol-treated wild type and ina22 $\Delta$ mitochondria were analyzed by BN-PAGE, second dimension SDS-PAGE and Western blotting with the indicated antibodies. $\mathrm{V}_{\mathrm{M}}$, monomer of complex $\mathrm{V} ; \mathrm{V}_{\mathrm{D}}$, dimer of complex V. Dashed boxes indicate Atp23-containing protein complexes.

dria were subjected to in organello labeling for 15 minutes and protein complexes were separated by BN-PAGE in the first dimension and SDS-PAGE in the second dimension. It was previously shown that 15 minutes is sufficient time for mitochondrial-encoded complex $\mathrm{V}$ components to assemble into the mature enzyme (Rak et al., 2011). Moreover, in order to efficiently separate Atp6 from Cox3 and Atp8 from Atp9, two different gel systems were used for the second dimension protein separation, Urea SDS-PAGE and SDS-PAGE, respectively.

After 15 minutes, enough mitochondrial-encoded $\mathrm{F}_{1} \mathrm{~F}_{\mathrm{o}}$-ATP synthase components assembled into the mature enzyme, as Atp9, Atp6 and Atp8 were clearly incorporated into complex $\mathrm{V}$ monomers and dimers in wild type mitochondria (Fig. 2.19A and B, upper panels). However, the same was not observed in ina22 $\Delta$ mitochondria. No complex $\mathrm{V}$ monomers or dimers were formed after 15 minutes upon INA22 deletion. Moreover, an accumulation of a prominent assembly intermediate, containing Atp6 and Atp8 was observed in ina22 $($ Fig. 2.19A, B, lower panels). Further Western blot analysis of wild type and ina22 $\Delta$ mitochondria clearly indicated that Atp23 accumulates in the same complex as Atp6 and Atp8 (Fig. 2.19C) 
upon INA22 deletion. The latter is in agreement with all previously obtained data and points again to a stall in $\mathrm{F}_{1} \mathrm{~F}_{\mathrm{o}}$-ATP synthase assembly in the absence of Ina22.

\subsection{Ina22 associates with two major $\mathrm{F}_{1} \mathrm{~F}_{\mathrm{o}}$-ATP synthase as- sembly intermediates to link them together}

\subsubsection{Ina22 interacts with newly translated mitochondrial-encoded pro- teins}

All previously obtained data indicate that the Atp6/Atp8 module of the $\mathrm{F}_{1} \mathrm{~F}_{\mathrm{o}^{-}}$ ATP synthase accumulates upon INA22 deletion due to its impaired assembly into mature complex V. Moreover, our results point to a novel INAC interaction with Atp10 and Atp23, the Atp6 and Atp8 assembly factors. Therefore, we hypothesized that Ina22 association with Atp10 and Atp23 should take place within the Atp6/Atp8 module. Thus, INAC should interact with newly translated Atp6 and Atp8.

In order to verify Ina22 interaction with newly synthesized Atp6 and Atp8, Ina22 co-immunoprecipitation of radiolabeled mitochondrial-encoded proteins from control and chloramphenicol-treated mitochondria was performed (Fig. 2.20). The results showed that Ina22 associates with Atp6 and Atp8, as well as Atp9. Importantly, small amounts of these proteins were co-isolated with Ina22 from a nontreated sample, whereas their interaction was significantly increased upon translational activation. This indicates that Ina22 interacts with $\mathrm{F}_{1} \mathrm{~F}_{\mathrm{o}}$-ATP synthase assembly intermediates. Most interesting is that Ina22 association with the Atp9 ring was detected. From previously performed experiments it is known that neither Atp10 nor Atp23 stably associates with the Atp9 ring. Therefore, this finding allowed us to speculate that INAC might also be involved in the latest $\mathrm{F}_{1} \mathrm{~F}_{\mathrm{o}}$-ATP synthase assembly step, namely Atp9 ring connection. Taken together, these data confirm that Ina22 interacts with two major $\mathrm{F}_{1} \mathrm{~F}_{\mathrm{o}}$-ATP synthase assembly intermediates.

Moreover, it was shown that Ina22 also interacts with cytochrome $b$, a structural subunit of the cytochrome $b c_{1}$ complex (Fig 2.20). Taking a previously published SILAC analysis of the Ina22 $2^{\text {ProtA }}$ interactome into consideration, where Cbp proteins were identified as Ina22 interaction partners (Lytovchenko et al., 2014), we did not exclude that INAC might be involved in the biogenesis of complex III of the respiratory chain and investigated this further (see section 2.6).

\subsubsection{Ina22 interaction with $\mathrm{F}_{1} \mathrm{~F}_{\mathrm{o}}$-ATP synthase is Ina17-independent}

The INA complex has two constituents, namely Ina22 and Ina17. It was previously shown that the Ina17 interaction with $\mathrm{F}_{1} \mathrm{~F}_{\mathrm{o}}$-ATP synthase is Ina22-dependent 


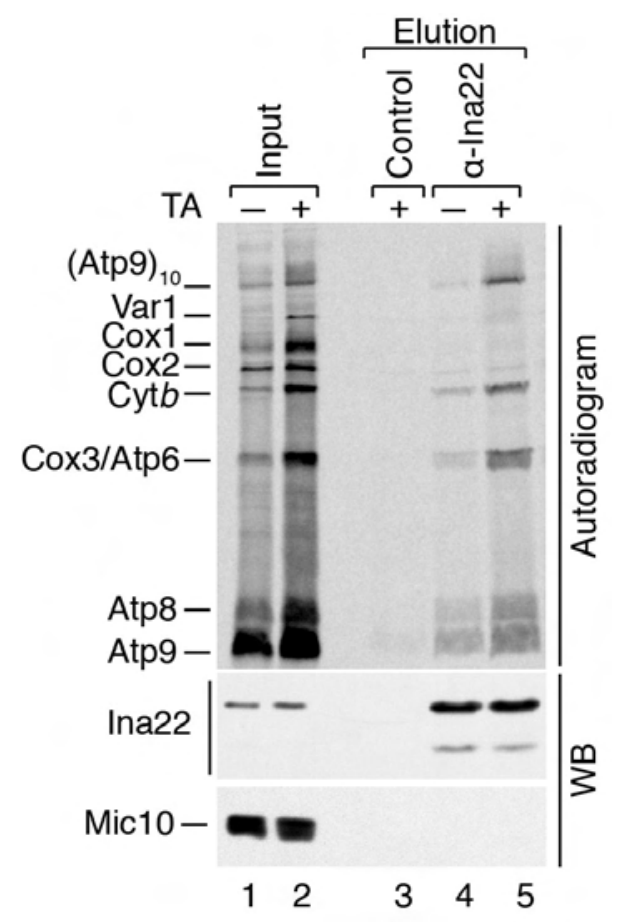

Figure 2.20 Ina22 interacts with newly translated mitochondrial-encoded proteins. Control and chloramphenicol-treated wild type mitochondria were subjected to in organello labeling for 20 minutes, followed by immunoprecipitation with either Ina22 antibodies or pre-immune serum. Isolated proteins were analyzed by SDS-PAGE and digital autoradiography. Input $=1 \%$ of elution. TA, translational activation. WB, Western blotting.

(Lytovchenko et al., 2014). However, it was not investigated whether Ina22 interacts with complex $\mathrm{V}$ components in an Ina17-dependent manner. To address this, we performed Ina22 immunoprecipitation from ina17 $\Delta$ mitochondria. Unfortunately, Ina22 becomes highly unstable upon INA17 deletion, which makes the results difficult to interpret (data not shown). To overcome this, we overexpressed INA22 in ina17A genetic background to restore Ina22 protein amounts. Importantly, overexpressed Ina22 was still imported into mitochondria (Fig. 2.21B, lanes 1-2). Next, to address Ina22 association with newly translated Atp6, Atp8 and Atp9, we radiolabeled mitochondrial-encoded proteins in wild type and Ina22个ina1 ${ }^{7} \Delta \mathrm{mi}$ tochondria and performed Ina22 immunoprecipitation. Surprisingly, a significant (around two-fold) increase of Ina22 association with Atp6 and Atp8 was detected, whereas its interaction with Atp9 remained unchanged (Fig. 2.21A). As levels of all co-isolated proteins were normalized to the amounts of Ina22 in the elution and input fractions, we excluded that this resulted from elevated Ina22 levels upon overexpression. Moreover, a Western blot analysis of Ina22 immunoprecipitation from wild type and Ina22个ina17 $\Delta$ mitochondria showed that Ina22 can still interact with nuclear-encoded complex V subunits, namely Atp2, upon INA17 deletion (Fig. 2.21B). Therefore we concluded that although Ina22 undergoes degradation in the absence of Ina17, the remaining Ina22 protein is still able to associate with $\mathrm{F}_{1} \mathrm{~F}_{\mathrm{o}^{-}}$ 
A
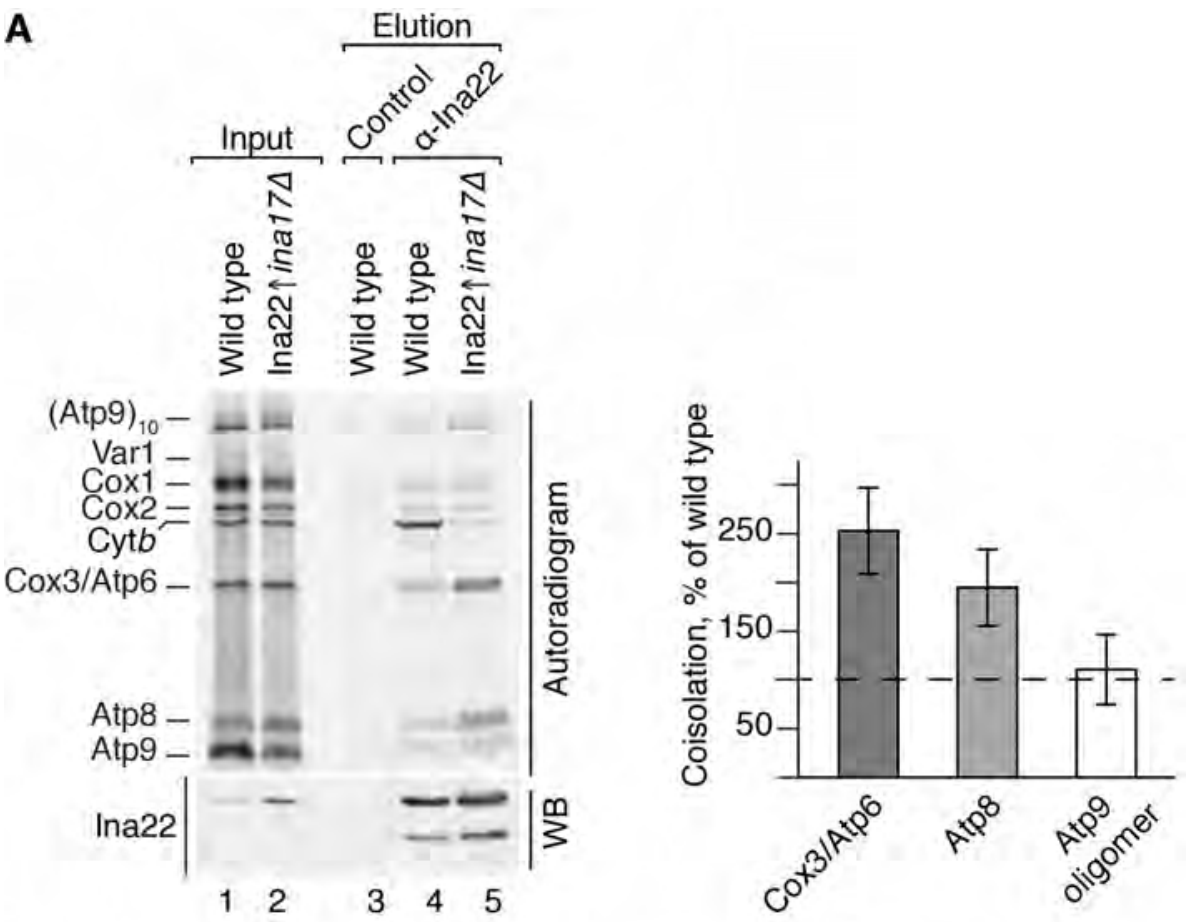

B

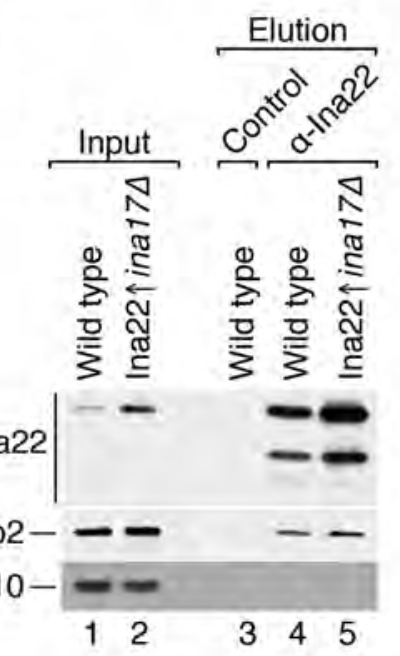

Figure 2.21 Ina22 interaction with the $F_{1} F_{0}$-ATP synthase is Ina17-independent. A. Mitochondrial-encoded proteins in wild type and Ina22个ina17 7 mitochondria were radiolabeled in organello for 20 min and co-immunoprecipitated with control or antiIna22 antibodies. Input and elution fractions were analyzed by SDS-PAGE, digital autoradiography and Western blotting (WB). For quantification, Atp6/Cox3, Atp8 and Atp9 signals in the elution fractions were normalized to isolated Ina22 and to the corresponding signals in the input fraction. The results represent the percentage of protein amounts co-isolated from Ina22个ina17 $\Delta$ mitochondria relative to wild type mitochondria (100\%). Input $=1 \%$ of elution. $\mathrm{n}=3, \pm$ SEM. B. Proteins from wild type and Ina $22 \uparrow$ ina $17 \Delta$ mitochondria were co-immunoprecipitated with anti-Ina22 antibodies. Input and elution fractions were analyzed by SDS-PAGE and Western blotting with the indicated antibodies. Input $=1 \%$ of elution. 
ATP synthase in order to facilitate its assembly. The latter result is in agreement with previous data, showing a less severe complex V assembly defect upon INA17 deletion, compared to INA22 deletion. Furthermore, there is no Atp6 processing defect upon INA17 deletion. Therefore, we conclude that Ina17 functions as an Ina22 chaperone and the observed $\mathrm{F}_{1} \mathrm{~F}_{\mathrm{o}}$-ATP synthase assembly defect in ina17 $\Delta$ is a secondary effect from decreased Ina22 levels.

\subsubsection{Ina22 interacts with the Atp9 ring and the Atp6/Atp8 module}

In contrast to the previously published assumption that the Atp9 ring associates with $\mathrm{F}_{1}$ in order to form a single assembly intermediate (Rak et al., 2011), our data indicates that $F_{1}$ rather associates with the Atp6/Atp8 module. Therefore, we shed a new light on the order of $\mathrm{F}_{1} \mathrm{~F}_{\mathrm{o}}$-ATP synthase assembly. However, the question of which assembly factors mediate the last assembly step remain open.

Up to now, none of the known assembly factors involved in the Atp6/Atp8 module biogenesis were shown to associate with the Atp9 ring and therefore to possibly mediate the last $\mathrm{F}_{1} \mathrm{~F}_{\mathrm{o}}$-ATP synthase assembly step. However, our data indicate that Ina22 interacts with both the newly translated Atp9 ring and Atp6/Atp8. Therefore, in order to show that INAC might mediate Atp9 ring assembly with the Atp6/Atp8 module, it was necessary to address if Ina22 interacts with the Atp9 ring and with Atp6/Atp8 within two distinct assembly intermediates.

A

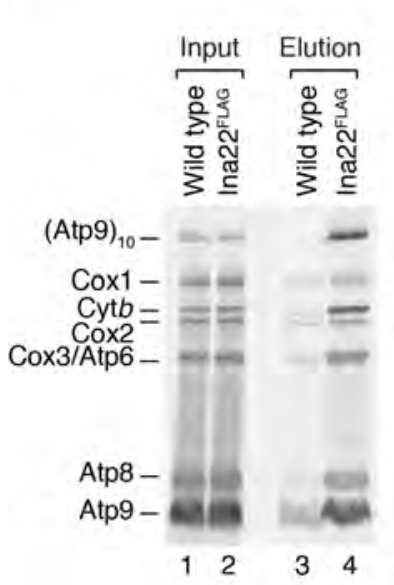

B

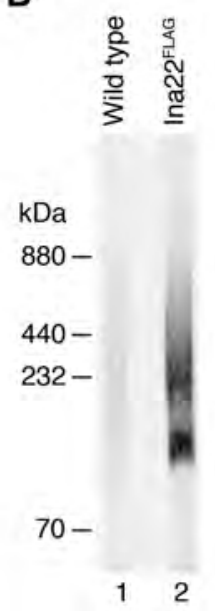

C

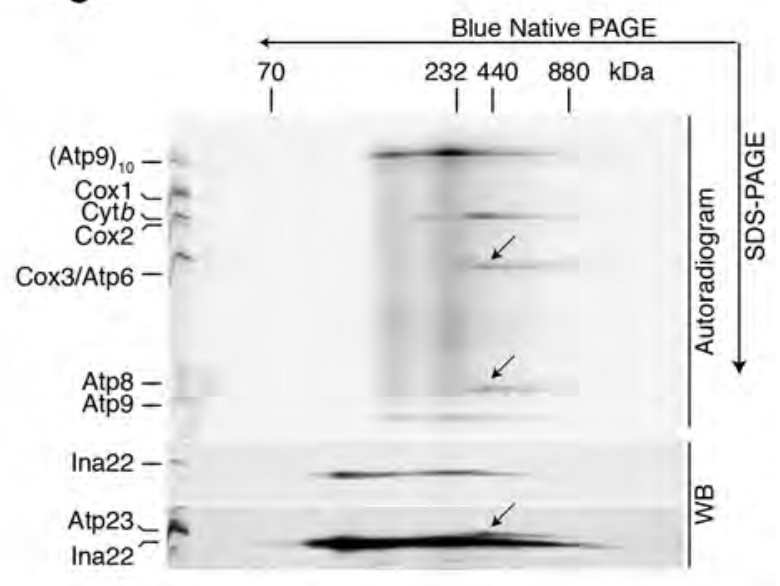

Figure 2.22 Ina22 interacts with the Atp9 ring and the Atp6/Atp8 module. A. Chloramphenicol-treated wild type and Ina22 ${ }^{\mathrm{FLAG}}$ mitochondria were subjected to in organello labeling for $20 \mathrm{~min}$, anti-FLAG affinity chromatography and analysis by SDS-PAGE and digital autoradiography. Input $=1 \%$ of elution. B. Chloramphenicol-treated wild type and Ina22 ${ }^{\mathrm{FLAG}}$ mitochondria were subjected to in organello labeling, anti-FLAG affinity chromatography and further analysis by BN-PAGE and digital autoradiography. C. Part of the Ina22 ${ }^{\mathrm{FLAG}}$ elution fraction of the experiment presented in panel B was subjected to BN-PAGE, second dimension SDS-PAGE, digital autoradiography and Western Blotting (WB). Arrows indicate the Atp6/Atp8 module. 
Therefore, we performed a native isolation of Ina22-containing protein complexes. To do so, Ina22 was tagged with a C-terminal FLAG-tag that allows a very efficient isolation of protein complexes. To verify whether a FLAG-tag affects Ina22 association with newly translated $\mathrm{F}_{1} \mathrm{~F}_{\mathrm{o}}$ - ATP synthase components, wild type and Ina $22^{\text {FLAG }}$ mitochondria were subjected to in organello labeling, followed by Ina $22^{\text {FLAG }}$ affinity purification. Importantly, the FLAG-tag did not alter Ina22 association with mitochondrial-encoded complex V subunits, despite the increased unspecific background binding (Fig. 2.22A). Next, we performed a similar Ina22 $2^{\text {FLAG }}$ isolation and eluted the isolated protein complexes natively with FLAG peptide. BN-PAGE analysis of the elution fractions showed that Ina22 associates with several assembly intermediates (Fig. 2.22B), however, it was not possible to distinguish which mitochondrial-encoded proteins were constituents of these complexes. Therefore, we analyzed elution fractions by second dimension SDS-PAGE (2D). Strikingly, a $2 \mathrm{D}$ analysis revealed that two major $\mathrm{F}_{1} \mathrm{~F}_{\mathrm{o}}$-ATP synthase assembly intermediates were co-isolated with Ina $22^{\mathrm{FLAG}}$, one of them contained the Atp9 ring, and another one contained Atp6, Atp8 and Atp23 (Fig. 2.22C). Surprisingly, more Atp9 ring was co-purified with Ina22 compared to the amounts of co-purified Atp6 and Atp8. The latter implies that INAC interaction with the Atp6/Atp8 module might indeed occur only transiently, as seen before (Fig. 2.12), and that Ina22 association with the Atp9 ring is more stable.

In conclusion, we established that Ina22 interacts with two main $\mathrm{F}_{1} \mathrm{~F}_{\mathrm{o}}$-ATP synthase assembly intermediates and therefore is the most likely candidate to mediate their assembly.

\subsubsection{Ina22 can be cross-linked to the Atp9 ring and the peripheral stalk}

In the previous section, it was shown that Ina22 is present in two main $\mathrm{F}_{1} \mathrm{~F}_{\mathrm{o}^{-}}$ ATP synthase assembly intermediates. To establish whether Ina22 interacts with newly translated $\mathrm{F}_{1} \mathrm{~F}_{\mathrm{o}}$-ATP synthase components directly, we performed in organello chemical crosslinking after radiolabeling of mitochondrial-encoded proteins in wild type and Ina $22^{\text {FLAG }}$ mitochondria and isolated Ina22 ${ }^{\text {FLAG }}$ under denaturing conditions. As a result, Atp9 was crosslinked to Ina $22^{\text {FLAG }}$, confirming their direct interaction (Fig. 2.23A). Surprisingly, no Ina22 crosslink was obtained to either Atp6 or Atp8 under the same conditions. This implies that either the chosen crosslinker was not suitable for Ina22 crosslinking to Atp6 or Atp8, or that their interaction is mediated by other proteins, for example peripheral stalk components. To test this, we tried to crosslink Ina22 to Atp4, a membrane-embedded component of the peripheral stalk. Indeed, we successfully obtained an Ina22 crosslink to Atp4, implying that the Ina22-Atp6/Atp8 interaction might indeed be mediated by the peripheral stalk. (Fig. 2.23B).

In summary, Ina22 directly associates with the Atp9 ring and components of 


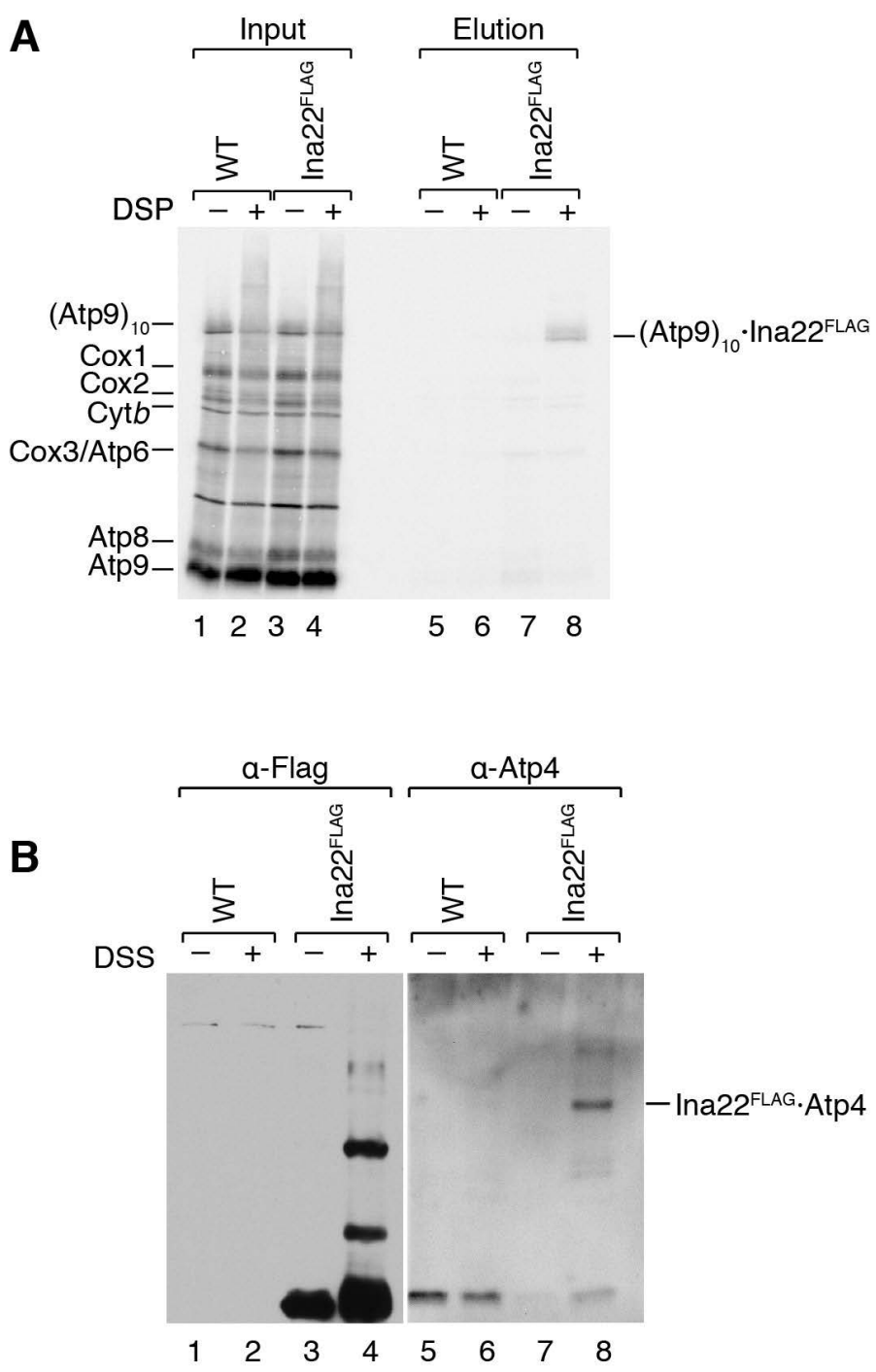

Figure 2.23 Ina22 can be crosslinked to Atp9 ring and Atp4. A. Wild type and Ina22 $2^{\text {FLAG }}$ mitochondria were subjected to chemical crosslinking with DSP and Ina22 ${ }^{\mathrm{FLAG}}$ was purified under denaturing conditions. Input and elution fractions were analyzed by SDS-PAGE and digital autoradiography. B. Wild type and Ina22 $2^{\text {FLAG }}$ mitochondria were subjected to chemical crosslinking with DSS and Ina22 $2^{\mathrm{FLAG}}$ was purified under denaturing conditions. Elution fractions were analyzed by SDS-PAGE and Western blotting with anti-FLAG or anti-Atp4 antibodies.

the peripheral stalk to link two assembly intermediates in the final assembly step.

\subsection{INAC function in assembly of cytochrome $b c_{1}$ complex}

\subsubsection{The INA complex interacts with Cbp proteins}

Previously, Ina $22^{\text {ProtA }}$ protein complexes were purified via IgG affinity chromatography and analyzed by quantitative mass spectrometry after Stable Isotope Labeling with Amino acids in Cell culture (SILAC) (Lytovchenko et al., 2014). Aside from nuclear-encoded subunits of $\mathrm{F}_{1} \mathrm{~F}_{\mathrm{o}}$-ATP synthase, three cytochrome $b$ assem- 
bly factors were identified, namely Cbp3, Cbp6 and Cpb4. These findings point to a putative INAC role in the assembly of complex III of the respiratory chain, also called the cytochrome $b c_{1}$ complex. However, further analysis of ina mutants did not reveal any defect in mature complex III in either ina22 $\Delta$ or ina17 $\Delta$ mitochondria and therefore the role of INAC association with Cbps was not investigated further.

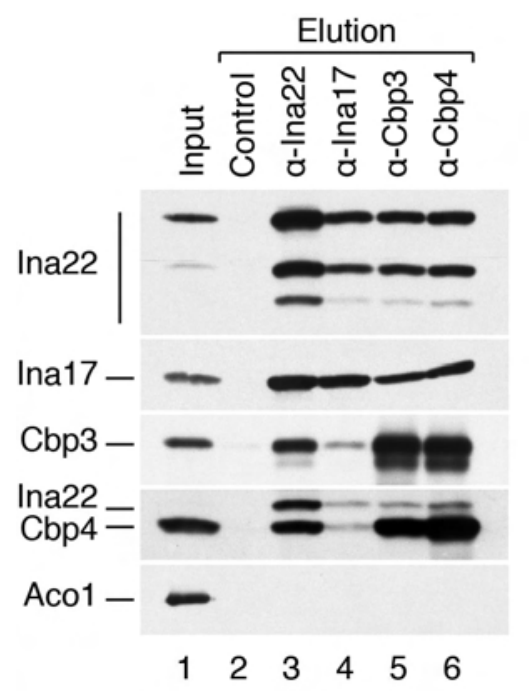

Figure 2.24 The INA complex interacts with Cbp proteins. Wild type mitochondria were solubilized and subjected to immunoprecipitation with control, anti-Ina22, antiIna17, anti-Cbp3 and anti-Cbp4 antibodies. Results were analyzed by SDS-PAGE and Western blotting with the indicated antibodies. Input $=1 \%$ of elution.

Our recently obtained data, however, revealed that Ina22 interacts with newlytranslated cytochrome $b$ (Fig 2.20). Therefore we were interested in having a closer look at INAC association with the complex III assembly machinery.

First of all, we confirmed INAC interaction with Cbps by immunoprecipitating endogenous proteins with antibodies raised against Ina22, Ina17, Cbp3 and Cbp4. Analysis of elution fractions by SDS-PAGE and immunoblotting confirmed that the INA complex indeed associates with the cytochrome $b$ assembly machinery (Fig. 2.24). Interestingly, significantly less Cbp3 and Cbp4 were co-isolated with Ina17 compared to Ina22, but approximately the same amounts of Ina22 and Ina17 were co-isolated with Cbp3 and Cbp4. Therefore, we concluded that antibodies, raised against the matrix domain of Ina17, do not bind efficiently to Ina17 associated with Cbp proteins, as the matrix domain might be involved in these interactions. To conclude, the endogenous INA complex physically associates with early cytochrome $b$ assembly machinery.

\subsubsection{INAC interacts with Cbps in a cytochrome $b$-dependent manner}

Cytochrome $b(\mathrm{Cyt} b)$ is the only mitochondrial-encoded component of the respiratory chain complex III. Its expression in mitochondria is controlled by various 
nuclear-encoded factors, one of those being Cbp1. Cbp1 interacts with the 5'-end of $C O B$ mRNA to regulate its stability (Dieckmann et al., 1982; Islas-Osuna et al., 2002). Upon $C B P 1$ deletion, $C O B$ mRNA becomes highly unstable and the mature protein is therefore not synthesized.

A
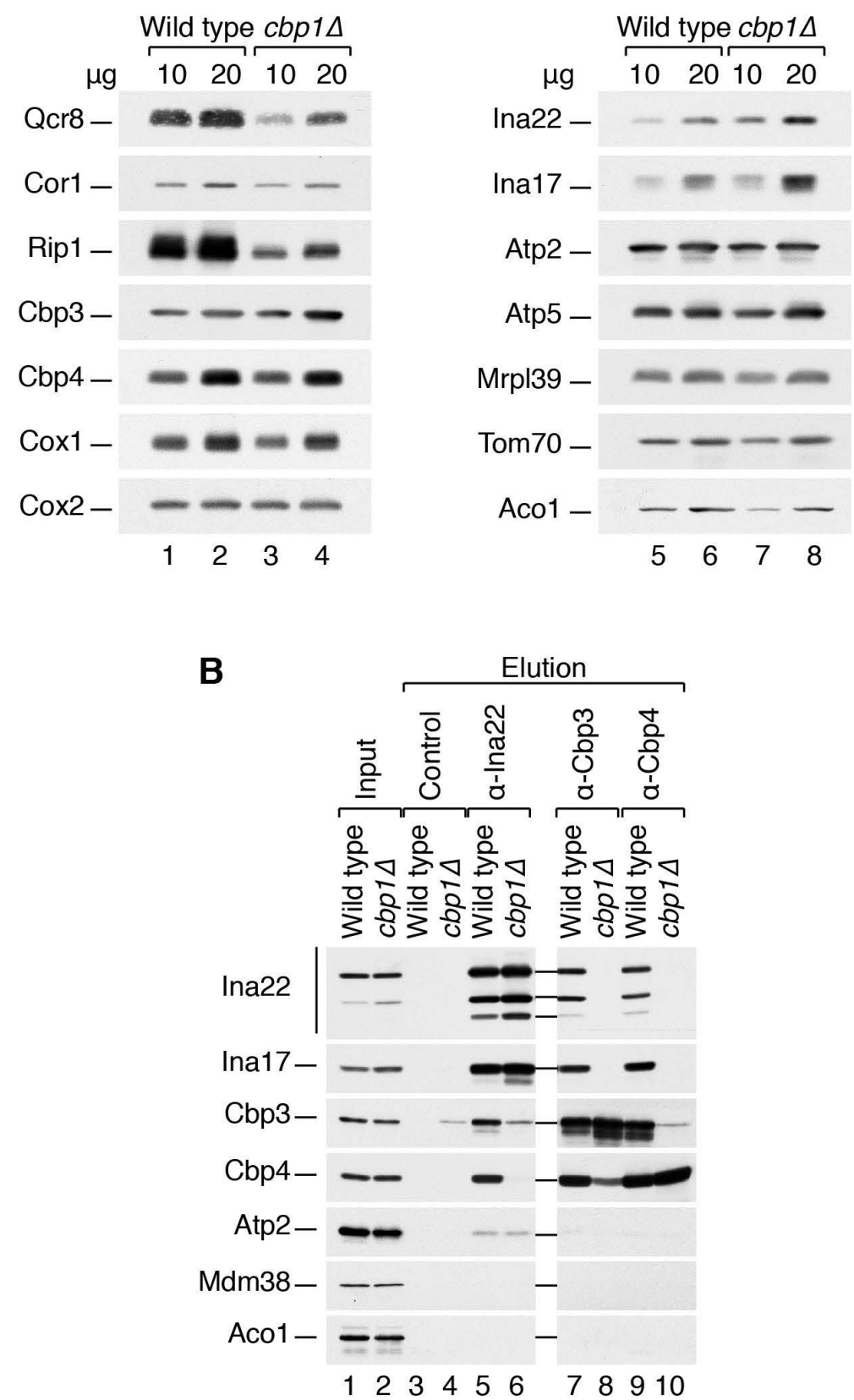

Figure 2.25 The Ina22 interaction with Cbps is cytb-dependent. A. Wild type and mutant mitochondria were solubilized and analyzed by SDS-PAGE and Western blotting with the indicated antibodies. B. Wild type and $\operatorname{cbp} 1 \Delta$ mitochondria were solubilized and subjected to immunoprecipitation. Input and elution fractions were analyzed by SDS-PAGE and Western blotting with the indicated antibodies. Input $=1 \%$ of elution.

To test whether INAC interaction with Cbps requires cytochrome $b$, a yeast 
strain lacking the CBP1 gene was generated in an intronless background. Mitochondria from control wild type and $\operatorname{cbp} 1 \Delta$ strains were isolated and analyzed by SDS-PAGE and Western blotting. CBP1 deletion resulted in downregulation of complex III, since less complex III structural components (Qcr8, Rip1, Cor1) were present in mutant mitochondria compared to wild type (Fig. 2.25A). However, CBP1 deletion did not alter levels of Cbp3 and Cbp4, as well as structural subunits of $\mathrm{F}_{1} \mathrm{~F}_{\mathrm{o}}$-ATP synthase. Surprisingly, a slight upregulation of Ina22 and Ina17 was observed in $\operatorname{cbp} 1 \Delta$ mitochondria.

Next, we used wild type and $c b p 1 \Delta$ mitochondria for immunoprecipitations with control, anti-Ina22, anti-Cbp3 and anti-Cbp4 antibodies. Analysis of the elution fractions by SDS-PAGE and Western blotting showed that CBP1 deletion, and the subsequent absence of cytochrome $b$, completely abolishes the Ina22 interaction with Cbp proteins. Importantly, the absence of cytochrome $b$ did not change Ina22 association with either Ina17 or Atp5, but significantly decreased Cbp3-Cbp4 interaction, in agreement with previously published data (Gruschke et al., 2011) (Fig. $2.25 \mathrm{~B})$.

To conclude, Ina22 interacts with Cbp proteins in a cytochrome $b$-dependent manner.

\subsubsection{A C-terminal protein A tag does not alter the function of Cbp proteins}

For isolation of native Cbp-containing protein complexes, Cbp3, Cbp4 and Cbp6 proteins were tagged with a Protein A tag at their C-terminus. To analyze if Cbp tagging affected complex III biogenesis, we checked the growth behavior of the generated strains on non-fermentable carbon sources. Growth of yeast expressing tagged Cbp proteins was comparable to the growth of a wild type strain on both fermentable (YPD) and non-fermentable (YPG) media at all temperatures tested (Fig. 2.26A). Moreover, to make sure that cytochrome $b$ translation was not affected, mitochondrial-encoded proteins in wild type, $\mathrm{Cbp} 3^{\text {ProtA }}, \mathrm{Cbp} 4^{\text {ProtA }}$ and $\mathrm{Cbp} 6^{\text {ProtA }}$ strains were pulse-labeled with $\left[{ }^{35} \mathrm{~S}\right]$ methionine and analyzed by SDS-PAGE and autoradiography. No cytochrome $b$ biogenesis defect was detected (Fig. 2.26B) and therefore we concluded that tagged Cbp proteins remain functional.

\subsubsection{INAC interacts with Cbps in a discrete protein complex}

Though we confirmed that tagged Cbp proteins were functional in cytochrome $b$ biogenesis, their ability to interact with the INA complex had not been shown. Therefore, we subjected wild type, $\mathrm{Cbp} 3^{\text {ProtA }}, \mathrm{Cbp} 4^{\text {ProtA }}$ and $\mathrm{Cbp} 6^{\text {ProtA }}$ mitochondria to IgG affinity chromatography and analyzed their association with INAC and other components of complex III by Western blotting. Qcr8, an early structural sub- 
A

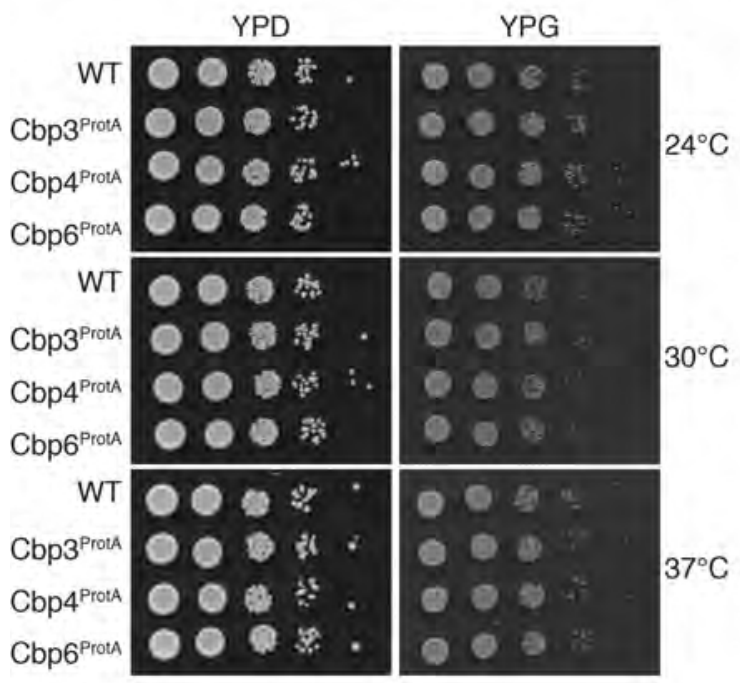

B

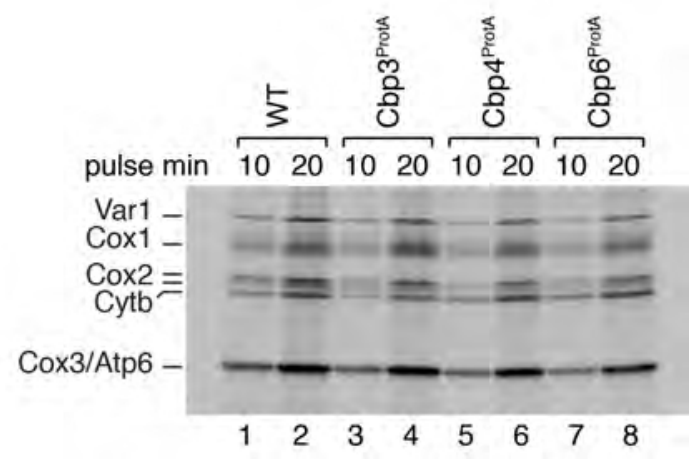

Figure 2.26 C-terminal protein A tag does not alter the function of Cbp proteins. A. A serial dilution of yeast cells was spotted on fermentable (YPD) or non fermentable (YPG) media and grown at the indicated temperatures for 2-5 days. WT, wild type. B. Mitochondrial-encoded proteins in the indicated strains were pulse-labeled with $\left[{ }^{35} \mathrm{~S}\right]$ methionine and analyzed by SDS-PAGE and autoradiography. WT, wild type.

unit of complex III, was co-isolated only with Cbp4, in agreement with published data (Gruschke et al., 2012) (Fig. 2.27A). Moreover, Cor1, a late complex III structural subunit, was not co-isolated with any of the Cbp proteins, also in agreement with previous work (Gruschke et al., 2012). Importantly, neither of the tested complex V structural subunits (Atp2, Atp3, Atp5, Atp10) were co-purified with Cbps in sufficient amounts for detection by Western blotting. Therefore, we confirmed that Cbp proteins do not physically associate with components of the $\mathrm{F}_{1} \mathrm{~F}_{\mathrm{o}}$-ATP synthase. However, both Ina17 and Ina22 were co-isolated with $\mathrm{Cbp} 3^{\text {ProtA }}$, Cbp4 ${ }^{\text {ProtA }}$ and $\mathrm{Cbp} 6{ }^{\text {ProtA }}$. It is worth mentioning that less Ina17 and Ina22 were isolated with Cbp4 compared to Cbp3 and Cbp6. Therefore, we do not exclude that the Protein A tag on Cbp4 might affect this association.

To address if INAC interacts with Cbps in a distinct protein complex, we subjected wild type, $\mathrm{Cbp} 3^{\text {ProtA }}, \mathrm{Cbp} 4^{\text {ProtA }}$ and $\mathrm{Cbp} 6^{\text {ProtA }}$ mitochondria to $\operatorname{IgG}$ affinity chromatography and eluted bound protein complexes natively via protease cleavage. Analysis by BN-PAGE and Western blotting with antibodies raised against Ina17 and Ina22 revealed a single protein complex of approximately $350 \mathrm{kDa}$, containing Ina17 and Ina22, that co-purified with Cbp3, Cbp4 and Cbp6 (Fig. 2.27B). Lesser amounts of this complex were isolated via Cbp4, implying again that either less Cbp4 is present or Protein A tagged Cbp4 does not associate with the complex efficiently.

To summarize, Cbp proteins interact with INAC in a distinct protein complex, which does not contain structural subunits of $\mathrm{F}_{1} \mathrm{~F}_{\mathrm{o}}$-ATP synthase. 
A
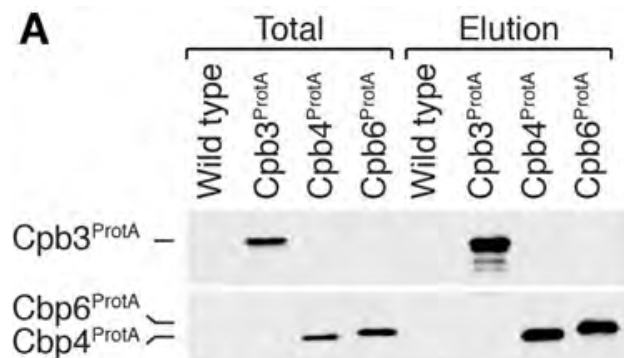

$$
\text { Qcr8 - }
$$

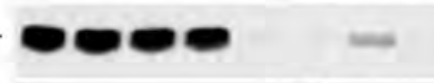

Cor1 -

Ina22

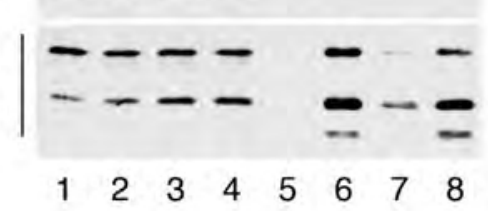

B

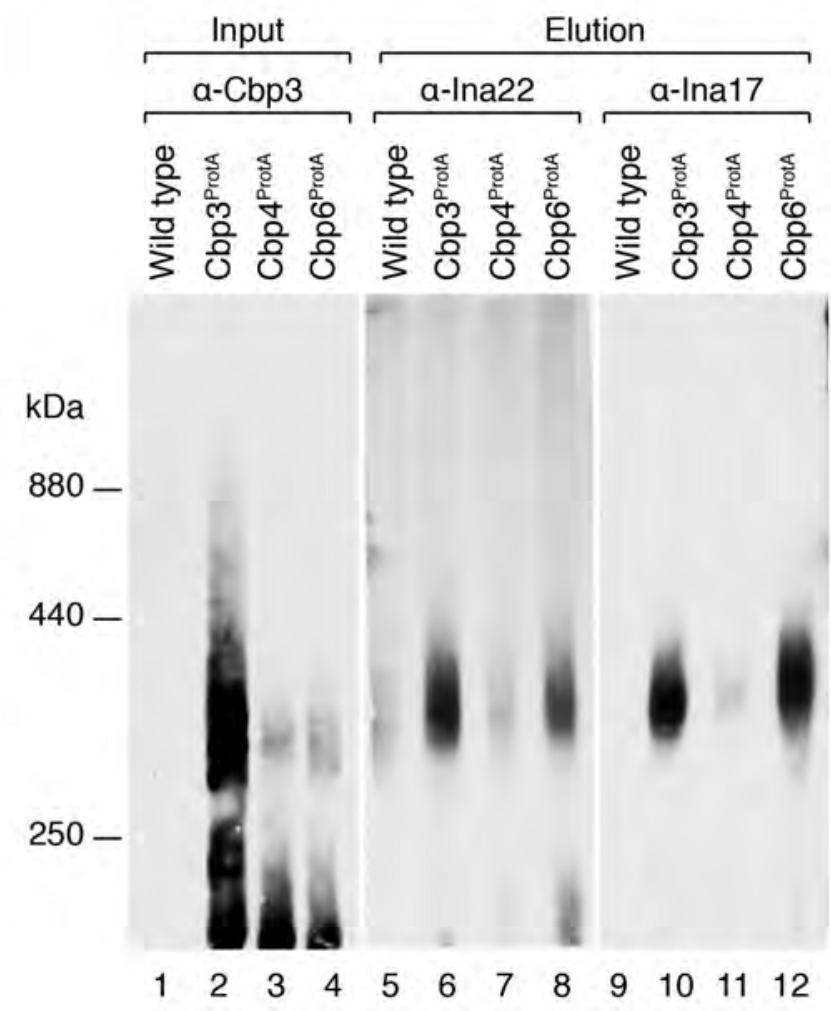

Figure 2.27 INAC-Cbps containing protein complex can be natively isolated. A. Wild type, $\mathrm{Cbp} 3^{\text {ProtA }}, \mathrm{Cbp} 4^{\text {ProtA }}$ and $\mathrm{Cbp} 6^{\text {ProtA }}$ mitochondria were subjected to $\operatorname{IgG}$ affinity chromatography. Bound protein complexes were eluted with low $\mathrm{pH}$ and analyzed by SDS-PAGE and Western blotting with the indicated antibodies. Input $=2 \%$ of elution. B. Wild type, $\mathrm{Cbp} 3^{\text {ProtA }}, \mathrm{Cbp} 4^{\text {ProtA }}$ and $\mathrm{Cbp} 6^{\text {ProtA }}$ mitochondria were subjected to IgG affinity chromatography and bound protein complexes were eluted natively via TEV protease cleavage. Elution fractions were analyzed by BNPAGE and Western blotting with the indicated antibodies.

\subsubsection{Newly translated cytochrome $b$ is present in INAC*CBP complex}

Our previously obtained data indicate that INAC interaction with Cbps requires the presence of cytochrome $b$. Therefore, we wondered if the newly identified com- 
A

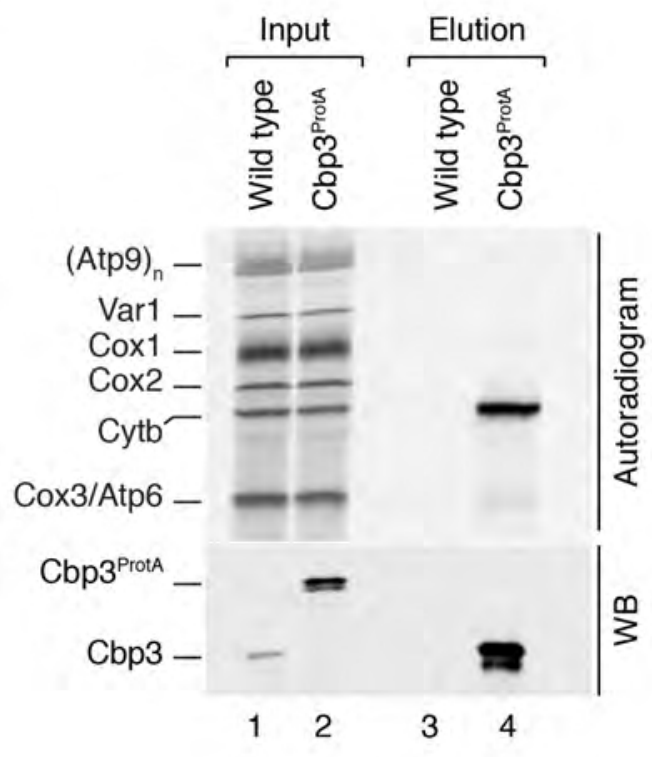

B
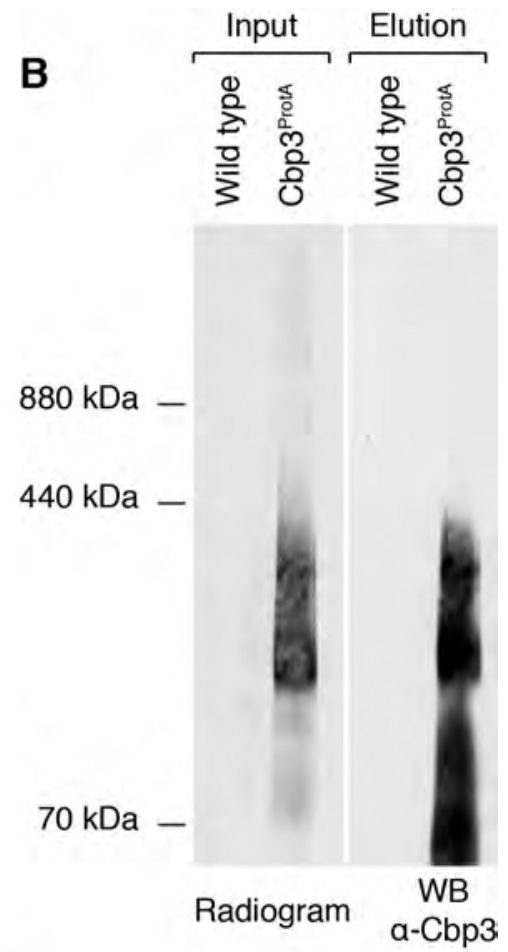

C

Blue Native PAGE

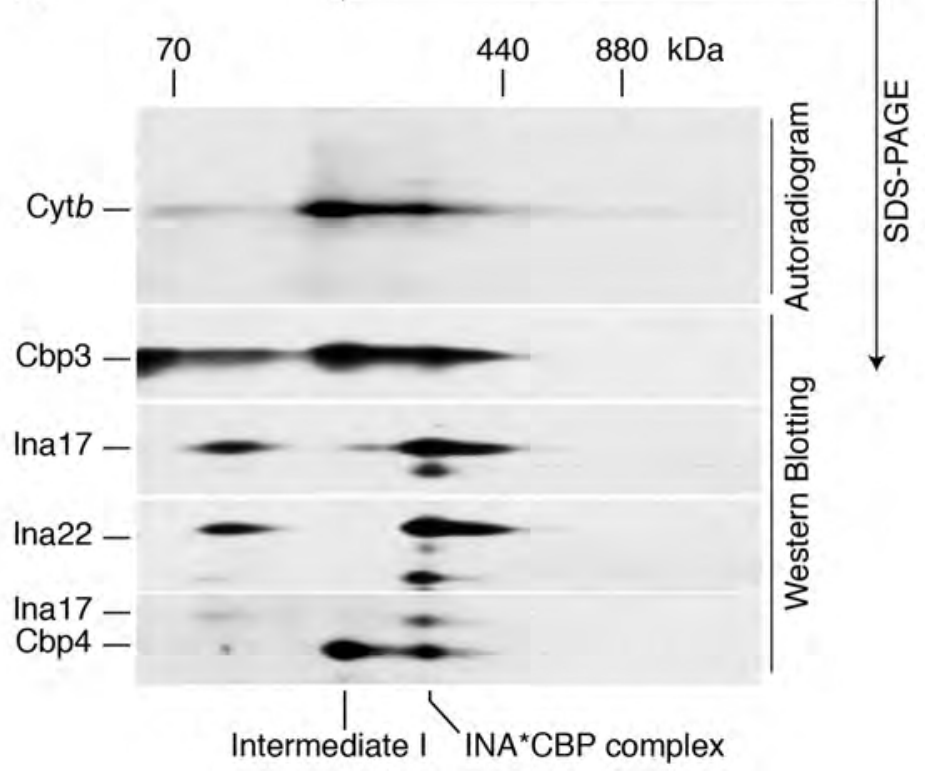

Figure 2.28 Newly translated cytochrome $b$ is present in the INAC-CBP complex. A. Wild type and $\mathrm{Cbp} 3^{\mathrm{ProtA}}$ mitochondria were subjected to in organello labeling and IgG affinity chromatography. Bound protein complexes were eluted natively via TEV protease cleavage and analyzed by SDS-PAGE and digital autoradiography or Western blotting with anti-Cbp3 antibodies. Input $=1 \%$ of elution. WB, Western blot. B. Part of the elution of the experiment presented in panel A was analyzed by BN-PAGE and either Western blotting (WB) with anti-Cbp3 antibodies, or autoradiography. C. Part of the elution of the experiment presented in panel A was analyzed by BN-PAGE, second dimension SDS-PAGE, Western blotting and digital autoradiography. 
plex, containing Cbp proteins together with Ina17 and Ina22, includes newly translated cytochrome $b$. Thus, we subjected wild type and $\mathrm{Cbp} 3^{\text {ProtA }}$ mitochondria to in organello labeling and IgG affinity chromatography. Bound protein complexes were eluted natively via TEV protease cleavage and further analyzed. SDS-PAGE analysis, followed by autoradiography confirmed that the Protein A tag was efficiently cleaved and sufficient amounts of cytochrome $b$ were co-isolated with $\mathrm{Cbp} 3^{\text {ProtA }}$ (Fig. 2.28A). Next, we analyzed eluted protein complexes by BN-PAGE. As can be seen from the results, several cytochrome $b$-containing complexes were isolated with Cbp3, which most probably represent early complex III assembly intermediates (Fig. 2.28B). Based on previously reported data, it is known that Cbp3 associates with newly translated cytochrome $b$ while associated with ribosomes and within assembly intermediate I (Gruschke et al., 2012). However, when another gradient of BN-PAGE was used, it was possible to clearly separate two Cbp3-cytochrome $b$ containing protein complexes. Next, to analyze components of the isolated complexes in more detail, we separated the Cbp3 $3^{\text {ProtA }}$ elution fraction by BN-PAGE and second dimension SDS-PAGE. A digital autoradiography analysis confirmed the presence of two distinct co-isolated cytochrome $b$ - containing complexes (Fig. 2.28C). The first complex, of smaller size, contained cytochrome b, Cbp3 and Cbp4 and likely represents intermediate I of complex III assembly. A second complex, of a larger size additionally contains Ina17 and Ina22 and therefore represents a previously unidentified INAC*CBP macromolecular assembly. Moreover, we observed that Ina17 and Ina22 co-migrate with Cbp3 in a complex of approximately $100 \mathrm{kDa}$. However, as this complex did not contain newly translated cytochrome $b$, we assumed that it is most likely a degradation product of the larger complex.

Cbp3 was previously shown to stably associate with Cbp6, even upon solubilization in 1\% Triton (Gruschke et al., 2011), and therefore it is highly unlikely that a free Cbp6 pool exists in the cell. To address whether the identified INAC*CBP complex contains Cbp6, we analyzed protein complexes in wild type and cbp6 $\Delta$ mitochondria by second dimension SDS-PAGE and Western blotting. In intact wild type mitochondria, we observed that Cbp3 co-migrates with Ina17 and Ina22 in the newly identified INAC*CBP complex (Fig. 2.29). As expected, upon Cbp6 deletion, Cbp3 shifts from both INAC* ${ }^{*} \mathrm{BP}$ complex and intermediate I to a free pool. Importantly, Ina17 and Ina22 shift to lower molecular weight complexes as well.

Taken together, the data indicate that $\mathrm{Cbp} 6$ is also a component of the INAC*CBP complex and is required for its integrity.

\subsubsection{The INAC* CBP complex is destabilized upon complex III assem- bly stalling}

Assembly of complex III is a step-wise process. It starts with translation of cytochrome $b$ and its correct hemylation, and continues with the addition of nuclear- 


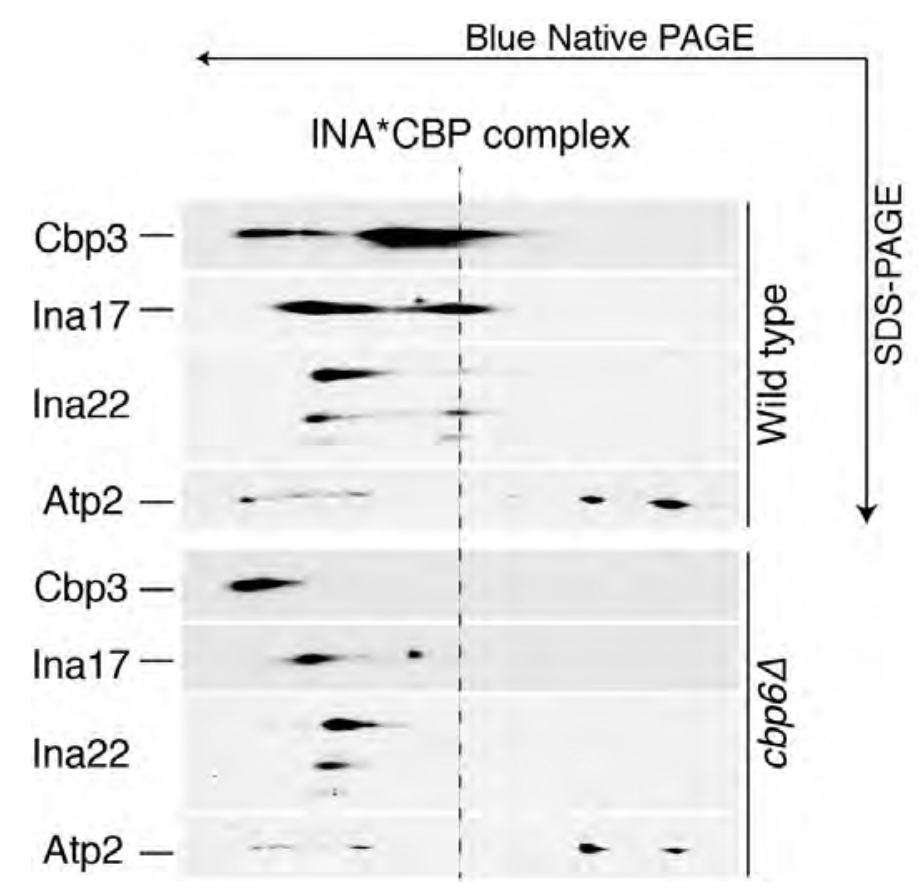

Figure 2.29 The INAC*CBP complex is unstable upon $C B P 6$ deletion. Wild type and cbp6 6 mitochondria were solubilized and protein complexes were analyzed by BNPAGE and second dimension SDS-PAGE, followed by Western blotting with the indicated antibodies.

encoded structural subunits (Gruschke et al., 2011; Gruschke et al., 2012). Importantly, Cbp3 and Cbp6 remain associated with cytochrome $b$ only during the initial steps of complex III assembly, before the first structural subunits, namely Qcr8 and Qcr7, are added. Deletion of QCR8 was shown to stall complex III assembly and to trap cytochrome b, Cbp3, Cbp6 and Cbp4 in the intermediate I (Gruschke et al., 2012). To analyze what happens to the INAC* ${ }^{*} B P$ complex upon $Q C R 8$ deletion and the accumulation of intermediate I, we isolated mitochondria from wild type and $q$ cr $8 \Delta$ strains and analyzed mitochondrial protein complexes by second dimension SDS-PAGE and Western blotting. As before, we detected a co-migration of INAC and Cbp3 protein within the INAC*CBP complex (Fig. 2.30). However, upon $Q C R 8$ deletion, Cbp3 from the INAC*CBP complex shifts to intermediate I, whereas INA proteins shift to lower molecular weight complexes. Therefore, we established that the CBP*INAC interaction indeed does not take place within complex III assembly intermediate I. Moreover, upon stalling of complex III assembly at early stages, the $\mathrm{INAC}^{*} \mathrm{CBP}$ complex is destabilized.

\subsubsection{INAC does not associate with known cytochrome $b$ translational activators}

Based on all previously collected data, it is clear that INAC associates only with cytochrome $b$ and its early assembly factors, but not with structural subunits of 


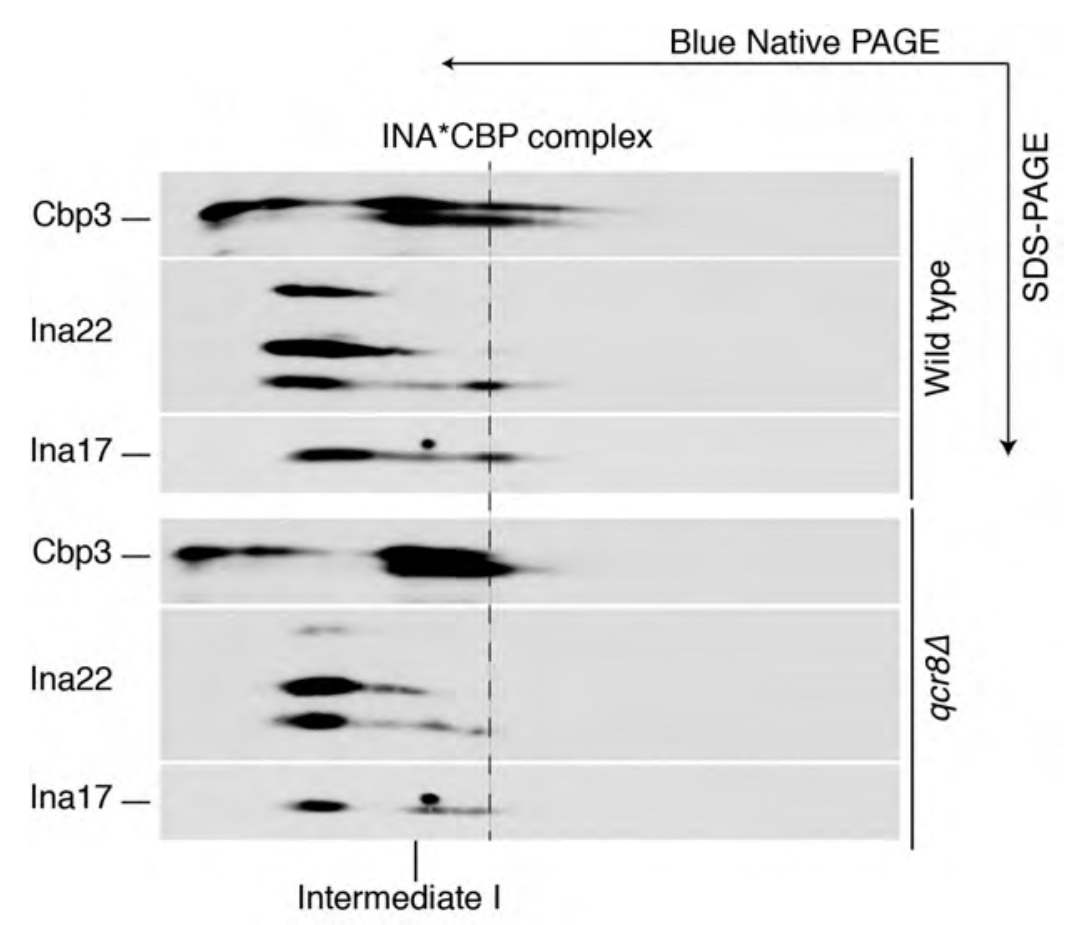

Figure 2.30 INAC* CBP complex is destabilized upon complex III assembly stalling. Wild type and $q c r 8 \Delta$ mitochondria were solubilized and protein complexes were analyzed by BN-PAGE and second dimension SDS-PAGE, followed by Western blotting with the indicated antibodies.

complex III. Therefore, we screened the literature for other proteins that are involved in the early biogenesis of complex III and found that translation of cytochrome $b$ is under the control of two ribosome-associated translational activators, Cbs1 and Cbs2 (Rödel, 1986). Though it was shown that Cbp proteins do not interact with Cbs1 and Cbs2 (Gruschke et al., 2011), we addressed whether an interaction between INAC and Cbs1 or Cbs2 takes place.

For this, Cbs1 and Cbs2 were genomically fused with a Protein A tag at their C-terminus. To exclude that tagging affected protein functionality, a growth test was performed for wild type, $\mathrm{Cbs}^{\text {ProtA }}$ and $\mathrm{Cbs} 2^{\text {ProtA }}$ strains on fermentable and non-fermentable carbon sources. Strains expressing tagged Cbs proteins grew comparable to wild type (Fig. 2.31A). Moreover, Western blot analysis of wild type, $\mathrm{Cbs}^{\text {ProtA }}$ and $\mathrm{Cbs} 2^{\text {ProtA }}$ mitochondria did not reveal any severe alterations in mitochondrial protein levels (Fig. 2.31B). Therefore, we concluded that the generated strains are functional.

To address the INAC-Cbs interaction, we subjected wild type, Cbs1 $1^{\text {ProtA }}$ and Cbs $2^{\text {ProtA }}$ mitochondria to IgG affinity chromatography and analyzed purified protein complexes by SDS-PAGE and Western blotting. Moreover, we used Cbp3 ${ }^{\text {ProtA }}$ mitochondria as a positive control for complex isolation. Whereas Ina17 and Ina22 were co-isolated with Cbp3, no Ina17 or Ina22 was detected in Cbs1 ProtA and Cbs2 $2^{\text {ProtA }}$ elutions. This finding confirms the absence of INAC interaction with 
A

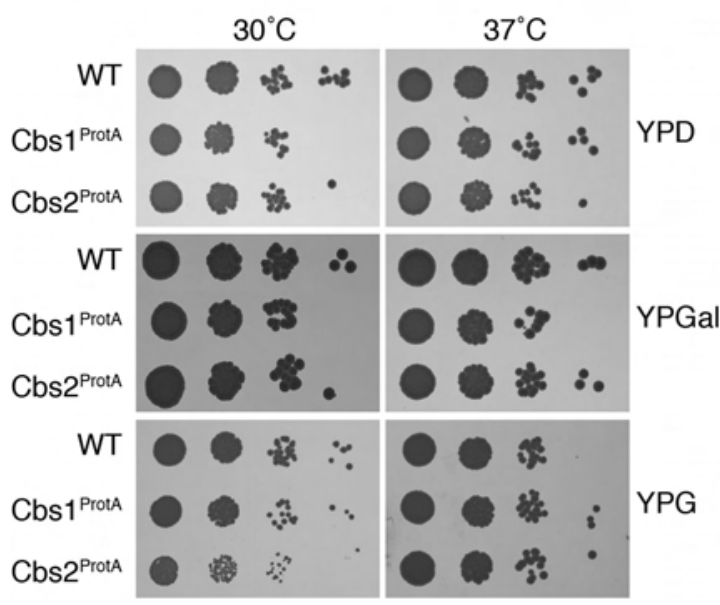

B

$\mu \mathrm{g}$
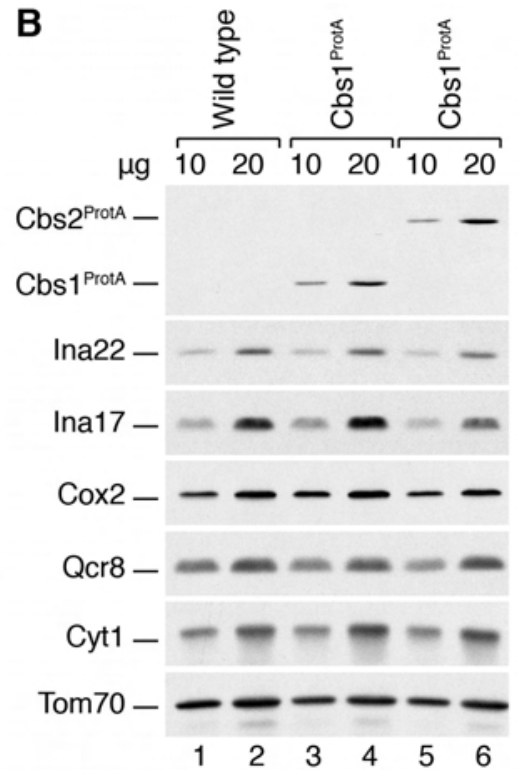

C
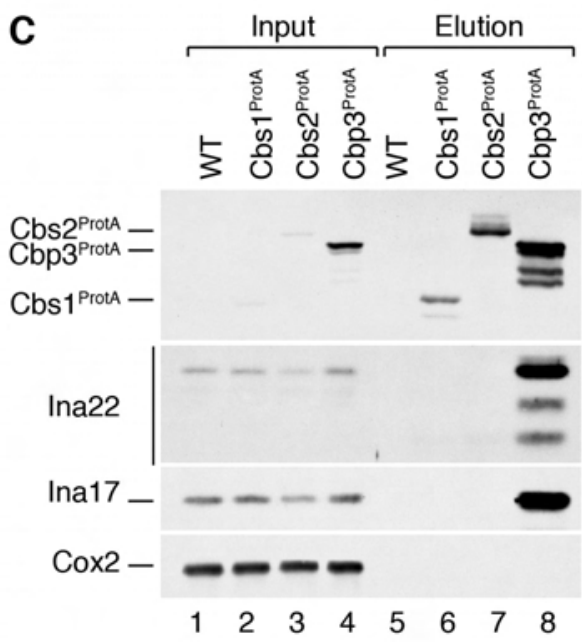

Figure 2.31 INAC does not associate with cytochrome $b$ translational activators Cbs1 and Cbs2. A. A serial dilution of yeast cells was spotted onto plates containing fermentable (YPD) or non-fermentable (YPG, YPGal) carbon source and grown at indicated temperatures for 2-5 days. B. Wild type, $\mathrm{Cbs}^{\text {ProtA }}$ and $\mathrm{Cbs}^{\text {ProtA }}$ mitochondria were solubilized and analyzed by SDS-PAGE and western blotting with the indicated antibodies. C. Wild type, Cbs1 $1^{\text {ProtA }}$ and $\mathrm{Cbs} 2^{\text {ProtA }}$ mitochondria were subjected to IgG affinity chromatography and the elution fractions were analyzed by SDS-PAGE and Western blotting with the indicated antibodies. Input $=1 \%$ of elution.

cytochrome $b$ translational activators and, therefore, excludes the possibility that the INA complex might be involved in translational regulation of cytochrome $b$.

\subsubsection{Proof of INAC* CBP interaction}

All previously performed experiments did not reveal a defect in complex III biogenesis upon deletion of INA complex components (Lytovchenko et al., 2014). 
The steady-state levels of complex III, as well as the activity of the mature enzyme in ina mutants, never differed from that of wild type mitochondria. Moreover, detailed analyses of $c b p$ mutants did not indicate any impairment of $\mathrm{F}_{1} \mathrm{~F}_{\mathrm{o}}$-ATP synthase (Gruschke et al., 2011; Gruschke et al., 2012). However, our data clearly show that the INA complex associates with Cbp3, Cbp6, Cbp4 and newly translated cytochrome $b$. The absence of a clear phenotype for either complex III or $\mathrm{V}$ in ina and $c b p$ mutants respectively makes the function of this association unclear.

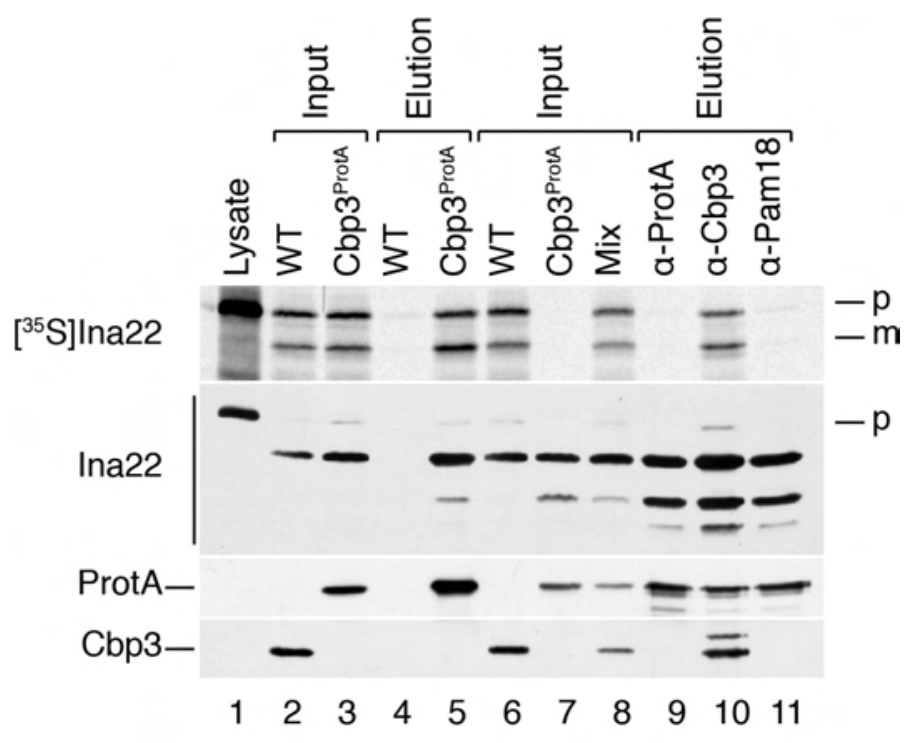

Figure 2.32 INAC interaction with Cbps is not a post-lysis artifact. $\left[{ }^{35} \mathrm{~S}\right] \mathrm{Ina} 22$ was imported into wild type and Cbp3 $3^{\text {ProtA }}$ mitochondria (lanes 6 and 7 ). Mitochondria were lysed separately and the two lysates were mixed (lane 8). Then, the mixed mitochondrial lysate was subjected to affinity chromatography with either anti-Cbp3 antibodies (lane 10), IgG coupled beads (lane 9) or control serum (lane 11) raised against Pam18. Samples were analyzed by SDS-PAGE, followed by autoradiography (upper panel) and Western blotting with the indicated antibodies (three lower panels). Please note that anti-Ina22 antibodies recognize radioactively labeled Ina22.

Therefore, we asked whether the observed interaction of the INA complex with Cbp proteins indeed happens in vivo or whether we observe an unspecific protein aggregation in post lysis mitochondria. To check if the latter was the case, the following experiment was performed. First, in vitro synthesized radioactively labeled Ina22 was imported into WT and $\mathrm{Cbp} 3^{\text {ProtA }}$ mitochondria and the mitochondria were subjected to IgG affinity chromatography. Clearly, imported Ina22 was co-isolated with Cbp3 (Fig. 2.32, lanes 1-5), showing that $\left[{ }^{35} \mathrm{~S}\right]$ Ina22 can be co-isolated with Cbp3 in principle. Next, $\left.{ }^{35} \mathrm{~S}\right]$ Ina22 was imported into wild type, but not into Cbp3 ${ }^{\text {ProtA }}$ mitochondria. After this, mitochondria were solubilized separately and the lysate of wild type mitochondria (with imported Ina22) was mixed with $\mathrm{Cbp}^{\text {ProtA }}$ lysate (with no radiolabeled Ina22). The obtained mixed lysate (Fig. 2.32, lane 8) was subjected to affinity chromatography, either with beads coupled to IgG (should isolate Cbp3 ${ }^{\text {ProtA }}$ ), antibodies against endogenous Cbp3 (should isolate both tagged and 
non-tagged Cbp3), or control serum ( $\alpha$-Pam18, would still react with Protein A). Elution fractions were analyzed by SDS-PAGE, followed by autoradiography and Western blotting. As the result shows, imported Ina22 was co-imunoprecipitated only with endogenous Cbp3 protein, but not with Cbp3 ${ }^{\text {ProtA }}$ (Fig. 2.32. lanes 9 and 10). The latter proves that the Cbp3-Ina22 interaction does not happen due to protein aggregation in post lysis mitochondria and therefore confirms that the newly identified INAC* ${ }^{*} \mathrm{BP}$ complex is not an artifact. 


\section{Discussion}

The mitochondrial $\mathrm{F}_{1} \mathrm{~F}_{\mathrm{o}}$-ATP synthase is the terminal enzyme of OXPHOS system that uses the proton gradient across the inner mitochondrial membrane to drive ATP synthesis. Structurally, this enzyme can be divided into two parts the hydrophilic $\mathrm{F}_{1}$, which produces ATP, and the hydrophobic $\mathrm{F}_{\mathrm{o}}$, which forms a membrane proton channel and transmits the energy from proton movement to the catalytic centers. The proton channel is formed by mitochondrial-encoded Atp6 and Atp9 proteins and, on its own, is not involved in ATP synthesis. Nuclearencoded subunits are required to couple proton movement across the membrane to ATP production. Without this coupling, the proton gradient across the inner mitochondrial membrane would dissipate without productive ATP synthesis and, therefore, only heat would be generated. Some hibernating animals, as well as children, actually use an uncoupled mitochondrial respiration in brown fat tissue for this purpose (Nicholls et al., 1978; Kozak et al., 1988; Kalinovich et al., 2017). However, in the majority of eukaryotic cells, uncoupled respiration has deleterious consequences.

It is not surprising that expression and assembly of nuclear- and mitochondrialencoded $\mathrm{F}_{1} \mathrm{~F}_{\mathrm{o}}$-ATP synthase subunits should be coordinated in order to avoid the overproduction of individual $F_{1}$ and $F_{o}$ modules. When present in excess in mitochondria, the $\mathrm{F}_{1}$ and $\mathrm{F}_{\mathrm{o}}$ modules will hydrolyze ATP and dissipate the membrane potential, respectively. Moreover, the biogenesis of the Atp9/Atp6 channel requires special regulation, as its formation prior to association with nuclear-encoded subunits is highly unfavorable. According to the recently proposed model of $\mathrm{F}_{1} \mathrm{~F}_{\mathrm{o}}$-ATP synthase assembly, Atp9 associates with Atp6 in a final assembly step, when nuclearencoded subunits are already positioned (Rak et al., 2011). However, the mechanism by which the last step is controlled remains unidentified.

The recent identification of the INA complex partially shed some light on how $\mathrm{F}_{1}$ might be connected to $\mathrm{F}_{\mathrm{o}}$. In this study, we extend upon previous knowledge and show that not only is the INA complex required for peripheral stalk and $\mathrm{F}_{1}$ biogenesis, but it is also involved in the formation of the Atp6/Atp9 proton channel, the final and most important step of $\mathrm{F}_{1} \mathrm{~F}_{\mathrm{o}}$-ATP synthase assembly. 


\subsection{Altered expression of mitochondrial-encoded $F_{1} F_{0}$-ATP synthase components in ina mutants}

In this study, we show that deletion of the INA complex alters expression of the mitochondrial-encoded $\mathrm{F}_{1} \mathrm{~F}_{\mathrm{o}}$-ATP synthase subunits Atp6, Atp8 and Atp9. Importantly, neither expression nor stability of the other mitochondrial-encoded subunits of the respiratory chain was affected, implying that the observed effect is $\mathrm{F}_{1} \mathrm{~F}_{\mathrm{o}}$-ATP synthase-specific. In vivo pulse-labeling with $\left[{ }^{35} \mathrm{~S}\right]$ methionine showed that the levels of Atp6 and Atp8 increase almost two-fold upon INA22 or INA17 deletion. As stability of the newly-translated proteins increased only slightly compared to wild type, we concluded that their production was strongly enhanced and therefore resulted in greater protein abundance. Considering that Atp6 and Atp8 are encoded on the same bi-cistronic mRNA and are present in a one to one stochiometry within the mature complex $\mathrm{V}$, their similar expression levels in various mutants is not surprising and was reported before (Rak and Tzagoloff, 2009; Rak et al., 2016).

Analyses of Atp9 levels in ina mutants gave contradicting results. In vivo pulse-labeling experiments did not reveal significant differences between the mutants and the wild type. However, when Atp9 was analyzed after in organello pulselabeling with $\left[{ }^{35} \mathrm{~S}\right]$ methionine, we clearly saw a strong reduction in Atp9 levels, of both its monomeric and its oligomeric form. The reason for these results remains unclear. We can speculate that the Atp9 protein is highly unstable and, therefore, its oligomerization and efficient assembly into complex $\mathrm{V}$ is required to reduce its turnover. It is not unlikely that the half-life of Atp25 or of the Aep1/Aep2 proteins, required for Atp9 expression and stability (Zeng et al., 2008; Finnegan et al., 1995; Payne et al., 1993; Ziaja et al., 1993), might be very short and so their constant import into mitochondria is required for efficient Atp9 biogenesis. While the required factors are constantly supplied by the cytoplasmic translation system in vivo, their translation and import into purified mitochondria in vitro is of course not possible. Thus, while the Atp9 ring is efficiently assembled into $\mathrm{F}_{1} \mathrm{~F}_{\mathrm{o}}$-ATP synthase and therefore stabilized in wild type mitochondria, its impaired assembly in ina mutants, in combination with reduced amounts of stabilizing factors in purified mitochondria, might contribute to the observed two-fold decrease in Atp9 levels. However, we do not exclude that some yet unidentified Atp9 biogenesis factors contribute to the observed phenotype in ina mutants.

Neither our results, nor a previously performed extensive analysis of mitochondrial ribosome-associated proteins, showed INAC association with the mitochondrial translation system (Kehrein et al., 2015). Therefore, we exclude INAC association with mitochondrial ribosomes and its direct regulation of Atp6/Atp8 translation. However, based on previously published data, it is known that Atp6/Atp8 expression depends to a large extent on nuclear-encoded $\mathrm{F}_{1} \mathrm{~F}_{\mathrm{o}}$-ATP synthase subunits 
(Goyon et al., 2008; Rak and Tzagoloff, 2009; Rak et al., 2016). The mechanism of Atp6/Atp8 translational regulation by a preassembled $F_{1}$ has been described (Rak and Tzagoloff, 2009; Rak et al., 2016). A preassembled $F_{1}$ is required to inhibit Smt1, a suppressor of Atp6/Atp8 translation, and therefore to stimulate Atp22 interaction with the ATP6/ATP8 mRNA (Rak et al., 2016). Taking significantly increased levels of free $\mathrm{F}_{1}$ in ina mutants into consideration, it can be speculated that these higher $F_{1}$ levels contribute to a stronger translational stimulation. However, this hypothesis requires further verification, as a direct dependence between the levels of free $\mathrm{F}_{1}$ and Atp6/Atp8 translation rate was not previously shown.

Moreover, we can not exclude that ATP6/ATP8 mRNA levels are elevated in ina mutants which results in higher translation rates. Though no evidence for a potential role of INAC in ATP6/ATP 8 transcription or mRNA maturation has been obtained so far.

To conclude, deletion of the INA complex components indirectly affects translation of Atp6, Atp8 and Atp9. Further analysis is required to shed a light on the mechanistic details of the observed phenotype.

\subsection{Atp6 processing defect as a marker for impaired $\mathrm{F}_{\mathrm{o}}$ bio- genesis}

In the yeast $S$. cerevisiae, Atp6 is synthesized with a 10 amino-acid N-terminal extension, which is cleaved by Atp23, a protease of the inter membrane space (Michon et al., 1988; Osman et al., 2007; Zeng et al., 2007c). Our analyses showed that although no preAtp6 could be detected in wild type cells, a significant amount of preAtp6 accumulates in the ina22 $\Delta$ mutant, implying that early biogenesis of Atp6 is affected. To our surprise, no Atp6 processing defect was observed in ina17 4 , though Ina17 and Ina22 form a stable complex and perform similar functions. It was previously shown that Ina22 recruits Ina17 to the $\mathrm{F}_{1} \mathrm{~F}_{\mathrm{o}}$-ATP synthase and that Ina17 loses its interaction with complex $\mathrm{V}$ components upon INA22 deletion (Lytovchenko et al., 2014). In this study, we show that Ina22 interacts with the $\mathrm{F}_{1} \mathrm{~F}_{\mathrm{o}}$-ATP synthase even in the absence of Ina17, although Ina22 amounts are drastically reduced in ina17 $\Delta$. Therefore, this observation explains why the processing of Atp6 was affected only in ina22 $\Delta$ - most likely, upon INA17 deletion, the remaining Ina22 is still functional in complex $\mathrm{V}$ biogenesis. In agreement with this, it has always been observed that INA17 deletion results in a milder phenotype compared to ina22 $\Delta$ - the mutant strain grows relatively better on non-fermentable carbon sources, less unassembled $F_{1}$ and more mature complex $\mathrm{V}$ is present in the cell (Lytovchenko et al., 2014). Therefore, we conclude that Ina22 has a more specific role for $\mathrm{F}_{1} \mathrm{~F}_{\mathrm{o}}$-ATP synthase biogenesis, whereas Ina17 is required mostly for Ina22 stabilization. It is, however, not known whether a pool of Ina17, not-associated with 
Ina22, is present in the cell and whether it has some other functions apart form Ina22 stabilization.

To understand why Atp6 processing is affected in ina22 $\Delta$, we performed an extensive analysis of Atp23, the protease responsible for Atp6 processing, using ina22 $\Delta$. To our surprise, steady-state amounts of Atp23 were not affected upon INA22 deletion, implying that Atp23 deficiency can not explain preAtp6 accumulation. To exclude the possibility that Ina22 might be involved in Atp23 recruitment to its substrate, we analyzed Atp23 association with Atp6 in ina22. Co-imunoprecipitation analysis revealed that the Atp23-Atp6 association is not impaired in ina22 $\Delta$. Therefore, Ina22 does not directly affect Atp23 function in Atp6 processing.

The observation that Atp23 overexpression completely rescues Atp6 processing in ina22 $\Delta$ led us to assume that the processing defect might be caused by increased Atp6 expression levels. Therefore, Atp23 levels in the cell might be limited and upon increased Atp6 production, the protease can not cope with an excess of its substrate. A similar processing phenotype was previously observed upon SMT1 deletion (Rak et al., 2016). Smt1 is a repressor that coordinates Atp6 and Atp8 expression according to the amount of available nuclear-encoded $\mathrm{F}_{1}$ module. Upon SMT1 deletion, Atp6/Atp8 translation increases and a significant amount of preAtp6 remains unprocessed. The Atp6 processing defect in smt1 $\Delta$ can also be rescued by Atp23 overexpression (Rak et al., 2016). This indicates that Atp23 levels are indeed rate limiting. Therefore, problems with Atp6 processing in ina22 $\Delta$ are an indirect effect of imbalanced Atp6 and Atp23 amounts.

Atp23 has a dual function in Atp6 processing and assembly (Osman et al., 2007; Zeng et al., 2007c). The processing is not essential, as yeast expressing catalyticallyinactive Atp23 are not affected in $\mathrm{F}_{1} \mathrm{~F}_{\mathrm{o}}$-ATP synthase biogenesis (Osman et al., 2007). Deletion of ATP23, however, results in the absence of mature complex V. We saw that overexpression of Atp23 in ina22 $\Delta$ partially rescues $\mathrm{F}_{1} \mathrm{~F}_{\mathrm{o}}$-ATP synthase assembly. First of all, the BN-PAGE analysis revealed that Atp23 overexpression restores complex $\mathrm{V}$ levels in ina22 $\Delta$ and less unassembled $\mathrm{F}_{1}$ is present in the mitochondria. Accordingly, complex $\mathrm{V}$ partially regains its oligomycin sensitivity due to these lower levels of free $\mathrm{F}_{1}$. Interestingly, the translation rate of Atp6 decreases in ina22 $\Delta$ upon Atp23 overexpression, which might support our initial assumption about unassembled $\mathrm{F}_{1}$ in ina22 $\Delta$ causing Atp6 translational upregulation. However, Atp23 overexpression alone is not enough to completely restore $\mathrm{F}_{1} \mathrm{~F}_{\mathrm{o}}$-ATP synthase assembly in ina22 $\Delta$ to wild type levels. Our analysis of Atp4 incorporation into complex $\mathrm{V}$ clearly showed that $\mathrm{F}_{1} \mathrm{~F}_{\mathrm{o}}$-ATP synthase assembly is still severely impaired in ina22 $\Delta$ Atp23 $\uparrow$, though it proceeds at a higher rate compared to ina22 $\Delta$. When catalytically-inactive Atp23 was overexpressed, $\mathrm{F}_{1} \mathrm{~F}_{\mathrm{o}}$-ATP synthase assembly was rescued to a lesser extent compared to the overexpression of wild type Atp23. How- 
ever, we observed that the Atp23 catalytic mutant is unstable and due to this can not be overexpressed to similar levels. Therefore, we could not conclude whether the protease activity of Atp23 is important for complex $\mathrm{V}$ assembly rescue in ina22 $\Delta$.

Two factors may contribute to the complex $\mathrm{V}$ assembly defect in ina22 $\Delta$ : (I) Defects in Atp6 biogenesis and $\mathrm{F}_{\mathrm{o}}$ assembly that can be rescued by Atp23 overexpression; (II) impaired downstream Atp9 ring assembly, that can not be restored by higher protease levels.

\subsection{INAC association with the Atp6/Atp8 biogenesis ma- chinery}

According to Rak et al., 2011, Atp6 associates with Atp8 and the peripheral stalk within a complex $\mathrm{V}$ assembly intermediate. As INAC is required for proper peripheral stalk assembly and physically associates with components of the peripheral stalk, we expected that INAC interacts at least transiently with the Atp6/Atp8 module. Until now, only Atp10 was shown to associate with Atp6/Atp8 and the peripheral stalk during their assembly, and the presumption had been made that Atp23 does not interact with the Atp6/Atp8 module (Rak et al., 2011). However, we have now shown that Atp10 stably associates with Atp23 in several complex V assembly intermediates containing newly translated Atp6 and Atp8. Therefore, our results imply that Atp23 is a component of complex $\mathrm{V}$ assembly intermediates, in contrast to what was previously published. An explanation for these controversial results might be simple. Rak et al. used a C-terminally HA-tagged Atp23 variant to analyze its interaction with the Atp6/Atp8 module. In our hands, C-terminal tagging of Atp23 affects protein stability an therefore reduces its association with the Atp6/Atp8 module. Therefore, in this study we analyzed endogenous untagged Atp23 to exclude any possible side-effects.

We initially hypothesized that the interaction between Atp10 and Atp23 is mediated by Atp6 and Atp8. However, our results showed that the association of Atp10 with Atp23 does not increase upon mitochondrial translation upregulation. This implies that either Atp10 interacts with Atp23 directly, or more likely, that all Atp23 within mitochondria is recruited to Atp6/Atp8/Atp10 complex and no free Atp23 pool exists. Therefore, Atp23 might be associated with Atp6 within two functionally-distinct complexes - one required for Atp6 processing and the other one needed for peripheral stalk assembly. We speculate that when the Atp9 ring is connected, Atp23 is released from the Atp6/Atp8/peripheral stalk module and can be recycled for Atp6 processing. Therefore, upon Atp6 translation upregulation or complex $\mathrm{V}$ assembly stall, Atp23 accumulates with an Atp6/Atp8 intermediate. However, experimental evidence for Atp23 "recycling" during $\mathrm{F}_{1} \mathrm{~F}_{\mathrm{o}}$-ATP synthase assembly is missing and this possible exciting mechanism should be inves- 
tigated further. Interestingly, feedback mechanisms were shown for the biogenesis of mitochondrial-encoded Cox1 and cytochrome $b$ that link their expression to assembly (Mick et al., 2010; Gruschke et al., 2012). However, no similar mechanism was discovered so far for complex $\mathrm{V}$ assembly. Therefore, it would be interesting to verify whether any of the known $\mathrm{F}_{1} \mathrm{~F}_{\mathrm{o}}$-ATP synthase assembly factors function in a similar way, or whether some still unidentified proteins are involved.

Our data clearly showed that INAC associates with both Atp10 and Atp23 in a $400 \mathrm{kDa}$ complex that contains newly translated Atp6 and Atp8. Interestingly, INAC interaction with Atp10 and Atp23 is of a transient nature and becomes strongly enhanced upon mitochondrial translational upregulation and accumulation of complex V assembly intermediates. The latter means that, in contrast to Atp23, free Ina17 and Ina22 pools exist in mitochondria from which the proteins are actively recruited for complex $\mathrm{V}$ assembly and, most likely, Atp6 and Atp8 are required for their recruitment. However, upon ATP22 deletion and Atp6 absence, Ina22 does not completely lose its interaction with Atp23. We speculate that this interaction might be maintained in the presence of Atp8. To verify this, the Ina22-Atp23 association should be analyzed in mitochondria devoid of both Atp6 and Atp8. Unfortunately, such an experiment can not be performed until an Atp8-specific translational activator is identified. Another explanation for the remaining Ina22-Atp23 interaction in the absence of Atp6 might be that it does not require either Atp6 or Atp8. A small pool of the INA complex might always remain associated with Atp23 and, most probably, Atp10 to monitor complex V assembly state, and their interaction is only stimulated in the presence of newly-translated Atp6 and Atp8.

It is unclear why a previously performed mass spectrometry analysis of Ina $22^{\text {ProtA }}$ isolated protein complexes after SILAC did not reveal the Ina22 interaction with components of the Atp6/Atp8 assembly module (Lytovchenko et al., 2014). As Atp6 and Atp8 are highly hydrophobic membrane proteins, it is possible that too few peptides were generated for the proteins to be identified. Moreover, INAC interaction with the Atp6/Atp8 module is transient in nature, so most likely not enough proteins were co-isolated under experimental conditions for detection. Last but not least, we can not exclude that the Protein A tag at the Ina22 C-terminus is sterically inaccessible within the Atp6/Atp8/peripheral stalk module. In either case, this study confirms that endogenous non-tagged Ina22 associates with the Atp6/Atp8 intermediate during $\mathrm{F}_{1} \mathrm{~F}_{\mathrm{o}}$-ATP synthase assembly.

\section{4 $\mathrm{F}_{1} \mathrm{~F}_{\mathrm{o}}$-ATP synthase assembly is stalled before its final step in ina22 $\Delta$}

To better understand the molecular mechanisms of INAC function, we asked what happens to $\mathrm{F}_{1} \mathrm{~F}_{\mathrm{o}}$-ATP synthase assembly intermediates upon INA22 deletion. 
We have shown that the Atp6/Atp8 module additionally contains Atp23 and transiently associates with the INA complex. The observed accumulation of Atp23 with Atp10 in ina22 $\Delta$ indicates that the Atp23-Atp10 interaction happens prior to association with the INA complex. Clearly, the accumulated Atp10-Atp23 containing complex comprises of newly translated Atp6 and Atp8, and therefore it is a true assembly intermediate. It is likely that this complex accumulates in ina22 $\Delta$ for two obvious reasons. First, the translation of Atp6 and Atp8 is upregulated almost two-fold upon INA22 deletion, therefore, simply more intermediates are present in the mitochondria. Second, the absence of the INA complex creates a bottle-neck in the assembly line and therefore slows down further assembly steps.

In this study, we showed that in contrast to the previously published $\mathrm{F}_{1} \mathrm{~F}_{\mathrm{o}}$-ATP synthase assembly model (Rak et al., 2011), F 1 interaction with the Atp6/Atp8 module and the peripheral stalk precedes its association with Atp9 ring. Therefore, a novel intermediate, containing Atp6/Atp8, the peripheral stalk and $F_{1}$ is shown for the first time. According to our results, Atp10 interacts with components of both the peripheral stalk and $F_{1}$ module transiently, meaning that the above mentioned intermediate is short-lived under normal conditions and does not accumulate to detectable levels in wild type cells, which explains why it was not identified before. In contrast to the wild type situation, a significant amount of the peripheral stalk and $\mathrm{F}_{1}$ accumulates with the Atp6/Atp8/Atp10/Atp23-containing complex in ina22 , pointing to a stall in downstream complex $\mathrm{V}$ assembly. Even more surprising, we showed that the not fully assembled peripheral stalk and $F_{1}$ are positioned on the Atp6/Atp8 module, suggesting that their de novo assembly occurs here. Therefore, the biogenesis of the nuclear and mitochondrial-encoded parts of complex $\mathrm{V}$ is spatially connected, implying the existence of a totally new regulatory mechanism.

A novel order in $\mathrm{F}_{1} \mathrm{~F}_{\mathrm{o}}$-ATP synthase assembly opens up new questions that should be addressed in the future. To start with, biogenesis of the peripheral stalk and $F_{1}$ on the Atp6/Atp8 module should be addressed. First, it is not known whether assembly of the peripheral stalk de novo happens with the help of some additional factors that were overlooked before. It can not be excluded that Atp10 and Atp23 might play a role in this process. As the Atp6/Atp8 module, peripheral stalk, $F_{1}$ and potential novel assembly factors accumulate in one complex in ina22 $\Delta$, this intermediate can be isolated and analyzed further by mass spectrometry to identify the missing components of the assembly process. Interestingly, our results indicate that Atp5 is also present in this intermediate. Previously, it was shown that Atp5 is not associated with the Atp6/Atp8/peripheral stalk module and therefore it was not clear how exactly it assembles (Rak et al., 2011). Considering how important Atp5 is for linking the peripheral stalk and $F_{1}$ together, it is not surprising that it assembles on the Atp6/Atp8 module. It would be interesting to address whether Atp5 is the last component of the peripheral stalk to be assembled in the final step 
to attach $\mathrm{F}_{1}$.

A second question relating to $\mathrm{F}_{1} \mathrm{~F}_{\mathrm{o}}$-ATP synthase assembly comes from the knowledge that $\mathrm{F}_{1}$ de novo assembly requires Atp11 and Atp12, as well as chaperones (Ackerman and Tzagoloff, 1990b; Francis and Thorsness, 2011). Therefore, it was expected that Atp11 and Atp12 should associate with Atp23, Atp10 and Atp6/Atp8, in order to assemble $F_{1}$. However, it is enigmatic how this machinery is tethered to the Atp6/Atp8 module and whether some scaffold-forming proteins are involved. ina22 $\Delta$ mitochondria might serve as a powerful tool for detailed characterization of this assembly step.

Another open question is how exactly $A T P 6 / A T P 8$ mRNA translation is regulated by the preassembled $F_{1}$ module. Moreover, it is not known if translational control is happening by a specialized protein complex or, alternatively, one of the complex $\mathrm{V}$ assembly intermediates. If Atp6/Atp8 translational control is indeed spatially separated from the assembly, where does $\mathrm{F}_{1}$ come from? The population of $\mathrm{F}_{1}$ that assembles on the Atp6/Atp8 module is, most likely, used in the assembly. Is this assembly hotspot the only place where $\mathrm{F}_{1}$ is made in the cell (and thus supplied for complex $\mathrm{V}$ biogenesis and regulation of ATP6/ATP8 mRNA synthesis), or does another one exit? The $F_{1}$ module that is involved in translational regulation might be assembled independently in another place in the mitochondrion. If the latter is the case, it would be exciting to investigate whether an interplay between these two potential $F_{1}$ pools exists during complex $\mathrm{V}$ biogenesis and whether they differ in their composition or associate with specialized proteins.

We showed that $\mathrm{F}_{1}$ dissociates from the Atp6/Atp8/Atp10/Atp23/F $/$ peripheral stalk complex under BN-PAGE conditions. Therefore, the complex is unstable and hard to detect by common biochemical approaches. This, and its low levels in wild type mitochondria, might explain why it has not been identified before.

The above discussed data confirm that in the absence of Ina22, assembly of the $\mathrm{F}_{1} \mathrm{~F}_{\mathrm{o}}$-ATP synthase is stalled and that Atp6/Atp8, together with their assembly factors Atp23 and Atp10, the peripheral stalk and $F_{1}$ module, accumulate in one complex. The only part which is missing in this assembly intermediate to complete the mature complex $\mathrm{V}$ is an Atp9 ring. Our experimental results clearly state that neither Atp10, nor Atp23, physically interact with the Atp9 ring and therefore these two proteins can not mediate the last assembly step, namely Atp9 ring connection.

\subsection{INAC mediates formation of the membrane proton chan- nel in the last step of complex $\mathrm{V}$ formation}

Immunoprecipitation of endogenous Ina22 after radiolabeling of mitochondrialencoded proteins revealed that it associates with newly translated Atp6, Atp8 and with the Atp9 ring. As INAC is not a part of the mature $\mathrm{F}_{1} \mathrm{~F}_{\mathrm{o}}$-ATP synthase, 
it was obvious that this interaction occurs within assembly intermediates. Our further analysis confirmed that Ina22 is present in two different complexes. One of them contains newly translated Atp6, Atp8 and Atp23. Another one contains the assembled Atp9 ring. It is likely that the Atp6/Atp8-containing protein complex also contains Atp10, however, due to the lack of antibodies raised against endogenous Atp10, we could not show this experimentally. Moreover, we need to analyze whether INAC associates with the Atp6/Atp8 module prior to the peripheral stalk and $\mathrm{F}_{1}$, to facilitate their incorporation into complex V. Most likely this is the case, as it was already shown that INAC facilitates peripheral stalk assembly (Lytovchenko et al., 2014). However, due to the instability of the intermediate, this question will be very difficult to address experimentally.

Our finding that Ina22 can be cross-linked to the Atp9 ring, and thus directly interacts with it, but can also be cross linked to Atp4, ultimately proves that it is present in the two final complex $\mathrm{V}$ assembly intermediates. Until now, no proteins have been discovered that might link the Atp9 ring to the Atp6/Atp8 module and, therefore, complete the $\mathrm{F}_{1} \mathrm{~F}_{\mathrm{o}}$-ATP synthase. It is worth emphasizing again how important the last step of complex $\mathrm{V}$ assembly is. Until this point, the proton channel-forming subunits, Atp6 and Atp9, are kept spatially separated in the inner mitochondrial membrane in order to avoid their premature association and proton channel formation. In was shown in bacteria that the $\mathrm{F}_{\mathrm{o}}$ module can dissipate membrane potential (Franklin et al., 2004), therefore, a specialized mechanism should exist to prevent this. We can speculate that the INA complex mediates the final complex $\mathrm{V}$ assembly step. INAC is physically present on the Atp6/Atp8 module and therefore directly monitors its assembly state. Furthermore, it is in direct contact with the Atp9 ring to prevent the proton channel from being formed before the peripheral stalk and $\mathrm{F}_{1}$ are assembled.

Two different models of INAC function can be proposed. According to the first model, INAC remains associated with Atp6/Atp8 until the peripheral stalk and $\mathrm{F}_{1}$ are correctly positioned. The correct attachment of nuclear-encoded subunits might induce conformational changes in the Atp6/Atp8 module that releases Ina22 and enables Atp9 ring attachment. On the other hand, Ina22 associated with the Atp9 ring is also released once the ring is incorporated into complex V. Whether Ina22 is recycled between Atp6/Atp8 and Atp9 intermediates during assembly is an interesting question that should be addressed. Our second hypothesis is that the connection of the two assembly intermediates might be driven by INAC dimerization, meaning that the dimer is formed by Ina proteins associated with the Atp9 ring and the Atp6/Atp8 module. Once the peripheral stalk and $F_{1}$ are positioned, slight conformational changes in the Atp6/Atp8 intermediate are induced which could drive INAC dimerization and complex V assembly. Afterwards, the dimer is released from complex V. Additionally, this hypothesis requires some factors to disassemble 
the INAC dimer. This process would be energy-dependent and therefore, it is highly unlikely that it can happen spontaneously.

Whereas Ina22 is highly unstable upon INA17 deletion, it is evident that Ina17 does not require Ina22 for its stability. Therefore, we can not exclude that Ina17 might be present in the cell in some Ina22-independent pool. However, our results indicate that Ina17 can not associate with complex $\mathrm{V}$ in the absence of Ina22. Therefore, we exclude that complex $\mathrm{V}$ assembly might be driven by Ina17-Ina22 association/dissociation. However, we can not exclude that some additional, not yet identified, proteins might play a role in the assembly and an interplay between them and INAC is possible.

It is tempting to speculate that Ina22 functions as a chaperone that interacts with proton-channel forming residues on both Atp6 and Atp9 to protect them from the membrane lipid environment. If this is true, Ina22 should be present in 1:1 stochiometry on the Atp9 ring, whereas only one Ina22 molecule would be required to shield Atp6. This is hard to address experimentally and, most probably, structural studies of purified intermediates would be required to prove whether this is the case.

\subsection{A novel model of $F_{1} F_{o}$-ATP synthase assembly}

Based on the results obtained in this study, we propose a novel model of $\mathrm{F}_{1} \mathrm{~F}_{\mathrm{o}^{-}}$ ATP synthase assembly in S. cerevisiae. Accordingly, Atp6 is synthesized with an N-terminal extension, which is cleaved by Atp23. We do not know at which assembly step exactly the cleavage occurs. It is hard to address experimentally if Atp6 association with Atp8 is required for Atp23 recruitment and, therefore, N-terminal processing. Moreover, we can only speculate that Atp6 is stabilized by Atp10 prior to processing. Most likely, Atp10 engages first with preAtp6 and Atp23 is recruited later, followed by Atp8 association. Nonetheless, experimental evidence for this is still missing. Afterwards, Atp6 associates with Atp8 within an Atp10- and Atp23containing protein complex. This complex represents an assembly-competent pool of Atp6 and Atp8 in the cell. Most importantly, the Atp6/Atp8 module functions as a platform for the assembly of the peripheral stalk and $F_{1}$. Whether some additional factors associate with this complex to facilitate assembly of the nuclear-encoded proteins is a matter of debate, but we have now shown that the INA complex interacts with this assembly intermediate. Simultaneously, the INA complex associates with a preformed Atp9 ring. In the last assembly step, INAC links the two assembly intermediates together and the mature complex $\mathrm{V}$ is produced. After the Atp9 ring is connected, INAC, Atp10 and Atp23 are released from the assembly intermediates and no longer remain associated with complex V (see Fig.3.1).

The model of $\mathrm{F}_{1} \mathrm{~F}_{\mathrm{o}}$-ATP synthase assembly proposed in this study differs by one important aspect from the previously published one (Rak et al., 2011). Though 


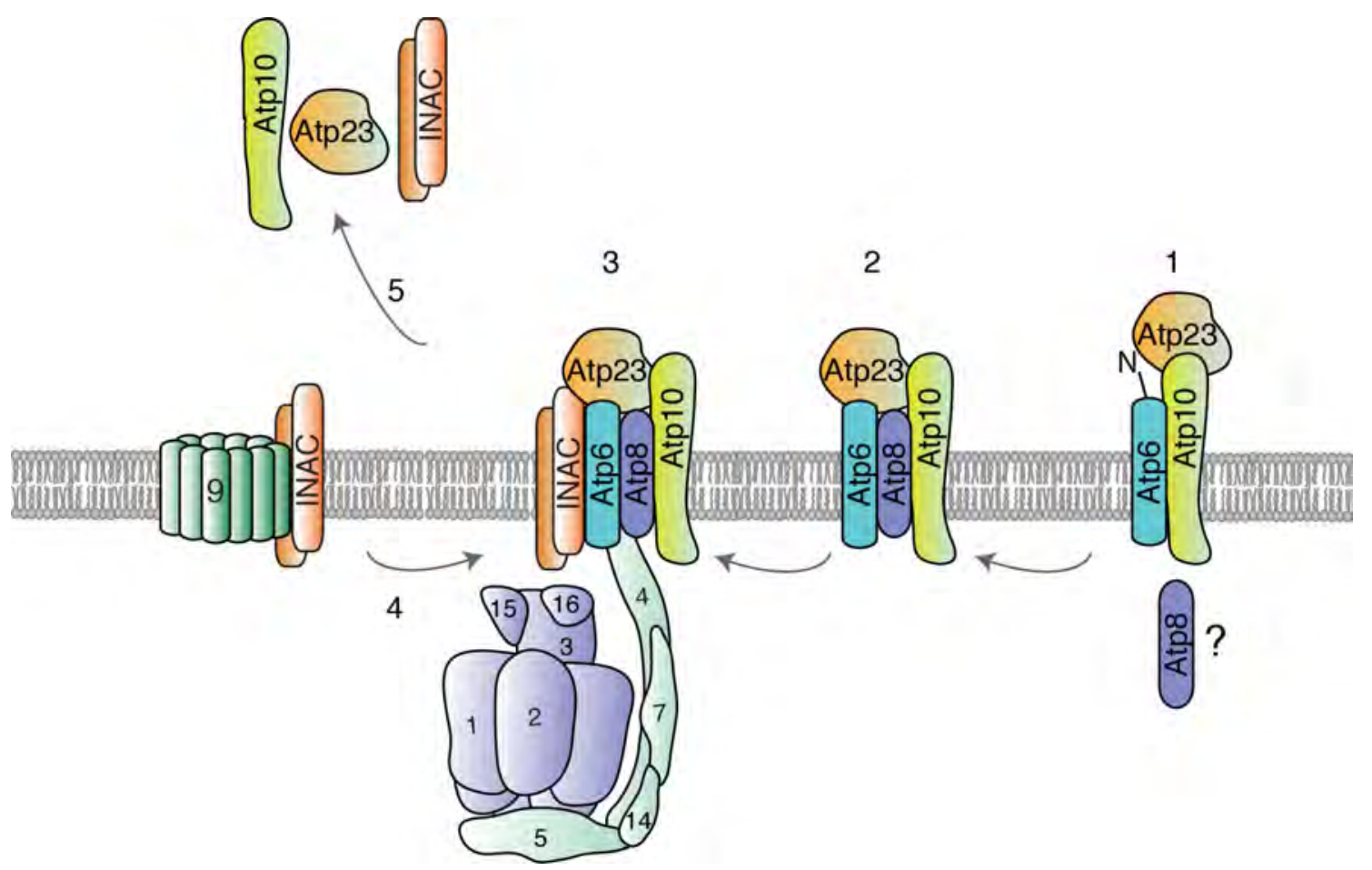

Figure 3.1 A novel model for $\mathbf{F}_{\mathbf{1}} \mathbf{F}_{\mathbf{o}}$-ATP synthase assembly. 1. After its synthesis, Atp6 engages with Atp10 and Atp23 and it N-terminus is processed by Atp23. 2. Atp6 interacts with Atp8 and remains associated with Atp10 and Atp23 within an assemblycompetent intermediate, called the Atp6/Atp8 module. 3. The peripheral stalk and $\mathrm{F}_{1}$ assemble on the Atp6/Atp8 module to generate a first major assembly intermediate. The INA complex is recruited to this intermediate to prevent an untimely Atp6/Atp9 interaction. 4. the Atp9 ring, associated with INAC, is connected to the first assembly intermediate. 5. The final assembly step triggers release of the assembly factors.

the authors identified the Atp6/Atp8 module as an autonomous assembly intermediate that is formed independently from the Atp9 ring, they claim that $\mathrm{F}_{1}$ does not associate with the Atp6/Atp8 module during complex $\mathrm{V}$ assembly. Instead, an Atp9 ring $/ F_{1}$ intermediate is formed, which associates later with the Atp6/Atp8/peripheral stalk. According to our results, an Atp6/Atp8/F $/$ peripheral stalk intermediate can be detected after IgG affinity chromatography or immunoprecipitation from mitochondria, solubilized in $1 \%$ digitonin and $160 \mathrm{mM} \mathrm{NaCl}$ to a final protein concentration of 1-2 $\mathrm{mg} / \mathrm{mL}$. Moreover, this complex is highly unstable and therefore falls apart to a single $\mathrm{F}_{1}$ unit and Atp6/Atp8/peripheral stalk module under BN-PAGE conditions. In contrast to our experimental conditions, Rak et al. used mitochondria, solubilized in $3 \%$ digitonin and $400 \mathrm{mM} \mathrm{NaCl}$ to a final concentration of $10 \mathrm{mg} / \mathrm{mL}$ to isolate complex $\mathrm{V}$ assembly intermediates and analyzed them by sucrose gradient centrifugation. We conclude that the conditions used by Rak et al. were not optimal for keeping the above mentioned intermediate stable. In agreement with this, in our hands Atp5 was co-isolated with the above mentioned intermediate, whereas Rak et al. could not co-isolate Atp5 with the Atp6/Atp8 module. When the authors tried to deplete the newly formed Atp6/Atp8 intermediate 
with anti-Atp2 antibodies, lysed mitochondria were incubated with the serum for 2 hours at room temperature, conditions that would definitely lead to $\mathrm{F}_{1}$ dissociation.

Nevertheless, despite the differences between the two models regarding this one step of complex $\mathrm{V}$ assembly, the models agree on one very important aspect association of the Atp9 ring with Atp6 and, therefore, the formation of the membrane proton channel is the final step of $\mathrm{F}_{1} \mathrm{~F}_{\mathrm{o}}$-ATP synthase biogenesis.

\subsection{Evolutionary conservation of the INA complex and the $\mathrm{F}_{1} \mathrm{~F}_{\mathrm{o}}$-ATP synthase assembly pathway}

Close homologs of Ina proteins are present only in the fungal kingdom and can not be identified in higher eukaryotes. Interestingly, our preliminary analysis showed that there is a likely correlation between the existence of an Ina22 homolog in a fungal species and the Atp9 protein being encoded in the mitochondrial genome. For example, in Neurospora crassa, the Atp9 subunit is encoded in the nuclear genome and an Ina22 sequence homolog is not found. On the other hand, in all species that possess an Ina22 homolog and whose mitochondrial genome is sequenced, Atp9 was found to be mitochondrial-encoded. However, not enough fungal mitochondrial genomes have been sequenced to perform a more comprehensive analysis and to obtain a clear conclusion. Therefore, it will be exciting to check in the future whether this correlation between Atp9 being mitochondrial-encoded and the existence of INAC homologs can be substantiated. If true, this would additionally support our hypothesis that the INA complex might be required to prevent a premature Atp6/Atp9 ring association.

Our search did not reveal obvious Ina17 or Ina22 homologs in higher eukaryotes, including humans. It is worth mentioning that the assembly of human $\mathrm{F}_{1} \mathrm{~F}_{\mathrm{o}}$-ATP synthase is less studied compared to yeast complex V assembly. Some preliminary data indicate that the assembly pathway involves formation of the $\mathrm{F}_{1} / \mathrm{Atp} 9$ ring and the Atp6/Atp8/peripheral stalk intermediates, however, clear experimental evidence for this is missing and the conclusion was made based on the model of yeast complex V assembly according to Rak et al., 2011 (Fujikawa et al., 2015).

Clearly, it can not be excluded that assembly of the human $\mathrm{F}_{1} \mathrm{~F}_{\mathrm{o}}$-ATP synthase indeed differs to some extent from yeast. The major differences between the two might be caused by the c-ring (Atp9 ring), which in humans consists of three nuclearencoded proteins (ATP5G1/2/3) and therefore might involve a different biogenesis mechanism (Anderson et al., 1981). Of all assembly factors, only the homologs for Atp23, Atp11 and Atp12 were identified in human (Wang et al., 2001; Fischer et al., 2013). Whereas the Atp11 and Atp12 (ATPAF1 and ATPAF2) function in $\mathrm{F}_{1} \mathrm{~F}_{\mathrm{o}}$-ATP synthase assembly was shown, ATP23 involvement in the biogenesis of 
complex V was not investigated. Interestingly, although human ATP23 contains a highly-conserved catalytic site required for the proteolytic activity, it is not known whether human ATP6 is expressed with a leader peptide and requires processing by ATP23.

No proteins involved in the regulation of ATP6 and ATP8 translation in human have yet been identified and therefore it is not known if translation of these mitochondrial-encoded proteins is coordinated with complex $\mathrm{V}$ assembly. Nevertheless, we anticipate that formation of the Atp6/Atp9 proton channel in human $\mathrm{F}_{1} \mathrm{~F}_{\mathrm{o}}$-ATP synthase should be regulated in order to avoid membrane potential dissipation and, most likely, a functional homolog of the INA complex might be present in human mitochondria.

Recently, the TMEM70 protein was characterized as an essential protein involved in human $\mathrm{F}_{1} \mathrm{~F}_{\mathrm{o}}$-ATP synthase assembly. Patients, harboring heterozygous mutations in TMEM70 suffer from severe complex V deficiency, leading to ultrastructural mitochondrial degeneration, encephalocardiomyopathy and neonatal pulmonary hypertension (Braczynski et al., 2015; Diodato et al., 2015; Finsterer and Kothari, 2014). A homozygous knockout of Tmem 70 in mice is embryonically lethal, pointing to the importance of the protein in complex $\mathrm{V}$ biogenesis. Interestingly, when respiratory chain complexes from Tmem $70^{-} /$mice embryos were analyzed, a severe reduction in the amount of mature complex $\mathrm{V}$ and an increase in unassembled $\mathrm{F}_{1}$ were detected, similar to the ina mutants phenotype in yeast (Vrbacky et al., 2016). The similarity of the complex $\mathrm{V}$ defects upon Tmem70 and INA complex deletions might point to a similar role of TMEM70 in human $\mathrm{F}_{\mathrm{o}}$ biogenesis and must be investigated in more detail.

So far our knowledge on human $\mathrm{F}_{1} \mathrm{~F}_{\mathrm{o}}$-ATP synthase assembly is far from complete. We do not know anything about how translation of mitochondrial-encoded ATP6 and ATP8 is regulated or how individual $F_{0}$ is assembled and linked to $F_{1}$. Current knowledge is scarce and came mainly from studies of patients in which structural subunits or assembly factors of complex $\mathrm{V}$ are mutated. However, mutations in proteins essential for complex $\mathrm{V}$ assembly might be lethal and therefore patients are rare. Since oxidative phosphorylation is not an essential process in yeast, genomewide knockout screens can be performed to identify proteins involved in OXPHOS machinery biogenesis. In some cases, the identified proteins have human homologs and so knowledge on yeast OXPHOS system assembly can be transferred to the human system. Unfortunately, this is not the case with the assembly of the human $\mathrm{F}_{1} \mathrm{~F}_{\mathrm{o}}$-ATP synthase. Therefore, another strategy must be employed and genetic screens in human cells should be performed. Screening available knockout genomic libraries, or libraries in which genes are systematically down- or upregulated, might be very useful (Gilbert et al., 2014; Arroyo et al., 2016). 


\subsection{C-terminal processing of Ina22}

The mitochondrial proteome is very dynamic and $5-10 \%$ of mitochondrial proteins are degraded within the organelle per generation under normal conditions (Augustin et al., 2005). This is achieved by mitochondrial proteases of the IMS, the matrix and the inner mitochondrial membrane. Due to such turnover, the misfolded and damaged proteome is constantly eliminated and the organelle is maintained in a healthy condition. Therefore, mitochondrial protease activity can be considered to be necessary for normal mitochondrial physiology (Baker et al., 2011).

In our study, we discovered that Ina22 undergoes C-terminal processing. Due to the lack of antibodies against the endogenous protein, C-terminally tagged Ina22 was used in the previous investigation and the processing was therefore overlooked (Lytovchenko et al., 2014). Interestingly, the protein is processed twice, resulting in the generation of two fragments of approximately 186 and 166 amino acids in length, which can be detected along with full-length Ina22. The processed C-terminal peptides are rather short in length (50 and 30 amino acids), soluble and can not be easily detected with antibodies due to their small size. Screening several known yeast IMS and IMM protease knockout mutants yielded no apparent candidate. The amino acid motifs where the processing occurs, EKVYLS and GIVTDL for the 186 and 168 fragments, respectively, do not represent a conserved cleavage site for any mitochondrial protease known so far.

We have noticed that Ina22 fragments are hard to detect by immunoblotting in intact mitochondria. Moreover, their amount, as well as the ratio between the fulllength Ina22 and the processed forms, varies greatly among different mitochondrial preparations and depends on the energy sources used (performed in collaboration with N. Vögtle from C. Meisinger group, data was not shown), implying that the processing might depend on the growth phase of the cell and physiological state of mitochondria. As the antibodies were generated against the soluble C-terminus of Ina22, we can not exclude that the recognized epitopes localize at the extreme Cterminus and, therefore, get processed. However, after immunoprecipitation of Ina22 from solubilized mitochondria, two fragments always appear significantly enriched compared to the full-length Ina22. Hence, we assume that stability of the Ina22 C-terminus decreases greatly upon solubilization. Overall, it seems like Ina22 is highly intrinsically unstable and requires additional proteins, like Ina17, for its stability. Most likely, the multiple interactions, which Ina22 maintains within protein complexes, decrease it turnover. Therefore, after solubilization, the potential stabilizing proteins dissociate from Ina22, leaving its C-terminus accessible for processing. We showed that the processing of Ina22 does not alter its function and, therefore, yeast respiration. We speculate that it most likely represents normal physiological turnover and is unlikely to have a regulatory role for complex $\mathrm{V}$ assembly. 
Nevertheless, identifying the protease involved, as well as its substrate spectrum, might contribute to our knowledge on mitochondrial protein homeostasis and its regulation.

\subsection{The elusive role of INAC ${ }^{*} \mathrm{CBP}$ association}

In the previous study (Lytovchenko et al., 2014), Ina22 and Ina17 interaction with Cbp proteins was identified. Here, we confirmed that endogenous, non-tagged Ina22 and Ina17 indeed associate with Cbp3, Cbp6 and Cbp4 and that this interaction is not a post-lysis artifact. Moreover, we showed that the interaction between INAC and Cbps takes place within a multiprotein complex, containing newly translated cytochrome $b$. The integrity of this complex depends on the presence of cytochrome $b$ and can not be maintained without it. Moreover, our intensive analyses confirmed that the above mentioned complex represents neither intermediate I or II of complex III assembly. This conclusion was made based on the observation that, first of all, the complex does not contain early complex III structural subunits and it can not be accumulated upon stalling of complex III assembly. Importantly, in ina mutants, neither the Cbp3-Cbp4 interaction and the integrity of intermediate I, nor the amount of intermediate I are altered, implying that the INAC-CBP complex does not represent a complex III assembly intermediate (data was not shown).

Despite our intensive analyses of ina and $c b p$ deletion mutants, we could not identify a phenotype associated with complex III and complex V respectively. Therefore, we conclude that this newly identified complex is not important for biogenesis of either complex III or $\mathrm{F}_{1} \mathrm{~F}_{\mathrm{o}}$-ATP synthase and its possible function remains unknown.

Cox1, Cox2 and Cox3 translational activators were previously shown to colocalize on the inner mitochondrial membrane for localized translation of their respective proteins and hence for a more efficient assembly of complex IV (Naithani et al., 2003). Moreover, yeast proteins involved in the translation of seven mitochondrial mRNAs have been shown to associate in a $900-\mathrm{kDa}$ protein complex for more efficient interaction with the transcription machinery through Nam1 (Krause et al., 2004). This was recently additionally confirmed by the identification of the mitochondrial organization of gene expression (MIOREX) complexes (Kehrein et al., 2015). Therefore, this evidence indicates that the mitochondrial gene expression system is organized into large protein complexes, which presumably facilitate gene expression in the organelle. However, neither the estimated size of the INAC/CBP complex, nor the presence of cytochrome $b$ in it allows us to assume its possible association with ribosomes and consequently a transcription/translation regulatory function. Moreover, no evidence exists so far to support a possible Ina22 function in transcription/translation control for either complex V subunits or cytochrome $b$. 
In summary, the possible role of the INAC/CBP association remains unidentified. The size of this complex (around $300 \mathrm{kDa}$ ) makes it likely that more proteins are present in it and, therefore, mass spectrometry analysis might reveal more candidates and shed a light on its function. 


\section{Conclusions and Perspectives}

Eukaryotic cells rely on efficient energy production by mitochondria. The $\mathrm{F}_{1} \mathrm{~F}_{\mathrm{o}^{-}}$ ATP synthase complex, the main energy-producing protein complex in most cells and tissues, has an intricate architecture and originates from two different genomes. Its proper biogenesis requires coordinated cytoplasmic and mitochondrial translation, as well as a highly-regulated assembly process. Failure in $\mathrm{F}_{1} \mathrm{~F}_{\mathrm{o}}$-ATP synthase biogenesis leads to decreased energy production and is deleterious for the cell. In this thesis, open questions about the biogenesis of the $\mathrm{F}_{1} \mathrm{~F}_{\mathrm{o}}$-ATP synthase were addressed. In particular, this study was devoted to the functional investigation of the INA complex, which was previously shown to participate in complex V assembly. We focused our attention on the functions of the INA complex in biogenesis of the $\mathrm{F}_{1} \mathrm{~F}_{\mathrm{o}}$-ATP synthase complex and tried to reveal its role in biogenesis of complex III. Moreover, we checked if the interplay between assemblies of both complexes exists. The main conclusions of this project can be summarized as follows:

- The INA complex consists of two mitochondrial inner membrane proteins, Ina22 and Ina17. The proteins associate with each other in a stable complex, required for maintaining Ina22 stability. Upon Ina17 deletion, Ina22 undergoes degradation and therefore its levels decrease in the cell. However, remaining Ina22 is able to associate with complex $\mathrm{V}$ assembly intermediates.

- The INA complex is not associated with the mitochondrial translation machinery and, therefore, does not directly regulate translation of Atp6, Atp8 and Atp9. However, in ina mutants, Atp6 and Atp8 translation and abundance increase almost two-fold, resulting in an accumulation of stable proteins within an assembly intermediate, the Atp6/Atp8 module. This module represents an assembly-competent pool of Atp6 and Atp8 in the mitochondria. We speculate that the observed translational upregulation is an indirect effect of elevated levels of free $\mathrm{F}_{1}$ module in ina mutants. Moreover, we assume that the Atp9 ring is destabilized in ina22 $\Delta$ and ina17 $\Delta$ due to its delayed assembly into $\mathrm{F}_{1} \mathrm{~F}_{\mathrm{o}}$-ATP synthase.

- The INA complex interacts with the Atp6/Atp8 module and its assembly factors, namely Atp23 and Atp10. In ina mutants, the Atp6/Atp8 module accumulates, implying that it associates with INAC downstream in the assembly 
pathway. Overexpression of Atp23 rescues the phenotype of the ina mutants, namely $\mathrm{F}_{1}$ dissociation, and partially restores complex $\mathrm{V}$ levels.

- In this study, we show for the first time that the peripheral stalk and $F_{1}$ assemble on the Atp6/Atp8 module, implying that an alternative assembly mechanism might exist. This includes the formation of previously unknown assembly intermediates.

- Ina22 could be cross-linked to components of the two final $\mathrm{F}_{1} \mathrm{~F}_{\mathrm{o}}$-ATP synthase assembly intermediates, the Atp6/Atp8 module and the Atp9 ring. Therefore, the INA complex is the most likely candidate to mediate the final assembly step, formation of the $\mathrm{F}_{\mathrm{o}}$ proton channel, to complete complex $\mathrm{V}$. In agreement with this, connection of the Atp6/Atp8 module to the Atp9 ring is severely delayed in ina mutants compared to wild type, resulting in less mature complex $\mathrm{V}$ being formed.

- INAC association with the cytochrome $b$ assembly machinery was investigated, but the physiological significance of this interaction was not revealed.

In this study we have shown that the INA complex is involved in the formation of the $\mathrm{F}_{\mathrm{o}}$ proton channel. However, the molecular mechanisms behind it were not investigated in detail. Therefore, it should be addressed in the future as to how exactly INAC mediates Atp9 ring attachment to Atp6 and whether it functions as a molecular chaperone for these two proteins.

Accumulation of the Atp6/Atp8 module in ina mutants might be used as a tool to isolate and further study the protein composition of this assembly intermediate. Thereby, novel $\mathrm{F}_{1} \mathrm{~F}_{\mathrm{o}}$-ATP synthase assembly factors might be identified and the exact mechanism of the peripheral stalk and $\mathrm{F}_{1}$ assembly can be determined.

In conclusion, the gained knowledge on the assembly of the yeast $\mathrm{F}_{1} \mathrm{~F}_{\mathrm{o}}$-ATP synthase has refined the current model of yeast $\mathrm{F}_{1} \mathrm{~F}_{\mathrm{o}}$-ATP synthase assembly pathway and can likely be used in the future to extend our understanding of the biogenesis of the human enzyme. 


\section{Materials and Methods}

\subsection{Materials}

\subsubsection{Chemicals}

Standard reagents used in this study were purchased from AppliChem (Darmstadt, Germany), BD (Heidelberg, Germany), Bio-Rad (Richmond, USA), BioChemica (Sauerlach, Germany), Roche (Mannheim, Germany), Merck, Novagen and Calbiochem (Darmstadt, Germany), Roth (Karlsruhe, Germany), Serva (Heidelberg, Germany), Sigma-Aldrich (Taufkirchen, Germany), Thermo Scientific (Schwerte, Germany). Enzymes and corresponding buffers were from Seikagaku Biobusiness (Tokyo, Japan), Roche (Mannheim, Germany) and Invitrogen (Darmstadt, Germany). The complete list of reagents can be found in Table 5.1.

Table 5.1 List of chemicals

\begin{tabular}{l|l}
\hline \hline & \\
Chemical & Supplier \\
\hline \hline$\left[{ }^{35}\right.$ S]-L-methionine & Hartmann Analytic \\
1-ketoglutarate & Sigma-Aldrich \\
2-mercaptoethanol & Sigma-Aldrich \\
6-aminocaproic acid & Sigma-Aldrich \\
Acetic acid & Roth \\
Acetone & Roth \\
Acrylamide/bisacrylamide (37.4:1) solution & Roth \\
Acrylamide, 4x crystallized & Roth \\
Agarose NEEO ultra-quality & Roth \\
Ammonium acetate & Roth \\
Ammonium molibdate & Merck \\
Ammonium sulfate & Roth \\
Ammonium persulfate & Roth \\
Ampicillin & AppliChem \\
Antimycine & Sigma-Aldrich \\
Ascorbic Acid & Roth \\
ATP (adenosine-5'-triphosphate) & Roche \\
Bacto ${ }^{\text {TM }}$ Agar & BD \\
Bacto ${ }^{\text {TM }}$ Peptone & BD \\
Bacto ${ }^{\text {TM }}$ Tryptone & BD \\
Bacto Yeast Extract & BD \\
Bio-Rad Protein Assay & Bio-Rad \\
& \\
\hline
\end{tabular}


Table 5.1 - Continued from previous page

\begin{tabular}{|c|c|}
\hline Chemical & Supplier \\
\hline Bis-Acrylamide & Roth \\
\hline Bis-Tris Buffer grade & AppliChem \\
\hline Bovine serum albumin & Sigma-Aldrich \\
\hline Chloramphenicol & Sigma-Aldrich \\
\hline CNBr activated sepharose 4B & GE Healthcare \\
\hline Complete EDTA-free protease inhibitor mix & Roche \\
\hline Coomassie Brilliant Blue G-250 & Serva \\
\hline Coomassie Brilliant Blue R-250 & Serva \\
\hline Copper (II) sulfate, pentahydrate & Merck \\
\hline Creatine kinase & Roche \\
\hline Creatine phosphate & Roche \\
\hline CSM-HIS & MP Biomedicals \\
\hline Cycloheximide & BioChemica \\
\hline Cytochrome c from bovine heart & Sigma-Aldrich \\
\hline DAB (diaminobenzidine) & Sigma-Aldrich \\
\hline DDM (n-Dodecyl- $\beta$-D-maltoside) & Sigma-Aldrich \\
\hline DFDNB (1,5-difluoro-2,4-dinitrobenzene) & Thermo Scientific \\
\hline Digitonin & Calbiochem \\
\hline DMP (dimethyl pimelimidate dihydrochloride) & Sigma Aldrich \\
\hline DMSO (dimethylsulfoxide) & AppliChem \\
\hline DNA ladder mix "Gene Ruler" & Fermentas \\
\hline DSP (dithiobis(succinimidyl propionate)) & Thermo Scientific \\
\hline DSS (disuccinimidyl suberate) & Thermo Scientific \\
\hline DTT (1,4-dithiothreitol) & Roth \\
\hline EDTA (ethylenediaminetetraacetic acid) & Roth \\
\hline Ethanol & Roth \\
\hline Ethidium bromide $0.07 \% \mathrm{w} / \mathrm{v}$ & AppliChem \\
\hline Geneticin 418 Sulfate & Invitrogen \\
\hline Galactose, $\mathrm{D}(+)$ & Roth \\
\hline Glucose, $\mathrm{D}(+)$ & Roth \\
\hline Glycerol & Sigma-Aldrich \\
\hline Glycine & Roth \\
\hline GTP (9-ß-D-ribofuranosylguanin-5'-triphosphat) & Sigma-Aldrich \\
\hline HEPES (4-(2-hydroxyethyl)-1-piperazineethanesulfonic acid ) & Roth \\
\hline Herring sperm DNA & Promega \\
\hline Hydrochloric acid $37 \% \mathrm{w} / \mathrm{v}$ & Roth \\
\hline IgG from human serum & Sigma Aldrich \\
\hline IgG protein standard & BioRad \\
\hline Imidazole & Sigma Aldrich \\
\hline Lead (II) nitrate & Merck \\
\hline Lithium acetate & AppliChem \\
\hline Magnesium chloride heptahydrate & Merck \\
\hline Magnesium sulfate heptahydrate & Roth \\
\hline Methanol & Roth \\
\hline Methionine & Roth \\
\hline MOPS (morpholinopropanesulfonic acid) & Sigma-Aldrich \\
\hline NADH (nicotinamide adenine dinucleotide) & Roche \\
\hline $\mathrm{Ni}^{2+}$-NTA agarose & Quiagen \\
\hline Nourseothricin & Werner BioAgents \\
\hline Nucleotides for PCR & Metabion \\
\hline Oligomycine & Sigma-Aldrich \\
\hline OrangeG & Sigma \\
\hline Orthi-phosphoric acid & Merck \\
\hline PEG-4000 (polyethylene glycol 4000) & Merck \\
\hline Phosphoenolpyruvate & Sigma-Aldrich \\
\hline PMSF (phenylmethanesulfonylfluoride) & Roth \\
\hline Potassium chloride & Roth \\
\hline Potassium dihydrogen phosphate & Merck \\
\hline
\end{tabular}

Continued on the next page 
Table 5.1 - Continued from previous page

\begin{tabular}{l|l}
\hline Chemical & Supplier \\
\hline Potassium hydroxid & Roth \\
Proteinase inhibitor coctail & Roche Applied Science \\
Proteinase K & Roche \\
SDS (sodium dodecyl sulfate) & Serva \\
SDS-PAGE protein standard & Serva \\
Sepharose 4B & GE Healthcare \\
SnakeSkin dialysis membrane (7000Da-MW cut-off) & Thermo Scientific \\
Sodium azide & Roth \\
Sodium borate & Sigma Aldrich \\
Sodium chloride & Roth \\
Sodium deoxycholate & Sigma-Aldrich \\
Sodium hydroxide & Roth \\
Sodium bicarbonate & Merck \\
Sodium hydrogencarbonate & Merck \\
Sorbitol & Roth \\
Sucrose & Roth \\
Sulfuric acid & Roth \\
TCA (trichloroacetic acid) & Merck \\
TEMED (tetramethylethylenediamine) & Roth \\
TEV protease & Invitrogen \\
Tricine & Roth \\
Tris (tris(hydroxymethyl)aminomethane) & Roth \\
Triton X-100 & Sigma-Aldrich \\
Tween-20 & Roth \\
Urea & Roth \\
Valinomycine & Sigma-Aldrich \\
Yeast nitrogen base without amino acids & BD \\
Zymolyase 20 T & Seikagaku Biobusiness \\
\hline \hline
\end{tabular}

\subsubsection{Buffers and solutions}

Recipes for the buffers used are given in Table 5.2. All buffers were prepared using Milli-Q deionized water and analytical grade chemicals. Compositions of all buffers are given in $(1 \mathrm{x})$ dilution. Media for yeast growth and transformation were autoclaved/filtered after preparation.

Table 5.2 Buffers and solutions

\begin{tabular}{l|l}
\hline \hline Buffer & Components \\
\hline \hline AVO mix & $1 \mathrm{mM}$ antimycin, 0.1 mM valinomycin, $2 \mathrm{mM}$ oligomycin in ethanol \\
Blotting buffer & $25 \mathrm{mM}$ Tris, $192 \mathrm{mM}$ glycine, $10 \%$ methanol \\
BN anode buffer & $50 \mathrm{mM}$ Bis-Tris $\mathrm{pH} 7.0$ \\
BN cathode buffer & $50 \mathrm{mM}$ tricine, $15 \mathrm{mM}$ Bis-Tris, with or without 0.2\% Coomassie \\
& Brilliant Blue G-250 \\
BN gel buffer & $67 \mathrm{mM} 6$-aminocaproic acid, $50 \mathrm{mM}$ Bis-Tris pH 7.0 \\
BN sample loading buffer & $0.5 \%$ w/v) Coomassie Brilliant Blue G-250, $50 \mathrm{mM}$ 6-aminocaproic \\
& acid, $10 \mathrm{mM}$ Bis-Tris pH 7.0 \\
& \\
\hline
\end{tabular}


Table 5.2 - Continued from previous page

\begin{tabular}{|c|c|}
\hline Buffer & Components \\
\hline $\begin{array}{l}\text { BSA (bovine serum albumin)- } \\
\text { import buffer }\end{array}$ & $\begin{array}{l}3 \%(\mathrm{w} / \mathrm{v}) \text { fatty acid free bovine serum albumin, } 250 \mathrm{mM} \text { sucrose, } 80 \\
\mathrm{mM} \mathrm{KCl}, 5 \mathrm{mM} \mathrm{MgCl} 2,10 \mathrm{mM} \text { MOPS-KOH pH 7.2, } 5 \mathrm{mM} \text { methio- } \\
\text { nine }\end{array}$ \\
\hline Carrier DNA & Herring sperm DNA $(10 \mathrm{mg} / \mathrm{ml})$ in TE buffer \\
\hline $\begin{array}{l}\text { Colloidal Coomassie staining so- } \\
\text { lution }\end{array}$ & $\begin{array}{l}0.1 \%(\mathrm{w} / \mathrm{v}) \text { Coomassie Brilliant Blue G-250, } 2 \%(\mathrm{w} / \mathrm{v}) \text { phosphoric } \\
\text { acid, } 10 \%(\mathrm{w} / \mathrm{v}) \text { ammonium sulfate, } 20 \% \text { methanol }\end{array}$ \\
\hline Coomassie staining solution & $2.5 \mathrm{~g} / \mathrm{l}$ Coomassie Brilliant Blue R-250, $40 \%$ ethanol, $10 \%$ acetic acid \\
\hline Crosslinking buffer & 20 mM HEPES pH 7.4, 100 mM NaCl \\
\hline DDM solubilization buffer & $\begin{array}{l}20 \mathrm{mM} \text { Tris/HCl, } 150 \mathrm{mM} \mathrm{NaCl}, 10 \% \text { glycerol, } 1 \mathrm{mM} \text { EDTA, } 0.6 \% \\
\text { DDM }\end{array}$ \\
\hline Destaining solution & $30 \%$ ethanol, $10 \%$ acetic acid \\
\hline $\begin{array}{l}\text { Digitonin solubilization buffer } \\
\text { (low-stringent) }\end{array}$ & $\begin{array}{l}20 \mathrm{mM} \text { Tris } / \mathrm{HCl} \mathrm{pH} 7.4,150 \mathrm{mM} \mathrm{NaCl}, 10 \% \text { glycerol, } 1 \mathrm{mM} \text { EDTA, } \\
1 \% \text { digitonin }\end{array}$ \\
\hline $\begin{array}{l}\text { Digitonin solubilization buffer } \\
\text { (hight-stringent) }\end{array}$ & $\begin{array}{l}10 \mathrm{mM} \text { Tris } / \mathrm{HCl} \mathrm{pH} 9.0,400 \mathrm{mM} \mathrm{NaCl}, 10 \% \text { glycerol, } 10 \mathrm{mM} \text { imi- } \\
\text { dazole, } 1 \mathrm{mM} \text { EDTA, } 3 \% \text { digitonin }\end{array}$ \\
\hline DNA loading dye & $10 \%$ sucrose, $0.25 \%$ OrangeG \\
\hline DTT buffer & $10 \mathrm{mM}$ DTT, $100 \mathrm{mM}$ Tris $/ \mathrm{H}_{2} \mathrm{SO}_{4} \mathrm{pH} 9.4$ \\
\hline $\mathrm{EM}$ & $10 \mathrm{mM}$ MOPS-KOH pH 7.2, 1 mM EDTA \\
\hline Fixation solution & $50 \%$ methanol, $2 \%$ phosphoric acid \\
\hline His-free media & $\begin{array}{l}6.7 \%(\mathrm{w} / \mathrm{v}) \text { yeast nitrogen base w/o amino acids, } 20 \%(\mathrm{w} / \mathrm{v}) \text { sucrose, } \\
25 \%(\mathrm{w} / \mathrm{v}) \text { agar (for plates), } 0.77 \mathrm{~g} / \mathrm{l} \text { CSM-HIS }\end{array}$ \\
\hline Homogenization buffer & $\begin{array}{l}0.6 \mathrm{M} \text { sorbitol, } 10 \mathrm{mM} \text { Tris/HCl pH } 7.4,1 \mathrm{mM} \text { EDTA, } 0.2 \%(\mathrm{w} / \mathrm{v}) \\
\text { fatty acid free BSA, } 1 \mathrm{mM} \text { PMSF }\end{array}$ \\
\hline LB medium (Lysogeny broth) & $1 \%(\mathrm{w} / \mathrm{v})$ tryptone, $0.5 \%(\mathrm{w} / \mathrm{v}) \mathrm{NaCl}, 1 \%(\mathrm{w} / \mathrm{v})$ yeast extract \\
\hline Lithium acetate/PEG & $\begin{array}{l}0.1 \mathrm{M} \text { lithium acetate, } 40 \% \text { polyethylene glycol } 4000 \text { in water, filter- } \\
\text { sterilized }\end{array}$ \\
\hline Na-deoxycholate & $1.25 \%(\mathrm{w} / \mathrm{v})$ sodium deoxycholate in water \\
\hline PMSF stock & $0.2 \mathrm{M}$ phenylmethylsulfonyl fluoride in ethanol \\
\hline $\begin{array}{l}\text { Potassium phosphate buffer } \mathrm{pH} \\
7.2\end{array}$ & $71.7 \%(\mathrm{w} / \mathrm{v}) \mathrm{K}_{2} \mathrm{HPO}_{4}, 28.3 \%(\mathrm{w} / \mathrm{v}) \mathrm{KH}_{2} \mathrm{PO}_{4}$ \\
\hline $\begin{array}{l}\text { Potassium phosphate buffer } \mathrm{pH} \\
7.4\end{array}$ & $80.2 \%(\mathrm{w} / \mathrm{v}) \mathrm{K}_{2} \mathrm{HPO}_{4}, 19.8 \%(\mathrm{w} / \mathrm{v}) \mathrm{KH}_{2} \mathrm{PO}_{4}$ \\
\hline Resolving gel (for SDS-PAGE) & $\begin{array}{l}\text { 10- } 16 \% \text { acrylamide, } 80 \mathrm{mM} \text { Tris- } \mathrm{HCl} \mathrm{pH} 6.8,0.1 \% \text { SDS, } 0.1 \% \text { ammo- } \\
\text { niumperoxodisulfate, } 0.05 \% \text { TEMED }\end{array}$ \\
\hline SDS loading buffer & $\begin{array}{l}10 \% \text { glycerol, } 2 \%(\mathrm{w} / \mathrm{v}) \text { SDS, } 0.01 \% \text { bromophenole blue, } 60 \mathrm{mM} \\
\text { Tris } / \mathrm{HCl} \mathrm{pH} 6.8\end{array}$ \\
\hline SDS running buffer & $25 \mathrm{mM}$ Tris, $192 \mathrm{mM}$ glycine, $0.1 \%$ (w/v) SDS \\
\hline SEM & $250 \mathrm{mM}$ sucrose, $10 \mathrm{mM}$ MOPS-KOH $\mathrm{pH}$ 7.2, $1 \mathrm{mM}$ EDTA \\
\hline Stacking gel (for SDS-PAGE) & $\begin{array}{l}4 \%(\mathrm{w} / \mathrm{v}) \text { arylamide, } 0.1 \%(\mathrm{w} / \mathrm{v}) \mathrm{SDS}, 380 \mathrm{mM} \text { Tris- } \mathrm{HCl} \mathrm{pH} 8.8 \text {, } \\
0.1 \%(\mathrm{w} / \mathrm{v}) \text { ammonium peroxodisulfate, } 0.05 \% \text { TEMED }\end{array}$ \\
\hline TAE buffer & $40 \mathrm{mM}$ Tris/acetate $\mathrm{pH} 8.0,2 \mathrm{mM}$ EDTA \\
\hline $\begin{array}{l}\text { TBST (Tris-Buffered Saline and } \\
\text { Tween-20) }\end{array}$ & $50 \mathrm{mM}$ Tris, $150 \mathrm{mM} \mathrm{NaCl}, 0.05 \%$ Tween-20 \\
\hline TBS (Tris-Buffered Saline) & $50 \mathrm{mM}$ Tris, $150 \mathrm{mM} \mathrm{NaCl}$ \\
\hline TCA solution & $72 \%$ trichloracetic acid in water \\
\hline TE buffer & $10 \mathrm{mM}$ Tris $\mathrm{pH}$ 8.0, $1 \mathrm{mM}$ EDTA \\
\hline Ura-free media & $\begin{array}{l}6.7 \%(\mathrm{w} / \mathrm{v}) \text { yeast nitrogen base w/o amino acids, } 20 \%(\mathrm{w} / \mathrm{v}) \text { sucrose, } \\
25 \%(\mathrm{w} / \mathrm{v}) \text { agar (for plates), } 0.77 \mathrm{~g} / \mathrm{l} \text { CSM-URA }\end{array}$ \\
\hline Urea acrylamide solution & $60 \%(\mathrm{w} / \mathrm{v})$ Acrylamide, $0.8 \%(\mathrm{w} / \mathrm{v})$ Bis-Acrylamide \\
\hline Washing buffer & $\begin{array}{l}0.3 \% \text { digitonin, } 20 \mathrm{mM} \text { Tris/ } \mathrm{HCl} \mathrm{pH} \mathrm{7.4,100} \mathrm{mM} \mathrm{NaCl,} 10 \% \text { glycerol, } \\
1 \mathrm{mM} \text { PMSF, } 1 \mathrm{mM} \text { EDTA }\end{array}$ \\
\hline YPAD $(2 \mathrm{x})$ & $\begin{array}{l}2 \%(\mathrm{w} / \mathrm{v}) \text { yeast extract, } 4 \%(\mathrm{w} / \mathrm{v}) \text { peptone, } 4 \%(\mathrm{w} / \mathrm{v}) \text { glucose }, 0.008 \% \\
(\mathrm{w} / \mathrm{v}) \text { adenine hemisulfate, autoclaved }\end{array}$ \\
\hline YPD & $1 \%$ yeast extract, $2 \%$ peptone, $2 \%$ glucose, autoclaved \\
\hline YPG & $\begin{array}{l}1 \%(\mathrm{w} / \mathrm{v}) \text { yeast extract, } 2 \%(\mathrm{w} / \mathrm{v}) \text { peptone, } 3 \%(\mathrm{w} / \mathrm{v}) \text { glycerol, auto- } \\
\text { claved }\end{array}$ \\
\hline YPGal & $\begin{array}{l}1 \%(\mathrm{w} / \mathrm{v}) \text { yeast extract, } 2 \%(\mathrm{w} / \mathrm{v}) \text { peptone, } 2 \%(\mathrm{w} / \mathrm{v}) \text { galactose, au- } \\
\text { toclaved }\end{array}$ \\
\hline
\end{tabular}

Continued on the next page 
Table 5.2-Continued from previous page

\begin{tabular}{l|l}
\hline Buffer & Components \\
\hline Zymolyase buffer & $1.2 \mathrm{M}$ sorbitol, $20 \mathrm{mM}$ potassium phosphate pH 7.4 \\
1.5xTranslation buffer & $900 \mathrm{mM}$ sorbitol, 225 mM KCl, 22.5 mM potassium phosphate buffer \\
& $\mathrm{pH} 7.2,30 \mathrm{mM}$ Tris $/ \mathrm{HCl} \mathrm{pH} 7.4,4.5 \mathrm{mg} / \mathrm{ml} \mathrm{BSA}, 6 \mathrm{mM}$ ATP, 0.75 \\
& $\mathrm{mM} \mathrm{GTP}, 9 \mathrm{mM} \alpha$-ketoglutarate, $10 \mathrm{mM}$ Creatine phosphate, 10 \\
& $\mu \mathrm{g} / \mathrm{mL}$ creatine kinase, $0.15 \mathrm{mM}$ amino acid mix (Met-free), 7.5 \\
& $\mu \mathrm{g} / \mathrm{ml}$ cycloheximide, $19 \mathrm{mM} \mathrm{MgSO}_{4}$ \\
\hline
\end{tabular}

\subsubsection{Kits and disposables}

Disposables and commercial kits used in this study together with their suppliers are listed in Table 5.3. Kits were used and stored according to the manufacturer's instructions.

Table 5.3 Kits and disposables

\begin{tabular}{l|l}
\hline \hline & \\
Product & Supplier \\
\hline \hline anti-FLAG-M2 Affinity gel & Sigma-Aldrich \\
Alkaline phosphatase, shrimp & Roche Applied Science \\
CELLSTAR ${ }^{\circledR}$ Centrifuge Tubes $15 \mathrm{ml}, 50 \mathrm{ml}$ & Geiner Bio-One \\
ECL Plus Western Blotting Detection Reagent & GE Healthcare \\
FastDigest Restriction enzymes & Fermentas/Thermo Scientific \\
FLAG-peptide & Sigma-Aldrich \\
Flexi ${ }^{\circledR}$ Rabbit Reticulocyte Lysate System & Promega \\
GeneRuler DNA Ladder Mix & Fermentas/Thermo Scientific \\
High Molecular Weight Calibration Kit & GE Healthcare \\
Hyperfilm ${ }^{\text {TM }}$ ECL films & GE Healthcare \\
Immobilon-P Transfer membrane & Millipore \\
KOD Hot Start DNA Polymerase & Novagen \\
Microtube 1.5 ml and 2.0 ml & Sarstedt \\
Minisart syringe filters & Sartorius AG \\
Pipette tips $10 \mu l, 200 \mu l$ and 1 ml & Sarstedt \\
Protein A sepharose CL 4B & GE Healthcare \\
Rapid ligation kit & Thermo Scientific \\
Roti-Quant ${ }^{\circledR}$ & Roth \\
SP6 mMESSAGE mMACHINE & Ambion ${ }^{\circledR}$, Life Technologies ${ }^{\text {TM }}$ \\
Spin Columns Mobicol "Classic" & MoBiTech \\
TNT ${ }^{\circledR}$ Quick coupled Transcription/Translation System & Promega \\
Tobacco Etch Virus (TEV) protease & Sigma-Aldrich \\
Unstained SDS-PAGE protein Marker, $6.5-200 \mathrm{kDa}$ & Serva (Heidelberg, Germany) \\
Wizard ${ }^{\circledR}$ Plus SV Gel and PCR DNA Purification System & Promega \\
Wizard ${ }^{\circledR}$ SV Minipreps & Promega \\
\hline \hline
\end{tabular}

\subsubsection{Equipment}

Laboratory equipment used in this study and suppliers are listed in Table 5.4. 
Table 5.4 Equipment

\begin{tabular}{|c|c|c|}
\hline Product & Model & Supplier \\
\hline \multirow{6}{*}{ Electrophoresis } & EPS 601 power supply & GE Healthcare \\
\hline & Hoefer SE600 Ruby Blue native system & GE Healthcare \\
\hline & Mini-PROTEAN ${ }^{\circledR} 3$ Cell & Bio-Rad \\
\hline & Mini-Sub ${ }^{\circledR}$ Cell GT Cell & Bio-Rad \\
\hline & PowerPac ${ }^{\mathrm{TM}}$ HC Power Supply & Bio-Rad \\
\hline & Semi Dry Blotting Chamber & PEQLAB Biotechnologie \\
\hline \multirow{8}{*}{ Centrifuges } & Sorvall ${ }^{\circledR}$ RC 12BP & Thermo Scientific \\
\hline & Sorvall ${ }^{\circledR}$ RC6 Plus & Thermo Scientific \\
\hline & OptimaTMDX-XP & Beckman Coulter \\
\hline & $5804 \mathrm{R}$ & Eppendorf \\
\hline & $12-\mathrm{MC}$ & Beckman Coulter \\
\hline & $5415 \mathrm{R}$ & Eppendorf \\
\hline & $5417 \mathrm{R}$ & Eppendorf \\
\hline & 5424 & Eppendorf \\
\hline \multirow{9}{*}{ Rotors } & Sorvall ${ }^{\circledR}$ F14S- $6 x 250 \mathrm{Y}$ & Thermo Scientific \\
\hline & Sorvall ${ }^{\circledR}$ F10S- 6x500Y & Thermo Sientific \\
\hline & Sorvall ${ }^{\circledR}$ SS-34 & Thermo Scientific \\
\hline & Sorvall $^{\circledR}$ H-12000 & Thermo Scientific \\
\hline & F45-24-11 & Eppendorf \\
\hline & F $45-30-11$ & Eppendorf \\
\hline & FA-45-24-11 & Eppendorf \\
\hline & TLA-55 & Beckman Coulter \\
\hline & A-4-44 & Eppendorf \\
\hline \multirow{8}{*}{ Scanners } & Agfa Curix 60 Developing machine & Agfa \\
\hline & Autoradiography Storage Phosphor Screen & GE Healthcare \\
\hline & BioPhotometer & Eppendorf \\
\hline & iMark $^{\mathrm{TM}} 1300$ Spectrophotometer & GE Healthcare \\
\hline & LAS 1000 & FujiFilm \\
\hline & Starion FLA-9000 & FujiFilm \\
\hline & Storm 820 Phosphorimager & GE Healthcare \\
\hline & UVsolo TS transilluminator & Biometra \\
\hline \multirow{20}{*}{ Other } & Autoclave Systec DX-200 & Systec \\
\hline & Balance BP $3100 \mathrm{P}$ & Sartorius \\
\hline & Branson Sonifier 450 & G. Heinemann, Schwäbisch Gmünd \\
\hline & Electronic Digital Balance Kern ABJ 220-4M & KERN Son \\
\hline & EmulsiFlex C5 & Avestin \\
\hline & Excella ${ }^{\circledR}$ E10 platform shaker & New Brunswick Scientific \\
\hline & G25 Shaker Incubator & New Brunswick Scientific \\
\hline & Innova ${ }^{\circledR} 44$ Incubator shaker & New Brunswick Scientific \\
\hline & GeneQuant $^{\mathrm{TM}} 1300$ Spectrophotometer & GE Healthcare \\
\hline & Magnetic Stirrer MR 3001 & Heidolph \\
\hline & Milli-Q-Water purification system & Millipore \\
\hline & NanoVue ${ }^{\mathrm{TM}}$ Spectrophotometer & GE Healthcare \\
\hline & pH-meter & InoLab \\
\hline & Pipettes & Gilson \\
\hline & Potter S glass-Teflon Homogenizer & Sartorius AG \\
\hline & Termomixer Comfort & Eppendorf \\
\hline & TPersonal 48 thermocycler & Biometra \\
\hline & UVsolo TS transilluminator & Biometra \\
\hline & Vacuum gel drier & Scie-Plas \\
\hline & Vortex-Genie 2 & Scientific Industries \\
\hline
\end{tabular}




\subsubsection{Software}

List of software used for data analyses, image processing and documentation of data is given in Table 5.5.

Table 5.5 Software used in this study

\begin{tabular}{l|l}
\hline \hline & \\
Software & Company \\
\hline \hline Adobe Illustrator CS6 & Adobe Systems, San Jose, CA, USA \\
Adobe Photoshop CS6 & Adobe Systems, San Jose, CA, USA \\
DataGraph 3.1.1 & Visual Data Tools, Inc. \\
Geneious Pro 5.3.6 & Biomatters, Auckland, New Zealand \\
ImageQuant TL & GE Healthcare BioSciences AB, Uppsala, Sweden \\
Microsoft Office & Microsoft Corporation, Redmond, WA, USA \\
Genious Pro 4.8.3 & Biomatters Ltd. \\
TeXShop 3.26 & Richard Koch, Mathematics Department, University of Oregon \\
\hline \hline
\end{tabular}

\subsubsection{Antibodies}

Rabbit polyclonal antisera were used for detection of Saccharomyces cerevisiae proteins. For the detection of HA-tagged and FLAG-taged yeast proteins corresponding mouse monoclonal antibodies were used (Sigma-Aldrich). Horseradish peroxidase (HRP)-conjugated goat anti-rabbit and goat anti-mouse antibodies (Jackson ImmunoResearch) were used as secondary antibodies. The complete list of antibodies is given in Table 5.6.

Table 5.6 Antibodies used in this study

\begin{tabular}{l|l}
\hline \hline & Antibody type \\
\hline \hline Intigen & Rabbit polyclonal \\
Ina17 & Rabbit polyclonal \\
Cbp3 & Rabbit polyclonal \\
Cbp4 & Rabbit polyclonal \\
Aco1 & Rabbit polyclonal \\
Atp2 & Rabbit polyclonal \\
Atp3 & Rabbit polyclonal \\
Atp14 & Rabbit polyclonal \\
Atp20 & Rabbit polyclonal \\
Atp4 & Rabbit polyclonal \\
Atp5 & Rabbit polyclonal \\
Atp23 & Rabbit polyclonal \\
Coa3 & Rabbit polyclonal \\
Cor1 & Rabbit polyclonal \\
\hline
\end{tabular}


Table 5.6 - Continued from previous page

\begin{tabular}{l|l}
\hline Antigen & Antibody type \\
\hline Cox1 & Rabbit polyclonal \\
Cox13 & Rabbit polyclonal \\
Cox14 & Rabbit poluclonal \\
Cox2 & Rabbit polyclonal \\
Cyt1 & Rabbit polyclonal \\
HA tag & Mouse Monoclonal \\
Mdm38 & Rabbit polyclonal \\
Mic10 & Rabbit polyclonal \\
Qcr8 & Rabbit polyclonal \\
Rip1 & Rabbit polyclonal \\
Tim44 & Rabbit polyclonal \\
Mrpl4 & Rabbit polyclonal \\
Mrpl36 & Rabbit polyclonal \\
Mrpl39 & Rabbit polyclonal \\
Tom70 & Rabbit polyclonal \\
Goat anti-rabbit, HRP conjugated & Goat polyclonal \\
Goat anti-mouse, HRP conjugated & Goat polyclonal \\
FLAG tag & Mouse monoclonal \\
Protein A tag, HRP-conjugated & Rabbit polyclonal \\
\hline \hline
\end{tabular}

\subsubsection{Yeast strains used in this study}

All yeast strains used in this study are derivatives of BY4741 strain (Brachmann et al., 1998), except for $\operatorname{cbp} 1 \Delta$ strain, generated from intronless XMP171. Wild type and newly generated yeast strains used in this study are listed in Table 5.7.

Table 5.7 Yeast strains

\begin{tabular}{|c|c|c|}
\hline Strain & Genotype & Reference \\
\hline$\overline{\mathrm{BY} 4741}$ & MATa; his3 $\Delta 1 ;$ leu2 $\Delta 0 ;$ met15 $\Delta 0 ;$ ura3 $\Delta 0$ & Euroscarf \\
\hline XMP171 & $\begin{array}{l}\text { MATa leu2-3, 112 ura3-52 lys2 arg8:hisG }\left[\text { cox1::ARG } 8^{m}\right. \\
\text { cox2::COX1 COX2] }\end{array}$ & $\begin{array}{l}\text { Perez- } \\
\text { Martinez } \\
\text { et al, } 2003\end{array}$ \\
\hline ina17s & 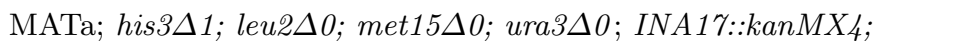 & Euroscarf \\
\hline ina22 $\Delta$ & 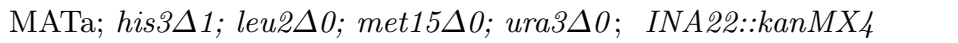 & Euroscarf \\
\hline Ina $22^{1-188}$ & 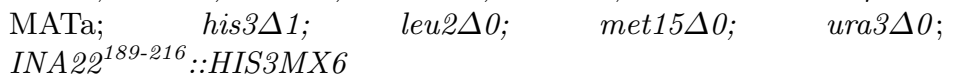 & This study \\
\hline Ina $22^{1-168}$ & $\begin{array}{lllll}\text { MATa; } & \text { his3 } \Delta 1 ; & \text { leu2 } \Delta 0 ; & \text { met } 15 \Delta 0 ; & \text { ura3 } \Delta 0 ; \\
\text { INA222 } & \text { 169-216 }: \text { HIS3MX6 } & & \end{array}$ & This study \\
\hline Atp23个 & 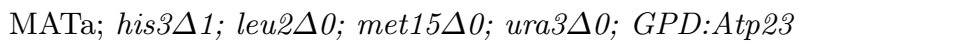 & This study \\
\hline ina22 $\Delta \mathrm{Atp} 23 \uparrow$ & $\begin{array}{l}\text { MATa; his3 } 1 \text {; leu2 } \Delta 0 ; \text { met15 } 0 \text {; ura3 } \Delta 0 ; \text { INA22::kanMX4; } \\
\text { GPD:Atp23; }\end{array}$ & This study \\
\hline ina22 $\Delta \mathrm{Atp} 23^{\mathrm{H} 128 \mathrm{~A}} \uparrow$ & $\begin{array}{l}\text { MATa; his3 } \Delta 1 ; \text { leu2 } \Delta 0 ; \text { met15 } \Delta 0 ; \text { ura3 } \Delta 0 ; \text { INA22::kanMX4; } \\
\text { GPD:Atp23; atp23::ATP23 }{ }^{H 128 A} \text {-URA3 }\end{array}$ & This study \\
\hline Ina22个ina17 $\Delta$ & $\begin{array}{l}\text { MATa; his3 } \Delta 1 ; \text { leu2 } \Delta 0 ; \quad \text { met15 } 00 ; \text { ura3 } \Delta 0 ; \quad \text { GPD:Ina22; } \\
\text { INA17::kanMX4 }\end{array}$ & This study \\
\hline $\operatorname{Ina} 22^{\mathrm{HA}}$ & $\begin{array}{l}\text { МАTa; his3 } \Delta 1 ; \text { leu2 } \Delta 0 ; \text { met15 } \Delta 0 ; \text { ura3 } \Delta 0 ; \text { ina22::INA22 }{ }^{H A} \text { - } \\
\text { HIS3MX6 }\end{array}$ & $\begin{array}{l}\text { Lytovchenko } \\
\text { et al., } 2014\end{array}$ \\
\hline
\end{tabular}


Table 5.7 - Continued from previous page

\begin{tabular}{|c|c|c|c|}
\hline Strain & \multicolumn{2}{|l|}{ Genotype } & \\
\hline $\operatorname{Atp} 23^{\mathrm{HA}}$ & $\begin{array}{l}\text { MATa; his3 } 1 ; \text { leu2 } \Delta 0 ; \text { met } 15 \Delta 0 ; \text { ura3 } \Delta 0 ; \text { atp } \\
\text { HIS3MX6 }\end{array}$ & $T^{T P 23^{H A}}-$ & This study \\
\hline Ina $22^{\text {FLAG }}$ & $\begin{array}{l}\text { MATa; his3 } \Delta 1 ; \text { leu2 } \Delta 0 ; \text { met } 15 \Delta 0 ; \text { ura3 } \Delta 0 ; \text { ina22. } \\
\text { URA3 }\end{array}$ & $422^{F L A G}$ & This study \\
\hline Atp $10^{\text {ProtA }}$ & 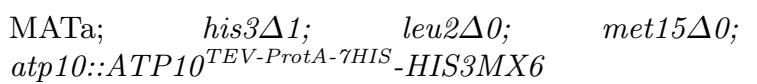 & ura3 $\Delta 0$ & This study \\
\hline Atp10 ${ }^{\text {ProtA }}$ ina22 $\Delta$ & 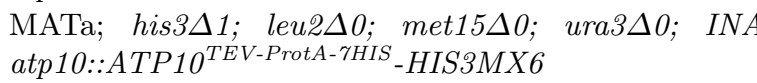 & $\operatorname{kanM} X_{4}$ & This study \\
\hline atp22 $\Delta$ & MATa; his3 $\Delta 1$; leu2 $\Delta 0$; met $15 \Delta 0$; ura3 $\Delta 0 ;$ ATP22: & $M X 6$ & This study \\
\hline $\mathrm{Cbp} 3^{\text {ProtA }}$ & 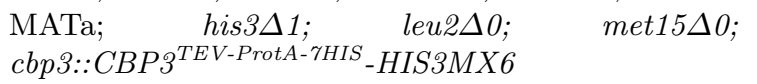 & ura3 $\Delta 0$ & This study \\
\hline $\mathrm{Cbp} 4^{\text {ProtA }}$ & $\begin{array}{lcc}\text { MATa; } & \text { his3 } 31 ; & \text { leu2 } \Delta 0 \\
\operatorname{cbp} 4: \therefore C B P 4^{T E V-P r o t A-7 H I S} & -H I S 3 M X 6\end{array}$ & ura $3 \Delta 0 ;$ & This study \\
\hline $\mathrm{Cbp} 6^{\text {ProtA }}$ & $\begin{array}{lcc}\text { MATa; } & \text { his3 } 31 ; & \text { leu2 } \Delta 0 ; \\
\operatorname{cbp} 6:: C B P 6^{\text {TEV-ProtA-7HIS }}-H I S 3 M X 6\end{array}$ & ura3 $\Delta 0$ & This study \\
\hline $\operatorname{cbp} 6 \Delta$ & MATa; his3 $\Delta 1$; leu2 $\Delta 0$; met $15 \Delta 0$; ura3 $\Delta 0 ; C B P 6:: H$ & MX6 & This study \\
\hline$q \operatorname{cr} 8 \Delta$ & MATa; his3 $\Delta 1 ;$ leu2 $\Delta 0 ;$ met $15 \Delta 0 ;$ ura3 $\Delta 0 ; Q C R 8:: H$ & MX6 & This study \\
\hline $\operatorname{cbp} 1 \Delta$ & $\begin{array}{l}\text { MATa leu2-3, 112 ura3-52 lys2 arg8:hisG } \\
\text { cox2::COX1 COX2] cbp1::kanMX4 }\end{array}$ & $\because A R G 8^{m}$ & This study \\
\hline Cbs1 $1^{\text {ProtA }}$ & $\begin{array}{l}\text { MATa; } \quad \text { his3 } \Delta 1 ; \quad \text { leu2 } \Delta 0 \\
\text { cbs1::CBS1 }\end{array}$ & ura3 $\Delta 0$ & This study \\
\hline $\mathrm{Cbs} 2^{\text {ProtA }}$ & $\begin{array}{lcr}\text { MATa; } & \text { his } 3 \Delta 1 ; & \text { leu2 } \Delta 0 ; \\
\text { cbs2::CBS2 } & \text { TEV-ProtA-7HIS } \\
\text {-HIS3MX6 }\end{array}$ & ura $3 \Delta 0$ & This study \\
\hline
\end{tabular}

For yeast strain generation, the corresponding chromosomal integration cassettes were used (Table 5.8) according to published procedures (Janke et al., 2004; Gueldener et al., 2002). For protein tagging, we used cassettes encoding the required C-terminal tag, allowing expression of the protein of interest from its endogenous promoter. For gene deletion, the corresponding genes were replaced by a selectivity marker. For gene overexpression, the endogenous promoter was replaced by the glyceraldehyde 3-phosphate dehydrogenase promoter, allowing an increase in protein amount.

For generation of Atp23 $3^{\mathrm{H} 128 \mathrm{~A}}$ catalytic mutant, a wild type ATP23 gene was first cloned into p426TEF vector and a point mutation was introduced via a sitedirected mutagenesis with oligonucleotides 5'-AATTGCTTATTTCGATGATCTAAAATGG-3' and 5'-AATTCATGCGAAAGCGTATCCTC-3'. Then, a fragment containing ATP23 $3^{H 128 A}$ and URA3 genes was amplified from this plasmid and used for homologous recombination to exchange the wild-type ATP23 with ATP23H128A in Atp23个 strain.

For Ina22 C-terminal tagging with a FLAG-tag, the integration cassette was amplified from pUG72 plasmid with primers 5'-GCTTGCGTTATATTTACATGTGGTATATCCGGATGCATAGAGCCTAATCGATGAATTCGAGCTCG-3' and 5'-GATAAAATCCCAAAATTTCTAGACTACAAAGACGATGACGACAAGTAGCGTACGCTGCAGGTCGAC-3', encoding a FLAG peptide, and the obtained PCRproduct was used for yeast transformation. 


\subsubsection{Oligonucleotides used in this study}

All nucleotides used in this study were ordered at Metabion (Martinsried, Germany) or SeqLab (Göttingen, Germany). The complete list is given in Table 5.10.

\subsubsection{Plasmids used in this study}

Plasmids, used in this study, are listed in Table 5.8. All plasmids were propagated in E. coli XL1 blue strain and prepared according to 5.5.2.

Table 5.8 Plasmids

\begin{tabular}{|c|c|c|}
\hline Plasmid & Purpose & Reference \\
\hline pFA6a-kanMX4 & yeast gene deletion & Janke et al., 2004 \\
\hline pFA6a-HIS3MX6 & yeast gene deletion & Janke et al., 2004 \\
\hline pYM2 & C-terminal tagging with $3 \mathrm{HA}$ & Janke et al., 2004 \\
\hline pYM10 & C-terminal tagging with TEV-ProA-7His & Janke et al., 2004 \\
\hline pYM-N14 & yeast protein overexpression & Janke et al., 2004 \\
\hline pYM-N15 & yeast protein overexpression & Janke et al., 2004 \\
\hline pUG72 & yeast gene deletion & Gueldener et al., 2002 \\
\hline p426TEF & yeast protein overexpression in yeast & Promega \\
\hline pTNT & in vitro transcription/translation & Promega \\
\hline pPROEXHTc & E. coli expression plasmid & Life Technologies (Invitrogen) \\
\hline
\end{tabular}

\subsubsection{E. coli strains used in this study}

E. coli strains used in this study are listed in table 5.9

Table 5.9 Bacterial strains

\begin{tabular}{l|l|l}
\hline \hline & & \\
Strain & Genotype & Reference \\
\hline \hline BL21(DE3) & fhuA2 [lon] ompT gal dcm hsdS $\lambda(\mathrm{DE} 3)\left(\mathrm{r}_{\mathrm{B}}^{-} \mathrm{m}_{\mathrm{B}}^{-}\right) \mathrm{F}^{-}$ & Stratagene \\
XL1 Blue & recA1 endA1 gyrA96 thi-1 hsdR17 supE44 relA1 lac [F'proAB & Stratagene \\
& lacI $\left.Z \Delta M 15 \operatorname{Tn} 10\left(\mathrm{Tet}^{\mathrm{r}}\right)\right]$ & \\
\hline \hline
\end{tabular}


Table 5.10 Oligonucleotides

\begin{tabular}{|c|c|}
\hline Purpose & Oligonucleotides \\
\hline INA22 deletion in BY4741 & $\begin{array}{l}\text { 5'-CAACAAGGAAAGACAAGTCATACGTAAAAGGTGTAAGGAAAAATGCGTACGCTGCAGGTCGAC-3'; } \\
\text { 5'-GCGTTATATTTACATGTGGTATATCCGGATGCATAGAGCCTACTAATCGATGAATTCGAGCTCG-3' }\end{array}$ \\
\hline INA17 deletion in BY4741 & $\begin{array}{l}\text { 5'-GTAAACTTTGAGAAAGGAAATGGGAACCAAAGCATATAATAGACAATATGCGTACGCTGCAGGTCGAC-3'; } \\
\text { 5'-CAATGGTACATTCTAGAAACACGAAAAGCCAACAAGATAACTAATTCTCAATCGATGAATTCGAGCTCG-3' }\end{array}$ \\
\hline Ina22 C-terminal HA-tagging & $\begin{array}{l}\text { 5'-TTGAATGAAATCGCCAAAGAACATGATAAAATCCCAAAATTTCTACGTACGCTGCAGGTCGAC-3'; } \\
\text { 5'-GCGTTATATTTACATGTGGTATATCCGGATGCATAGAGCCTACTAATCGATGAATTCGAGCTCG-3' }\end{array}$ \\
\hline Ina22 genomic overexpression & $\begin{array}{l}\text { 5'-CAACAAGGAAAGACAAGTCATACGTAAAAGGTGTAAGGAAAAATGCGTACGCTGCAGGTCGAC-3'; } \\
\text { 5'-GCTCCTTAAAAATAGACCATTTCTTAACACTTGTCTTGCCATAAACATCGATGAATTCTCTGTCG-3' }\end{array}$ \\
\hline $\begin{array}{l}\text { Ina22 C-terminal FLAG- } \\
\text { tagging }\end{array}$ & $\begin{array}{l}\text { 5'-GCTTGCGTTATATTTACATGTGGTATATCCGGATGCATAGAGCCTAATCGATGAATTCGAGCTCG-3'; } \\
\text { 5'-GATAAAATCCCAAAATTTCTAGACTACAAAGACGATGACGACAAGTAGCGTACGCTGCAGGTCGAC-3' }\end{array}$ \\
\hline Ina $22^{1-168}$ generation & $\begin{array}{l}\text { 5'-GAAAAAACTCCAGATGCTGGCGTTAAGAACAAAGAAGGGATTGTCACTTAGCGTACGCTGCAGGTCGAC-3'; } \\
\text { 5'-GCGTTATATTTACATGTGGTATATCCGGATGCATAGAGCCTACTAATCGATGAATTCGAGCTCG-3' }\end{array}$ \\
\hline Ina $22^{1-188}$ generation & $\begin{array}{l}\text { 5'-GTTCTAAAAGATTTAGAAAAGTCGAAAAAAGAAGATGAAAAGGTTTACCTATAGCGTACGCTGCAGGTCGAC- } \\
\text { 3'; 5'-GCGTTATATTTACATGTGGTATATCCGGATGCATAGAGCCTACTAATCGATGAATTCGAGCTCG-3' }\end{array}$ \\
\hline Ina22 $27-216$ mRNA synthesis & $\begin{array}{l}\text { 5'-GGATTTAGGTGACACTATAGAATACATGGTTGCATCAGCAAATGCGGG-3'; } \\
\text { 5'-GAGCCTACTACATCATCATTAGAAATTTTGGG-3' }\end{array}$ \\
\hline Ina22 $2^{27-206}$ mRNA synthesis & $\begin{array}{l}\text { 5'-GGATTTAGGTGACACTATAGAATACATGGTTGCATCAGCAAATGCGGG-3'; } \\
\text { 5'-CTACTACATCATCATGGCGATTTCATTCAAATCACC-3' }\end{array}$ \\
\hline Ina $22^{27-196}$ mRNA synthesis & $\begin{array}{l}\text { 5'-GGATTTAGGTGACACTATAGAATACATGGTTGCATCAGCAAATGCGGG-3'; } \\
\text { 5'-CTACTACATCATCATCATCATCATATGGACAT-3' }\end{array}$ \\
\hline Ina22 $2^{27-186}$ mRNA synthesis & $\begin{array}{l}\text { 5'-GGATTTAGGTGACACTATAGAATACATGGTTGCATCAGCAAATGCGGG-3'; } \\
\text { 5'-CTACTACATCATCATAACCTTTTCATCTTCTTTTTTC-3' }\end{array}$ \\
\hline Ina22 $2^{27-176}$ mRNA synthesis & $\begin{array}{l}\text { 5'-GGATTTAGGTGACACTATAGAATACATGGTTGCATCAGCAAATGCGGG-3'; } \\
\text { 5'-CTACTACATCATCATTAAATCTTTTAGAACATTC-3' }\end{array}$ \\
\hline
\end{tabular}


Table 5.10 - Continued from previous page

\begin{tabular}{|c|c|}
\hline Purpose & Primers \\
\hline Ina22 27-166 mRNA synthesis & $\begin{array}{l}\text { 5'-GGATTTAGGTGACACTATAGAATACATGGTTGCATCAGCAAATGCGGG-3'; } \\
\text { 5'-CTACTACATCATCATAATCCCTTCTTTGTTCTTAACGC-3' }\end{array}$ \\
\hline Ina $22^{27-156}$ mRNA synthesis & $\begin{array}{l}\text { 5'-GGATTTAGGTGACACTATAGAATACATGGTTGCATCAGCAAATGCGGG-3'; } \\
\text { 5'-CTACTACATCATCATTGGAGTTTTTTCGATTACTTC-3' }\end{array}$ \\
\hline Ina $22^{27-146}$ mRNA synthesis & $\begin{array}{l}\text { 5'-GGATTTAGGTGACACTATAGAATACATGGTTGCATCAGCAAATGCGGG-3'; } \\
\text { 5'-CTACTACATCATCATCTCGTCTATTATACTTTGCC-3' }\end{array}$ \\
\hline Ina22 $2^{27-136}$ mRNA synthesis & $\begin{array}{l}\text { 5'-GGATTTAGGTGACACTATAGAATACATGGTTGCATCAGCAAATGCGGG-3'; } \\
\text { 5'-CTACTACATCATCATCAATGACTCTTCCTTAGGAACC-3' }\end{array}$ \\
\hline ATP 4 coning into pTNT & $\begin{array}{l}\text { 5'-ATATGAATTCCCATGAGCATGAGTATGGGTGTCC-3'; } \\
\text { 5'-ATATGCGGCCGCTTA TTACATCATCATCTTCAATTTAGAAAGCAATTG-3' }\end{array}$ \\
\hline $\begin{array}{l}\text { ATP4 in vitro transcrip- } \\
\text { tion/translation }\end{array}$ & 5'-GGATTTAGGTGACACTATAGAATAC ATGAGCATGAGTATGGGTGTCCGTG-3'; \\
\hline Atp10 C-terminal tagging & $\begin{array}{l}\text { 5'-GCCAAATAGCCGCCCATCCCTTGTTGGCCGCCGCACAAAGCGTCAACTTCA ATCGATGAATTCGAGCTCG- } \\
\text { 3'; } \\
\text { 5'-GGCTACTCCATCTGAAAAGGAAGCATTGTGGAAGTTTGCCAAACGTCTGCGTACGCTGCAGGTCGAC-3' }\end{array}$ \\
\hline ATP10 mRNA synthesis & $\begin{array}{l}\text { 5'-GGATTTAGGTGACACTATAGAATACATGCAGGGCACTTTTAAAAGGTTTTACCATCCC-3'; } \\
\text { 5'-TTACATCATCATCAGACGTTTGGCAAACTTCCACAATGCTTCCTTTTC-3' }\end{array}$ \\
\hline ATP22 deletion & $\begin{array}{l}\text { 5'-GGGCTTTCAAATCTTCCAAGCGTTCACTTTAATGTTCTTATTGGCATGCGTACGCTGCAGGTCGAC-3'; } \\
\text { 5'-CTATCTATGAATGAATATTTACTATTTACTAGTGCTCATCTGGATATTAATCGATGAATTCGAGCTCG-3' }\end{array}$ \\
\hline Atp23 genomic overexpression & $\begin{array}{l}\text { 5'-GGAAAGCTGATAGTACCGAATTTTTTTTTTTTTTGGCACGATATGCGTACGCTGCAGGTCGAC-3'; } \\
\text { 5'-GCATGGTCCGTCTCCACCACTCAAACCCAGCATTATCCCCACTGCTATTCATCGATGAATTCTCTGTCG-3' }\end{array}$ \\
\hline ATP23 cloning into p426 TEF & $\begin{array}{l}\text { 5'-ATTATAGGATCCATGAATAGCAGTGGGGATAAT-3'; } \\
\text { 5'-AATAATAAGCTTTCATCTGTAAATCTCATCAAACG-3' }\end{array}$ \\
\hline ATP23 H128A mutagenesis & $\begin{array}{l}\text { 5'-AATTCATGCGAAAGCGTATCCTC-3'; } \\
\text { 5'-AATTGCTTATTTCGATGATCTAAAATGG-3' }\end{array}$ \\
\hline $\begin{array}{l}\text { ATP23 exchange } \\
\text { ATP23 }\end{array}$ & $\begin{array}{l}\text { 5'-ATGAATAGCAGTGGGGATAATGCTGGGTTTGAGTGGTGGAGACGGACCATGC-3'; } \\
\text { 5'-TCATCTGTAAATCTCATCAAACGGTCTCGTATCGGCGAAGCAACTTTCTTTGAAATTTTTTTGATTCGG-3' }\end{array}$ \\
\hline
\end{tabular}

Continued on the next page 
Table 5.10 - Continued from previous page

\begin{tabular}{l} 
Purpose \\
\hline Atp23 C-terminal tagging
\end{tabular}

QCR8 deletion in BY4741

CBP6 deletion in BY4741

Cbp3 C-terminal tagging

Cbp4 C-terminal tagging

Cbp6 C-terminal tagging

INA22 mRNA synthesis

Cbs1 C-terminal tagging

Cbs2 C-terminal tagging

\section{Primers}

5'-GCGTCTATATATTTTCTATTATAGAATATTGTCATTTATTACATTGGTTCA ATCGATGAATTCGAGCTCG-3';

5'-GGGACAGTTGCTTCGCCGATACGAGACCGTTTGATGAGATTTACAGA CGTACGCTGCAGGTCGAC-3'

5'-CAAATAATCAACAAAACACTACACAAAAACTTCTACGATAATGCGTACGCTGCAGGTCGAC-3';

5'-GAAGATCAAAAGATCGATGTAATGTAGAGGCATGGAAACAATATTA ATCGATGAATTCGAGCTCG-3'

5'-GCTTACCAAGTTAAACTCCGTATTCCACAAGCAAGTGCCAAAATGCGTACGCTGCAGGTCGAC-3';

5'-GAATAAATATGTATTTACAAGCTTAGAAAATAATGTGCTCTTTAATCGATGAATTCGAGCTCG-3'

5'-CTAAACGAGCTAGTTTGTAACTTCAAAACTTATGAAAACACATCGATGAATTCGAGCTCG-3' ;

5'-CCTAAAACCTTACCAAGCGAGAGAAGTAGGCTGTCATATACAAACCGTACGCTGCAGGTCGAC-3'

5'-GCAAAAGTTCAAGCTGCCCTTCCTAATTGAGTGACCCGACCCATCTAATCGATGAATTCGAGCTCG-3' ;

5'-GGAAATAGTCCAGGATAAGCAGGTTAAAAGCTGGTGGCGCTTCTGGCGTACGCTGCAGGTCGAC-3'

5'-GAATAAATATGTATTTACAAGCTTAGAAAATAATGTGCTCTTTAATCGATGAATTCGAGCTCG-3' ;

5'-GGAAAGCTTATTTACTGCAATGAGAACTGTATTATTTGGTAAACGTACGCTGCAGGTCGAC-3'

; 5'-GGATTTAGGTGACACTATAGAATACATGTTTATGGCAAGACAAGTG-3'

5'-GAGCCTACTACATCATCATTAGAAATTTTGGG-3

5'-GCACTTTAGGATAGCAGTAGTTTGCATTTTGCACGTTTCCCTTTCCATGCAATGCGTACGCTGCAGGTCGAC$3^{\prime}$;

5'-GTTATTATTTGCTTTGTTATTTATATCGTAAATGTGCGTTTGGCCGTTCATATCGATGAATTCGAGCTCG-3' 5'-GTAAATAAAACAAAGGGATAACGGACAAAAAGGCATCGTTTCTTTTGTACTAATCGATGAATTCGAGCTCG$3^{\prime}$;

5'-CAATTATAAAAACTATATGCATACCTCTAATTAATTACCATTTGCGTAAATCA CGTACGCTGCAGGTCGAC-3' 5'-CATATATGAAAATTTAGGAATATCTATTGTATATACATGAAATTTCTTCA ATCGATGAATTCGAGCTCG-3'; 5'-GGGAAACAGTTTATTACTAAGCAAAAAGCACTAGATTATCATTACCTG CGTACGCTGCAGGTCGAC-3' 


\section{$5.2 \quad E$. coli methods}

\subsubsection{E. coli growth}

E. coli cultures were grown according to standard procedures using lysogeny broth (LB, $1 \% \mathrm{NaCl}, 0.5 \%$ yeast extract, 1\% tryptone) (Sambrook and Russell, 2001). Plates were supplemented with $15 \mathrm{~g} / \mathrm{L}$ agar. Antibiotic selection markers were used in the following concentrations: $0.1 \mathrm{~g} / \mathrm{L}$ ampicillin, $0.03 \mathrm{~g} / \mathrm{L}$ kanamycin. Cryo-stocks of $E$. coli were generated by mixing $800 \mu \mathrm{L}$ of a culture in selective LB medium with $200 \mu \mathrm{L}$ sterile $80 \%$ glycerol in a cryo vial and freezing it at $-80^{\circ} \mathrm{C}$.

\subsubsection{E. coli transformation}

Chemically-competent E. coli cells were prepared according to Hanahan, 1983. In brief, a $100 \mathrm{~mL}$ culture with $\mathrm{OD}_{600}$ of 0.6 was cooled down on ice and cells were harvested by centrifugation for $5 \mathrm{~min}$ at $3000 \mathrm{~g}$. The cell pellet was resuspended in $40 \mathrm{~mL}$ ice-cold buffer 1 (30 mM KAc, $100 \mathrm{mM} \mathrm{RbCl,} 10 \mathrm{mM} \mathrm{CaCl} 2,50 \mathrm{mM} \mathrm{MnCl}_{2}$, $15 \%$ glycerol, $\mathrm{pH} 5.8$ ), followed by a $5 \mathrm{~min}$ incubation on ice. After one more centrifugation step, cells were resuspended in buffer 2 containing $10 \mathrm{mM}$ MOPS, 75 $\mathrm{mM} \mathrm{CaCl} 2,10 \mathrm{mM} \mathrm{RbCl}, 15 \%$ glycerol, $\mathrm{pH} 6.5$, aliquoted and stored at $-80^{\circ} \mathrm{C}$.

For transformation, cell aliquots were defrosted on ice, mixed with 100 ng plasmid DNA or $10 \mu \mathrm{L}$ ligation mix, incubated for $30 \mathrm{~min}$ on ice and subjected to a heat-shock $\left(60 \mathrm{sec}\right.$ at $42^{\circ} \mathrm{C}$ ). Afterwards, cells were cooled down on ice and incubated for 1 hour at $37^{\circ} \mathrm{C}$ in $1 \mathrm{~mL} \mathrm{LB}$ medium. Then, the cells were pelleted for $2 \mathrm{~min}$, $3000 \mathrm{~g}$, resuspended in $100 \mu \mathrm{lB}$ and plated to LB plates containing an appropriate selectivity marker.

\subsection{S. cerevisiae cultivation and preparation of mitochon- dria}

\subsubsection{Cultivation of $S$. cerevisiae}

For cultivation of $S$. cerevisiae, YPD, YPG and YPGal media were used (see Table 5.2). Liquid cultures were grown at $30^{\circ} \mathrm{C}$ with shaking $(190 \mathrm{rpm})$. For the preparation of solid media, $2 \%(\mathrm{w} / \mathrm{v})$ agar was added to the respective media before autoclaving. For the cultivation of nourseothricin and geneticin 418 sulfate resistant strains on solid media, the antibiotic was added to the media after autoclaving, to the final concentration of $1 \mathrm{mg} / \mathrm{mL}$. Synthetic medium lacking histidine or uridine was used to cultivate strains bearing HIS3 and URA3 genes in BY4741 background.

Yeast strains were preserved as cryo-stocks by transferring material from an appropriate plate into cryo vials containing $1 \mathrm{~mL}$ of $2 x Y P A D$ with $15 \%$ glycerol and 
storing them at $-80^{\circ} \mathrm{C}$.

\subsubsection{Growth test}

To examine the viability of different yeast strains, growth tests on fermentable (YPD) and non-fermentable (YPG, YPGal) carbon sources were performed. $5 \mathrm{ml}$ of YPD medium were inoculated with yeast cells and grown overnight at $30^{\circ} \mathrm{C}$ with shaking (200 rpm). On the next day, cells were spun down (100 g, $5 \mathrm{~min}$ ), washed once with sterile water and adjusted to the final $\mathrm{OD}_{600}=1$. Serial 10 -fold dilutions of cells were prepared (range from $\mathrm{OD}_{600}=1$ to $\mathrm{OD}_{600}=0,00001$ ), and the cells were spotted on plates with corresponding media. The plates were grown at different temperatures $\left(24,30\right.$ or $\left.37^{\circ} \mathrm{C}\right)$ for 2 days for YPD medium and up to five days for YPG and YPGal media-containing plates.

\subsubsection{Yeast transformation}

For yeast transformation with exogenous DNA, the lithium acetate/PEG method was used (Gietz and Schiestl, 2007). Yeast strains were precultured in $10 \mathrm{ml}$ of (2x) YPAD medium (see Table 5.2) overnight at $30{ }^{\circ} \mathrm{C}$ with shaking. On the next day, the overnight culture was used to inoculate $50 \mathrm{ml}$ of $(2 \mathrm{x})$ YPAD medium to $\mathrm{OD}_{600}$ of approximately 0.6 and grown until $\mathrm{OD}_{600}$ reached 1.5-2.0. The cells were harvested by centrifugation (1000 g, $10 \mathrm{~min}$ ) and washed once with sterile water (1000 g, 10 min). The cell pellet was washed with $0.1 \mathrm{M}$ lithium acetate solution and resuspended in $2 \mathrm{ml} 0.1 \mathrm{M}$ lithium acetate. For further transformation steps, $100 \mu \mathrm{l}$ of cells prepared in this way were used. $300 \mu \mathrm{g}$ of purified exogenous DNA were added to $100 \mu \mathrm{l}$ of resuspended cells together with $120 \mu \mathrm{g}$ of carrier DNA, followed by a $30 \mathrm{~min}$ incubation at $30^{\circ} \mathrm{C}$ with shaking $(450 \mathrm{rpm})$. After this, $600 \mu \mathrm{l}$ of lithium acetate/PEG solution were added (see Table 5.2), cells were carefully mixed by pipetting and incubated for 1 more hour at $30^{\circ} \mathrm{C}, 450 \mathrm{rpm}$. Then, $68 \mu \mathrm{l}$ of DMSO were added, the cells were exposed to $42^{\circ} \mathrm{C}$ heat shock for $15 \mathrm{~min}$, harvested by centrifugation (100 g $2 \mathrm{~min}$ ), resuspended in $60 \mu \mathrm{l}$ of $1 \mathrm{M}$ sorbitol and plated on appropriate solid selective media. Cells transformed with an antiobiotic-resistance cassette were grown additionally for 4 hours at $30^{\circ} \mathrm{C}$ in $1 \mathrm{~mL} 2 \mathrm{xYPAD}$, cells were collected by centrifugation and plated on appropriate selective plates.

\subsubsection{Preparation of mitochondria}

Mitochondria from yeast cells were prepared by differential centrifugation as described in Meisinger et al., 2006. For the mitochondrial preparation, yeast were pre-cultured in $5 \mathrm{~mL}$ of YPD media and then sequentially in 100 and $200 \mathrm{~mL}$ of either YPG or YPGal. The last pre-culture was used to inoculate the main culture. Flasks were incubated overnight at $30^{\circ} \mathrm{C}$ with shaking $(190 \mathrm{rpm})$, until $\mathrm{OD}_{600}$ 
reached 1.5 - 2.5. Cells were pelleted at $4000 \mathrm{~g}$ for $15 \mathrm{~min}$, washed with water and resuspended in DTT buffer $(2 \mathrm{ml}$ of buffer per $1 \mathrm{~g}$ of initial cell pellet, see Table 5.2 ), followed by a $30 \mathrm{~min}$ incubation at $30{ }^{\circ} \mathrm{C}$ with shaking $(200 \mathrm{rpm})$. Then the cells were collected by centrifugation (3000 g $10 \mathrm{~min}$ ), washed with $1.2 \mathrm{M}$ sorbitol and zymolyase buffer and resuspended in zymolyase buffer $(7 \mathrm{ml}$ of buffer per $1 \mathrm{~g}$ of initial cell pellet). Yeast cells were treated with $1 \mathrm{mg} / \mathrm{g}$ of zymolyase for 1 hour at $30^{\circ} \mathrm{C}$ with shaking $(190 \mathrm{rpm})$, to digest the cell wall and to obtain spheroplasts. Treated cells were washed once with zymolyase buffer without the enzyme, resuspended in homogenization buffer $(7 \mathrm{ml} / \mathrm{g}$ of starting yeast pellet) and homogenized by 15 strokes at $800 \mathrm{rpm}$ in Potter $\mathrm{S}$ glass-Teflon homogenizer. Then the homogenized cells were subjected to several centrifugations to remove unbroken cells and cellular debris: $5 \mathrm{~min}$ at $3000 \mathrm{rpm}$, then $10 \mathrm{~min}$ at $4000 \mathrm{rpm}$ and, finally, $15 \mathrm{~min}$ at $12000 \mathrm{rpm}$ to pellet the mitochondrial fraction (centrifugation was performed in Sorvall ${ }^{\circledR}$ RC6 Plus centrifuge, with Sorvall ${ }^{\circledR}$ F10S rotor). The mitochondrial pellet was washed once with SEM/PMSF buffer and resuspended in approximately $0.5 \mathrm{ml}$ of SEM/PMSF buffer. The protein concentration was determined by a Bradford assay (see 5.4.1) and the mitochondrial suspension was diluted to obtain final protein concentration $10 \mathrm{mg} / \mathrm{ml}$. Prepared mitochondria were aliquoted in single-use aliquots, flash-frozen and stored at $-80^{\circ} \mathrm{C}$.

\subsubsection{S. cerevisiae whole-cell extract preparation}

To prepare a whole cell extract, yeast were first precultured in YPD medium for few hours. An amount of cells corresponding to $3 \mathrm{OD}_{600}$ was taken. The cells were spun down for $5 \mathrm{~min}$ at $3000 \mathrm{~g}$ and resuspended in $275 \mathrm{mM} \mathrm{NaOH}$ with $1 \%$ $\beta$-mercaptoethanol, followed by 15 min incubation on ice. Afterwards, proteins were precipitated with $12 \%$ TCA for 30 min on ice and samples were centrifuged for 10 min at $16000 \mathrm{~g}$. The pellet was washed with ice-cold acetone, resuspended in SDSloading buffer and titrated with Tris base until the color of the sample turned blue. After this, samples were incubated at $37^{\circ} \mathrm{C}$ with shaking for at least $15 \mathrm{~min}$ and used for further SDS-PAGE analysis.

\subsection{Methods for analysis of proteins and protein complexes}

\subsubsection{Determination of protein concentration}

To determine protein amounts in isolated mitochondria, Roti ${ }^{\circledR}$-Quant Bradford protein determination assay was used. First, 0, 7.5, 15, 30 and $60 \mu \mathrm{g} / \mu \mathrm{l}$ bovine immunoglobulin was used to establish a calibration curve. Then 5, 10 or $20 \mu \mathrm{l}$ of mitochondrial suspension were adjusted to a volume of $84 \mu \mathrm{l}$ and $1 \mathrm{ml}$ of Roti ${ }^{\circledR}$ Quant reagent was added. After a 5 min incubation at room temperature, the 
optical density was measured at $595 \mathrm{~nm}$. The protein concentration was determined according to the calibration curve and adjusted to the concentration of $10 \mathrm{mg} / \mathrm{ml}$ with SEM buffer.

\subsubsection{SDS-PAGE}

Electrophoretic separation of proteins under denaturing conditions (Laemmli, 1970) was performed using polyacrylamide gels of different percentages with $0.1 \%$ SDS. For a stacking gel, a $4 \%$ polyacrylamide gel buffered with Tris/HCl pH 6.8 was used. For the separation of proteins, we used 10-17.5\% resolving acrylamide gels, buffered with Tris/HCl $\mathrm{pH}$ 8.8. Protein samples were loaded on a gel after mixing with SDS-loading buffer and a 5 min incubation at $95^{\circ} \mathrm{C}$, or after a 15 min agitation at room temperature. Gels were run at $30 \mathrm{~mA} /$ gel in SDS-running buffer containing $1 \%$ SDS. For the estimation of protein electromobility, SDS-PAGE Molecular Weight Standart, Broad Range (Bio-Rad) or unstained protein standard from Serva was used.

\subsubsection{Urea SDS-PAGE}

For separation of small proteins and mitochondrial-encoded products, proteins were analyzed by $17.5 \%$ Urea SDS-PAGE. In brief, proteins were stacked in a stacking gel containing 5.45\% Urea acrylamide solution, $0.1 \mathrm{M}$ Tris/HCl pH 6.8, 3.64 M Urea and $0.1 \%$ SDS. For the separation of proteins, the resolving gel was used, containing $17.5 \%$ of Urea acrylamide solution, $0.68 \mathrm{M}$ Tris/ $\mathrm{HCl} \mathrm{pH} \mathrm{8.8,} 8 \mathrm{mM} \mathrm{NaCl}$, $5.43 \mathrm{M}$ Urea and 0.1\% SDS. Samples were prepared as in 5.4.2, loaded on a gel and the gel was run at $35 \mathrm{~mA} /$ gel for 5 hours in Urea running buffer $(0.1 \%$ SDS, $50 \mathrm{mM}$ Tris, $192 \mathrm{mM}$ glycine).

\subsubsection{BN-PAGE}

For separation of large mitochondrial protein complexes in native conditions, BN-PAGE electrophoresis was used (Schägger and Jagow, 1991). Mitochondrial samples were pelleted $\left(16000 \mathrm{~g}, 10 \mathrm{~min}, 4^{\circ} \mathrm{C}\right)$ and solubilized to the final protein concentration $1 \mu \mathrm{g} / \mu \mathrm{L}$ for $15 \mathrm{~min}$ at $4^{\circ} \mathrm{C}$. Unsolubilized material was removed by a clarifying spin $\left(10 \mathrm{~min}, 16000 \mathrm{~g}, 4^{\circ} \mathrm{C}\right)$, supernatant was mixed with $10 \mathrm{X}$ Blue Native loading buffer and loaded onto a gradient gel of desired percentage with $4 \%$ stacking gel. Electrophoresis was performed using the SE600 Ruby System (GE-Healthcare), at $4^{\circ} \mathrm{C}$, starting with the voltage of $200 \mathrm{~V}, 15 \mathrm{~mA}$ for $1 \mathrm{~h}$, and then shifted to 600 V. High molecular weight calibration kit (GE Healthcare) was used to estimate the molecular weight of protein complexes. For the first 2 hours, cathode buffer with Coomassie Brilliant Blue G-250 was used. Afterwards, the cathode buffer was 
exchanged toa Coomassie-free buffer and the gel was run until the running front reached the gel bottom.

For analysis of protein complexes by second-dimension electrophoresis, a gel strip was cut out of a Blue Native gel and assembled on top of an SDS-PAGE stacking gel. The gel was run according to 5.4.2.

Solubilization buffer: $20 \mathrm{mM}$ Tris/HCl (pH 7.4), $0.1 \mathrm{mM}$ EDTA (pH 8.0), 50 $\mathrm{mM} \mathrm{NaCl}, 10 \%$ glycerol, $1 \mathrm{mM}$ PMSF, supplemented with $1 \%(\mathrm{w} / \mathrm{v})$ digitonin or $0.6 \%(\mathrm{v} / \mathrm{v})$ dodecylmaltoside (DDM).

\subsubsection{Western blotting and immunodecoration}

Proteins separated on SDS-PAGE or BN-PAGE gels were transferred to PVDF membranes by semi-dry blotting using PEQLAB chambers. A membrane of appropriate size was activated in methanol and assembled underneath the gel, between layers of Whatman paper soaked in blotting buffer. Transfer to the membrane was performed at $250 \mathrm{~mA}$ for 2.5 hours. After the transfer, the membrane was stained in Coomassie staining solution to visualize protein bands and protein molecular weight marker. The membrane was cut in appropriate parts, the Coomassie dye was removed by washing with methanol and the membrane was incubated for 1 hour in $5 \%$ milk in TBST (for SDS-PAGE gels) or 2 hours in 10\% milk in TBST (for BN-PAGE gels) for blocking. After this, membranes were incubated with primary antibodies for 1 hour at room temperature or at $4^{\circ} \mathrm{C}$ overnight. Membranes were washed with TBST buffer 3 times for 5 minutes, followed by 1 hour incubation with secondary antibodies, coupled to HRP. After 3 more washing steps, signals were detected with the help of enhanced chemiluminescence detection kit (GE Healthcare) using X-ray films.

\subsubsection{Coomassie Brilliant Blue staining}

Proteins in acrylamide gels and on PVDF-membranes were stained with Coomassie staining solution (see Table 5.2) for 1 hour for gels and for a few minutes for membranes. After this, gels and membranes were destained in destaining solution until protein bands appeared. To remove the Coomassie dye, the membranes were washed with methanol.

\subsubsection{Colloidal Coomassie staining}

To avoid background staining of acrylamide gels, Colloidal Coomassie staining was used. In brief, a polyacrylamide gel was first fixed in a fixing solution (40\% ethanol, 10\% acetic acid) for at least $60 \mathrm{~min}$. Afterwards, the gel was briefly washed twice with distilled water. Following washing, the gel was stained in dye 
solution (20\% methanol, 0.08\% (w/v) Coomassie Brilliant Blue G-250, 1.6\% orthophosphoric acid, $8 \%$ (w/v) ammonium sulfate) for at least 24 hours. Then, Coomassie particles were removed by washing the gel in $1 \%$ acetic acid until the background disappeared.

\subsubsection{IgG affinity chromatography}

IgG affinity chromatography was used for isolation of protein complexes. For this, yeast strains were generated, in which the C-terminus of the protein of interest was fused to Protein A from S. aureus. IgG-sepharose beads were prepared by cross-linking purified human IgG to cyanobromide-activated Sepharose 4B (GE Healthcare) according to manufacturer's instructions. Before use, the beads were activated by washing twice with 5 bed volumes of acetate buffer $\mathrm{pH} 3.4$ (1.43 ml acetic acid was diluted with $50 \mathrm{ml}$ of water, pH was set to 3.4 with $3.8 \%$ (w/v) $\mathrm{NH}_{4} \mathrm{Ac}$ ). Then the beads were washed twice with $2 \mathrm{x}$ and $1 \mathrm{x}$ solubilization buffer. Mitochondrial membranes were solubilized as described in 5.6.1. Unsolubilized particles were removed by 10 minutes centrifugation at $16000 \mathrm{~g}$ and the supernatant was mixed with activated IgG sepharose. The mixture was incubated for 2 hours at $4^{\circ} \mathrm{C}$ with moderate shaking. After this, the unbound fraction was removed by centrifugation at $200 \mathrm{~g}$ for 30 seconds. Beads with bound proteins were washed 10 times with 5 bed volumes of washing buffer (30 seconds, $200 \mathrm{~g}$ ). For SDS-PAGE analysis, elution from the beads was performed with SDS-loading buffer. Approximately $40 \mu \mathrm{l}$ of SDS-loading buffer were added to the beads and the mixture was incubated at $37^{\circ} \mathrm{C}$ for 15 min with shaking. Eluted proteins were analyzed by SDS-PAGE, followed by immunoblotting. For isolation of native protein complexes, the protein A tag was cleaved by His-tagged tobacco etch virus (TEV) protease $(10 \mu \mathrm{g}$ protease/1 mg mitochondrial proteins) overnight at $4^{\circ} \mathrm{C}$. After cleavage, the protease was removed by adding Ni-NTA beads ( 1 hour incubation at $4^{\circ} \mathrm{C}$ ) and purified protein complexes were eluted by centrifugation (200 g, 2 minutes). For purification of Atp10 ${ }^{\text {ProtA }}$ containing protein complexes in low and high-stringent conditions, mitochondria were solubilized either in a low stringency (20 mM Tris/HCl pH 7.4, 10\% glycerol, $160 \mathrm{mM}$ $\mathrm{NaCl}, 2 \mathrm{mM} \mathrm{PMSF}, 1 \%$ digitonin) or in a high stringency buffer $(10 \mathrm{mM}$ Tris/HCl pH 9.0, $400 \mathrm{mM} \mathrm{NaCl}, 2 \mathrm{mM}$ PMSF, 3\% digitonin, $10 \mathrm{mM}$ Imidazol, according to Rak et al., 2011), respectively, as in 5.6.1. Supernatant was incubated with beads for 1.5 hours at $4^{\circ} \mathrm{C}$ and beads were washed with $0.3 \%$ digitonin-containing low and high-stringency buffers. All other steps were performed as in 5.4.8.

\subsubsection{Protein immunoprecipitation}

To immunoprecipitate endogenous yeast proteins, a corresponding specific antiserum was covalently coupled to Protein A CL 4B sepharose (GE Healthcare). 
For this, the sepharose was transferred to a Mobicol spin column and washed twice with $50 \mathrm{mM}$ phosphate buffer pH 7.4 for $30 \mathrm{sec}, 100 \mathrm{~g}$. After this, the specific serum was diluted 1:1 with a $50 \mathrm{mM}$ phosphate buffer $\mathrm{pH} 7.4$, added to the column and incubated with the protein A-coupled sepharose for 1 hour at room temperature, with shaking. Afterwards, columns were washed with $0.1 \mathrm{M}$ sodium borate (30 sec, 100g). To chemically crosslink Protein A to IgG, dimethyl pimelimidate (DMP) was diluted in sodium borate buffer to a final concentration of $5 \mathrm{mg} / \mathrm{mL}$ and added to the spin columns. The crosslinking reaction was performed for $30 \mathrm{~min}$ at room temperature. The columns were afterwards washed twice with $1 \mathrm{M}$ Tris/HCl pH 7.4 and further incubated in it for 2 hours at room temperature or overnight at $4^{\circ} \mathrm{C}$, with shaking. During the last step, non-crosslinked IgG was removed from the beads by washing them 3 times with 5 bed volumes of $0.1 \mathrm{M}$ glycine $\mathrm{pH} 2.6$. The columns were further washed 5 times with TBS buffer and stored in TBS with $1 \%$ sodium azide at $4^{\circ} \mathrm{C}$.

Mitochondria were solubilized as in 5.6.1 and isolation of proteins was performed as in 5.4.8. Bound proteins were eluted with $0.1 \mathrm{M}$ glycine $\mathrm{pH} 2.6$ and further analyzed.

\subsubsection{FLAG-affinity purification}

To perform a native isolation of protein complexes, Ina22 was C-terminally tagged with a FLAG peptide. To purify Ina22-containing complexes of interest, Anti-FLAG M2 Affinity Gel (Sigma-Aldrich) was used. In brief, mitochondria were solubilized according to 5.6.1, unsolubilized material was removed by centrifugation for $10 \mathrm{~min}$ at $16000 \mathrm{~g}, 4^{\circ} \mathrm{C}$. The obtained supernatant was mixed with anti-FLAG beads in ratio $10 \mu \mathrm{L}$ of beads $/ 1 \mathrm{mg}$ of mitochondrial proteins and incubated on the rotation wheel for 2 hours at $4^{\circ} \mathrm{C}$. The unbound material was removed by 30 sec centrifugation at $200 \mathrm{~g}$ and beads were washed 10 times with 5 bed volumes of washing buffer. Bound proteins were eluted with either $0.1 \mathrm{M}$ glycine $\mathrm{pH} 2.6$ (for further SDS-PAGE analysis) or with the FLAG-peptide (for further BN-PAGE analysis of isolated protein complexes).

\subsubsection{In vivo labeling of mitochondrial-encoded proteins}

For in vivo labeling of mitochondrial-encoded proteins with $\left[{ }^{35} \mathrm{~S}\right] \mathrm{Met}$, yeast cells were grown overnight in YPG medium at $30^{\circ} \mathrm{C}$ with agitation $(200 \mathrm{rpm})$. The next morning, yeast cultures were diluted to $\mathrm{OD}_{600}$ of 0.4 and grown in YPG until $\mathrm{OD}_{600}$ reached approximately 1 . After this, the amount of cells corresponding to $0.3 \mathrm{OD}_{600}$ was taken, cells were pelleted by centrifugation ( $2 \mathrm{~min} 3000 \mathrm{~g}$, room temperature) and washed once with labeling buffer $(50 \mathrm{mM}$ phosphate buffer $\mathrm{pH}$ 7.2, $2 \%$ galactose). Afterwards, cells were resuspended in $0.5 \mathrm{~mL}$ of labeling buffer 
and incubated at $30^{\circ} \mathrm{C}$ for $10 \mathrm{~min}, 600 \mathrm{rpm}$. Next, cycloheximide was added to the final concentration of $150 \mu \mathrm{g} / \mathrm{mL}$ and cells were incubated for 5 more minutes. To start labeling of mitochondrial-encoded proteins, $20 \mu \mathrm{C}_{\mathrm{i}}$ of $\left.{ }^{35} \mathrm{~S}\right]$ Met were added and labeling was performed for the required time period. Labeling was stopped by addition of $10 \mathrm{mM}$ unlabeled methionine and $100 \mu \mathrm{g} / \mathrm{mL}$ chloramphenicol. To address protein stability, samples were incubated at $30^{\circ}$ for additional 30, 60 or 90 minutes. Cells were spun down and washed once with water. Cell pellet was resuspended in $500 \mu \mathrm{L}$ of water and yeast whole-cell lysate was prepared (see 5.3.5). Proteins were precipitated with TCA and analyzed by SDS-PAGE or Urea SDSPAGE, Western blotting and digital autoradiography.

\subsubsection{TCA protein precipitation}

For protein precipitation, TCA and sodium desoxycholate were added to the protein sample to reach final concentrations of $14 \%$ and $0.0125 \%$, respectively. The samples were incubated at $4^{\circ} \mathrm{C}$ for 30 minutes. After this, the samples were centrifuged at $4^{\circ} \mathrm{C}$ for 30 minutes to pellet the proteins. The pellet was washed with $500 \mu \mathrm{l}$ of ice-cold acetone, dried at $37^{\circ} \mathrm{C}$ for $10-15$ minutes, diluted in SDS-PAGE loading buffer and incubated for 15 minutes at $37^{\circ} \mathrm{C}, 900 \mathrm{rpm}$. The samples were loaded on SDS-PAGE or Urea SDS-PAGE and analyzed further.

\subsubsection{Digital autoradiography}

Radioactively labeled proteins in dried SDS-PAGE, BN-PAGE gels or on PVDF membranes were visualized by exposure to Storage Phosphor Screen and detecting signals with a Storm 820 Phosphorimager (GE Healthcare).

\subsection{Molecular biology techniques}

\subsubsection{Preparation of genomic DNA from S. cerevisiae}

To prepare genomic DNA from S. cerevisiae, yeast were precultured overnight in $5 \mathrm{ml}$ of YPD medium at $30^{\circ} \mathrm{C}$. On the next day, the cells were pelleted for $10 \mathrm{~min}$ at $3000 \mathrm{~g}$ and resuspended in $150 \mu \mathrm{l}$ of $50 \mathrm{mM}$ Tris/HCl pH 7.5, $10 \mathrm{mM}$ EDTA, 0.3\% $\beta$-mercaptoethanol, $0.5-0.25 \mathrm{mg} / \mathrm{ml}$ Zymolyase. The mixture was incubated at $37^{\circ} \mathrm{C}$ for 1 hour. After this, $20 \mu \mathrm{l}$ of $10 \%$ SDS and $100 \mu \mathrm{l}$ of $8 \mathrm{M}$ ammonium acetate were added, the solution was mixed and incubated at $-20^{\circ} \mathrm{C}$ for 15 minutes. Proteins were pelleted by a $15 \mathrm{~min}$ centrifugation at $14000 \mathrm{rpm}$, and $180 \mu \mathrm{l}$ of clear supernatant were taken. This supernatant was mixed with $180 \mu \mathrm{l}$ of isopropanol and spun down for $15 \mathrm{~min}, 14000 \mathrm{rpm}$ at $4^{\circ} \mathrm{C}$. The pellet was washed with $70 \%$ ethanol, dried and resuspended in $100 \mu$ l of nuclease-free water. DNA concentration was determined using NanoVue ${ }^{\mathrm{TM}}$ Spectrophotometer (GE Healthcare). 


\subsubsection{Plasmid isolation from $E$. coli}

Plasmid DNA was isolated using Wizard ${ }^{\circledR}$ Plus SV Minipreps DNA Purification System (Promega) according to manufacturer's instructions. In brief, $4 \mathrm{~mL}$ of $E$. coli culture were grown overnight at $37^{\circ} \mathrm{C}$ in LB medium supplemented with appropriate antibiotic. Next morning, the cells were harvested $(5000 \mathrm{~g}, 1 \mathrm{~min})$. The pellet was resuspended in $250 \mu \mathrm{L}$ resuspension buffer and $250 \mu \mathrm{L}$ lysis buffer were added together with $10 \mu \mathrm{L}$ alkaline protease. The samples were inverted 5 times and incubated at room temperature for 5 min. Afterwards, $350 \mu \mathrm{L}$ of neutralization buffer were added and the samples were centrifuged for $10 \mathrm{~min}$ at $16000 \mathrm{~g}$. The supernatant was collected, loaded on a filter column and centrifuged for $1 \mathrm{~min}$, $16000 \mathrm{~g}$. The filter columns were sequentially washed with 750 and $500 \mu \mathrm{L}$ of washing buffer and dried afterwards. DNA was eluted from the column with $100 \mu \mathrm{L}$ of $\mathrm{dH}_{2} \mathrm{O}$ by centrifugation for $1 \mathrm{~min}, 16000 \mathrm{~g}$. Plasmid concentration was determined using NanoVue ${ }^{\mathrm{TM}}$ Spectrophotometer (GE Healthcare) and DNA was stored at $-20^{\circ} \mathrm{C}$.

\subsubsection{Polymerase chain reaction (PCR)}

DNA fragments for molecular cloning and yeast transformation were amplified using KOD Hot Start DNA polymerase (Novagen) according to the manufacturer's protocol. Each $50 \mu \mathrm{l}$ reaction mix contained $100 \mathrm{ng}$ of genomic or plasmid DNA, $0.2 \mathrm{mM}$ dNTP, $1 \mathrm{mM} \mathrm{MgSO} 4,0.3 \mu \mathrm{M}$ forward and reverse primers and $1 \mathrm{U}$ KOD polymerase in $1 \mathrm{x}$ KOD reaction buffer. First, template DNA was denatured at $95^{\circ} \mathrm{C}$ for 2 minutes. After this, 35 cycles of polymerase chain reaction were performed, each consisting of $10 \mathrm{sec}$ primer annealing (temperature of annealing depended on primers) and $20 \mathrm{sec}$ elongation at $70^{\circ} \mathrm{C}$. PCR products were analyzed by agarose gel electrophoresis with ethidium bromide staining followed by exposure to UV-light.

\subsubsection{Agarose gel electrophoresis}

For the visualization and purification of PCR products of different length, agarose gel electrophoresis was used. $1 \%(\mathrm{w} / \mathrm{v})$ agarose solution was freshly prepared by dissolving an appropriate amount of dry agarose in TAE buffer. The mixture was heated until agarose dissolved completely and then cooled to approximately $50^{\circ} \mathrm{C}$. Ethidium bromide was added to the final concentration of $1 \mu \mathrm{g} / \mathrm{ml}$ and a solid gel was prepared. Samples were mixed with DNA loading dye, loaded on the solid gel and run at $8 \mathrm{~V} / \mathrm{cm}$. Separated DNA fragments were visualized by illuminating the gel with UV-light. 


\subsubsection{Purification of PCR products}

PCR products were purified using Wizard ${ }^{\circledR}$ Plus SV Gel and PCR purification system (Promega) according to the manufacturer's protocol. For this, a PCR product was mixed with an equal amount of Membrane Binding solution, the mixture was transferred to a Minicolumn and incubated for 1 minute at room temperature. The minicolumn was centrifuged for $1 \mathrm{~min}$ at $16000 \mathrm{~g}$ and washed with 700 and 500 $\mu$ l of Membrane Wash solution. DNA bound to the column was eluted with $50 \mu$ of DNase-free water. The final DNA concentration was estimated using NanoVue ${ }^{\mathrm{TM}}$ Spectrophotometer (GE Healthcare).

\subsubsection{Molecular cloning}

Molecular cloning was performed according to standard procedures (Sambrook and Russell, 2001). In brief, the plasmid of interest and purified PCR products were digested with the appropriate restriction enzymes (FastDigest, Fermentas). For this, $30 \mu \mathrm{L}$ reactions were prepared containing $1 \mathrm{x}$ FastDigest buffer, $1 \mu \mathrm{L}$ of each restriction enzyme and $500 \mathrm{ng}$ DNA. The reaction was incubated at $37^{\circ} \mathrm{C}$ for $30 \mathrm{~min}$ with slight agitation $(600 \mathrm{rpm})$. The cut plasmid was dephosphorylated with $1 \mu \mathrm{L}$ alkaline phosphatase in $1 \mathrm{x}$ phosphatase buffer. DNA was purified according to 5.5.5 and ligated. For the ligation reaction, $100 \mathrm{ng}$ of plasmid was mixed with insert in 1:3 molar ratio, and the reaction was carried out in a $1 x$ ligation buffer with $1 \mu \mathrm{L}$ T4 DNA ligase (Rapid DNA ligation Kit, Thermo Scientific) at $21^{\circ} \mathrm{C}$ for 1 hour. The ligation reaction was used to transform XL1 blue E. coli cells. All plasmids were sequenced by GATC Biotech AG, Konstanz, or SeqLab, Göttingen.

\subsubsection{In vitro mutagenesis}

For introducing a point mutation in the catalytic site of Atp23, the ATP23 gene was first cloned into the p426TEF plasmid. Afterwards, the plasmid was amplified with ATP23-specific forward and reverse primers. The forward primer contained a nucleotide exchange allowing the mutation of a Histidine residue to Alanine. In brief, the PCR reaction $(50 \mu \mathrm{L})$ contained $100 \mathrm{ng}$ plasmid DNA, $0.2 \mu \mathrm{M}$ forward and reverse primers, $1 \mu \mathrm{L}$ dNTP mix and $2 \mathrm{U}$ of polymerase in $1 \mathrm{x}$ buffer. The reaction was performed according to 5.5.3. After the reaction, the template plasmid was digested for 1 hour at $37^{\circ} \mathrm{C}$ with DpnI, then the DNA was purified and ligated (see 5.5.6).

\subsubsection{In vitro mRNA synthesis}

For in vitro mRNA synthesis, SP6 mMESSAGE mMACHINE ${ }^{\circledR}$ Kit was used according to the manufacturer's protocol. 0.1-0.2 $\mu \mathrm{g}$ of purified PCR products was 
incubated at $37^{\circ} \mathrm{C}$ for 2 hours in a $20 \mu$ reaction mixture containing $1 \mathrm{x}$ Reaction buffer, $1 x N T P / C a p$ and $2 \mu$ l enzyme mix. To recover the mRNA, $30 \mu$ of lithium chloride precipitation solution was added, mixed and incubated for $30 \mathrm{~min}$ at $-20^{\circ} \mathrm{C}$. Then the mRNA was pelleted for $30 \mathrm{~min}$ at $14000 \mathrm{rpm}$. The pellet was washed once with $70 \%$ ethanol and dried at room temperature for $15 \mathrm{~min}$. mRNA was diluted with approximately $25 \mu \mathrm{l}$ of RNAse-free water and the mRNA concentration was determined using NanoVue ${ }^{\mathrm{TM}}$ Spectrophotometer (GE Healthcare).

\subsubsection{Synthesis of radiolabeled protein precursors}

Mitochondrial protein precursors were translated in vitro in the presence of $\left.{ }^{35} \mathrm{~S}\right]$ methionine using TNT SP6 quick Coupled Transcription/Translation system (Promega) or Flexi ${ }^{\circledR}$ Rabbit Reticulocyte Lysate system (Promega). Reactions were assembled according to manufacturer's protocols and incubated for 1 hour 30 minutes at $30^{\circ} \mathrm{C} .2 \mu \mathrm{l}$ of plasmids containing the open reading frame of interest under the control of SP6 promoter or $1.5 \mu \mathrm{g}$ of mRNA were used per $50 \mu \mathrm{l}$ reaction volume for both systems, respectively.

\subsubsection{Determination of nucleic acid concentration}

mRNA or DNA concentrations were determined using NanoVue ${ }^{\mathrm{TM}}$ Spectrophotometer (GE Healthcare) according to the manufacturer's instruction.

\subsection{Assays with purified mitochondria}

\subsubsection{Solubilization of mitochondrial membranes}

Mitochondrial membranes were solubilized in solubilization buffer containing $1 \%$ digitonin or $0.6 \%$ DDM (see Table 5.2). For this, mitochondria were thawed on ice, pelleted by centrifugation (10 min, $16000 \mathrm{~g}$ ) and then resuspended at $1 \mathrm{mg} / \mathrm{ml}$ in solubilization buffer. The suspension was incubated for $15 \mathrm{~min}$ on a rotation wheel at $4^{\circ} \mathrm{C}$. Unsolubilized particles were pelleted by centrifugation (10 min, 16000 g), clear supernatant was collected and used for different purposes.

\subsubsection{Import of radiolabeled protein precursors into mitochondria}

For protein import into mitochondria, radiolabeled protein precursors were synthesized as described in 5.5.9. Mitochondria were thawed on ice and $65 \mu \mathrm{g}$ of mitochondria diluted in $100 \mu \mathrm{l}$ BSA import buffer were used per reaction. $12 \mathrm{mM}$ NADH and ATP were added to mitochondrial samples. $1 \mu \mathrm{l}$ of AVO mix was added to the control reaction to disrupt the membrane potential. The import reaction was started by addition of $10 \mu$ l of radiolabeled protein precursor and stopped by addition of 1 
$\mu \mathrm{l}$ AVO mix. Import was performed at $25^{\circ} \mathrm{C}$ for 4 different time points. After this, mitochondria were spun down for 10 minutes at $16000 \mathrm{~g}$, washed once with SEM buffer and treated with $1 \mathrm{mg} / \mathrm{ml}$ proteinase $\mathrm{K}$ if needed $(4 \mu \mathrm{lof} 1 \mathrm{mg} / \mathrm{ml} \mathrm{PK}$ was added per reaction followed by 15 minutes incubation on ice). The mitochondrial pellet was resuspended in $25 \mu \mathrm{l}$ of SDS-loading buffer and proteins were separated by SDS-PAGE electrophoresis. The gel was stained in Coomassie R-250 staining solution, dried and exposed on storage phosphor screens (GE Healthcare).

\subsubsection{In organello protein labeling with $\left[{ }^{35} \mathrm{~S}\right] \mathrm{Met}$}

For the analysis of mitochondrial translation products, proteins encoded in the mitochondrial genome were labeled with $\left[{ }^{35} \mathrm{~S}\right]$ methionine during mitochondrial translation. $40 \mu \mathrm{g}$ of thawed mitochondria were used per reaction. The reaction buffer contained $40 \mu \mathrm{l} 1.5 \mathrm{x}$ translation buffer (see 5.2), $1.5 \mu \mathrm{l}$ creatine kinase (1 $\mathrm{mg} / \mathrm{ml}$ ) and $12.5 \mu \mathrm{l}$ of water. Samples were prewarmed to $30^{\circ} \mathrm{C}$ and the reaction was started by adding $20 \mu \mathrm{M}$ of $\left[{ }^{35} \mathrm{~S}\right]$ methionine. Samples were incubated at $30^{\circ} \mathrm{C}$ during different pulse periods. The reaction was stopped by adding $5 \mu \mathrm{M}$ methionine and put on ice. In order to estimate the degradation of newly synthesized translation products, samples were left at $30^{\circ} \mathrm{C}$ for different time points. Then, the samples were spun down (10 minutes, $16000 \mathrm{~g}$ ) and mitochondrial pellets were washed once with SEM buffer. The final pellet was resuspended in $30 \mu \mathrm{l}$ of SDS-loading buffer and incubated at $37^{\circ} \mathrm{C}$ with shaking for 20 min. Samples were loaded on a $16 \%$ gel and proteins were separated by SDS-PAGE followed by transfer to a PVDF membrane. Membranes were stained in Coomassie staining solution to visualize protein marker, dried and exposed on a storage phosphor screen (GE Healthcare).

\subsubsection{Proteinase $\mathrm{K}$ protection assay}

To determine mitochondrial protein localization, a proteinase $\mathrm{K}$ protection assay was performed. For this, mitochondria were incubated for $20 \mathrm{~min}$ in $1 \mathrm{mg} / \mathrm{ml}$ either SEM, EM buffer or EM buffer with addition of $0.16 \%$ Triton-X100. Then each sample was treated for 10 minutes with either 10 or $20 \mu \mathrm{g}$ proteinase K. Proteinase $\mathrm{K}$ was inhibited by addition $1 \mathrm{mM}$ PMSF and samples were clarified by 10 minutes centrifugation at $14000 \mathrm{rpm}$. The pellet was resuspended in SDS-Loading buffer and analyzed using SDS-PAGE and western blotting.

\subsubsection{Carbonate extraction of proteins}

To determine the mode of protein association with the mitochondrial membrane, carbonate extraction was performed. Mitochondria were resuspended in 0.1 $\mathrm{M} \mathrm{Na}_{2} \mathrm{CO}_{3} \mathrm{pH} 11.5$ or sonicated in a buffer containing $500 \mathrm{mM} \mathrm{NaCl}, 0.125 \%$ TritonX100 and $20 \mathrm{mM}$ Tris/HCl pH 7.4 (40\% duty cycle, 1 minute, 5 cycles) followed by a 
20 minutes incubation on ice. After the incubation, samples were spun down for 30 minutes at $16000 \mathrm{~g}$. The supernatants were transferred to new eppendorf tubes and the pellets were resuspended in $100 \mu \mathrm{l}$ of SEM buffer. Proteins were precipitated with TCA as described in 5.4.12. The protein pellet was diluted in SDS-loading buffer, loaded on SDS-PAGE and the protein content of each fraction was analyzed by western blotting and immunodecoration.

\subsubsection{Determination of in-gel activity of $\mathrm{F}_{1} \mathrm{~F}_{\mathrm{o}}$-ATP synthase}

To perform in-gel activity assays for mitochondrial complex $\mathrm{V}$, mitochondria were first solubilized as described in 5.6.1 and protein complexes were separated using BN-PAGE (see 5.4.4). For the $\mathrm{F}_{1} \mathrm{~F}_{\mathrm{o}}$-ATP synthase activity determination, a gel strip was cut from the BN-PAGE gel and incubated for $30 \mathrm{~min}$ in complex $\mathrm{V}$ calibration buffer (35 mM Tris, $220 \mathrm{mM}$ glycine $\mathrm{pH} 8.3$ ). After this, the gel was transferred to complex $\mathrm{V}$ staining buffer (35 mM Tris, $220 \mathrm{mM}$ glycine $\mathrm{pH} 8.3,14$ $\left.\mathrm{mM} \mathrm{MgSO}_{4}, 0.2 \%(\mathrm{w} / \mathrm{v}) \mathrm{Pb}\left(\mathrm{NO}_{3}\right)_{2}, 8 \mathrm{mM} \mathrm{ATP}\right)$ and incubated at $30^{\circ}$ until bands appeared. This method was modified from Wittig and Schägger, 2005.

\subsubsection{ATPase activity measurement with and without oligomycin}

ATPase activity of complex $\mathrm{V}$ was measured as the rate of ATP hydrolysis (Chen et al., 1956; Ackerman and Tzagoloff, 2007). In brief, mitochondria (0.2 $\mathrm{mg} / \mathrm{ml}$ ) were resuspended in assay buffer (50 mM Tris-sulfate, $\mathrm{pH}$ 8.5, $4 \mathrm{mM} \mathrm{MgSO}_{4}$ ) with or without $50 \mu \mathrm{M}$ oligomycin. The reaction was started by addition of $20 \mathrm{mM}$ ATP and stopped after $20 \mathrm{~min}$ at $37^{\circ} \mathrm{C}$ by adding $10 \%$ TCA. Reference samples were treated in the same manner, except that the TCA was added prior to ATP. After a clarifying spin (5 min, $16000 \mathrm{~g}$ ), the amount of inorganic phosphate in the supernatant was determined by an ammonium molybdate colorimetric assay. For this, an equal volume of ammonium molybdate reagent $(2 \%(\mathrm{w} / \mathrm{v})$ ascorbic acid, $0.5 \%(\mathrm{w} / \mathrm{v})$ ammonium molybdate, $1.2 \mathrm{~N}$ sulfuric acid) was added, the samples were incubated at $37^{\circ} \mathrm{C}$ for $2 \mathrm{~h}$, and the absorbance at $820 \mathrm{~nm}$ was monitored. The amount of released phosphate was determined based on a calibration curve with known phosphate concentrations.

\subsubsection{Fractionation of mitochondrial lysate through a sucrose cushion}

To pellet mitochondrial ribosomes and their associated proteins, $300 \mu \mathrm{g}$ of isolated mitochondria were lysed for $30 \mathrm{~min}$ on ice in lysis buffer containing $1 \%$ Triton X-100, 50 or $150 \mathrm{mM} \mathrm{NaCl}, 1 \mathrm{mM}$ PMSF, $20 \mathrm{mM} \mathrm{MgCl}_{2}$ and $20 \mathrm{mM}$ HEPES/KOH $\mathrm{pH}$ 7.4. Unsolubilized material was removed by a clarifying spin at $16000 \mathrm{~g}, 4^{\circ} \mathrm{C}$ for 10 minutes. Afterwards, one half of the lysate was directly precipitated with TCA, and the other half was underlayed with $50 \mu \mathrm{L}$ of a sucrose solution $(1.2 \mathrm{M}$ 
sucrose in $20 \mathrm{mM}$ Hepes/KOH pH 7.4) and centrifuged for $120 \mathrm{~min}$ at $145000 \mathrm{~g}$ at $4^{\circ} \mathrm{C}$. The supernatant was then collected and the pellet was resuspended in a lysis buffer. Proteins from all fractions were precipitated with $12 \%$ TCA and analyzed by SDS-PAGE and Western blotting.

\subsubsection{Chemical protein cross-linking in mitochondria}

For chemical cross-linking, mitochondria were thawed on ice and resuspended in $1 \mathrm{ml} / \mathrm{mg}$ of mitochondria cross-linking buffer (20 mM HEPES pH 7.2, $100 \mathrm{mM}$ $\mathrm{KCl}$ ). DSS or DSP, diluted in DMSO, were added to a final concentration of 250 $\mu \mathrm{M}$. Samples were incubated on ice for 30 minutes, then the reactions were quenched with $100 \mathrm{mM}$ Tris $/ \mathrm{HCl} \mathrm{pH} 7.4$ on ice for 10 minutes. Finally, mitochondria were pelleted by centrifugation at $16000 \mathrm{~g} 4^{\circ} \mathrm{C}$ for $10 \mathrm{~min}$, the pellet was resuspended in SDS-loading buffer, run on SDS-PAGE and analyzed by Western blotting.

\subsubsection{Protein assembly into mitochondrial protein complexes}

To perform assembly of radioactively labeled protein precursors into mitochondrial protein complexes, proteins were first imported into mitochondria and analyzed by BN-PAGE. In brief, $75 \mu \mathrm{g}$ of mitochondria per reaction were incubated in BSAimport buffer containing $0.4 \mathrm{M} \mathrm{KCl}, 40 \mathrm{mM}$ potassium phosphate buffer $\mathrm{pH}$ 7.4, 3 mM ATP, $3 \mathrm{mM}$ NADH, $9 \mathrm{mM}$ creatine phosphate and $10 \mu \mathrm{l}$ of $10 \mu \mathrm{g} / \mathrm{ml}$ creatine kinase. AVO-mix was added to the control samples to disrupt the membrane potential. $10 \mu \mathrm{l}$ of lysate were added per reaction and import/assembly was performed for 15 and $45 \mathrm{~min}$ time points. After this, $4 \mu \mathrm{l}$ of $1 \mathrm{mg} / \mathrm{ml}$ proteinase $\mathrm{K}$ was added to all samples followed by 15 min incubation on ice. Proteinase K was inhibited by addition of $2 \mathrm{mM}$ PMSF ( 5 minutes incubation on ice). Mitochondria were pelleted by centrifugation (10 min, $16000 \mathrm{~g}$ ), washed once with ice-cold SEM buffer, solubilized as described in 5.6.1 and analyzed by BN-PAGE and digital autoradiography.

\subsection{Miscellaneous}

Mitochondrial targeting signals were predicted using the MitoProt Server (Claros and Vincens, 1996). Protein transmembrane regions were predicted by TMpred (http : //www.ch.embnet.org/software/TMPRED form.html). 


\section{Bibliography}

Ackerman, S. H., Gatti, D. L., Gellefors, P., Douglas, M. G., and Tzagoloff, A. (1991). ATP13, a nuclear gene of Saccharomyces cerevisiae essential for the expression of subunit 9 of the mitochondrial ATPase. FEBS Lett., (278):234-238.

Ackerman, S. H. and Tzagoloff, A. (1990a). ATP10, a yeast nuclear gene required for the assembly of the mitochondrial F1-Fo complex. J. Biol. Chem., (265(17)):9952-9959.

Ackerman, S. H. and Tzagoloff, A. (1990b). Identification of two nuclear genes (ATP11, ATP12) required for assembly of the yeast F1-ATPase. Proc. Natl. Acad. Sci. USA, (87):4986-4990.

Ackerman, S. H. and Tzagoloff, A. (2002). Atp11p and Atp12p are chaperones for F(1)-ATPase biogenesis in mitochondria. Biochim. Biophys. Acta, (1555):101-105.

Ackerman, S. H. and Tzagoloff, A. (2007). Methods to determine the status of mitochondrial ATP synthase assembly. Methods Mol. Biol., (372):363-377.

Allen, J. F. (1993). Control of gene expression by redox potential and the requirement for chloroplast and mitochondrial genomes. J. Theor. Biol., (165):609-631.

Amiott, E. A. and Jaehning, J. A. (1983). Mitochondrial transcription is regulated via an ATP sensing mechanism that couples RNA abundance to respiration. Mol. Cell, (22):329-338.

Amma, I., Sommerb, T., and Wolfa, D. H. (2008). Protein quality control and elimination of protein waste: The role of the ubiquitin-proteasome system. BBA Mol. Biol. Res., (1843(1)):182-196.

Amunts, A., Brown, A., Bai, X. C., Liacer, J. L., Hussain, T., Emsley, P., Long, F., Murshudov, G., Scheres, S. H., and Ramakrishnan, V. (2014). Structure of the yeast mitochondrial large ribosomal subunit. Science, (343(6178)):1485-1489.

Anderson, S., Bankier, A. T., Barrell, B. G., de Bruijn, M. H., Coulson, A. R., and Drouin, J. (1981). Sequence and organization of the human mitochondrial genome. Nature, (290):457-465.

Andersson, S. G. E., Karlberg, O., Canbäck, B., and Kurland, C. G. (2003). On the origin of mitochondria: a genomics perspective. Philos. Trans. R. Soc. Lond. B. Biol. Sci., (358(1429)):165-177.

Andersson, S. G. E. and Kurland, C. G. (1998). Reductive evolution of resident genomes. Trends Microbiol., (6):263-268.

Andersson, S. G. E., Zomorodipour, A., Andersson, J. O., Sicheritz-Ponten, T., Alsmark, U. C., Podowski, R. M., Naslund, A. K., Eriksson, A. S., Winkler, H. H., and Kurland, C. G. (1998). The genome sequence of Rickettsia prowazekii and the origin of mitochondria. Nature, (396):133-140.

Arnold, I., Pfeiffer, K., Neupert, W., Stuart, R., and Shägger, H. (1998). Yeast mitochondrial F1Fo ATP synthase exists as a dimer: identification of three dimer-specific subunits. EMBO J., (17):7170-7178. 
Arroyo, J. D., Jourdain, A. A., Calvo, S. E., Ballarano, C. A., Doench, J. G., Root, D. E., and Mootha, V. K. (2016). A genome-wide CRISPR death screen identifies genes essential for oxidative phosphorylation. Cell Metab., (24(6)):875-885.

Atkinson, A., Smith, P., Fox, J. L., Cui, T. Z., Khalimonchuk, O., and Winge, D. R. (2011). The LYR protein Mzm1 functions in the insertion of the Rieske Fe/S protein in yeast mitochondria. Mol. Cell. Biol., (31):3988-3996.

Augustin, S., Nolden, M., Muller, S., Hardt, O., and I. Arnold, T. L. (2005). Characterization of peptides released from mitochondria: Evidence for constant proteolysis and peptide efflux. J. Biol. Chem., (280):2691-2699.

Baker, M. J., Tatsura, T., and Langer, T. (2011). Quality control of mitochondrial proteostasis. Cold Spring Harb. Perspect. Biol., (3):a007559.

Barrientos, A., Zambrano, A., and Tzagoloff, A. (2004). Mss51p and Cox14p jointly regulate mitochondrial Cox1p expression in Saccharomyces cerevisiae. EMBO J., (23):3472-3482.

Barros, M. H., Myers, A. M., Driesche, S. V., and Tzagoloff, A. (2006). COX24 codes for a mitochondrial protein required for processing of the COX1 transcript. J. Biol. Chem., (281):3743-3751.

Bauer, M., Behrens, M., Esser, K., Michaelis, G., and Pratje, E. (1994). PET102, a nuclear gene required for proteolytic processing of cytochrome oxidase subunit 2 in yeast. Mol. Gen. Genet., (245):272-278.

Bauerschmitt, H., Mick, D. U., Deckers, M., Vollmer, C., Funes, S., Kehrein, K., Ott, M., Rehling, P., and Herrmann, J. M. (2010). Ribosome-binding proteins Mdm38 and Mba1 display overlapping functions for regulation of mitochondrial translation. Mol. Biol. Cell., (21(12)):1937-1944.

Becker, T., Pfanner, S., Guiard, B., Stojanovski, D., Milenkovic, D., Kutik, S., Pfanner, N., Meisinger, C., and Wiedemann, N. (2008). Biogenesis of the mitochondrial TOM complex: Mim1 promotes insertion and assembly of signal-anchored receptors. J. Biol. Chem., (283):120-127.

Benz, R. (1994). Permeation of hydrophilic solutes through mitochondrial outer membranes: review on mitochondrial porins. Biochim. Biophys. Acta, (1197(2)):167-196.

Bonnefoy, N., Bsat, N., and Fox, T. D. (2001). Mitochondrial translation of Saccharomyces cerevisiae COX2 mRNA is controlled by the nucleotide sequence specifying the pre-Cox2p leader peptide. Mol. Cell. Biol., (21):2359-2372.

Bousquet, I., Dujardin, G., Poyton, R. O., and Slonimski, P. P. (1990). Two group I mitochondrial introns in the COB-box and COXI genes require the same MRS1/PET157 nuclear gene product for splicing. Curr. Genet., (18):117-124.

Boxma, B., de Graaf, R. M., van der Staay, G. W. M., van Alen, T. A., Ricard, G., Gabaldon, T., van Hoek, A. H. A. M., van der Staay, S. Y. M., Koopman, W. J. H., van Hellemond, J. J., Tielens, A. G. M., Friedrich, T., Veenhuis, M., Huynen, M. A., and Hackstein, J. H. P. (2005). An anaerobic mitochondrion that produces hydrogen. Nature, (434):74-79.

Brachmann, C. B., Davies, A., Cost, G. J., Caputo, E., Li, J., Hieter, P., and Boeke, J. D. (1998). Designer deletion strains derived from Saccharomyces cerevisiae S288C: a useful set of strains and plasmids for PCR-mediated gene disruption and other applications. Yeast, (14):115-132.

Braczynski, A. K., Vlaho, S., Müller, K., Wittig, I., Blank, A. E., Tews, D. S., Drott, U., Kleinle, S., Abicht, A., Horvath, R., Plate, K. H., Stenzel, W., Goebel, H. H., Schulze, A., Harter, P. N., Kieslich, M., and Mittelbronn, M. (2015). ATP synthase deficiency due to TMEM70 mutation leads to ultrastructural mitochondrial degeneration and is amenable to treatment. Biomed. Res. Int., page 462592. 
Brewer, L. R., Friddle, D., Noy, A., Baldwin, E., Martin, S. S., Corzett, M., Balhorn, R., and Baskin, R. J. (2003). Packaging of single DNA molecules by the yeast mitochondrial protein Abf2p. Biophys. J., (85):2519-2524.

Camougrand, N., Pelissier, P., Velours, G., and Guerin, M. (1995). NCA2, a second nuclear gene required for the control of mitochondrial synthesis of subunits 6 and 8 of ATP synthase in Saccharomyces cerevisiae. J. Mol. Biol., (247):588-596.

Capaldi, R. A. (1990). Structure and function of cytochrome c oxidase. Annu. Rev. Biochem., (59):569-595.

Caron, F., Jacq, C., and Rouviere-Yaniv, J. (1979). Characterization of a histone-like protein extracted from yeast mitochondria. Proc. Natl Acad. Sci. USA, (76):4265-4269.

Carr, H. S. and Winge, D. R. (2003). Assembly of cytochrome c oxidase within the mitochondrion. Acc. Chem. Res., (36):309-316.

Chacinska, A., Koehler, C. M., Milenkovic, D., Lithgow, T., and Pfanner, N. (2009). Importing mitochondrial proteins: machineries and mechanisms. Cell, (138(4)):628-644.

Chen, H., Detmer, S. A., Ewald, A. J., Griffin, E. E., Fraser, S. E., and Chan., D. C. (2003). Mitofusins Mfn1 and Mfn2 coordinately regulate mitochondrial fusion and are essential for embryonic development. J. Cell Biol., (160):189-200.

Chen, J. Y. and Martin, N. C. (1988). Biosynthesis of tRNA in yeast mitochondria. An endonuclease is responsible for the 3'-processing of tRNA precursors. J. Biol. Chem., (263):13677-13682.

Chen, P., Toribara, T., and Warner, H. (1956). Microdetermination of phosphorus. Anal. Chem., (28):17561758.

Christian, B. E. and Spremulli, L. L. (2010). Preferential selection of the 5'-terminal start codon on leaderless mRNAs by mammalian mitochondrial ribosomes. J. Biol. Chem., (285(36)):28379-28386.

Christianson, T. and Rabinowitz, M. (1983). Identification of multiple transcriptional initiation sites on the yeast mitochondrial genome by in vitro capping with guanylyltransferase. J. Biol. Chem., (258):1402514033.

Clapham, D. E. (2007). Calcium signaling. Cell, (1319(6)):1047-1058.

Claros, M. G. and Vincens, P. (1996). Computational method to predict mitochondrially imported proteins and their targeting sequences. Eur. J. Biochem., (241):779-786.

Cogliati, S., Calvo, E., Loureiro, M., Guaras, A. M., Nieto-Arellano, R., Garcia-Poyatos, C., Ezkurdia, I., Mercader, N., Vazquez, J., and Enriquez, J. A. (2016). Mechanism of super-assembly of respiratory complexes III and IV. Nature, (539(7630)):579-582.

Costanzo, M. C., Seaver, E. C., and Fox, T. D. (1989). The PET54 gene of Saccharomyces cerevisiae: characterization of a nuclear gene encoding a mitochondrial translational activator and subcellular localization of its product. Genetics, (122):297-305.

Cruciat, C. M., Hell, K., Fölsch, H., Neupert, W., and Stuart, R. A. (1999). Bcs1p, an AAA-family member, is a chaperone for the assembly of the cytochrome bc(1) complex. EMBO J., (18):5226-5233.

Dalrymple, G. B. (2001). The age of the Earth in the twentieth century: a problem (mostly) solved. Special Publications, Geological Society of London, (190(1)):205-221.

Davies, K. M., Anselmi, C., Wittig, I., Faraldo-Gómez, J. D., and Kühlbrandt, W. (2012). Structure of the yeast F1Fo-ATP synthase dimer and its role in shaping the mitochondrial cristae. (109(34)):1260212607. 
Davies, K. M., Daum, B., Gold, V. A., Mühleip, A. W., Brandt, T., Blum, T. B., Mills, D. J., and Kühlbrandt, W. (2014). Visualization of ATP synthase dimers in mitochondria by electron cryotomography. J. Vis. Exp., (91):51228.

de Vries, S. and Grievell, L. A. (1988). Purificationand characterization of a rotenone-insensitive NADH:Q6 oxidoreductase from mitochodria of S. cerevisiae. Eur. J. Biochem., (176):377-384.

Desai, N., Brown, A., Amunts, A., and Ramakrishnan, V. (2017). The structure of the yeast mitochondrial ribosome. Science, (355(6324)):528-531.

Devenish, R. J., Prescott, M., Roucou, X., and Nagley, P. (2000). Insights into ATP synthase assembly and function through the molecular genetic manipultion of subunits of the yeast mitochondrial enzyme complex. Biochim. Biophys. Acta, (1458):428-442.

Dieckmann, C. L., Koerner, T. J., and Tzagoloff, A. (1984). Assembly of the mitochondrial membrane system. CBP1, a yeast nuclear gene involved in 5' end processing of cytochrome $b$ pre-mRNA. J. Biol. Chem, (259):4722-4731.

Dieckmann, C. L., Pape, L. K., and Tzagoloff, A. (1982). Identification and cloning of a yeast nuclear gene (CBP1) involved in expression of mitochondrial cytochrome b. Proc. Natl. Acad. Sci. USA, (79(6)):18051809.

Dieckmann, C. L. and Tzagoloff, A. (1985). Assembly of the mitochondrial membrane system. CBP6, a yeast nuclear gene necessary for synthesis of cytochrome b. J. Biol. Chem, (260):1513-1520.

Diffley, F. and Stillman, B. (1991). A close relative of the nuclear, chromosomal high-mobility group protein HMG1 in yeast mitochondria. Proc. Natl. Acad. Sci. USA, (88):7864-7868.

Dimmer, K. S., Fritz, S., Fuchs, F., Messerschmitt, M., Weinbach, N., Neupert, W., and Westermann, B. (2002). Genetic basis of mitochondrial function and morphology in Saccharomyces cerevisiae. Mol. Biol. Cell., (13(3)):847-853.

Diodato, D., Invernizzi, F., Lamantea, E., Fagiolari, G., Parini, R., Menni, F., Parenti, G., Bollani, L., Pasquini, E., Donati, M. A., Cassandrini, D., Santorelli, F. M., Haack, T. B., Prokisch, H., Ghezzi, D., Lamperti, C., and Zeviani, M. (2015). Common and novel TMEM70 mutations in a cohort of italian patients with mitochondrial encephalocardiomyopathy. JIMD Rep., (15):71-78.

Dismukes, G. C., Klimov, V. V., Baranov, S. V., Kozlov, Y. N., DasGupta, J., and Tyryshkin, A. (2001). The origin of atmospheric oxygen on Earth: the innovation of oxygenic photosynthesis. Proc. Natl. Acad. Sci. USA, (98(5)):2170-2175.

Dontsova, O. A. and Dinman, J. D. (2005). 5s rRNA: structure and function from head to toe. Int. J. Biomed. Sci., (1):2-7.

Driessen, A. J. and Nouwen, N. (2008). Protein translocation across the bacterial cytoplasmic membrane. Annu. Rev. Biochem., (77):643-667.

Dunstan, H. M., Green-Willms, N. S., and Fox, T. D. (1997). In vivo analysis of Saccharomyces cerevisiae COX2 mRNA 5'-untranslated leader functions in mitochondrial translation initiation and translational activation. Genetics, (147):87-100.

Dziembowski, A., Piwowarski, J., Hoser, R., Minczuk, M., Dmochowska, A., Siep, M., van der Spek, H., Grivell, L., and Stepien, P. P. (2003). The yeast mitochondrial degradosome. Its composition, interplay between RNA helicase and RNase activities and the role in mitochondrial RNA metabolism. J. Biol. Chem., (278):1603-1611. 
Ellis, T. P., Helfenbein, K. G., Tzagoloff, A., and Dieckmann, C. L. (2004). Aep3p stabilizes the mitochondrial bicistronic mRNA encoding subunits 6 and 8 of the $\mathrm{H}+-$ translocating ATP synthase of Saccharomyces cerevisiae. J. Biol. Chem., (279):15728-15733.

Ellis, T. P., Lukins, H. B., Nagley, P., and Corner, B. E. (1999). Suppression of a nuclear aep2 mutation in Saccharomyces cerevisiae by a base substitution in the 5'-untranslated region of the mitochondrial OLI1 gene encoding subunit 9 of ATP synthase. Genetics, (151):1353-1363.

Embley, T. M., Horner, D. A., and Hirt, R. P. (1997). Anaerobic eukaryote evolution: hydrogenosomes as biochemically modified mitochondria? Trends Ecol. Evol., (12):437-441.

Finnegan, P. M., Ellis, T. P., Nagley, P., and Lukins, H. B. (1995). The mature AEP2 gene product of Saccharomyces cerevisiae, required for the expression of subunit 9 of ATP synthase, is a $58 \mathrm{kDa}$ mitochondrial protein. FEBS Lett., (368):505-508.

Finnegan, P. M., Payne, M. J., Kermidaris, E., and Lukins, H. B. (1991). Characterization of a yeast nuclear gene, AEP2, required for accumulation of mitochondrial mRNA encoding subunit 9 of the ATP synthase. Curr. Genet., (20):53-61.

Finsterer, J. and Kothari, S. (2014). Neonatal pulmonary hypertension in mitochondrial disorders due to TMEM70 mutations. Mol. Genet. Metab. Rep., (1):235-236.

Fischer, U., Rheinheimer, S., Krempler, A., Löbrich, M., and Meese, E. (2013). Glioma-amplified sequence KUB3 influences double-strand break repair after ionizing radiation. Int. J. Oncol., (43(1)):50-56.

Foury, F., Roganti, T., Lecrenier, N., and Purnelle, B. (1998). The complete sequence of the mitochondrial genome of Saccharomyces cerevisiae. FEBS Lett., (440):325-331.

Fox, T. D. (1979). Five TGA stop codons occur within the translated sequence of the yeast mitochondrial gene for cytochrome $c$ oxidase subunit II. PNAS, (76):6534-6538.

Francis, B. R. and Thorsness, P. E. (2011). Hsp90 and mitochondrial proteases Yme1 and Yta10/12 participate in ATP synthase assembly in Saccharomyces cerevisiae. Mitochondrion, (11):587-600.

Franklin, M. J., Brusilow, W. S. A., and Woodbury, D. J. (2004). Determination of proton flux and conductance at pH 6.8 through single Fo sector from Escherichia coli. Biophys. J., (87):3594-3599.

Frazier, A. E., Taylor, R. D., Mick, D. U., Warscheid, B., Stoepel, N., Meyer, H. E., Ryan, M. T., Guiard, B., and Rehling, P. (2006). Mdm38 interacts with ribosomes and is a component of the mitochondrial protein export machinery. J. Cell. Biol., (172(4)):553-564.

Fujikawa, M., Sugawara, K., Tanabe, T., and Yoshida, M. (2015). Assembly of human mitochondrial ATP synthase through two separate intermediates, F1-c-ring and b-e-g complex. FEBS Lett., (589):27072712 .

Gagliardi, D., Stepien, P. P., Temperley, R. J., Lightowlers, R. N., and Chrzanowska-Lightowlers, Z. M. (2004). Messenger RNA stability in mitochondria: different means to an end. Trends Genet., (20):260267.

Gampel, A., Nishikimi, M., and Tzagoloff, A. (1989). Cbp2 protein promotes in vitro excision of a yeast mitochondrial group I intron. Mol. Biol. Cell, (9):5424-5433.

Garofalo, C., Trinko, R., Kramer, G., Appling, D. R., and Hardesty, B. (2003). Purification and characterization of yeast mitochondrial initiation factor 2. Arch. Biochem. Biophys., (413):243-252. 
Gebert, N., Gebert, M., Oeljeklaus, S., von der Malsburg, K., Stroud, D. A., Kulawiak, B., C. Wirth, a. R. P. Z., Dolezal, P., Wiese, S., Simon, O., Schulze-Specking, A., Truscott, K. N., Sickmann, A., Rehling, P., Guiard, B., Hunte, C., Warscheid, B., van der Laan, M., Pfanner, N., and Wiedemann, N. (2011). Dual function of Sdh3 in the respiratory chain and TIM22 Protein Translocase of the mitochondrial inner membrane. Molecular Cell, (44(5)):811-818.

Genova, M. L. and Lenaz, G. (2011). New development on the functions of coenzyme Q in mitochondria. BioFactors (Oxford, England), (37(5)):330-353.

Gietz, R. D. and Schiestl, R. H. (2007). Quick and easy yeast transformation using the LiAc/SScarrier DNA/PEG method. Nature Protocols, (2(1)):35-37.

Gilbert, L. A., Horlbeck, M. A., Adamson, B., Villalta, J. E., Chen, Y., Whitehead, E. H., Guimaraes, C., Panning, B., Ploegh, H. L., Bassik, M. C., Qi, L. S., Kampmann, M., and Weissman, J. S. (2014). Genome-scale CRISPR-mediated control of gene repression and activation. Cell, (159(3)):647-661.

Goyon, V., Fronzes, R., Salin, B., di Rago, J.-P., Velours, J., and Brethes, D. (2008). Yeast cells depleted in Atp14p fail to assemble Atp6p within the ATP synthase and exhibit altered mitochondrial cristae morphology. J. Biol. Chem., (283):9749-9758.

Gray, M. W., Burger, G., and Lang, B. F. (1999). Mitochondrial evolution. Science, (283):1476-1481.

Gray, M. W. and Lang, B. F. (1998). Transcription in chloroplasts and mitochondria: a tale of two polymerases. Trends Microbiol., (6):1-3.

Green, D. R. and Reed, J. C. (1998). Mitochondria and apoptosis. Science, (281(5381)):1309-1312.

Green-Willms, N. S., Butler, C. A., Dunstan, H. M., and Fox, T. D. (2001). Pet111p, an inner membranebound translational activator that limits expression of the Saccharomyces cerevisiae mitochondrial gene COX2. J. Biol. Chem., (276):6392-6397.

Greenleaf, A. L., Kelly, J. L., and Lehman, I. R. (1986). Yeast RPO41 gene product is required for transcription and maintenance of the mitochondrial genome. Proc. Natl. Acad. Sci. USA, (83):33913394.

Gruschke, S., Kehrein, K., Römpler, K., Gröne, K., Israel, L., Imhof, A., Herrmann, J. M., and Ott, M. (2011). Cbp3-Cbp6 interacts with the yeast mitochondrial ribosomal tunnel exit and promotes cytochrome $b$ synthesis and assembly. J. Cell. Biol., (193(6)):1101-1114.

Gruschke, S., Römpler, K., Hildenbeutel, M., Kehrein, K., Kühl, I., Bonnefoy, N., and Ott, M. (2012). The Cbp3-Cbp6 complex coordinates cytochrome $b$ synthesis with bc(1) complex assembly in yeast mitochondria. J. Cell. Biol., (199(1)):137-150.

Gueldener, U., Heinisch, J., Koehler, G. J., Voss, D., and Hegemann, J. H. (2002). A second set of loxP marker cassettes for Cre-mediated multiple gene knockouts in budding yeast. Nucleic Acids Research, (30(6)):e23.

Guenebaut, V., Vincentelli, R., Mills, D., Weiss, H., and Leonard, K. R. (1997). Three-dimensional structure of NADH-dehydrogenase from Neurospora crassa by electron microscopy and conical tilt reconstruction. J. Mol. Biol., (265):409-418.

Hanahan, D. (1983). Studies on transformation of Escherichia coli with plasmids. J. Mol. Biol., (166(4)):557-580.

Hatefi, Y. (1985). The mitochondrial electron transport and oxidative phosphorylation system. Annu. Rev. Biochem., (54):1015-1069. 
Heinemeyer, J., Braun, H. P., Boekeman, E. J., and Kouril, R. (2007). A structural model of the cytochrome $c$ reductase/oxidase supercomplex from yeast mitochondria. J Biol Chem., (282):12240-12248.

Helfenbein, K. G., Ellis, T. P., Dieckmann, C. L., and Tzagoloff, A. (2003). ATP22, a nuclear gene required for expression of the Fo sector of mitochondrial ATPase in Saccharomyces cerevisiae. J. Biol. Chem., (278):19751-19756.

Hell, K., Neupert, W., and Stuart, R. A. (2001). Oxa1p acts as a general membrane insertion machinery for proteins encoded by mitochondrial DNA. EMBO J., (20):1281-1288.

Hildenbeutel, M., Hegg, E. L., Stephan, K., Gruschke, S., Meunier, B., and Ott, M. (2014). Assembly factors monitor sequential hemylation of cytochrome $b$ to regulate mitochondrial translation. J. Cell. Biol., (2054):511-524.

Huang, H. R., Rowe, C. E., Mohr, S., Jiang, Y., Lambowitz, A. M., and Perlman, P. S. (2004). The splicing of yeast mitochondrial group I and group II introns requires a DEAD-box protein with RNA chaperone function. PNAS, (102(1)):163-168.

Hunte, C., Koepke, J., Lange, C., Rossmanith, T., and Michel, H. (2000). Structure at 2.3 A resolution of the cytochrome bc(1) complex from the yeast Saccharomyces cerevisiae co-crystallized with an antibody Fv fragment. Structure, (8):669-684.

Ishihara, N., Fujita, Y., Oka, T., and Mihara., K. (2006). Regulation of mitochondrial morphology through proteolytic cleavage of OPA1. EMBO J., (25):2966-2977.

Islas-Osuna, M. A., Ellis, T. P., Marnell, L. L., Mittelmeier, T. M., and Dieckmann, C. L. (2002). Cbp1 is required for translation of the mitochondrial cytochrome $b$ mRNA of Saccharomyces cerevisiae. Proc Natl Acad Sci USA, (277(41)):37987-37990.

Jacobs, H. T. (2001). Making mitochondrial mutants. Trends in Genetics, (17(11)):653-660.

Jang, S. H. and Jaehning, J. A. (1991). The yeast mitochondrial RNA polymerase specificity factor, MTF1, is similar to bacterial sigma factors. J. Biol. Chem., (266):22671-22677.

Janke, C., Magiera, M. M., Rathfelder, N., Taxis, C., Reber, S., Maekawa, H., Moreno-Borchart, A., Doenges, G., Schwob, E., Schiebel, E., and Knop, M. (2004). A versatile toolbox for PCR-based tagging of yeast genes: new fluorescent proteins, more markers and promoter substitution cassettes. Yeast, (21):947-962.

Jia, L., Dienhart, M. K., Schramp, M., McCauley, M., Hell, K., and Stuart, R. (2003). Yeast Oxa1 interacts with mitochondrial ribosomes: the importance of the C-terminal hydrophilic region of Oxa1. EMBO J., (22):6438-6447.

Jia, L., Dienhart, M. K., and Stuart, R. A. (2007). Oxa1 directly interacts with Atp9 and mediates its assembly into the mitochondrial F1Fo-ATP synthase complex. Mol. Biol. Cell, (18):1897-1908.

Kalinovich, A. V., de Jong, J. M., Cannon, B., and Nedegaard, J. (2017). UCP1 in adipose tissues: two steps to full browning. Biochimie, (134):127-137.

Karnkowska, A., Vacek, V., Zubakova, Z., Treitli, S. C., Petrzelkova, R., Eme, L., Novak, L., Zarsky, V., Barlow, L. D., Herman, E. K., Soukal, P., Hroudova, M., Dolezal, P., Stairs, C. W., Roger, A. J., Elias, M., Dacks, J. B., Vicek, C., and Hampl, V. (2016). A eukaryote without a mitochondrial organelle. Curr. Biol., (23(10)):1274-1284.

Kasting, J. F. (1993). Earth's early atmosphere. Science, (259):920-926. 
Kehrein, K., Schilling, R., Möller-Hergt, B. V., Wurm, C. A., Jakobs, S., Lamkemeyer, T., Langer, T., and Ott, M. (2015). Organization of mitochondrial gene expression in two distinct ribosome-containing assemblies. Cell Reports, (10):843-853.

Kemper, C., Habib, S. J., Engl, G., Heckmeyer, P., Dimmer, K. S., and Rapaport, D. (2008). Integration of tail-anchored proteins into the mitochondrial outer membrane does not require any known import components. J. Cell. Sci., (121):1990-1998.

Koehler, C. M., Merchant, S., Oppliger, W., Schmid, K., Jarosch, E., Dolfini, L., Junne, T., Schatz, G., and Tokatkidis, K. (1998). Tim9p, an essential partner subunit of Tim10p for the import of mitochondrial carrier proteins. EMBO J., (17):6477-6486.

Kohler, R., Boehringer, D., Greber, B., Bingel-Erlenmeyer, R., Collinson, I., Schaffitzel, C., and Ban, N. (2009). YidC and Oxa1 form dimeric insertion pores on the translating ribosome. Mol. Cell, (34):344353.

Kozak, L. P., Britton, J. H., Kozak, U. C., and Wells, J. M. (1988). The mitochondrial uncoupling protein gene. Correlation of exon structure to transmembrane domains. J. Biol. Chem., (263(25)):12274-12277.

Krause, K., de Souza, R. L., Roberts, D. G., and Dieckmann, C. L. (2004). The mitochondrial messagespecific mRNA protectors Cbp1 and Pet309 are associated in a high-molecular weight complex. Mol. Biol. Cell., (15):2674-2683.

Kulak, N. A., Pichler, G., Paron, I., Nagarai, N., and Mann, M. (2014). Minimal, encapsulated proteomicsample processing applied to copy-number estimation in eukaryotic cells. Nat. Methods, (11):319-324.

Kumazaki, K., Chiba, S., Takemoto, M., Furukawa, A., Nishiyama, K., Sugano, Y., Mori, T., Dohmae, N., Hirata, K., Nakada-Nakura, Y., Maturana, A. D., Tanaka, Y., Mori, H., Sugita, Y., Arisaka, F., Ito, K., Ishitani, R., Tsukazaki, T., and Nureki, O. (2014a). Structural basis of Sec-independent membrane protein insertion by YidC. Nature, (509(7501)):516-520.

Kumazaki, K., Kishimoto, T., Furukawa, A., Mori, H., Tanaka, Y., Dohmae, N., Ishitani, R., Tsukazaki, T., and Nureki, O. (2014b). Crystal structure of Escherichia coli YidC, a membrane protein chaperone and insertase. Sci. Rep., (4:7299):doi: 10.1038/srep07299.

Kump, L. R. (2008). The rise of atmospheric oxygen. Nature, (451(7176)):277-278.

Kurland, C. G. and Andersson, S. G. E. (2000). Origin and evolution of the mitochondrial proteome. Microbiol. Mol. Biol. Revl., (64):786-820.

Laemmli, U. (1970). Cleavage of structural proteins during the assembly of the head of bacteriophage T4. Nature, (227(5259)):680-685.

Lang, B. F., Gray, M. W., and Burger, G. (1999). Mitochondrial genome evolutionand the origin of eukaryotes. Annu. Rev. Genet., (33):351-397.

Lefebvre-Legendre, L. (2001). Identification of nuclear gene (FMC1) required for the asembly/stability of yeast mitochondrial F1-ATPase in heat stress conditions. J Biol Chem., (276):67891-67896.

Lenaz, G., Baracca, A., Barbero, G., Bergamini, C., Dalmonte, M. E., Sole, M. D., Faccioli, M., Falasca, A., Fato, R., Genova, M. L., Sgarbi, G., and Solaini, G. (2010). Mitochondrial respiratory chain supercomplex I-III in physiology and pathology. Biochim. Biophys. Acta, (1797(6-7)):633-640.

Lenaz, G. and Genova, M. L. (2010). Structure and understanding of mitochondrial respiratory complexes: a new understanding of an old subject. Antioxidants and Redox Signalling, 12(8):961-1008. 
Letts, J. A., Fiedorczuk, K., and Sazanov, L. A. (2016). The architecture of respiratory supercomplexes. Nature, (537(7622)):644-648.

Liberek, K., Lewandowska, A., and Zietkiewicz, S. (2008). Chaperones in control of protein disaggregation. EMBO J., (27(2)):328-335.

Lill, R. and Mühlenhoff, U. (2008). Maturation of iron-sulfur proteins in eukaryotes: mechanisms, connected processes, and diseases. Annu. Rev. Biochem., (77):669-700.

Lipinski, K. A., Kaniak-Golik, A., and Golik, P. (2010). Maintenance and expression of the S. cerevisiae mitochondrial genome - From genetics to evolution and systems biology. BBA, (1797):1086-1098.

Ludlam, A. (2009). Chaperones of F1-ATPase. J. Biol. Chem., (284):17138-17146.

Lytovchenko, O., Naumenko, N., Oeljeklaus, S., Schmidt, B., von der Malsburg, K., Warscheid, B., van der Laan, M., and Rehling, P. (2014). The INA complex facilitates assembly of the peripheral stalk of the mitochondrial F1Fo-ATP synthase. EMBO J., (33):1624-1638.

Mai, Z., Ghosh, S., Frisardi, M., Rosenthal, B., Rogers, R., and Samuelson, J. (1999). Hsp60 is targeted to a cryptic mitochondrion-derived organelle ("crypton") in microaerophilic protozoan parasite Entamoeba histolytica. Mol. Cell. Biol., (19):2198-2205.

Manthey, G. M. and McEwen, J. E. (1995). The product of the nuclear gene PET309 is required for translation of mature mRNA and stability or production of intron-containing RNAs derived from the mitochondrial COX1 locus of Saccharomyces cerevisiae. EMBO J., (14):4031-4043.

Martin, W. and Hermann, R. G. (1998). Gene transfer from organelles to the nucleus: how much, what happens, and why? Plant Physiol., (118):9-17.

Martin, W., Hoffmeister, M., Rotte, C., and Henze, K. (2001). An overview of endosymbiotic models for the origins of eukaryotes, their ATP-producing organelles (mitochondria and hydrogenosomes), and their heterotrophic lifestyle. Biol. Chem., (382):1521-1539.

Mayorga, J. P., Camacho-Villasana, Y., Shingu-Vazquez, M., Garcia-Villegas, R., A. Zamudio-Ochoa, a. E. G.-G., Hernandez, G., and Perez-Martinez, X. (2016). A novel function of Pet54 in regulation of Cox1 synthesis in Saccharomyces cerevisiae mitochondria. J. Biol. Chem., (291(17)):9343-9355.

Meisinger, C., Pfanner, N., and Truscott, K. (2006). Isolation of yeast mitochondria. Methods Mol Biol, (313):33-39.

Melin, J., Kilisch, M., Neumann, P., Lytovchenko, O., Gomkale, R., Schendzielorz, A., Schmidt, B., Liepold, T., Ficner, R., Jahn, O., Rehling, P., and Schulz, C. (2015). A presequence-binding groove in Tom70 supports import of Mdl1 into mitochondria. Biochim. Biophys. Acta, (1853(8)):1850-1859.

Mentel, M. and Martin, W. (2008). Energy metabolism among eukaryotic anaerobes in light of Proterozoic ocean chemistry. Phil. Trans. R. Soc. L. B: Biol. Sci., (363):2717-2729.

Mesecke, N., Terziyska, N., Kozany, C., Baumann, F., Neupert, W., Hell, K., and Herrmann, J. M. (2005). A disulfide relay system in the intermembrane space of mitochondria that mediates protein import. Cell, (121):1059-1069.

Michon, T., Galante, M., and Velours, J. (1988). NH2-terminal sequence of the isolated yeast ATP synthase subunit 6 reveals post-translational cleavage. Eur. J. Biochem., (172):621-625.

Mick, D. U., Fox, T. D., and Rehling, P. (2011). Inventory control: cytochrome $c$ oxidase assembly regulates mitochondrial translation. Nat. Rev. Mol. Cell. Biol., (12):14-20. 
Mick, D. U., Vukotic, M., Piechura, H., Meyer, H. E., Warscheid, B., Deckers, M., and Rehling, P. (2010). Coa3 and Cox14 are essential for negative feedback regulation of COX1 translation in mitochondria. J. Cell. Biol., (191):142-154.

Mileykovskaya, E., Penczek, P. A., Fang, J., Mallampalli, V. K., Sparagna, G. C., and Dowhan, W. (2012). Arrangement of the respiratory chain complexes in Saccharomyces cerevisiae supercomplex III2IV2 revealed by single particle cryo-electron microscopy. J. Biol. Chem., (287(27)):23095-23103.

Miller, S. L. (1953). A production of amino acids under possible primitive earth conditions. Science, $(117(3046)): 528-529$.

Mishra, P. and Chan, D. C. (2017). Mitochondrial dynamics and inheritance during cell division, development and disease. Nat. Rev. Mol. Cell. Biol., (10):634-646.

Miyakawa, I., Sando, N., Kawano, K., Nakamura, S., and Kuroiwa, T. (1987). Isolation of morphologically intact mitochondrial nucleoids from the yeast, Saccharomyces cerevisiae. J. Cell Sci., (88):431-439.

Müller, M. (1993). The hydrogenosome. J. Gen. Microbiol, (139):2879-2889.

Müller, P. P. and Fox, T. D. (1984). Molecular cloning and genetic mapping of the PET494 gene of Saccharomyces cerevisiae. Mol. Gen. Genet., (195(1-2)):275-280.

Nagamori, S., Smirnova, I. N., and Kaback, H. R. (2004). Role of YidC in folding of polytopic membrane proteins. J. Cell. Biol., (165):53-62.

Naithani, S., Saracco, S. A., Butler, C. A., and Fox, T. D. (2003). Interactions among COX1, COX2, and COX3 mRNA-specific translational activator proteins on the inner surface of the mitochondrial inner membrane of Saccharomyces cerevisiae. Mol. Biol. Cell., (14(1)):324-333.

Nakamoto, R. K., Scanlon, J. A. B., and Al-Shawi, M. K. (2008). The rotatory mechanism of ATP synthase. Arch. Biochem. Biophys., (1553):188-211.

Newman, S. M., Zelenaya-Troitskaya, O., Perlman, P. S., and Butow, R. A. (1996). Analysis of mitochondrial DNA nucleoids in wild-type and a mutant strain of Saccharomyces cerevisiae that lacks the mitochondrial HMG-box protein, Abf2p. Nucleic Acids Res., (24):386-393.

Nicholls, D. G., Bernson, V. S., and Heaton, G. M. (1978). The identification of the component in the inner membrane of brown adipose tissue mitochondria responsible for regulating energy dissipation. Experientia. Supplementum., (32):89-93.

Noji, H., Zasuda, R., Yoshida, M., and Kinosita, K. (1997). Direct observation of the rotation of F1 ATP synthase. Nature, (386):299-302.

Olson, J. M. (2006). Photosynthesis in the Archean era. Photosyn. Res, (88(2)):109-117.

Osinga, K. A., Haan, M. D., Christianson, T., and Tabak, H. F. (1982). A nonanucleotide sequence involved in promotion of ribosomal RNA synthesis and RNA priming of DNA replication in yeast mitochondria. Nucleic Acids Res., (10):7993-8006.

Osinga, K. A., Vries, E. D., der Horst, G. T. V., and Tabak, H. F. (1984). Processing of yeast mitochondrial messenger RNAs at a conserved dodecamer sequence. EMBO J., (3):829-834.

Osman, C., Wilmes, C., Takashi, T., and Langer, T. (2007). Prohibitins interact genetically with Atp23, a novel processing peptidase and chaperone for the F1Fo -ATP synthase. Mol. Biol. Cell, (18):627-635.

Ott, M. and Herrmann, J. M. (2010). Co-translational membrane insertion of mitochondrially encoded proteins. Biochim. Biophys. Acta., (1803):767-775. 
Ott, M., Prestele, M., Bauerschmitt, H., Funes, S., N., and Herrmann, J. M. (2006). Mba1, a membraneassociated ribosome receptor in mitochondria. EMBO J., (25):1603-1610.

Palade, G. E. (1964). The organisation of living matter. Proc. Natl. Acad. Sci. USA, (52(2)):613-634.

Papic, D., Krumpe, K., Dukanovic, J., Dimmer, K. S., and Rapaport, D. (2011). Multispan mitochondrial outer membrane protein Ugo1 follows a unique Mim1-dependent import pathway. J. Cell. Biol., (194):397-405.

Paumard, P., Vaillier, J., Coulary, B., Schaeer, J., Soubannier, V., Mueller, D. M., and Brethes, D. (2002). The ATP synthase is involved in generating mitochondrial cristae morphology. EMBO J., (21(3)):221230 .

Payne, M. J., Finnegan, P. M., Smooker, P. M., and Lukins, H. B. (1993). Characterization of a second nuclear gene, AEP1, required for expression of the mitochondrial OLI1 gene in Saccharomyces cerevisiae. Curr. Genet, (24):126-135.

Pel, H. J. and Grivell, L. A. (1993). The biology of yeast mitochondrial introns. Mol. Biol. Rep., (18):1-13.

Pel, H. J., Maat, C., Rep, M., and Grivell, L. A. (1992). The yeast nuclear gene MRF1 encodes a mitochondrial peptide chain release factor and cures several mitochondrial RNA splicing defects. Nucleic Acids Res, (20):6339-6346.

Pelissier, P., Camougrand, N., Velours, G., and Guerin, M. (1995). NCA3, a nuclear gene involved in the mitochondrial expression of subunits 6 and 8 of the F1-Fo ATP synthase of S. cerevisiae. Curr. Genet., (27):409-416.

Perez-Martinez, X., Broadley, S. A., and Fox, T. D. (2003). Mss51p promotes mitochondrial Cox1p synthesis and interacts with newly synthesized Cox1p. EMBO J., (22):5951-5961.

Pfeffer, S., Woellhaf, M. W., Herrmann, J. M., and Forster, F. (2015). Organization of the mitochondrial translation machinery studied in situ by cryoelectron tomography. Nat. Commun., (6:6019).

Planavsky, N. J., Asael, D., Hofmann, A., Reinhard, C. T., Lalonde, S. V., Knudsen, A., Wang, X., Ossa, F. O., Pecoits, E., Smith, A. J. B., Beukes, N. J., Bekker, A., Johnson, T. M., Konhauser, K. O., Lyons, T. W., and Rouxel, O. J. (2014). Evidence for oxygenic photosynthesis half a billion years before the great oxidation event. Nature Geoscience, (7):283-286.

Poynton, R. O., Goehring, B., Droste, M., Sevarino, K. A., Allen, L. A., and Yhao, X. J. (1995). Cytochrome c oxidase from Saccharomyces cerevisiae. Methods Enzymol., (260):97-116.

Preuss, M., Leonhard, K., Hell, K., Stuart, R. A., Neupert, W., and Herrmann, J. M. (2001). Mba1, a novel component of the mitochondrial protein export machinery of the yeast Saccharomyces cerevisiae. J. Cell. Biol., (153):1085-1096.

Rak, M., Gokova, S., and Tzagoloff, A. (2011). Modular assembly of yeast mitochondrial ATP synthase. EMBO J., (30(5)):920-930.

Rak, M., Su, C. H., Xu, J. T., Azripoz, R., Singh, A. M., and Tzagoloff, A. (2016). Regulation of mitochondrial translation of the ATP8/ATP6 mRNA by Smt1p. Mol. Biol. Cell, (27):919-929.

Rak, M. and Tzagoloff, A. (2009). F1-dependent translation of mitochondrially encoded Atp6p and Atp8p subunits of yeast ATP synthase. Proc. Natl. Acad. Sci. USA, (106):18509-18514.

Rehling, P., Brandner, K., and Pfanner, N. (2004). Mitochondrial import and the twin-pore translocase. Nat. Rev. Mol. Cell Biol, (5):519-530. 
Rehling, P., Model, K., Brandner, K., Kovermann, P., Sickmann, A., Meyer, H. E., Kühlbrandt, W., Wagner, R., Truscott, K. N., and Pfanner, N. (2003). Protein insertion into the mitochondrial inner membrane by a twin-pore translocase. Science, (299):1747-1751.

Richter-Dennerlein, R., Dennerlein, S., and Rehling, P. (2015). Integrating mitochondrial translation into the cellular context. Nat. Rev. Mol. Cell. Biol., (16(10)):586-592.

Rieger, K. J., Aljinovic, G., Lazowska, J., Pohl, T. M., and Slonimski, P. P. (1997). A novel nuclear gene, $C B T 1$, essential for mitochondrial cytochrome $b$ formation: terminal processing of mRNA and intron-dependence. Curr. Genet., (32):163-174.

Rödel, G. (1986). Two yeast nuclear genes, $C B S 1$ and CBS2, are required for translation of mitochondrial transcripts bearing the 5'-untranslated COB leader. Curr. Genet, (11(1)):41-45.

Rödel, G. and Fox, T. D. (1987). The yeast mitochondrial CBS1 gene is required for translation of mitochondrial mRNAs bearing the COB 5'-untranslated leader. Mol. Gen. Genet., (206):45-50.

Rödel, G., Körte, A., and Kaudewitz, F. (1985). Mitochondrial suppression of a yeast nuclear mutation which affects the translation of the mitochondrial apocytochrome $b$ transcript. Curr. Genet, (9):641-648.

Roloff, G. A. and Henry, M. F. (2015). Mam33 promotes cytochrome c oxidase subunit I translation in Saccharomyces cerevisiae mitochondria. Mol. Biol. Cell., (26(16)):2885-2894.

Ruan, L., Zhou, C., Jin, E., Kucharavy, A., Zhang, Y., Wen, Z., Florens, L., and Li, R. (2017). Cytosolic proteostasis through importing of misfolded proteins into mitochondria. Nature, (543(7645)):443-446.

Rühle, T. and Leister, D. (2015). Assembly of F1Fo ATP synthases. BBA, (1847):849-860.

Ryan, M. T., Wagner, R., and Pfanner, N. (2000). The transport machinery for the import of preproteins across the outer mitochondrial membrane. Int. J. Biochem. Cell Biol., (32):13-21.

Sambrook, J. J. and Russell, D. D. W. (2001). Molecular cloning. A laboratory manual. A laboratory manual. CSHL Press, (3rd edn.).

Sanchirico, M. (1998). Understanding mitochondrial biogenesis through gene relocation. PhD thesis, University of Massachussetts, Amherst, MA.

Saracco, S. A. and Fox, T. D. (2002). Cox18p is required for export of the mitochondrially encoded Saccharomyces cerevisiae Cox2p C-tail and interacts with Pnt1p and Mss2p in the inner membrane. Mol. Biol. Cell, (13):1122-1131.

Schägger, H. (2001). Respiratory chain supercomplexes. IUBMB Life, (52):119-128.

Schägger, H. and Jagow, G. (1991). Blue native electrophoresis for isolation of membrane protein complexes in enzymatically active form. Anal. Biochem., (199):223-231.

Schatz, G. (1968). Impaired binding of mitochondrial adenosine triphosphatase in the cytoplasmic petite mutant of Saccharomyces cerevisiae. J. Biol. Chem., (243):2192-2199.

Schatz, G. and Dobberstein, B. (1996). Common principles of protein translocation across membranes. Science, (271):1519-1526.

Schmidt, O., Pfanner, N., and Meisinger, C. (2010). Mitochondrial protein import: from proteomics to functional mechanisms. Nat. Rev. Mol. Cell Biol., (11):655-667.

Schneider, A. (2011). Mitochondrial tRNA import and its consequences for mitochondrial translation. Annu. Rev. Biochem, (80):1033-1053. 
Schulz, C., Schendzielorz, A., and Rehling, P. (2015). Unlocking the presequence import pathway. Trends Cell Biol., (25):265-275.

Senior, A. E., Nadanaciva, S., and Weber, J. (2002). The molecular mechanism of ATP synthesis by F1Fo ATP synthase. BBA, (1553):188-211.

Seytter, T., Lottspeich, F., Neupert, W., and Schwarz, E. (1998). Mam33p, an oligomeric, acidic protein in the mitochondrial matrix of Saccharomyces cerevisiae is related to the human complement receptor gC1q-R. Yeast, (14(4)):303-310.

Shadel, G. S. and Horvath, T. L. (2015). Mitochondrial ROS signaling in organismal homeostasis. Cell, (163(3)):560-569.

Shiiba, D., Fumoto, S. I., Miyakawa, I., and Sando, N. (1997). Isolation of giant mitochondrial nucleoids from the yeast Saccharomyces cerevisiae. Protoplasma, (198):177-185.

Shiota, T., Imai, K., Qiu, J., Hewitt, V. L., Tan, K., Shen, H.-H., Sakiyama, N., Fukasawa, Y., Hayat, S., Kamiya, M., and et al. (2015). Molecular architecture of the active mitochondrial protein gate. Science, (349):1544-1548.

Shutt, T. E. and Gray, M. W. (2006). Bacteriophage origins of mitochondrial replication and transcription proteins. Trends Genet., (22):90-95.

Sickmann, A. (2003). The proteome of Saccharomyces cerevisiae mitochondria. PNAS, (100):13207-13212.

Sideris, D. P., Petis, N., Katrakili, N., Mikropoulou, D., Gallo, A., Ciofi-Baffoni, S., Banci, L., Bertini, I., and Tokatlidis, K. (2009). A novel intermembrane space-targeting signal docks cysteines onto Mia40 during mitochondrial oxidative folding. J. Cell. Biol., (187):1007-1022.

Song, J., Tamura, Y., Yoshihisa, T., and Endo, T. (2014). A novel import route for an N-anchor mitochondrial outer membrane protein aided by the TIM23 complex. EMBO Rep., (15):670-677.

Souza, R. L., Green-Willms, N. S., Fox, T. D., Tzagoloff, A., and Nobrega, F. G. (2000). Cloning and characterization of COX18, a Saccharomyces cerevisiae PET gene required for the assembly of cytochrome c oxidase. J. Biol. Chem., (275):14898-14902.

Stock, D., Leslie, A. G. W., and Walker, J. E. (1999). Molecular architechture of the rotary motor in ATP synthase. Science, (286):1700-1705.

Strauss, M., Hofhaus, G., Schröder, R. R., and Kühlbrandt, W. (2008). Dimer ribbons of ATP synthase shape the inner mitochondrial membrane. EMBO J., (27):1154-1160.

Stribinskis, J. V., Gao, G. J., Sulo, P., Dang, Y. L., and Martin, N. C. (1996). Yeast mitochondrial RNase $\mathrm{P}$ RNA synthesis is altered in an RNase $\mathrm{P}$ protein subunit mutant: insights into the biogenesis of a mitochondrial RNA-processing enzyme. Mol. Cell. Biol., (16):3429-3436.

Sugiura, A., Mattie, S., Prudent, J., and McBride, H. M. (2017). Newly born peroxisomes are a hybrid of mitochondrial and ER-derived pre-peroxisomes. Nature, (542(7640)):251-254.

Symersky, J., Pagadala, V., Osowski, D., Krah, A., Meier, T., Faraldo-Gomez, J. D., and Mueller, D. M. (2012). Structure of the c(10) ring of the yeast mitochondrial ATP synthase in the open conformation. Nat. Struct. Mol. Biol., (19(5)):485-491.

Taguchi, N., Ishihara, N., Jofuku, A., Oka, T., and Mihara., K. (2007). Mitotic phosphorylation of dynamin-related GTPase Drp1 participates in mitochondrial fission. J. Biol. Chem., (282(15)):1152111529 . 
Tovar, J., Fisher, A., and Clark, C. G. (1999). The mitosome, a novel organelle related to mitochondria in a mitochondrial parasite Entamoeba histolytica. Mol. Microbiol., (32):1013-1021.

Tovar, J., Leon-Avila, G., Sanchez, L. B., and Sutak, R. (2003). Mitochondrial remnant organelles of Giardia function in iron-sulphur protein maturation. Nature, (426):127-128.

Towpik, J. (2005). Regulation of mitochondrial translation in yeast. Cell. Mol. Biol. Lett., (10):571-594.

Truscott, K. N., Kovermann, P., Geissler, A., Merlin, A., Meijer, M., Driessen, A. J., Rassow, J., Pfanner, N., and Wagner, R. (2001). A presequence- and voltage-sensitive channel of the mitochondrial preprotein translocase formed by Tim23. Nat. Struct. Biol., (8):1074-1082.

Tzagoloff, A. (1969). Assembly of the mitochondrial membrane system. II. synthesis of the mitochondrial adenosine triphosphatase F1. J. Biol. Chem., (244):5027-5033.

Tzagoloff, A. (1971). Assembly of the mitochondrial membrane system. Role of mitochondrial and cytoplasmic protein synthesis in the biosynthesis of the rutamycin-sensitive adenosine triphosphatase. $J$. Biol. Chem, (246):3050-3056.

Tzagoloff, A. (1995). Ubiquinol-cytochrome c oxidoreductase from S. cerevisiae. Methods Enzymol., (260):51-63.

Tzagoloff, A., Barrientos, A., Neupert, W., and Herrmann, J. M. (2004). Atp10p assists assembly of Atp6p into the Fo unit of the yeast mitochondrial ATPase. J. Biol. Chem, (279(19)):19775-19780.

Valencik, M. L., Kloeckener-Gruissem, B., Poyton, R. O., and McEwen, J. E. (1989). Disruption of the yeast nuclear PET54 gene blocks excision of mitochondrial intron ai5 beta from pre-mRNA for cytochrome $c$ oxidase subunit I. EMBO J., (8):3899-3904.

Vambutas, A., Ackerman, S. H., and Tzagoloff, A. (1991). Purification and characterization of yeast mitochondrial initiation factor 2. Eur. J. Biochem., (201):643-652.

van Bloois, E., Koningstein, G., Bauerschmitt, H., Herrmann, J. M., and Luirink, J. (2007). Saccharomyces cerevisiae Cox18 complements the essential Sec-independent function of Escherichia coli YidC. FEBS J., (274):5704-5713.

van der Laan, M., Bechtluft, P., Kol, S., Nouwen, N., and Driessen, A. (2004). F1Fo ATP synthase subunit $\mathrm{c}$ is a substrate of the novel YidC pathway for membrane protein biogenesis. J. Cell. Biol., (165):213-212.

van der Laan, M., Bohnert, M., Wiedemann, N., and Pfanner, N. (2012). Role of MINOS in mitochondrial membrane architecture and biogenesis. Trends Cell. Biol., (22(4)):185-192.

van der Laan, M., Meinecke, M., Dudek, J., Hutu, D. P., Lind, M., Perschil, I., Guiard, B., Wagner, R., Pfanner, N., and Rehling, P. (2007). Motor-free mitochondrial presequence translocase drives membrane integration of preproteins. Nat. Cell Biol., (9):1152-1159.

Vögtle, F. N., Wortelkamp, S., Zahedi, R. P., Becker, D., Leidhold, C., Gevaert, K., Kellermann, J., Voos, W., Sickmann, A., Pfanner, N., and Meisinger, C. (2009). Global analysis of the mitochondrial N-proteome identifies a processing peptidase critical for protein stability. Cell, (139):428-439.

Volkov, A. N. and van Nuland, N. A. (2012). Electron transfer interactome of cytochrome c. PLoS Comput Biol., 8(12):e1002807.

von der Giezen, M. and Tovar, J. (2005). Degenerate mitochondria. EMBO Rep., (6(6)):525-530.

von Heijne, G. (1986). Why mitochondria need a genome. FEBS Lett., (198):1-4. 
Vonck, J. and Schäfer, E. (2009). Supramolecular organization of protein complexes in the mitochondrial inner membrane. Biochim. Biophys. Acta, (1793):117-124.

Vrbacky, M., Kovalcikova, J., Nuskova, H., Chawengsaksophak, K., Beck, I., Sedlacek, R., Hozak, P., Sedmera, D., and Houstek, J. (2016). TMEM70 gene knockout alters biogenesis of ATP synthase and leads to embryonal lethality in mice. Hum. Mol. Genet., (1837):e21-e22.

Vukotic, M., Oeljeklaus, S., Wiese, S., Vögtle, F. N., Meisinger, C., Meyer, H. E., Zieseniss, A., Katschinski, D. M., Jans, D. C., Jakobs, S., Warscheid, B., Rehling, P., and Deckers, M. (2012). Rcf1 mediates cytochrome oxidase assembly and respirasome formation, revealing heterogeneity of the enzyme complex. Cell Metabol., (15):336-347.

Wagener, N., Ackermann, M., Funes, S., and Neupert, W. (2011). A pathway of protein translocation in mitochondria mediated by the AAA-ATPase Bcs1. Moll. Cell, (44):191-202.

Wagner, K., Gebert, N., Guiard, B., Brandner, K., Truscott, K. N., Wiedemann, N., Pfanner, N., and Rehling, P. (2008). The assembly pathway of the mitochondrial carrier translocase involves four preprotein translocases. Mol. Cell. Biol., (28):4251-4260.

Wagner, K., Perschil, I., Fichter, C. D., and van der Laan, M. (2010). Stepwise assembly of dimeric $\mathrm{F}(1) \mathrm{F}(\mathrm{o})$-ATP synthase in mitochondria involves the small $\mathrm{F}(\mathrm{o})$-subunits $k$ and $i$. Mol. Biol. Cell, (21):1494-1504.

Wai, T. and Langer, T. (2016). Mitochondrial dynamics and metabolic regulation. Trends in Endoc. and Metab., (27(2)):105-117.

Walker, J. E. and Dikson, V. K. (2006). The peripheral stalk of mitochondrial ATP synthase. Biochim. Biophys. Acta, (1757):286-296.

Wang, Z. G. (2000). The assembly factor Atp11p binds to the beta-subunit of the mitochondrial F1ATPase. J. Biol. Chem., (275):5767-5772.

Wang, Z. G., Sheluho, D., Gatti, D. L., and Ackerman, S. H. (2000). The alpha-subunit of the mitochondrial $\mathrm{F}(1)$ ATPase interacts directly with the assembly factor Atp12p. EMBO J., (19):1486-1493.

Wang, Z. G., White, P. S., and Ackerman, S. H. (2001). Atp11p and Atp12p are assembly factors for the F(1)-ATPase in human mitochondria. J. Biol. Chem., (276(33)):30773-30778.

Weber, J. and Senior, A. E. (2003). ATP synthesis driven by proton transport in F1Fo ATP synthase. FEBS Lett., (545):61-70.

Weckbecker, D., Longen, S., Riemer, J., and Herrmann, J. M. (2012). Atp23 biogenesis reveals a chaperonelike folding activity of Mia40 in the IMS of mitochondria. The EMBO J., (31):4348-4358.

Weiss, H., Friedrich, T., Hofhaus, G., and Preis, D. (1991). The respiratory chain NADH dehydrogenase (complex I) of mitochondria. Eur. J. Biochem., (197):563-576.

Wenz, L.-S., Opalinski, L., Schuler, M.-H., Ellenrieder, L., Ieva, R., Böttinger, L., Qiu, J., van der Laan, M., Wiedemann, N., Guirard, B., and et al. (2014). The presequence pathway is involved in protein sorting to the mitochondrial outer membrane. EMBO Rep., (15):678-685.

Wesolowski, M. and Fukuhara, H. (1979). The genetic map of transfer RNA genes of yeast mitochondria: correction and extension. Mol. Gen. Genet, (170):261-275.

Wiedemann, N., Kozjak, V., Chacinska, A., Schönfisch, B., Rospert, S., Ryan, M. T., Pfanner, N., and Meisinger, C. (2003). Machinery for protein sorting and assembly in the mitochondrial outer membrane. Nature, (424):565-571. 
Wiliams, C. C., Jan, C. H., and Weissman, J. S. (2014). Targeting and plasticity of mitochondrial proteins revealed by proximity-specific ribosome profiling. Science, (346):748-751.

Williams, E. H., Bsat, N., Bonnefoy, N., Butler, C. A., and Fox, T. D. (2005). Alteration of a novel dispensable mitochondrial ribosomal small-subunit protein, Rsm28p, allows translation of defective COX2 mRNAs. Eukar. Cell, (4(2)):337-345.

Williams, E. H., Butler, C. A., Bonnefoy, N., and Fox, T. D. (2007). Translation initiation in Saccharomyces cerevisiae mitochondria: functional interactions among mitochondrial ribosomal protein Rsm28p, initiation factor 2, methionyl-tRNA-formyltransferase and novel protein Rmd9p. Cell 4, (175(3)):1117-1126.

Wittig, I. and Schägger, H. (2005). Advantages and limitations of clear-native PAGE. Proteomics, (5):43384346 .

Wittig, I. and Schägger, H. (2008). Structural organization of mitochondria ATP synthase. BBA, (1777):592-598.

Wu, M., Gu, J., Guo, R., Huang, Y., and Yang, M. (2016). Structure of mammalian respiratory supercomplex I1III2IV1. Cell, (167(6)):1598-1609.

Xia, D., Yu, C. A., Kim, H., Xia, J. Z., Kachurin, A. M., Zhang, L., Yu, L., and Deisenhofer, A. (1997). Crystal structure of the cytochrome $b c 1$ complex from bovine heart mitochondria. Science, (277):60-66.

Young, J. C., Hoogenraad, N. J., and Hartl, F. U. (2003). Molecular chaperones Hsp90 and Hsp70 deliver preproteins to the mitochondrial import receptor Tom70. Cell, (112):41-50.

Yun, C. H., Crofts, A. R., and Gennis, R. B. (1991). Assignment of the histidine axial ligands to the cytochrome bH and cytochrome bL components of the bc1 complex from Rhodobacter sphaeroides by site-directed mutagenesis. Biochemistry, (30):6747-6754.

Zara, V., Conte, L., and Trumpower, B. (2007). Identification and characterization of cytochrome bc(1) subcomplexes in mitochondria from yeast with single and double deletions of genes encoding cytochrome bc(1) subunits. FEBS J., (274):4526-4539.

Zeng, X., Barros, M. H., Shulman, T., and Tzagoloff, A. (2008). ATP25, a new nuclear gene of Saccharomyces cerevisiae required for expression and assembly of the Atp9p subunit of mitochondrial ATPase. Mol. Biol. Cell, (19):1366-1377.

Zeng, X., Hourset, A., and Tzagoloff, A. (2007a). The saccharomyces cerevisiae ATP22 gene codes for the mitochondrial ATPase subunit 6-specific translation factor. Genetics, (175(1)):55-63.

Zeng, X., Kucharczyk, R., di Rago, J.-P., and Tzagoloff, A. (2007b). The leader peptide of yeast Atp6p is required for efficient interaction with the Atp9p ring of the mitochondrial ATPase. J. Biol. Chem., (282):36167-36176.

Zeng, X., Neupert, W., and Tzagoloff, A. (2007c). The metalloprotease encoded by ATP23 has a dual function in processing and assembly of subunit 6 of mitochondrial ATPase. Mol. Biol. Cell, (18(2)):617626.

Ziaja, K., Michaelis, G., and Lisowsky, T. (1993). Nuclear control of the messenger RNA expression for mitochondrial ATPase subunit 9 in a new yeast mutant. J. Mol. Biol, (229):909-916.

Zickermann, V., Wirth, C., Nasiri, H., Siegmund, K., Schwalbe, H., Hunte, C., and Brandt, U. (2015). Mechanistic insight from the crystal structure of mitochondrial complex I. Science, (6217):44-49. 


\section{Acknowledgements}

I would like to thank people who were with me during the last couple of years and who contributed so much to my life.

First of all, I would like to thank my supervisor Peter Rehling for giving me the opportunity to work in his lab on such a challenging project. Thank you for providing me with all the necessary tools, including good mood and persistent motivation. You taught me that science is also a little bit of an art.

A big thank you also goes to Blanche Schwappach and Reinhard Lührmann for being members of my thesis committee and Henning Urlaub, Stefan Jakobs and Detlef Doenecke for finding time to be in my extended thesis committee.

I would like to thank my collaborators in Freiburg: Nora Vögtle, for performing protease screens, as well as Marcel Morgenstern and Silke Oeljeklaus, for performing mass spectrometry analyses of my samples.

I am very thankful to Steffen Burkhardt and Kerstin Grüniger, as well as to the other GGNB Office members, for always being so helpful!

My special thanks are devoted to Oleksandr Lytovchenko, who introduced me to science in the lab. I found a great friend in you, Sasha, most probably the best one I have ever had. Although our paths diverged, I am pretty sure that they will cross again many more times in the future.

Silvie Callegary, you helped me so much to improve this thesis. Thank you for your time and patience.

Ridhima Gomkale, thank you for being my source of positive energy!

Isotta Lorenzi, thank you for never giving up on anything and teaching me the same! "Never give up on a membrane", - this phrase of yours was my motto in the lab!

Daryna Tarasenko, thank you for being my soul mate for more than 5 years now. Without you, my PhD would not have been the same.

I am thankful to all present and past members of Rehling's, Meinecke's and Thumm's groups for creating such a great atmosphere in our department and for being very helpful.

Last but not least, I thank Tino Pleiner for being with me in good and bad times. My home is where you are. This means, we will find home anywhere. 